\title{
Advanced Techniques for Reservoir Simulation and Modeling of Nonconventional Wells
}

\section{Final Report}

\author{
Reporting Period Start Date: $\quad$ September 1, 1999 \\ Reporting Period End Date: $\quad$ August 31, 2004 \\ Principal Authors: \\ Louis J. Durlofsky \\ Khalid Aziz \\ Date Report Issued: \\ August 20, 2004 \\ DOE Award Number: \\ DE-AC26-99BC15213 \\ Submitting Organization: \\ Department of Petroleum Engineering \\ School of Earth Sciences \\ Stanford University \\ Stanford, CA 94305-2220
}




\section{Disclaimer}

This report was prepared as an account of work sponsored by an agency of the United States Government. Neither the United States Government nor any agency thereof, nor any of their employees, makes any warranty, express or implied, or assumes any legal liability or responsibility for the accuracy, completeness, or usefulness of any information, apparatus, product, or process disclosed, or represents that its use would not infringe privately owned rights. Reference herein to any specific commercial product, process, or service by trade name, trademark, manufacturer, or otherwise does not necessarily constitute or imply its endorsement, recommendation, or favoring by the United States Government or any agency thereof. The views and opinions of authors expressed herein do not necessarily state or reflect those of the United States Government or any agency thereof. 


\section{Contents}

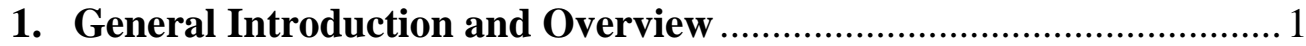

1.1 Advanced Simulation Techniques ................................................ 1

1.2 Calculation of Productivity and Coupling the Well to the Simulator. 3

1.3 Near-Well Upscaling and Optimum Deployment of

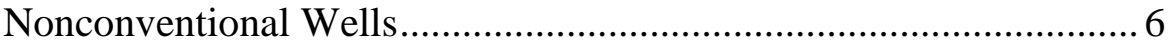

1.4 Variations from the Original Research Plan ...................................9

Part I. Development of Advanced Reservoir Simulation Techniques for Modeling Nonconventional Wells ................................................. 11

2. General Purpose Research Simulator (GPRS)............................. 11

2.1 Overview of GPRS .................................................................... 11

2.2 Linear Solvers in GPRS ............................................................. 13

2.3 Implementation of the Tracer Option ........................................ 42

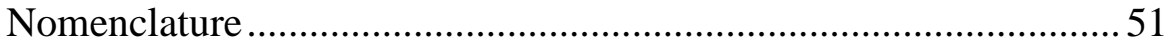

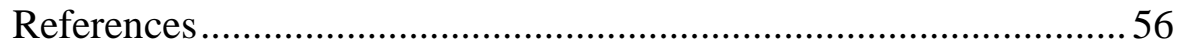

3. Gridding and Upscaling for 3D Unstructured Systems....................60

3.1 Basic Issues with Unstructured Models ........................................ 60

3.2 Overview of the Gridding and Upscaling Procedure ..................... 61

3.3 Grid Generation Methodology ..................................................... 62

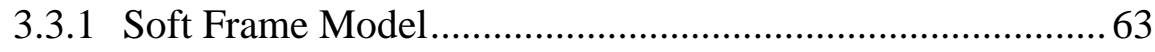

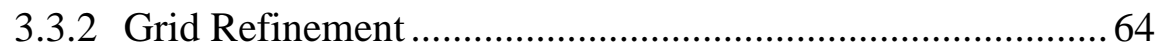

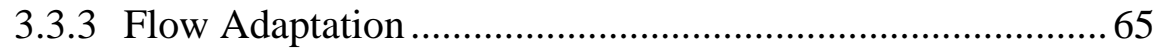

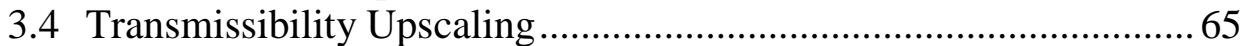

3.5 Streamline Simulation on Unstructured Grids ................................68

3.6 Results for Unstructured Grid Generation and Upscaling............... 70

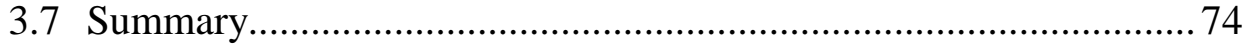

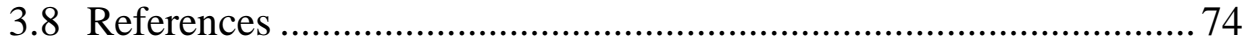

Part II. Coupling of the Reservoir and Nonconventional Wells in Simulators ....................................................................... 76

\section{Numerical Calculation of Productivity Index and Well Index}

for Nonconventional Wells ........................................................... 78

4.1 Semianalytical Formulation ....................................................... 79

4.1.1 Problem Formulation...................................................... 79

4.1.2 Transformation of Anisotropic Reservoir System............... 80

4.1.3 Dimensionless Variables .................................................. 81

4.1.4 Wellbore/Reservoir Coupling Model ................................82

4.1.5 Reservoir Flow ............................................................. 82

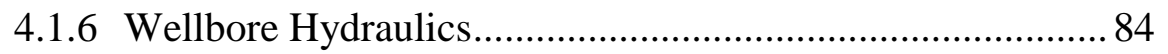

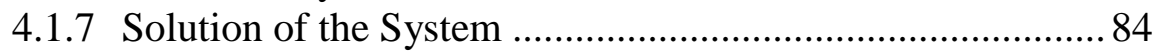

4.1.8 Incorporation of Skin into Solution ..................................... 85 


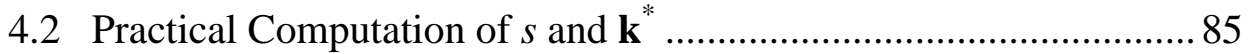

4.2.1 Approaches for the Computation of Global Permeability .... 86

4.2.2 Power Averaging Approach ............................................... 86

4.2.3 Computation of Skin ........................................................... 87

4.2.4 Computation of Permeability and Equivalent Radius in the Altered Zone ................................................................ 88

4.3 Treatment of Downhold Inflow Control Devices .............................8 89

4.4 Modeling Wells with Fixed Bottomhole Pressure ............................ 90

4.5 Example Cases ........................................................................... 92

4.5.1 Offshore Nigeria Reservoir Model .........................................93

4.5.2 Channelized Reservoir Produced with Segmented Well...... 94

4.5.3 Fixed Pressure Well..............................................................97

4.6 Procedure for the Calculation of Well Index .................................. 99

4.7 Calculation of Well Index for a Multiblock Grid ........................... 102

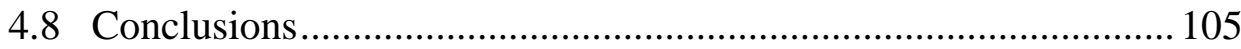

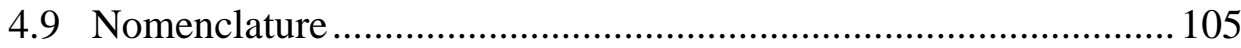

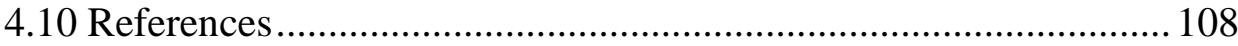

5. Modeling of Multiphase Flow in Wellbores......................................... 111

5.1 Introduction ............................................................................... 113

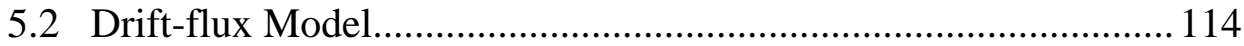

5.2.1 Profile Parameter …………………………………........ 115

5.2.2 Gas-Liquid Drift Velocity................................................. 116

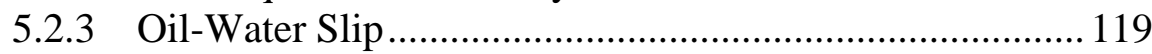

5.2.4 Three-Phase Flow ……………………………............... 120

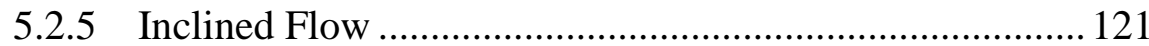

5.3 Experimental Setup and Results ................................................... 121

5.3.1 Experimental Setup .......................................................... 121

5.3.2 Steady State Holdup Results ............................................... 123

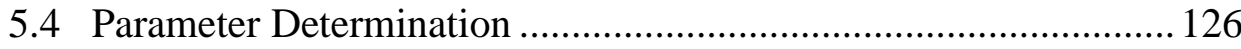

5.5 Results and Discussion for Two-Phase Flows ……………............ 130

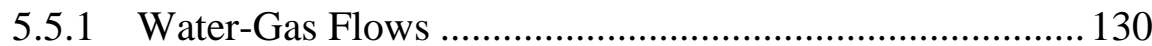

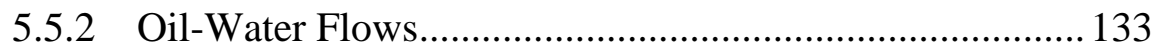

5.6 Three-Phase Parameter Determination ............................................ 136

5.6.1 Application of Water-Gas Results to Three-Phase Flow .. 136

5.6.2 Application of Oil-Water Results to Three-Phase Flow ... 138

5.6.3 Oil-Water Model in Three-Phase Flow.............................. 142

5.6.4 Holdup Prediction from Volumetric Flow Rates ............... 145

5.7 Development of a Multi-Segment Well Simulator - MSWell ...... 146

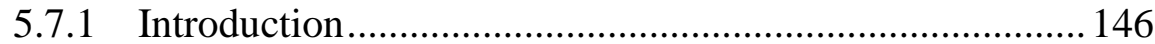

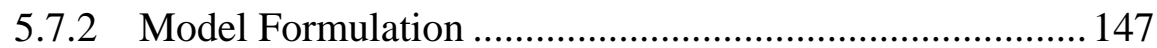

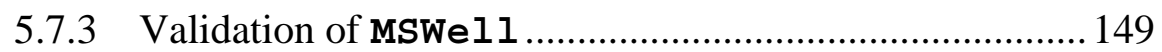

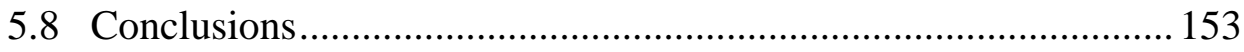

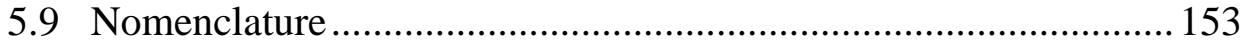

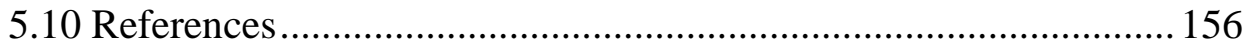


Part III. Novel Approaches to Account for Heterogeneities and the Optimal Deployment of Nonconventional Wells

\section{Accurate Course Scale Simulation of Nonconventional Wells}

in Heterogeneous Reservoirs............................................................ 159

6.1 Well Index and Near-Well Transmissibility Upscaling.................. 162

6.1.1 Local Fine Grid Flow Problem .......................................... 162

6.1.2 Local Coarse Grid Flow Problem ....................................... 167

6.2 Numerical Results for Flow Driven by Horizontal Wells ............... 168

6.2.1 Single Phase Flow Results ................................................. 169

6.2.2 Three-Phase Flow Results.............................................. 174

6.3 Upscaling of Two-Phase Flow Parameters .................................... 176

6.4 Results for Two-Phase Upscaling ................................................ 178

6.5 Upscaling to Radial Grids ............................................................ 183

6.5.1 Multiblock Cross Section Example .................................... 186

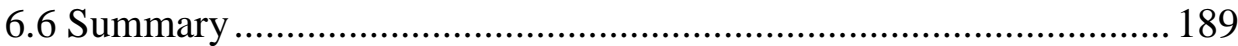

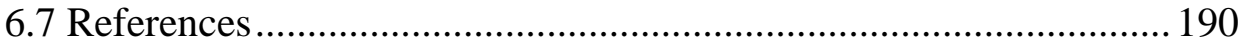

7. Optimization of Nonconventional Well Types and Operations....... 192

7.1 Genetic Algorithm for Optimization of Well Type

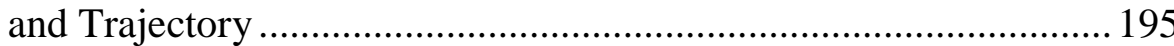

7.2 Optimum Well Determination - Examples .................................... 199

7.2.1 Deterministic Geological Model..................................... 199

7.2.2 Uncertain Geological Model........................................... 202

7.3 Linkage of Smart Well Control and History Matching .................. 204

7.3.1 Optimization Procedure ..................................................... 204

7.3.2 History-Matching Procedure............................................... 204

7.3.3 Overall Optimization and History Matching Procedure ...... 205

7.4 Results Using Optimization and History Matching ........................205

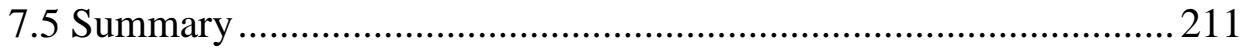

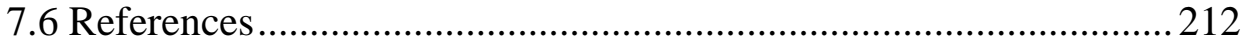




\section{Executive Summary}

Nonconventional wells, which include horizontal, deviated, multilateral and "smart" wells, offer great potential for the efficient management of oil and gas reservoirs. These wells are able to contact larger regions of the reservoir than conventional wells and can also be used to target isolated hydrocarbon accumulations. The use of nonconventional wells instrumented with downhole inflow control devices allows for even greater flexibility in production. Because nonconventional wells can be very expensive to drill, complete and instrument, it is important to be able to optimize their deployment, which requires the accurate prediction of their performance. However, predictions of nonconventional well performance are often inaccurate. This is likely due to inadequacies in some of the reservoir engineering and reservoir simulation tools used to model and optimize nonconventional well performance.

A number of new issues arise in the modeling and optimization of nonconventional wells. For example, the optimal use of downhole inflow control devices has not been addressed for practical problems. In addition, the impact of geological and engineering uncertainty (e.g., valve reliability) has not been previously considered. In order to model and optimize nonconventional wells in different settings, it is essential that the tools be implemented into a general reservoir simulator. This simulator must be sufficiently general and robust and must in addition be linked to a sophisticated well model.

Our research under this five year project addressed all of the key areas indicated above. The overall project was divided into three main categories: (1) advanced reservoir simulation techniques for modeling nonconventional wells; (2) improved techniques for computing well productivity (for use in reservoir engineering calculations) and for coupling the well to the simulator (which includes the accurate calculation of well index and the modeling of multiphase flow in the wellbore); and (3) accurate approaches to account for the effects of reservoir heterogeneity and for the optimization of nonconventional well deployment. An overview of our progress in each of these main areas is as follows.

A general purpose object-oriented research simulator (GPRS) was developed under this project. The GPRS code is managed using modern software management techniques and has been deployed to many companies and research institutions. The simulator includes general black-oil and compositional modeling modules. The formulation is general in that it allows for the selection of a wide variety of primary and secondary variables and accommodates varying degrees of solution implicitness. Specifically, we developed and implemented an IMPSAT procedure (implicit in pressure and saturation, explicit in all other variables) for compositional modeling as well as an adaptive implicit procedure. Both of these capabilities allow for efficiency gains through selective implicitness. The code treats cell connections through a general connection list, which allows it to accommodate both structured and unstructured grids. The GPRS code was written to be easily extendable so new modeling techniques can be readily incorporated. Along these lines, we developed a new dual porosity module compatible with the GPRS framework, as well as a new discrete fracture model applicable for fractured or faulted reservoirs. Both of these methods display substantial advantages over previous implementations. Further, we assessed the performance of different preconditioners in an attempt to improve the efficiency of the linear solver. As a result of this investigation, substantial improvements in solver performance were achieved. 
A set of procedures for the gridding, upscaling and discretization of unstructured simulation models was developed and tested. This work was done in a framework compatible with the GoCad geological modeling software. Unstructured (tetrahedral-based) grids can be conformed to honor complex well geometry, structural information (e.g., faults) and flow information. A streamline simulation technique suitable for unstructured grids was also developed to efficiently assess the quality of the upscaled model relative to the reference geocellular model. Examples demonstrate the performance of these new techniques for unstructured models.

To enable efficient reservoir engineering calculations, we developed a semianalytical solution procedure, based on Green's functions, for the approximate modeling of nonconventional wells. The effects of reservoir heterogeneity, downhole inflow control devices and wellbore pressure losses are included in this formulation. The technique provides efficient estimates for well performance under the assumption of single-phase flow of either oil or gas (as will occur for primary production). Wells can be specified to produce under rate or bottomhole pressure control. The method can be used in conjunction with a simple finite difference calculation to determine the well index for use in general finite difference reservoir simulators. Examples demonstrate that the semianalytical solution technique is able to provide accurate predictions of nonconventional well performance in complex reservoir settings. The techniques developed under this research were implemented into an Advanced Well Modeling (AdWel1) software package.

In addition to the well index, another important aspect of the well model in simulators is the treatment of wellbore flow. Drift-flux models are useful in this regard, as they are able to estimate in situ phase fractions (holdups), which are required for pressure loss calculations. In collaboration with Schlumberger, we collected detailed experimental data for large-diameter (6 inch) pipes that we used to tune drift-flux models for two and three-phase flows. The resulting models provide significantly improved predictions compared to previous models tuned with small-diameter data. A transient wellbore flow simulator was also developed in this work.

Reservoir heterogeneity in the near-well region can have a large impact on well productivity. We developed several techniques to represent subgrid effects in coarse simulation models. These include a near-well upscaling procedure for the determination of an effective well index and well block transmissibilities, the calculation of upscaled (pseudo) relative permeabilities for the nearwell region, and the development of a near-well treatment suitable for use in multiblock simulation models. These techniques provide significant improvements in the accuracy of coarse simulation models relative to standard upscaling methods.

We also developed optimization techniques applicable for nonconventional wells. A genetic algorithm for optimizing well type and placement was developed. The technique incorporates several algorithms to improve computational efficiency. The effects of geological uncertainty were introduced and it was shown that the optimal well depends on the detailed objective function and on the risk attitude of the decision maker. We also developed a technique for optimizing the performance of smart wells. The optimization is achieved through use of a conjugate gradient minimization technique, which is driven by a commercial simulator. We considered the effects of uncertainty in the geological description, as well as engineering uncertainty (e.g., uncertain valve reliability), in the optimization. We also linked the optimization with a history matching technique in order to define a real-time optimization procedure. It was demonstrated that, for the cases considered, the procedure is able to increase production to levels near those obtained when the geology is assumed to be known. 


\begin{abstract}
Research results for a five year project on the development of improved modeling techniques for nonconventional (e.g., horizontal, deviated, multilateral or "smart") wells are presented. The overall program entails the development of enhanced well modeling and general simulation capabilities. The development and features of a new general purpose research simulator (GPRS) are described. The simulator accommodates varying degrees of solution implicitness, handles structured and unstructured grids, and can additionally be used for modeling fractured reservoirs. Advanced linear solution techniques were also implemented. A general gridding and upscaling procedure for unstructured models is presented. The method includes a streamline simulation technique that can be used to assess the accuracy of the coarse model. A semianalytical technique for the modeling of nonconventional wells was developed. This model, implemented into an advanced well modeling software package (AdWell), provides an approximate treatment of reservoir heterogeneity and is able to model downhole inflow control devices and wellbore pressure losses. The well index, which is a key parameter in finite difference well models, can also be computed from AdWell. A drift-flux model for the description of multiphase wellbore flow is presented. Parameters applicable for use in practical systems are determined through tuning to unique experimental data (obtained outside of this DOE sponsored research) on largediameter pipes. The optimum deployment of nonconventional wells is considered next. A genetic algorithm for determining the optimum well type and location is described. A technique for the optimization of smart well control is then presented. The linkage of the method with a historymatching procedure provides a prototype real-time well control strategy.
\end{abstract}




\section{Papers resulting from DOE funded and related research:}

1. Aitokhuehi, I., Durlofsky, L.J., Artus, V., Yeten, B., Aziz, K.: "Optimization of advanced well type and performance," Proceedings of the 9th European Conference on the Mathematics of Oil Recovery, Cannes, France, Aug. 30 - Sept. 2, 2004.

2. Cao, H., Aziz, K.: "Performance of IMPSAT and IMPSAT-AIM models in compositional simulation," SPE paper 77720, presented at the SPE Annual Technical Conference and Exhibition, San Antonio, Sept. 29 - Oct. 2, 2002.

3. Castellini, A., Edwards, M.G., Durlofsky, L.J.: "Flow based modules for grid generation in two and three dimensions," Proceedings of the 7th European Conference on the Mathematics of Oil Recovery, Baveno, Italy, Sept. 5-8, 2000.

4. Chen, Y., Durlofsky, L.J.: "Adaptive local-global upscaling for general flow scenarios in heterogeneous formations" (in review for Transport in Porous Media).

5. Chen, Y., Durlofsky, L.J., Gerritsen, M., Wen, X.H.: "A coupled local-global upscaling approach for simulating flow in highly heterogeneous formations," Advances in Water Resources, 26, 1041-1060, 2003.

6. Chen, Y., Durlofsky, L.J., Wen, X.H.: "Robust coarse scale modeling of flow and transport in heterogeneous reservoirs," to appear in the Proceedings of the 9th European Conference on the Mathematics of Oil Recovery, Cannes, France, Aug. 30 - Sept. 2, 2004.

7. Durlofsky, L.J.: "An approximate model for well productivity in heterogeneous porous media," Mathematical Geology, 32, 421-438, 2000.

8. Durlofsky, L.J.: "Upscaling of geological models for reservoir simulation: issues and approaches," Computational Geosciences, 6, 1-4, 2002.

9. Durlofsky, L.J.: "Upscaling of geocellular models for reservoir flow simulation: a review of recent progress," Proceedings of the $7^{\text {th }}$ International Forum on Reservoir Simulation, Bühl/Baden-Baden, Germany, June 23-27, 2003.

10. Edwards, M.G.: "M-matrix flux splitting for general full tensor discretization operators on structured and unstructured grids," J. Computational Physics, 160, 1-28, 2000.

11. Edwards, M.G.: "Split full tensor discretization operators for structured and unstructured grids in three dimensions," SPE paper 66358, presented at the SPE Reservoir Simulation Symposium, Houston, Feb. 11-14, 2001.

12. He, C., Edwards, M.G., Durlofsky, L.J.: "Numerical calculation of equivalent cell permeability tensors for general quadrilateral control volumes," Computational Geosciences, 6, 29-47, 2002.

13. Karimi-Fard, M.: "Growing region technique applied to grid generation of complex fractured porous media," Proceedings of the 9th European Conference on the Mathematics of Oil Recovery, Cannes, France, Aug. 30 - Sept. 2, 2004. 
14. Karimi-Fard, M., Durlofsky, L.J., Aziz, K.: "An efficient discrete fracture model applicable for general purpose reservoir simulators," SPEJ, 9, 227-236, 2004 (also SPE paper 79699).

15. Mascarenhas, O., Durlofsky, L.J.: "Coarse scale simulation of horizontal wells in heterogeneous reservoirs," Journal of Petroleum Science and Engineering, 25, 135-147, 2000.

16. Prevost, M., Edwards, M.G., Blunt, M.J.: "Streamline tracing on curvilinear structured and unstructured grids," SPEJ, 7, 139-148, 2002 (also SPE paper 66347).

17. Prevost, M., Lepage, F., Durlofsky, L.J., Mallet, J.-L.: "Unstructured 3D gridding and upscaling for coarse modeling of geometrically complex reservoirs," Proceedings of the 9th European Conference on the Mathematics of Oil Recovery, Cannes, France, Aug. 30-Sept. 2, 2004.

18. Sarma, P., Aziz, K.: "Simulation of Naturally Fractured Reservoirs With Dual Porosity Models: Part I-Single-Phase Flow," SPE paper 90231, to be presented at the SPE Annual Technical Conference and Exhibition, Houston, Sept. 26-29, 2004.

19. Shi, H., Holmes, J.A., Diaz, L.R., Durlofsky, L.J., Aziz, K.: "Drift-flux parameters for threephase steady-state flow in wellbores," SPE paper 89836, to be presented at the SPE Annual Technical Conference and Exhibition, Houston, Sept. 26-29, 2004.

20. Shi, H., Holmes, J.A., Durlofsky, L.J., Aziz, K., Diaz, L.R., Alkaya, B., Oddie, G.: “Driftflux modeling of multiphase flow in wellbores," SPE paper 84228, presented at the SPE Annual Technical Conference and Exhibition, Denver, Oct. 5-8, 2003.

21. Valvatne, P.H., Durlofsky, L.J., Aziz, K.: "Semi-analytical modeling of the performance of intelligent well completions," SPE paper 66368, presented at the SPE Reservoir Simulation Symposium, Houston, Feb. 11-14, 2001.

22. Valvatne, P.H., Serve, J., Durlofsky, L.J., Aziz, K.: "Efficient modeling of non-conventional wells with downhole inflow control devices," Journal of Petroleum Science and Engineering, 39, 99-116, 2003.

23. Wen, X.H., Durlofsky, L.J., Edwards, M.G.: "Upscaling of channel systems in two dimensions using flow-based grids," Transport in Porous Media, 51, 343-366, 2003.

24. Wolfsteiner, C., Aziz, K., Durlofsky, L.J.: "Modeling conventional and non-conventional wells," Proceedings of the $6^{\text {th }}$ International Forum on Reservoir Simulation, Hof/Salzburg, Austria, Sept. 3-7, 2001 (also appearing in the Proceedings of the International Energy Agency Symposium on Enhanced Oil Recovery, Vienna, Austria, Sept. 9-12, 2001).

25. Wolfsteiner, C., Durlofsky, L.J.: "Near-well radial upscaling for the accurate modeling of nonconventional wells," SPE paper 76779, presented at the SPE Western Regional Meeting, Anchorage, May 20-22, 2002.

26. Wolfsteiner, C., Durlofsky, L.J., Aziz, K.: "Calculation of well index for non-conventional wells on arbitrary grids," Computational Geosciences, 7, 61-82, 2003. 
27. Wolfsteiner, C., Durlofsky, L.J., Aziz, K.: "Approximate model for productivity of nonconventional wells in heterogeneous reservoirs, SPEJ, 5, 218-226, 2000 (also SPE paper 56754).

28. Wolfsteiner, C., Durlofsky, L.J. and Aziz, K.: "Efficient estimation of the effects of wellbore hydraulics and reservoir heterogeneity on the productivity of non-conventional wells," SPE paper 59399, presented at the SPE Asia Pacific Conference, Yokohama, Japan, April 25-26, 2000.

29. Yeten, B., Brouwer, D.R., Durlofsky, L.J., Aziz, K.: "Deployment of smart wells in the presence of geological and engineering uncertainty" (to appear in Journal of Petroleum Science and Engineering).

30. Yeten, B., Durlofsky, L.J., Aziz, K.: “Optimization of smart well control,” SPE paper 79031, presented at the SPE International Thermal Operations and Heavy Oil Symposium and International Horizontal Well Technology Conference, Calgary, Nov. 4 - 7, 2002.

31. Yeten, B., Durlofsky, L.J., Aziz, K.: “Optimization of intelligent well control,” World Oil, 224, 35-40, March 2003.

32. Yeten, B., Durlofsky, L.J., Aziz, K.: “Optimization of nonconventional well type, location and trajectory," SPEJ, 8, 200-210, 2003 (also SPE paper 77565).

33. Yeten, B., Wolfsteiner, C., Durlofsky, L.J., Aziz, K.: "Approximate finite difference modeling of the performance of horizontal wells in heterogeneous reservoirs," SPE paper 62555, presented at the SPE Western Regional Meeting, Long Beach, June 19-23, 2000. 


\section{General Introduction and Overview}

Reservoir simulation represents an essential tool for the management of oil and gas reservoirs. A key aspect of reservoir simulation is the representation of the well in the simulator and the linkage of the well to the reservoir. These issues are particularly important in the modeling of advanced or nonconventional wells, which include horizontal, highly deviated, multilateral and "smart" or "intelligent" wells with downhole sensors and inflow control devices. Accurate tools for modeling and optimizing advanced wells are needed, as the costs associated with these wells are very high. However, existing techniques are often inadequate for the modeling of nonconventional wells, as these wells are significantly more complex than simple vertical or slightly deviated wells. Further, the opportunity for the real-time optimization of smart well performance introduces additional challenges.

This overall project was directed toward significantly improving the modeling of nonconventional wells along the lines indicated above. Our research was divided into three main areas. These include the development of (1) advanced reservoir simulation techniques for modeling nonconventional wells; (2) improved techniques for computing well productivity (for use in reservoir engineering calculations) and for coupling the well to the simulator (which includes the accurate calculation of well index and the modeling of multiphase flow in the wellbore); and (3) accurate approaches to account for heterogeneity in the near-well region and for the optimization of nonconventional well deployment. We now provide an overview of our work over the course of this five year project, much of which was presented in previous Annual Reports to DOE.

\subsection{Advanced Simulation Techniques}

Nonconventional well modeling represents one component of the overall reservoir simulation. Because our research targets the development of an improved overall simulation capability, we have focussed considerable effort toward the development of a general purpose object-oriented research simulator. This simulator accommodates general grids and a variety of procedures for treating the system variables. The simulator, referred to as GPRS (General Purpose Research Simulator), is written to be easily extendable so new modeling techniques can be readily incorporated by future researchers. GPRS contains a general compositional formulation and allows for the use of different sets of primary and secondary variables and varying degrees of implicitness, including an adaptive implicit capability. 
We demonstrated that the use of a newly developed IMPSAT procedure (implicit in pressure and saturation, explicit in composition) can provide highly efficient models for compositional simulations. IMPSAT contains a degree of implicitness intermediate between IMPEC (implicit in pressure, explicit in component mass fractions) and fully implicit. GPRS also allows one or more of the component mass fractions to be treated implicitly, which provides for great flexibility in the solution procedure. The adaptive implicit capability allows for the fully implicit treatment of a limited number of cells (with the rest handled using IMPEC or IMPSAT). This will enable highly efficient simulations and, eventually, parallel scalability, as it is often a very small percentage of grid blocks that require a fully implicit treatment. Details regarding GPRS development were described in our Year 2 and 3 Annual Reports.

GPRS is actively maintained and supported in-house by an experienced software engineer. Enhancements are systematically tested and documented. GPRS is a key software application within our research group and it is widely used as a vehicle for new research developments. Many companies and external research institutions have also requested and actively use GPRS for prototyping new simulation capabilities.

Following the development of the basic simulator, several new capabilities were developed for GPRS. These include a new dual porosity formulation (though this is not yet part of the production version) for the modeling of naturally fractured reservoirs and sophisticated linear equation solution procedures (which have been implemented in the production version). The dual porosity model incorporates (as a preprocessing step) a general numerical procedure to calculate the shape factor for arbitrarily shaped fractures and matrix blocks. Unlike previous models, this dual porosity description accounts for transient pressure effects in the matrix-fracture transfer function. An approximate representation of two-phase flow effects on the matrix-fracture transfer was also included. This model was described in detail in the Year 4 Annual Report.

This year our emphasis was on the development of efficient linear solution techniques. Toward this end, we investigated in detail the properties of the pressure matrix and the performance of three different preconditioners in an attempt to improve the efficiency of linear solver. As a result of this investigation, very significant improvements in solver performance were achieved. The current solver implementation is limited to serial (rather than parallel) computations, though the solver formulation is appropriate for extension to parallel calculations. It is also well suited to 
handling fully unstructured simulation models. A general overview of GPRS, as well as a description of our linear solver developments, appears in Chapter 2 of this report.

Because GPRS is based on a general connection-list representation of the grid system, unstructured models can be readily accommodated. We have developed two new capabilities based on unstructured grid representations. The first such capability, a discrete fracture model (described in the Year 4 Annual Report), compliments the dual porosity formulation noted above. This discrete fracture model incorporates the so-called "star-delta" transform (originally developed within the context of a network of resistors) to treat flow at fracture intersections. This acts to modify the connections such that the fracture intersection need not be resolved explicitly, which results in significantly more efficient simulations. The discrete fracture model was applied to two and three-dimensional example cases including a model of a damage zone around a strikeslip fault.

Unstructured grids are well suited for the modeling of complex geological features and the interaction of nonconventional wells with the reservoir. The use of such grids in simulators requires the development of grid generation, upscaling, and simulation techniques compatible with unstructured representations. In Chapter 3, we describe our work in these areas. We interacted closely with researchers in the GoCad group (based in Nancy, France) on the grid generation capabilities and our implementation uses GoCad gridding technology. Our approach allows grids to honor both geological and well features, in addition to being adapted to the flow field. Upscaling and streamline simulation techniques allow for the generation and evaluation of the resulting grids. Examples demonstrate the capabilities of our new gridding and upscaling techniques for unstructured models. We note that gridding and discretization issues were also addressed in the Year 1 and 2 Annual Reports.

\subsection{Calculation of Productivity and Coupling the Well to the Simulator}

An important component of nonconventional well modeling is the linkage between the well and the simulator. This issue is closely related to the representation of nonconventional well performance via (standalone) semianalytical solution techniques. Such techniques are generally based on a Green's function representation, in which the well, rather than the reservoir, is discretized. These methods are generally much less demanding computationally than finite difference 
simulation. In addition, they have no difficulty modeling wells that exhibit geometric complexity (e.g., multilaterals) since the reservoir is not gridded.

Under this project, we have developed several advanced capabilities for semianalytical well modeling (these developments are described in each of the four Annual Reports). These developments have been implemented into an object-oriented Advanced Well Modeling Capability called AdWell. The formulations are applicable for the single-phase flow of either oil or gas, so they are most appropriate for modeling primary production. Previous semianalytical techniques are restricted to homogeneous or layered reservoirs. However, reservoir heterogeneity can significantly impact nonconventional well performance, so it is important that these effects be included in the model. We developed a new heterogeneity representation that is compatible with our semianalytical modeling procedure. This representation is referred to as the $s-\mathbf{k}^{*}$ permeability model because permeability is represented in terms of an average background permeability $\mathbf{k}^{*}$ and a near-well effective skin $s$ that varies along the trajectory of the well. The effective skin captures the effects of near-well permeability variation. The $s-\mathbf{k}^{*}$ model in AdWell is quite general and allows for the approximation of the effects of varying fine scale permeability anisotropy and bimodal permeability distributions. Its application to realistic reservoir models was demonstrated in the Year 4 Annual Report.

Several other important enhancements to the semianalytical model were also introduced into AdWell. These include the modeling of downhole inflow control devices and the ability to prescribe rate or bottomhole pressure (BHP) well conditions. Downhole valves (described in the Year 2 Annual Report) are modeled through the use of a multisegment well representation, where some segments represent valves. This capability was applied to model both single heterogeneous reservoirs as well as multiple reservoirs (at different pressures) produced with a single well. The Green's function used in our formulation is directly applicable for use with fixed rate wells. The specification of BHP required us to introduce a time stepping procedure (as well rate now varies in time). This capability, in addition to the ability to specify multiple wells with time varying constraints, was also implemented into AdWell (as described in detail in the Year 3 Annual Report).

The semianalytical well model can also be used to determine the well index for use in a finite difference simulator. The well index, which is a key parameter in the simulation well model, re- 
lates the local wellbore pressure to the well block pressure. The semianalytical model provides the wellbore pressures and well flow rates. These can be used in a preliminary finite difference calculation in which well flow rates are specified in each grid block intersected by the well. The resulting grid block pressures are then used in conjunction with the semianalytical wellbore pressures and well rates to determine the well index. We developed and implemented into AdWell a very general procedure along these lines (described in detail in the Year 3 Annual Report). Our implementation is applicable for arbitrary well geometries on general (e.g., unstructured) grids. The method can account approximately for subgrid heterogeneity via the $s$-k ${ }^{*}$ permeability model. Examples with multilateral wells and complex grids demonstrated the capabilities of the technique as well as the potential errors inherent in simple (default) procedures.

Chapter 4 of this report describes and illustrates our semianalytical well modeling capabilities. The examples illustrate the range of problems that can be addressed accurately and efficiently through use of the AdWell software package.

In some cases, wellbore hydraulics can impact the performance of nonconventional wells. Such effects are most important when drawdown is comparable to the pressure loss along the wellbore. In order to model these effects, it is important to predict with reasonable accuracy the in situ phase fractions (i.e., phase holdups) within the wellbore. This may be difficult, however, as the degree of slip between the various phases is a complex function of flow rates, well inclination and phase properties.

Drift-flux models are well suited for modeling slip (and thus phase holdups) in wellbore or pipe flows. These models contain many parameters, however, which must be determined from experimental data. In a project outside of this DOE funded work, we collaborated with Sclumberger Cambridge Research on an experimental program to measure phase holdups in large diameter (6 inch) pipe. Two-phase oil-water and water-gas flows and three-phase oil-water-gas flows were studied at inclinations between vertical and horizontal for a variety of flow rate combinations. We then used this data in conjunction with an optimization procedure for the determination of parameters for the drift-flux model. The level of agreement between the experimental data and the predictions of the drift-flux model is reasonably good in most cases and demonstrates a considerable improvement over that provided by existing parameters (determined from experiments with small diameter pipe). 
As a first step toward the incorporation of our drift-flux model into the general well model in GPRS, we developed a standalone three-phase transient wellbore flow model. This model provides phase holdups and pressure along the wellbore as a function of time. In future work, we plan to implement a full multisegment well model that includes this drift-flux representation into GPRS. The drift-flux model and parameter optimization and the three-phase transient flow model are described in detail in Chapter 5.

\subsection{Near-Well Upscaling and Optimum Deployment of Nonconventional Wells}

Geostatistical modeling approaches generally provide finely gridded geological characterizations, though these models are often too detailed to be used directly for reservoir simulation. Some type of averaging, or upscaling, is therefore required. Many such approaches have been developed and existing methods are capable of providing accurate coarse scale reservoir descriptions in regions away from wells. In the near-well region, however, standard upscaling approaches can yield significant inaccuracies and improved coarse scale descriptions are necessary. This is the case for both single-phase flow parameters (e.g., upscaled permeability or transmissibility) as well as two-phase parameters (upscaled relative permeability or pseudorelative permeability).

In this work we developed several methods capable of providing coarse scale finite difference models of nonconventional wells. We implemented a general approach for the determination of coarse scale well index and well block transmissibility (described in detail in the Year 1 Annual Report). This method entails the solution of a local steady-state single-phase near-well problem (comprising the fine scale region corresponding to the well block and one or two rings of neighboring blocks). A post-processing (averaging) of the pressure and flow rates determined from this solution provides an estimate of the upscaled well index and well block transmissibilities. We demonstrated that improved accuracy can be achieved if we "optimize" the coarse scale parameters to attain closer agreement between the fine and coarse scale local problems. The im-

provement in accuracy in the coarse model over that achieved using standard methods was shown to be substantial in many cases.

This single-phase parameter upscaling approach described above was extended in two important directions. In Chapter 6 of this report, we describe our work on the development of upscaled relative permeabilities in the near-well region. This method is based on an optimization 
(along the lines indicated above) for a local two-phase flow problem. By forcing agreement between the averaged phase flow rates, we determine upscaled relative permeabilities. We demonstrate that this can provide improved coarse scale descriptions, particularly in the case of injection wells.

We also developed a single-phase parameter upscaling procedure for radial models within the context of multiblock simulation models (discussed in detail in the Year 3 Annual Report). Such models are structured locally but unstructured globally, so they are capable of resolving complex geometric features. We developed a "hole" well model, in which the nonconventional well is represented as a "tunnel" through the simulation grid with a near-radial grid around the well trajectory. In order to avoid resolving down to the scale of the wellbore, which would be extremely costly, we developed an upscaling technique to account for the unresolved heterogeneity in the near-well region. This enables the use of a larger diameter "hole" and leads, as a result, to computational savings. The near-well upscaling work discussed here is described in Chapter 6.

The optimization of nonconventional well deployment is a challenging and important problem. In this work, we addressed several aspects of this broad optimization problem. We first considered the optimization of nonconventional well type, location and trajectory. This problem is more complicated than conventional well optimization problems because of the wide variety of possible well types (i.e., number, location and orientation of laterals) that must be considered. The optimization procedure entails a genetic algorithm applied in conjunction with several acceleration routines that include an artificial neural network, a hill climber, and a near-well upscaling technique. The form of the "chromosome" (i.e., string of bits that defines possible solutions within the context of the genetic algorithm) allowed the well type to "evolve" over the course of the optimization.

The overall methodology was applied to a number of problems involving different reservoir types and fluid systems. We showed that the objective function (cumulative oil produced or net present value of the project) always increased relative to its value in the first generation of the optimization, in some cases by $30 \%$ or more. The effects of uncertainty in the reservoir description on the optimization were also explored. We demonstrated that the optimal well depends on the risk attitude of the decision maker. This work was discussed in detail in the Year 3 Annual Report and is described here in Chapter 7. 
Smart wells contain downhole sensors and inflow control devices. Although smart wells hold great promise, operating them in an optimal fashion remains a challenge. We completed several projects addressing issues related to the optimum deployment of smart wells. For the optimization of smart well control, we introduced a conjugate gradient optimization technique that uses numerical gradients provided by a commercial reservoir simulator. The procedure is computationally intensive, but it was shown to provide downhole valve settings that can result in significantly improved recoveries (e.g., as high as $65 \%$ over the uninstrumented base case).

Decisions regarding the deployment of smart wells must account for the effects of uncertain geology and valve reliability. Geostatistical techniques can be employed to generate multiple (plausible) geological scenarios. Statistical models for downhole valve reliability were not, however, applied previously within this context. For this purpose, we introduced a valve failure model based on the Weibull lifetime distribution. This model accounts for failures occurring with different probabilities over the life of each downhole valve. A decision analysis procedure was presented to enable decision making under geological and engineering uncertainty for decision makers with varying risk attitudes. Although uncertainty in valve reliability decreased the benefit of smart wells, it was shown that they are still the preferred choice in many settings. This smart well optimization work was described in detail in the Year 4 Annual Report.

Our recent work on smart well optimization, described in Chapter 7, entails the linkage of a history matching procedure with the well optimization algorithm to accomplish a prototype realtime optimization strategy. The method allows for the continuous updating of the geological model using data from downhole sensors. Downhole sensor data, generated by simulating production from one particular geostatistical realization, are assumed to provide inflow rates for each fluid phase in each instrumented branch of a multilateral well. We show that the combined procedure is capable of increasing cumulative production significantly, to levels very near those obtained when the geology is assumed to be known. Because history-matched models are nonunique, there is potential benefit in optimizing valve settings over multiple history-matched models. We investigate this issue and demonstrate that the use of multiple history-matched models does provide significant improvement over optimization using just a single history-matched model in some cases. 


\subsection{Variations from the Original Research Plan}

Taken in total, our accomplishments on this research are quite close to the research proposed in our original DOE proposal. There are, however, some areas we explored much more broadly and others we put less effort into than was originally anticipated. We now highlight these issues.

Under Task 1, we put less effort into parallelization of GPRS than we had anticipated. This was because we spent more effort on GPRS development and on the implementation of unstructured gridding and upscaling techniques than we had planned. Having developed these techniques, and having implemented state of the art sequential linear solution techniques, we are now ready to begin actively parallelizing GPRS. It is important to recognize that many of our GPRS developments, such as the adaptive implicit procedure, enable parallelization, so much of our work is indeed applicable toward this goal. In addition, we did progress on the parallelization of simulation code outside of GPRS. Specifically, we successfully parallelized and tested a coupled geomechanics - fluid flow simulator (as described in the Year 4 Annual Report). Our lack of progress on the parallelization of GPRS is more than offset, we feel, by our extensive progress on other features of the code as well as by our efforts on coding and maintenance standardization.

Under Task 2, we did not complete the implementation of the multisegment well model into GPRS. This implementation is a more significant task than we realized. We did, however, proceed much further than we had anticipated in the development of drift-flux models for two and three-phase flows. A high priority in our future work will be to implement the multisegment well model, containing our drift-flux models, into GPRS. We note also that our progress on semianalytical well modeling (and the determination of well index) significantly exceeded our original plan. For example, the development and dissemination of the AdWell code was not a component of the original plan.

Our progress on Task 3 was extensive, but it should be noted that we slightly modified the original plan shortly after the start of the work. In our original proposal, we had planned to implement a simulation while drilling capability. We soon realized, however, that this would require that we address issues of drilling, data collection, log interpretation, etc., which were outside of our areas of interest. Therefore, we modified the subtask to emphasize the development of tools for use with simulation while drilling. These tools include fast simulation capabilities (e.g., semianalytical modeling including heterogeneity, near-well upscaling) and optimization. 
We progressed much further in our optimization work than we had anticipated. For example, our original proposal did not include the optimization of smart wells, an area in which we are pleased to report significant progress. 


\title{
Part I. Development of Advanced Reservoir Simulation Techniques for Modeling Nonconventional Wells
}

Our main effort under this task involved the development of a general purpose research simulator (GPRS), described in Chapter 2. Aspects of this simulator were presented in detail in previous Annual Reports. The initial development work for GPRS was performed by Hui Cao in his PhD work. Subsequent contributors are Yuanlin Jiang (MS student, implemented new linear solvers and a tracer simulation option), Pallav Sarma (MS student, developed a dual-porosity model compatible with GPRS), Dr. Huanquan Pan (software engineer responsible for code management) and Prof. Hamdi Tchelepi (who is now directing GPRS development). A number of other students in our group have also contributed code or performed testing on GPRS. This simulator will be used in the future as a platform for research in a variety of areas, including general formulations, advanced well modeling, gridding, discretization and parallelization.

In Chapter 3, a general gridding and upscaling procedure applicable for unstructured models is described. A streamline simulation technique designed for unstructured models is also presented. These contributions represent the recent $\mathrm{PhD}$ work of Mathieu Prevost.

\section{General Purpose Research Simulator (GPRS)}

\author{
By Yuanlin Jiang, Hamdi Tchelepi and Khalid Aziz
}

\subsection{Overview of GPRS}

Reservoir simulation is a critical tool for the optimum development of oil and gas fields. While reservoir simulators of various degrees of sophistication have been in use for over 40 years, they have become essential tools for reservoir management over the last quarter century. The industry continues to demand more robust, faster reservoir simulators that provide wide, and ever-growing, functionality.

Several major oil companies and service companies have been developing new simulators to meet future needs. However, the underlying fundamental research needed to meet the 
challenge of expanding numerical reservoir simulation capabilities is largely done at universities like Stanford. The use of simulators for optimization of field development and operation under geological uncertainty is becoming possible with current hardware and software technologies. This places new demands on future simulators in terms of speed and robustness.

Reservoir simulation research at a University like Stanford requires a versatile framework for developing and maintaining a modular General Purpose Research Simulator (GPRS). In a research simulator of this type, modularity and ease of maintenance are more important than speed. A simulator like this allows researchers to explore different numerical techniques for modeling complex oil recovery processes without undue need for programming from scratch. Such a GPRS has been developed at Stanford University. It is evolving into a major research tool in the field of reservoir simulation.

The major focus of our research is the development of a compositional simulator that can also handle black oil problems in an efficient manner with the flexibility to explore new approaches. The simulator design allows for complete flexibility in the type of grid used, which is a significant advantage. As reported earlier the simulator has already been extended to handle dual porosity/dual permeability systems. The detailed design of the simulator is discussed in our previous reports and in the thesis of Cao (2002).

In this report we discuss our recent work on linear solvers in GPRS and the implementation of a tracer option. We have also improved the handling of phase behavior in GPRS.

Plans are now underway to add modules to handle:

- thermal processes,

- geomechanics,

- surface facilities,

- advanced wells,

- automated history matching, and

- optimization.

The primary goal of our work is the exploration of new, more efficient numerical techniques, not the development of code for direct commercial applications. This flexible 
simulation framework is used extensively by researchers at Stanford. Moreover, several oil producing and service companies use our code for their in-house research.

Our investigations have resulted in the development of a new compositional model that promises to be far more efficient than existing models. In the new IMPSAT (IMplicit Pressure and SATurations) model, only the pressure and saturations are treated implicitly, regardless of the number of components in the system. In conventional compositional simulations either just pressure is treated implicitly (IMPES or IMPEC) or all the primary variables are treated implicitly (Fully Implicit Model or FIM). In the first approach the timestep is highly restricted, and in the second approach the amount of work per timestep increases sharply as the number of components needed to describe the system increases. We have shown that IMPSAT is significantly more stable than conventional IMPES models, and that one can usually take timestep sizes comparable to those obtained with the FIM approach.

Our General Purpose Research Simulator (GPRS) uses a general compositional formulation that allows any level of implicitness. All of the operations are performed on individual gridblocks, so that different implicit levels can be used for different gridblocks (Adaptive Implicit Method, AIM).

In a reservoir simulator, a significant part of the computational effort is spent on the solution of the linear systems of equations. We have started a major effort on the development of linear solvers for large-scale problems. This work is discussed in the next section. Our work on tracer modeling is presented in Section 2.3.

\subsection{Linear Solvers in GPRS}

In reservoir simulation, discretization of the governing equations leads to a system of nonlinear algebraic equations. Solution of the nonlinear system is usually obtained using Newton's method. Each Newton iteration requires the solution of a linear system of equations. The size of the linear system depends on the number of gridblocks, or cells, in the model and the number of implicit unknowns per gridblock. In the IMPES method (Garder and Stone, 1961; Coats, 2000), pressure is the only unknown in a gridblock. The linear system that describes the pressure in the IMPES formulation is usually diagonally dominant and nearly symmetric. The linear system associated with the fully implicit method (FIM) has multiple unknowns per gridblock and is not necessarily diagonally dominant. Furthermore, it is generally highly nonsymmetric (Wallis, 1983). 
In the course of a simulation run, hundreds or even thousands of timesteps are required. For each timestep, several Newton iterations are usually needed to solve the nonlinear system. Repeated solution of the linear system in the course of a simulation is usually the most CPU time intensive activity. As a result, the overall performance of a reservoir simulator depends on having a robust and computationally efficient linear solver.

The solution of the linear system involves a preconditioning step. The performance of a particular preconditioning method depends on the nature of the target linear system. The reservoir simulation equations are particularly challenging to deal with because they posses a mixed character. Flow (e.g., pressure and total velocity) is governed by (near) elliptic equations where global coupling between the unknowns is important, while transport related quantities display (near) hyperbolic character, where gradients may be steep but highly localized.

Several preconditioning methods have been used for reservoir simulation. Examples include Incomplete LU decomposition (ILU), Successive Over Relaxation (SOR), Gauss-Siedel (GS). More recently, multigrid methods have been used. Our objective here is to improve the robustness and performance of GPRS, especially for large-scale heterogeneous problems with coupled wells, which have multi-block completions. We focus on a two-stage preconditioning strategy that is tuned to the mixed character of the linear systems encountered with fully implicit simulation. Specifically, we concentrate on the Constrained Pressure Residual (CPR) method developed by Wallis (1983). The effectiveness of CPR for FIM linear systems on structured grids is well established (Wallis, 1983, 1985). Here, we describe the components of a CPR preconditioning strategy particularly suited for fully implicit simulation of large-scale heterogeneous reservoir models discretized using unstructured grids.

\section{Formulations and Solvers in GPRS}

GPRS is a general-purpose, unstructured-grid, reservoir simulator that offers the following formulations:

- IMPES: Implicit pressure, explicit saturations and component mole fractions (Stone and Garder, 1961; Coats, 2000). 
- IMPSAT: Implicit pressure and saturations, explicit component mole fractions (Cao and Aziz, 2002).

- FIM: Fully implicit method (Aziz and Settari, 1979)

- AIM: Adaptive implicit method (Thomas and Thurnau, 1983; Forsyth and Sammon, 1986) with three levels of implicitness.

GPRS is the first (and only) general-purpose reservoir simulator that employs a general AIM formulation with three levels of implicitness, namely, FIM, IMPSAT and IMPES. The development of special algorithms that deal effectively with AIM systems for unstructured grid is ongoing. Here we focus on scalable (i.e., efficient for large problems) linear solution methods for IMPES and fully implicit models.

GPRS provides several well control methods, including BHP (Bottom Hole Pressure) control and rate controls, namely, water, oil, liquid, and gas rate, as shown in Fig 2-1.

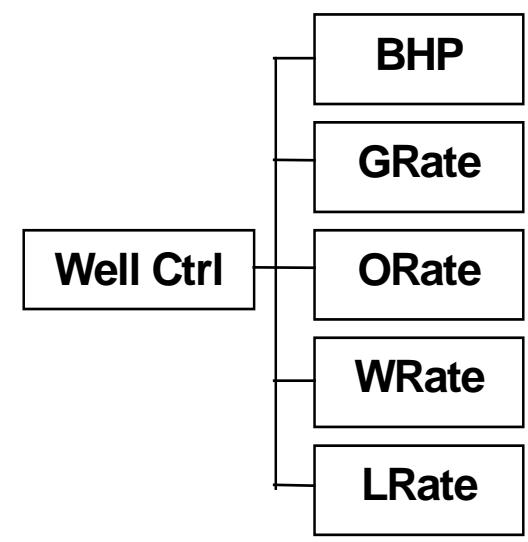

Fig. 2-1: Available well controls in GPRS

\section{Linear Solvers}

Several solvers are available in GPRS:

- Solver 1: Full matrix direct solver from Linear Algebra Package (LAPACK) (Anderson, 1999)

- Solver 2: Banded matrix direct solver from LAPACK (Anderson, 1999)

- Solver 3: BlitzPak iterative solver (Landmark Graphics Corporation, 1998) 
- Solver 4: Generalized Minimum Residual iterative solver (GMRES) (Saad and Schultz, 1986) from the Iterative Math Library (IML) (Dongarra et al., 1996)

For large problems, iterative solvers are memory efficient and much faster than direct solvers. Preconditioners are used to accelerate the convergence rate of iterative methods. Even for relatively simple cases, solvers may not converge without a high-quality preconditioner. The available preconditioners in GPRS are:

- Pre1: Diagonal scaling

- Pre2: Block diagonal scaling

- Pre3: Incomplete LU factorization (ILU)

- Pre4: True-IMPES CPR (AMG + ILU)

- Pre5: Quasi-IMPES CPR (AMG + ILU)

- Pre6: AMG (Algebraic MultiGrid) for IMPES only.

- Pre7: True-IMPES CPR (SAMG + ILU)

- Pre8: Quasi-IMPES CPR (SAMG + ILU)

- Pre9: SAMG (for IMPES only)

\section{Constrained Pressure Residual (CPR) Method}

While there are several preconditioning options in GPRS, the most successful ones are those based on the two-stage CPR approach (Pre7 and Pre8), where we combine a multigrid solver with conventional methods to yield a highly effective algorithm for large heterogeneous FIM problems. The general form of a sequential two-stage preconditioner is:

$$
M_{1,2}^{-1}=M_{2}^{-1}\left[I-A M_{1}^{-1}\right]+M_{1}^{-1}
$$

where $M_{1}$ and $M_{2}$ are the preconditioning matrices for the first and second stages, respectively. The first stage is an approximate solution of a global system that resembles the pressure equation of the IMPES formulation. The second stage preconditioner is a local operator applied to the fully implicit matrix $A$. The CPR method has proved to be robust and efficient for FIM problems on structured grids (Wallis, 1983, 1985). 
The FIM system of linear equations can be written as:

$$
A \vec{u}=\vec{r},
$$

where $A$ is the coefficient matrix, $\vec{u}$ is the vector of unknowns, and $\vec{r}$ is the residual. We permute the above system by ordering the pressure equations and unknowns last and ordering all the other equations and unknowns first. This yields

$$
\left[\begin{array}{ll}
A_{S S} & A_{S P} \\
A_{P S} & A_{P P}
\end{array}\right]\left[\begin{array}{l}
u_{S} \\
u_{P}
\end{array}\right]=\left[\begin{array}{l}
r_{S} \\
r_{P}
\end{array}\right]
$$

where subscript $P$ refers to pressure and subscript $S$ to the remaining unknowns. The first step in CPR is to apply an IMPES-like reduction to Eq. 2-3. Both sides of Eq. 2-3 are multiplied by a matrix of the form

$$
N=\left[\begin{array}{ll}
I & 0 \\
Q & I
\end{array}\right],
$$

to obtain

$$
\left[\begin{array}{cc}
A_{S S} & A_{S P} \\
\hat{A}_{P S} & \hat{A}_{P P}
\end{array}\right] \quad\left[\begin{array}{l}
u_{S} \\
u_{P}
\end{array}\right]=\left[\begin{array}{l}
r_{S} \\
\hat{r}_{P}
\end{array}\right] .
$$

Now the pressure matrix is extracted as follows

$$
A_{p}=C^{T} N A C
$$

where

$$
C^{T}=\left[\begin{array}{ll}
0 & I
\end{array}\right]
$$

The effectiveness of the CPR method depends, to a large extent, on the quality of the pressure matrix, $A_{p}$. We use two approaches for the IMPES-like reduction step that produces $A_{p}$, namely, Quasi-IMPES and True-IMPES. For the Quasi-IMPES scheme, we ignore the off-diagonal $A_{P S}$ terms and eliminate the diagonal $A_{P S}$ part using the inverse of the 
block-diagonal of $A$. A similar approach is used for the True-IMPES scheme, except that we work with a Jacobian matrix consistent with the IMPES formulation.

The first-stage preconditioner can be expressed as

$$
M_{1}^{-1}=C A_{p}^{-1} C^{T} N
$$

The second-stage preconditioner, $M_{2}^{-1}$, is a local operator that is applied to the entire system. Finally, the full form of the CPR operator is

$$
M_{C P R}^{-1}=M_{2}^{-1}\left[I-A C A_{p}^{-1} C^{T} N\right]+C A_{p}^{-1} C^{T} N
$$

GMRES with AMG preconditioning is used to solve the pressure system of the first stage. Because AMG is designed for efficient solution of (near) elliptic equations, we pay special attention to preserving the natural near-elliptic character of the pressure field when constructing $A_{p}$. In the second stage, we operate on the entire linear system to reduce the error, with emphasis on the high frequency components. A local preconditioner, such as ILU, is usually a reasonable choice for the second stage.

\section{IMPES Formulation}

Our focus here is on solving FIM problems with coupled wells. The first stage of the CPR method is quite similar to the IMPES formulation; as a result, a detailed understanding of the behavior of IMPES problems with coupled wells would be quite valuable for dealing effectively with the more complex FIM model. In this section we analyze the properties of the IMPES pressure matrix, and we discuss the performance of several AMG-based preconditioners for systems with coupled wells.

\section{PART I: Reservoir Equations}

In order to keep the discussion relatively simple, but without loss of generality, we consider the case where each cell has only two neighboring cells. The governing equations of the oil and water components, written in residual form, are (Aziz et al. 2003): 


$$
\begin{aligned}
& R_{o, i}=-T_{o, i-1 / 2}\left(P_{o, i-1}-P_{o, i}\right)-T_{o, i+1 / 2}\left(P_{o, i+1}-P_{o, i}\right)-T_{o}^{w}\left(P^{w}+G-P_{o, i}\right) \\
& +\frac{V_{i}}{\Delta t} \Delta_{t}\left(\phi S_{o} b_{o}\right)_{i}=0 \\
& R_{w, i}=-T_{w, i-1 / 2}\left(P_{w, i-1}-P_{w, i}\right)-T_{w, i+1 / 2}\left(P_{w, i+1}-P_{w, i}\right)-T_{w}^{w}\left(P^{w}+G-P_{w, i}\right) \\
& +\frac{V_{i}}{\Delta t} \Delta_{t}\left(\phi S_{w} b_{w}\right)_{i}=0
\end{aligned}
$$

where $R$ is the residual, T is the transmissibility. $P$ and $S$ denote pressure and saturation, respectively. $G$ is the hydraulic head from the pressure reference point to the current completion (perforation) location, and $V_{i}$ is the bulk volume of cell $i$. Subscripts $w$ and $o$, stand for water and oil, respectively; superscript $w$ stands for the well. For example, $T_{w}^{w}$ is the well transmissibility of water. The reciprocal of the formation volume factor (FVF) is $b$, and $\Delta t$ is the timestep size.

After linearization of Eqs. 2-10 and 2-11 using the Newton-Raphson method, we obtain the Jacobian matrix, which has two linear equations per cell. We write the equations for one cell as:

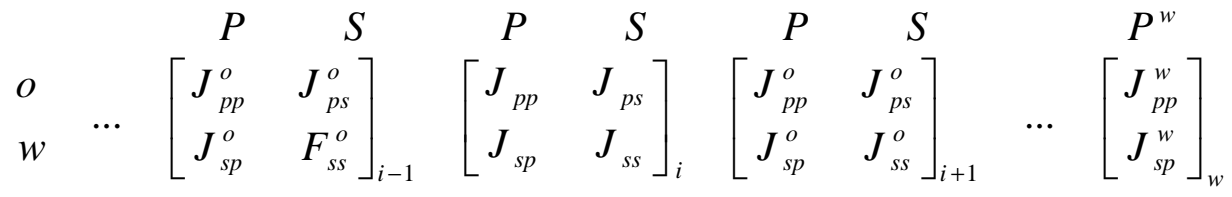

In Eqs. 2-10 and 2-11, the oil component equation is always selected as the primary equation and the water component equation as the secondary equation. The first subscript $p$ stands for primary and the second for pressure; the first subscript $s$ stands for secondary and the second for saturation. The superscript $o$ denotes an off-diagonal entry. The diagonal $J$ entries have a flux component and an accumulation component. The off-diagonal entries only have flux components. So, we can write Eq. 2-12:

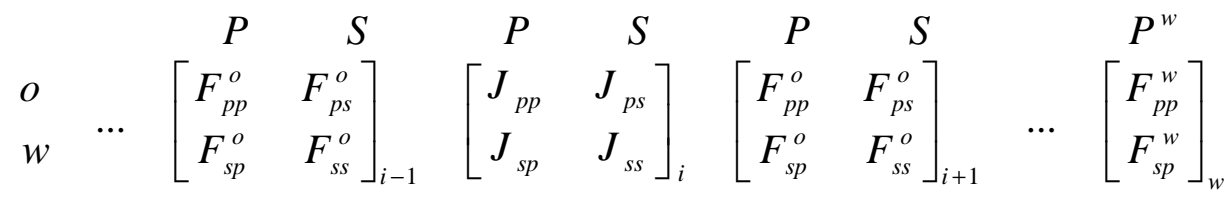


For example, $F_{p s}^{o}$ denotes the derivative of the flux to the neighboring cell in the primary equation with respect to the neighbor's saturation. Eq. 2-13 is for one cell. If we combine the equations for all the cells, we obtain the FIM system. A simple 2D problem with a multiblock well and the incidence representation of the FIM Jacobian matrix for a two-phase system are shown in Fig. 2-2.

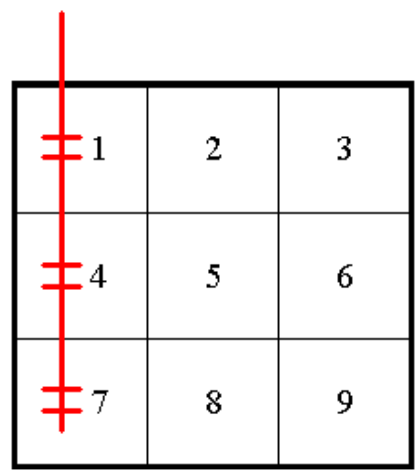

\begin{tabular}{|c|c|c|c|c|c|c|c|c|c|c|}
\hline & P S & P S & P S & P S & P S & P S & P S & P S & P S & $\mathrm{P}$ \\
\hline 1 & $\begin{array}{l}\text { X X } \\
\text { X X }\end{array}$ & $\begin{array}{l}\mathbf{X} X \\
\mathbf{X X}\end{array}$ & & $\begin{array}{l}\text { X X } \\
\text { X X }\end{array}$ & & & & & & $\begin{array}{l}\mathbf{X} \\
\mathbf{X}\end{array}$ \\
\hline 2 & $\begin{array}{l}\text { X X } \\
\text { X X }\end{array}$ & $\begin{array}{l}\mathbf{X} X \\
\mathbf{X ~ X}\end{array}$ & $\begin{array}{l}\mathbf{X} X \\
\mathbf{X ~ X}\end{array}$ & & $\begin{array}{l}\text { X X } \\
\text { X X }\end{array}$ & & & & & \\
\hline 3 & & $\begin{array}{l}\text { X X } \\
\text { X X }\end{array}$ & $\begin{array}{l}X X \\
X X\end{array}$ & & & $\begin{array}{l}\mathbf{X X} \\
\text { X X }\end{array}$ & & & & \\
\hline 4 & $\begin{array}{l}\text { X X } \\
\text { X X }\end{array}$ & & & $\begin{array}{l}\text { X X } \\
\text { X X }\end{array}$ & $\begin{array}{l}\text { X X } \\
\text { X X }\end{array}$ & & $\begin{array}{l}\text { X X } \\
\text { X X }\end{array}$ & & & $\begin{array}{l}\mathbf{X} \\
\mathbf{X}\end{array}$ \\
\hline 5 & & $\begin{array}{l}\mathbf{X} X \\
\mathbf{X ~ X}\end{array}$ & & $\begin{array}{l}\text { X X } \\
\text { X X }\end{array}$ & $\begin{array}{l}\text { X X } \\
\text { X X }\end{array}$ & $\begin{array}{l}\text { X X } \\
\text { X X }\end{array}$ & & $\begin{array}{l}\text { X X } \\
\text { X X }\end{array}$ & & \\
\hline 6 & & & $\begin{array}{l}X X \\
X X\end{array}$ & & $\begin{array}{l}\text { X X } \\
\text { X X }\end{array}$ & $\begin{array}{l}\mathbf{X} \mathbf{X} \\
\mathbf{X} \mathbf{X}\end{array}$ & & & $\begin{array}{l}X X \\
X X\end{array}$ & \\
\hline 7 & & & & $\begin{array}{l}\text { X X } \\
\text { X X }\end{array}$ & & & $\begin{array}{l}X X \\
X X\end{array}$ & $\begin{array}{l}\text { X X } \\
\text { X X }\end{array}$ & & $\begin{array}{l}\mathbf{X} \\
\mathbf{X}\end{array}$ \\
\hline 8 & & & & & $\begin{array}{l}\text { X X } \\
\text { X X }\end{array}$ & & $\begin{array}{l}\text { X X } \\
\text { X X }\end{array}$ & $\begin{array}{l}\text { X X } \\
\text { X X }\end{array}$ & $\begin{array}{l}\mathbf{X} X \\
\mathbf{X ~ X}\end{array}$ & \\
\hline 9 & & & & & & $\begin{array}{l}\text { X X } \\
\text { X X }\end{array}$ & & $\begin{array}{l}\text { X X } \\
\text { X X }\end{array}$ & $\begin{array}{l}\text { X X } \\
\text { X X }\end{array}$ & \\
\hline & X X & & & $\overline{X X}$ & & & $\overline{X X}$ & & & $\overline{\mathbf{X}}$ \\
\hline
\end{tabular}

Fig. 2-2: A 3x3 FIM system with one well (after Aziz et al. 2003)

In order to reduce the matrix in Fig. 2-2 to an IMPES system, we treat the transmissibility between cells explicitly. Then the flux terms between cells are no longer functions of saturation. However, the well terms are always treated implicitly. Consequently, Eq. 2-13 reduces to Eq. 2-14:

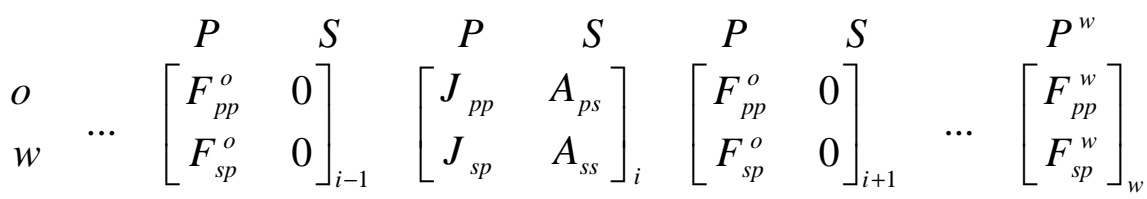

With

$$
C_{o}=\partial b_{o} / \partial P>0
$$




$$
C_{w}=\partial b_{w} / \partial P>0
$$

the accumulation terms for oil and water can be expressed as Eq. 2-17 and Eq. 2-18 (Aziz, et al. 2003):

$$
\begin{aligned}
& A_{o}=\frac{V \phi}{\Delta t}\left[\left(b_{o} S_{o}\right)^{n+1}-\left(b_{o} S_{o}\right)^{n}\right] \\
& A_{w}=\frac{V \phi}{\Delta t}\left[\left(b_{w} S_{w}\right)^{n+1}-\left(b_{w} S_{w}\right)^{n}\right]
\end{aligned}
$$

The derivatives of accumulation terms with respect to pressure and saturation take the following form:

$$
\begin{aligned}
& A_{p p}=\partial A_{o} / \partial P=\frac{V \phi}{\Delta t}\left(C_{o} S_{o}\right)^{n+1} \\
& A_{s p}=\partial A_{w} / \partial P=\frac{V \phi}{\Delta t}\left(C_{w} S_{w}\right)^{n+1} \\
& A_{p s}=\partial A_{o} / \partial S_{w}=-\frac{V \phi}{\Delta t} b_{o}{ }^{n+1} \\
& A_{s s}=\partial A_{w} / \partial S_{w}=\frac{V \phi}{\Delta t} b_{w}{ }^{n+1}
\end{aligned}
$$

The derivatives of flux terms with respect to pressure can be written as follows:

$$
\begin{gathered}
F_{p p}=\sum\left[T_{o}+T_{o}^{w}-\frac{\partial T_{o}^{w}}{\partial P}\left(P^{w}+G-P_{o, i}\right)\right] \\
F_{s p}=\sum\left[T_{w}+T_{w}^{w}-\frac{\partial T_{w}^{w}}{\partial P}\left(P^{w}+G-P_{o, i}\right)\right] \\
F_{p p}{ }^{o}=-T_{o} \\
F_{s p}{ }^{o}=-T_{w} \\
F_{p p}^{w}=-T_{o}^{w}
\end{gathered}
$$




$$
F_{s p}^{w}=-T_{w}^{w}
$$

Local Gaussian elimination is used to manipulate Eq. 2-14:

$$
\begin{gathered}
P \\
w \\
w
\end{gathered} \quad \cdots \quad\left[\begin{array}{cc}
F_{p p}^{o}-A_{p s} A_{s s}^{-1} F_{s p}^{o} & 0 \\
F_{s p}^{o} & 0
\end{array}\right]_{i-1} \quad\left[\begin{array}{cc}
J_{p p}-A_{p s} A_{s s}^{-1} J_{s p} & 0 \\
J_{s p} & A_{s s}
\end{array}\right]_{i} \quad \ldots \quad \ldots\left[\begin{array}{c}
P^{w} \\
F_{p p}^{w}-A_{p s} A_{s s}^{-1} F_{s p}^{w} \\
F_{s p}^{w}
\end{array}\right]_{w}
$$

The corresponding system is shown in Fig. 2-3. The pressure matrix can be extracted as shown in Fig. 2-4. The fill-in terms, shown as 'o' in Fig. 2-4 are due to the fully implicit

\begin{tabular}{|c|c|c|c|c|c|c|c|c|c|c|}
\hline & $\mathrm{PS}$ & PS & $\mathrm{PS}$ & $\mathrm{PS}$ & $\mathrm{PS}$ & $\mathrm{PS}$ & $\mathrm{PS}$ & $\mathrm{PS}$ & $\mathrm{PS}$ & $\mathrm{P}$ \\
\hline 1 & $\begin{array}{l}\mathbf{X} \\
\mathrm{XX}\end{array}$ & $\begin{array}{l}\mathbf{X} \\
X\end{array}$ & & $\begin{array}{l}\mathbf{X} \\
X\end{array}$ & & & & & & \begin{tabular}{|l|}
$\mathbf{X}$ \\
$\mathrm{X}$
\end{tabular} \\
\hline 2 & $\begin{array}{l}\mathbf{X} \\
\mathrm{X}\end{array}$ & $\begin{array}{l}\mathbf{X} \\
\mathrm{XX}\end{array}$ & $\begin{array}{l}\mathbf{X} \\
X\end{array}$ & & $\begin{array}{l}\mathbf{X} \\
\mathrm{X}\end{array}$ & & & & & \\
\hline 3 & & $\begin{array}{l}\mathbf{X} \\
\mathrm{X}\end{array}$ & $\begin{array}{l}\mathbf{X} \\
\mathrm{XX}\end{array}$ & & & $\begin{array}{l}\mathbf{X} \\
\mathrm{X}\end{array}$ & & & & \\
\hline 4 & $\begin{array}{l}\mathbf{X} \\
\mathrm{X}\end{array}$ & & & $\begin{array}{l}\mathbf{X} \\
\mathrm{XX}\end{array}$ & $\begin{array}{l}\mathbf{X} \\
\mathbf{X}\end{array}$ & & $\begin{array}{l}\mathbf{X} \\
\mathrm{X}\end{array}$ & & & \begin{tabular}{|l}
$\mathbf{X}$ \\
$\mathrm{X}$
\end{tabular} \\
\hline 5 & & $\begin{array}{l}\mathbf{X} \\
\mathrm{X}\end{array}$ & & $\begin{array}{l}\mathbf{X} \\
\mathrm{X}\end{array}$ & $\begin{array}{l}\mathbf{X} \\
\text { X X }\end{array}$ & $\begin{array}{l}\mathbf{X} \\
\mathbf{X}\end{array}$ & & $\begin{array}{l}\mathbf{X} \\
\mathrm{X}\end{array}$ & & \\
\hline 6 & & & $\begin{array}{l}\mathbf{X} \\
\mathrm{X}\end{array}$ & & $\begin{array}{l}\mathrm{X} \\
\mathrm{X}\end{array}$ & $\begin{array}{l}\text { XX } \\
\text { XX }\end{array}$ & & & $\begin{array}{l}\mathbf{X} \\
\mathrm{X}\end{array}$ & \\
\hline 7 & & & & $\begin{array}{l}\mathbf{X} \\
\mathrm{X}\end{array}$ & & & $\begin{array}{l}\mathbf{X} \\
\text { X X }\end{array}$ & $\begin{array}{l}\mathbf{X} \\
\mathrm{X}\end{array}$ & & $\begin{array}{l}\mathbf{x} \\
\mathbf{X}\end{array}$ \\
\hline 8 & & & & & $\begin{array}{l}\mathbf{X} \\
\mathbf{X}\end{array}$ & & $\begin{array}{l}\mathbf{X} \\
\mathrm{X}\end{array}$ & \begin{tabular}{|l}
$\mathbf{X}$ \\
$\mathrm{X} \mathrm{X}$
\end{tabular} & $\begin{array}{l}\mathbf{X} \\
\mathbf{X}\end{array}$ & \\
\hline 9 & & & & & & $\begin{array}{l}\mathbf{X} \\
\mathrm{X}\end{array}$ & & $\begin{array}{l}\mathbf{X} \\
\mathbf{X}\end{array}$ & $\begin{array}{l}\mathbf{X} \\
\mathrm{XX}\end{array}$ & \\
\hline & X X & & & XX & & & $\mathrm{XX}$ & & & $\mathbf{x}$ \\
\hline
\end{tabular}
treatment of the well.

Fig. 2-3: Matrix after local Gaussian elimination 


\begin{tabular}{|c|c|c|c|c|c|c|c|c|c|c|}
\hline & $\mathrm{P}$ & $\mathrm{P}$ & $\mathrm{P}$ & $\mathrm{P}$ & $\mathrm{P}$ & $\mathrm{P}$ & $\mathrm{P}$ & $\mathrm{P}$ & $\mathrm{P}$ & $\mathrm{P}$ \\
\hline 1 & $\mathbf{X}$ & $\mathbf{X}$ & & $\mathbf{X}$ & & & & & & $\mathbf{X}$ \\
\hline 2 & $\mathbf{X}$ & $\mathbf{X}$ & $\mathbf{X}$ & & $\mathbf{X}$ & & & & & \\
\hline 3 & & $\mathbf{X}$ & $\mathbf{X}$ & & & $\mathbf{X}$ & & & & \\
\hline 4 & $\mathbf{X}$ & & & $\mathbf{X}$ & $\mathbf{X}$ & & $\mathbf{X}$ & & & $\mathbf{X}$ \\
\hline 5 & & $\mathbf{X}$ & & $\mathbf{X}$ & $\mathbf{X}$ & $\mathbf{X}$ & & $\mathbf{X}$ & & \\
\hline 6 & & & $\mathbf{X}$ & & $\mathbf{X}$ & $\mathbf{X}$ & & & $\mathbf{X}$ & \\
\hline 7 & & & & $\mathbf{X}$ & & & $\mathbf{X}$ & $\mathbf{X}$ & & $\mathbf{X}$ \\
\hline 8 & & & & & $\mathbf{X}$ & & $\mathbf{X}$ & $\mathbf{X}$ & $\mathbf{X}$ & \\
\hline 9 & & & & & & $\mathbf{X}$ & & $\mathbf{X}$ & $\mathbf{X}$ & \\
\hline & $\mathbf{X}$ & O & & $\mathbf{X}$ & $\mathbf{O}$ & & $\mathbf{X}$ & $\mathbf{O}$ & & $\mathbf{X}$ \\
\hline
\end{tabular}

Fig. 2-4: IMPES pressure matrix

The terms in the pressure matrix can be written as follows:

$$
\begin{gathered}
A_{p}{ }^{d}=\left(A_{p p}+F_{p p}\right)-A_{p s} \cdot A_{s s}{ }^{-1} \cdot\left(A_{s p}+F_{s p}\right) \\
A_{p}{ }^{o}=F_{p p}{ }^{o}-A_{p s} \cdot A_{s s}{ }^{-1} \cdot F_{s p}{ }^{o} \\
A_{p}{ }^{w}=F_{p p}{ }^{w}-A_{p s} \cdot A_{s s}{ }^{-1} \cdot F_{s p}{ }^{w}
\end{gathered}
$$

Here, $A_{p}^{d}$ is the diagonal term of the pressure matrix, $A_{p}^{o}$ is the off-diagonal term of the pressure matrix and $A_{p}^{w}$ stands for the well term.

We are interested in the sign of the row sum of the pressure matrix.

$$
\text { Rowsum }=\frac{V \phi}{\Delta t}\left[C_{o}\left(1-S_{w}\right)+\frac{b_{o}}{b_{w}}\left(C_{w} S_{w}\right)\right]-\left(\frac{\partial T_{o}^{w}}{\partial P}+\frac{b_{o}}{b_{w}} \frac{\partial T_{w}^{w}}{\partial P}\right)\left(P^{w}-P\right)
$$

The first term of Eq. 2-33 is the accumulation term, which is positive, and the second term is the well term. Eq. 2-33 has nothing to do with the flux between cells. If the phase compressibilities of the system are in the normal range, the accumulation term is much larger 
than the well term, so the row sum is positive. If the system is incompressible, we can ignore the first term and the well term becomes dominant.

The well transmissibilities are defined as (Aziz et al. 2003):

$$
\begin{gathered}
T_{o}^{w}=\frac{k k_{r o} b_{o}}{\mu_{o}} W I \\
T_{w}^{w}=\frac{k k_{r w} b_{w}}{\mu_{w}} W I
\end{gathered}
$$

where $k$ is the permeability of the cell, $k_{r o}$ and $k_{r w}$ are the relative permeabilities of oil and water, respectively, and $\mu_{o}$ and $\mu_{w}$ are the viscosities of the oil and water phases. The well index is represented by $W I$. We then obtain

$$
\text { Rowsum }=-b_{o} W I\left[k_{r o} \frac{\partial}{\partial P}\left(\frac{1}{\mu_{o}}\right)+k_{r w} \frac{\partial}{\partial P}\left(\frac{1}{\mu_{w}}\right)\right]\left(P^{\text {well }}-P\right)
$$

Generally, $\frac{\partial}{\partial P}\left(\frac{1}{\mu_{o}}\right)<0$ and $\frac{\partial}{\partial P}\left(\frac{1}{\mu_{w}}\right)<0$, so the sign of $P^{\text {well }}-P$, (i.e. the well type) determines the sign of the row sum. It could be negative if the well is a producer. If the system is incompressible, and there is no well perforation in the cell, then the row sum is zero.

\section{PART II: Well Equations}

Here, we analyze the well terms in the Jacobian matrix. The objective is to see how the IMPES procedure will effect the row sum of the well equations. We focus on the row-sum property.

Assume we have a production well with a specified oil rate, we get 


$$
R^{w}=\sum_{i=1}^{N P} T_{o}^{w}\left(P^{w}-P_{o, i}\right)+q_{o}=0
$$

where $q_{o}$ is the specified oil rate, $R^{w}$ is residual of the well equation, and $N P$ is the number of perforations of the well.

Linearization of Eq. 2-37 adds one row to the matrix shown in Fig. 2-2.

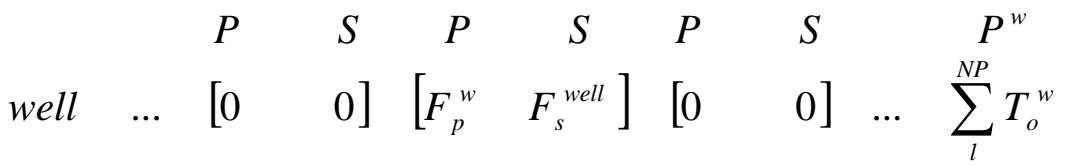

$$
\begin{aligned}
& F_{p}^{w}=-T_{o}^{w}-\frac{\partial T_{o}^{w}}{\partial P}\left(P_{i}-P^{w}\right) \\
& F_{s}^{w}=-\frac{\partial T_{o}^{w}}{\partial S_{w}}\left(P_{i}-P^{w}\right)
\end{aligned}
$$

To eliminate the saturation derivation in the well equation, new pressure derivative terms are introduced in the neighbors of the current perforated well cell:

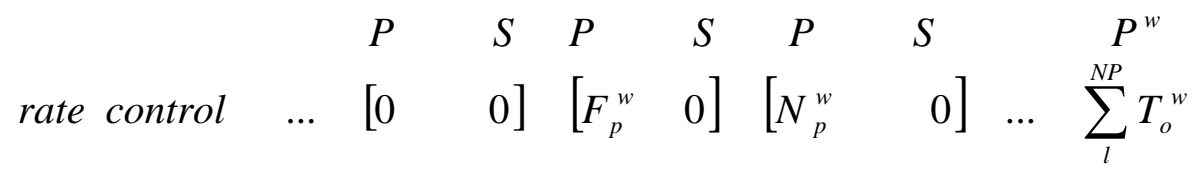

where, the new fill-in term is

$$
N_{p}^{w}=-\frac{\partial T_{o}^{w}}{\partial S_{w}}\left(P_{i}-P^{w}\right) A_{s s}^{-1} T_{w}
$$

We notice that $\frac{\partial T_{o}^{w}}{\partial S_{w}}$ is always negative. So $N_{p}^{w}$ is positive when the well is a producer. This is a positive off-diagonal term, which can affect the performance of AMG negatively. Generally, this term is quite small.

The row-sum of the well equation for a producer is 


$$
\begin{aligned}
& \text { Rowsum }=\sum_{i}^{N P}\left\{\frac{\partial T_{o}^{w}}{\partial S}\left(P_{i}-P^{w}\right) A_{s s}^{-1}\left[A_{s p}-\frac{\partial T_{w}^{w}}{\partial P}\left(P^{w}-P_{i}\right)\right]\right\} \\
& -\sum_{i}^{N P} \frac{\partial T_{o}^{w}}{\partial P}\left(P_{i}-P^{w}\right)
\end{aligned}
$$

If the system is compressible, we have

$$
A_{s p}=\frac{V \phi}{\Delta t}\left(C_{w} S_{w}\right)^{n+1}>>\frac{\partial T_{w}^{w}}{\partial P}\left(P^{w}-P_{i}\right)
$$

Then,

$$
\text { Rowsum } \approx \sum_{i}^{N P}\left(\frac{\partial T_{o}^{w}}{\partial S_{w}} \frac{C_{w} S_{w}}{b_{w}}-\frac{\partial T_{o}^{w}}{\partial P}\right)\left(P_{i}-P^{w}\right)
$$

$\frac{\partial T_{o}^{w}}{\partial S_{w}}$ is always negative and $\frac{\partial T_{o}^{w}}{\partial P}$ is positive, since the FVF usually is more important than the viscosity. Hence, we are likely to have a negative row sum when the well is a producer.

If we have an incompressible system, the row sum can be written as Eq. 2-46:

$$
\begin{aligned}
& \text { Rowsum }=\sum_{i}^{N P}\left\{\frac{\partial T_{o}^{w}}{\partial S_{w}}\left(P_{i}-P^{\text {well }}\right) A_{s s}^{-1}\left[-\frac{\partial T_{w}^{w}}{\partial P}\left(P^{w}-P_{i}\right)\right]\right\} \\
& -\sum_{i}^{N P} \frac{\partial T_{o}^{w}}{\partial P}\left(P_{i}-P^{w}\right)
\end{aligned}
$$

Generally, $\frac{\partial T_{w}^{w}}{\partial P}$ is negative, so the first term is positive. The sign of second term depends on the type of the well. If the system is incompressible and viscosity is not a function of pressure, the row sum is zero.

Using a similar procedure, we can derive the row-sum of the well equation for a water injector as follows: 


$$
\begin{aligned}
& \text { Rowsum }=\sum_{i}^{N P}\left\{\frac{\partial T_{T}^{w}}{\partial S_{w}}\left(P_{i}-P^{w}\right) A_{s s}^{-1}\left[A_{s p}-\frac{\partial T_{T}^{w}}{\partial P}\left(P^{w}-P_{i}\right)\right]\right\} \\
& -\sum_{i}^{N P} \frac{\partial T_{T}^{w}}{\partial P}\left(P_{i}-P^{w}\right)
\end{aligned}
$$

where $T_{T}^{w}$ is the total transmissibility of the perforations. Eq. 2-47 is slightly different from the equation for a producer.

Table 2-1: Summary of properties of the IMPES matrix

\begin{tabular}{|c|c|c|c|}
\hline Equations & Well Type & Diagonal & Row Sum \\
\hline \multicolumn{4}{|c|}{ Incompressible System } \\
\hline Reservoir Equ. & Producer & + & - \\
\cline { 2 - 4 } & Injector & + & + \\
\hline \multirow{2}{*}{ Well Equ. } & Producer & + & 0 \\
\cline { 2 - 4 } & Injector & + & 0 \\
\hline \multirow{4}{*}{ Reservoir Equ. } & Coducressible System & + \\
\cline { 2 - 4 } & Injector & + & + \\
\hline \multirow{2}{*}{ Well Equ. } & Producer & + & - \\
\cline { 2 - 4 } & Injector & + & + \\
\hline
\end{tabular}

Table 2-1 summarizes the properties of the IMPES pressure matrix for different conditions. For the reservoir equations, the row sum is positive as long as the system is compressible. However, if rate controlled production wells are present in a compressible system, we may get a negative row sum for the well equations, which may cause problems for the AMG solver.

\section{Row-Sum Preconditioner}

The pressure matrix can be divided into four parts, $A_{r r}, A_{r w}, A_{w r}$ and $A_{w w}$ as shown in Fig. 2-5, where the first subscript refers to the equation, and the second subscript refers to the variable. Here, $r$ and $w$ denote quantities related to the reservoir and wells, respectively. Since implicit wells may cause problems for $\mathrm{AMG}$, we can use the rate constraint to 
eliminate the well terms from the reservoir equations. The resulting Schur complement matrix has reservoir equations only, but with additional fill-in (see $A_{r r}{ }^{*}$ in Fig. 2-5):

$$
A_{r r}^{*}=A_{r r}-A_{r w} \cdot A_{w w}^{-1} \cdot A_{w r}
$$

The second term on the right hand side has the same size as $A_{r r}$; however, $A_{r r}^{*}$ has new fill-in terms compared with $A_{r r}$.

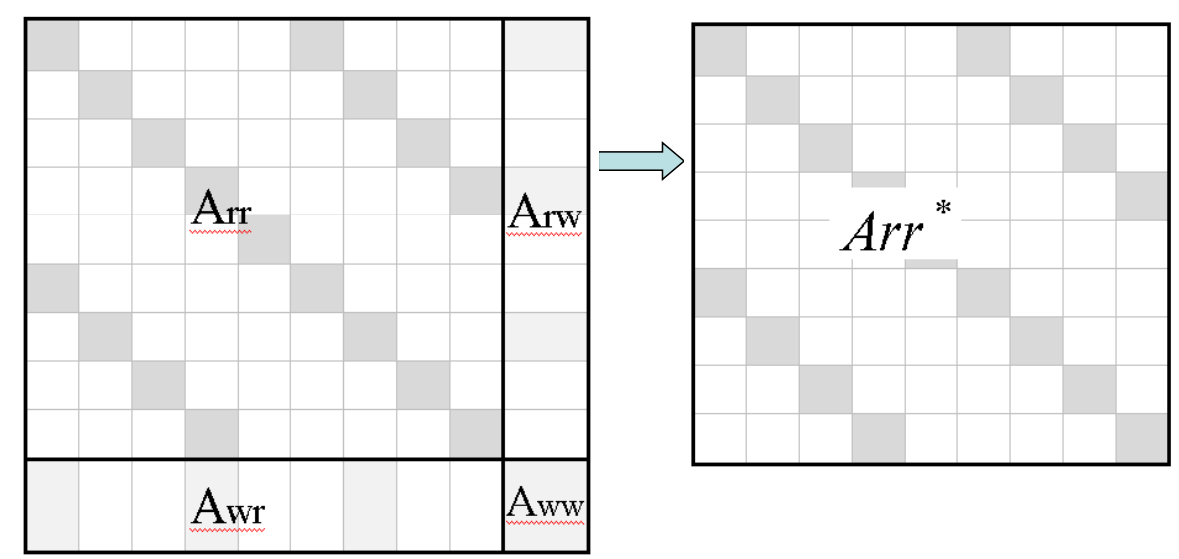

Fig. 2-5: Structure of IMPES pressure matrix and reduced system

The explicit form of the coefficient matrix is not needed by GMRES since only the result of applying the matrix to a vector (i.e. matrix-vector multiplication) is of interest.

$$
A_{r r}^{*} \cdot \vec{v}=A_{r r} \cdot \vec{v}-A_{r w} \cdot A_{w w}^{-1} \cdot A_{w r} \cdot \vec{v}
$$

As shown in Eq. 2-49, we can get the result of matrix-vector multiplication $A_{r r}^{*} \cdot \vec{v}$ without knowing the explicit form of the coefficient matrix $A_{r r}^{*}$.

AMG needs every element in the matrix. Table 2-2.lists three different matrices we use as precondtioners. 
Table 2-2: Summary of the properties of pressure matrix

\begin{tabular}{|c|c|}
\hline & AMG Pre \\
\hline 1 & $S=A_{r r}-A_{r w} \cdot A_{w w}^{-1} \cdot A_{w r}$ \\
\hline 2 & $A_{r r}$ \\
\hline 3 & $A_{r r}-\operatorname{diag}\left(R S\left(A_{r w} \cdot A_{w w}^{-1} \cdot A_{w r}\right)\right)$ \\
\hline
\end{tabular}

The first choice in Table 2-2 is the Schur complement, which can be very expensive to compute. In the second choice, we simply drop the well terms and use $A_{r r}$. This can be a good preconditioner if, for example, the well indices are relatively small. The third choice is what we call the row-sum preconditioner, which is the preferred method.

The row-sum preconditioner is an approximation to the Schur complement:

$$
R S\left(A_{r w} \cdot A_{w w}^{-1} \cdot A_{w r}\right)=A_{r w} \cdot A_{w w}^{-1} \cdot A_{w r} \cdot \vec{e}
$$

where $\vec{e}$ is the vector with all elements equal to unity. We do not need to work out $A_{r w} \cdot A_{w w}^{-1} \cdot A_{w r}$, we just perform the operations in Eq. 2-50 from right to left. The interim results are always vectors, and the computational cost is low. We subtract the row-sum result of $A_{r w} \cdot A_{w w}^{-1} \cdot A_{w r}$ from the diagonal of $A_{r r}$.

The performance of the three preconditioners is analyzed by considering the problem shown in Fig. 2-6. It is a 3D oil-water system with 141,900 cells (110x30x43). There are 11 fully penetrating vertical wells in the reservoir. Ten injection wells with water rate control are located near a corner. There is a production well with BHP control in the middle of the reservoir. 


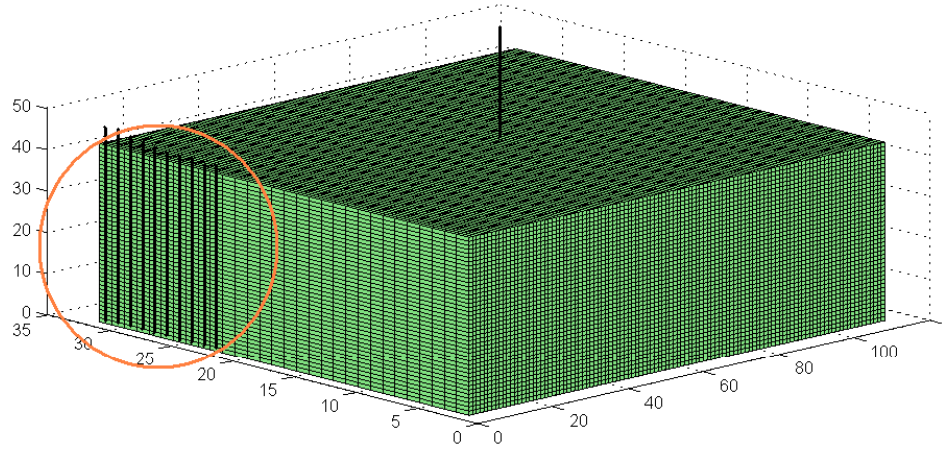

Fig. 2-6: Reservoir map of test case

We solved the test case with the IMPES method. The solver is GMRES with the three preconditioners listed in Table 2-2. Figs. 2-7 to 2-9 show the linear solver performance. In each figure, there are several lines. Each line represents the linear system for a Newton iteration.

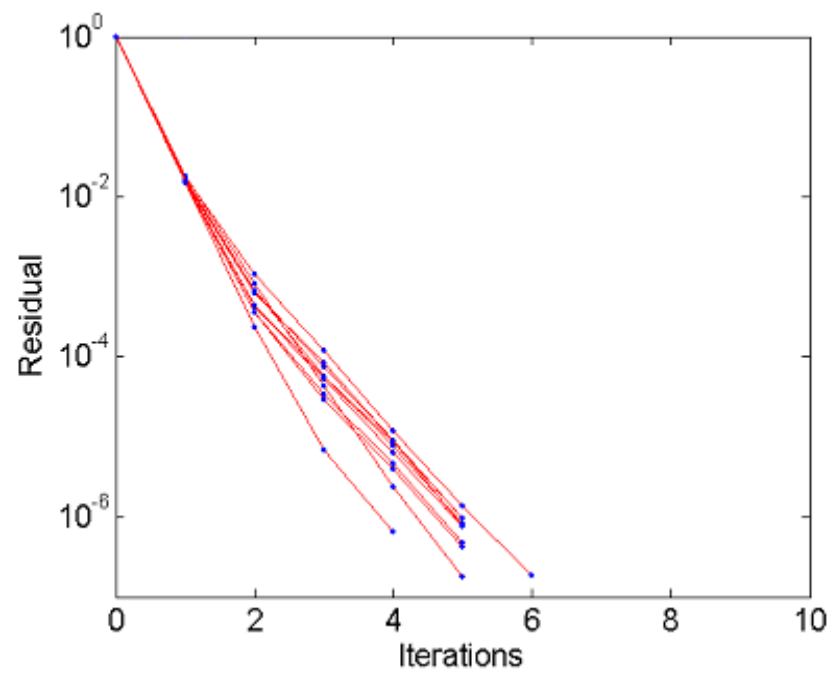

Fig. 2-7: Residual reduction for GMRES with the Schur complement preconditioner 


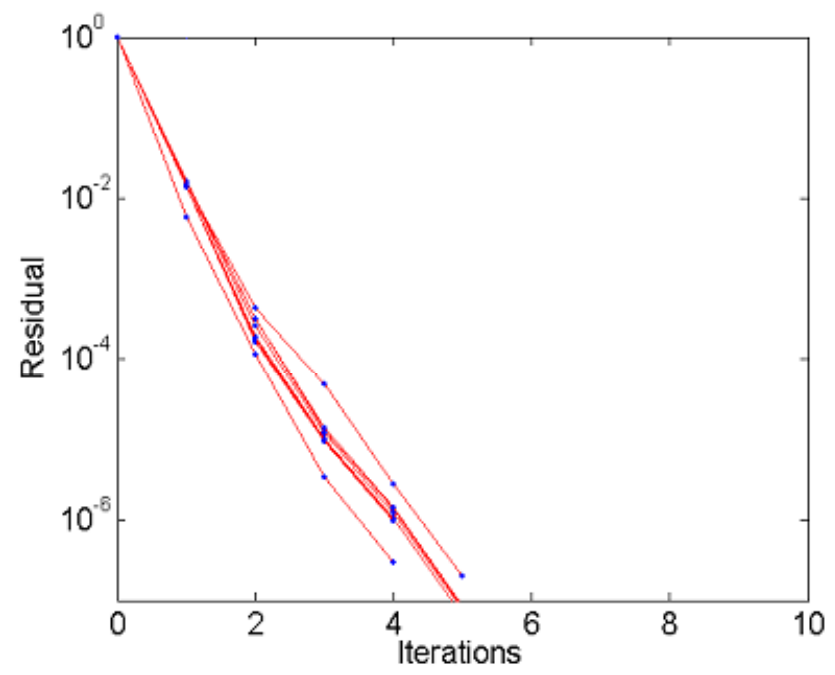

Fig. 2-8: Residual reductions for GMRES with the $A_{r r}$ preconditioner

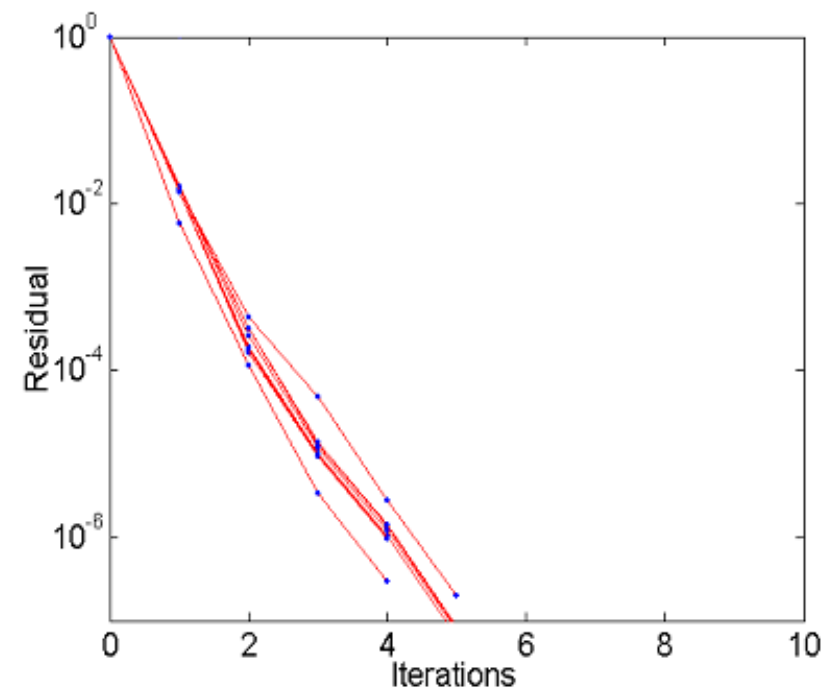

Fig. 2-9: Residual reductions for GMRES with the row-sum preconditioner

From Figs. 2-7 to 2-9, we see that the three preconditioners perform well for this problem. The average number of iterations to reach convergence (within the specified tolerance) per linear solve is about five.

The second test case is very similar to the first one, but the well roles are switched. The corner wells are producers with rate control, and the well in the middle is an injector with 
BHP control. Figs. 2-10 to 2-12 show the performance of the three preconditioners for this case. The Schur complement preconditioner still works quite well because it has full information about the matrix. The $A_{r r}$ preconditioner performs poorly in this case. On the other hand, the performance of the row-sum preconditioner is quite good.

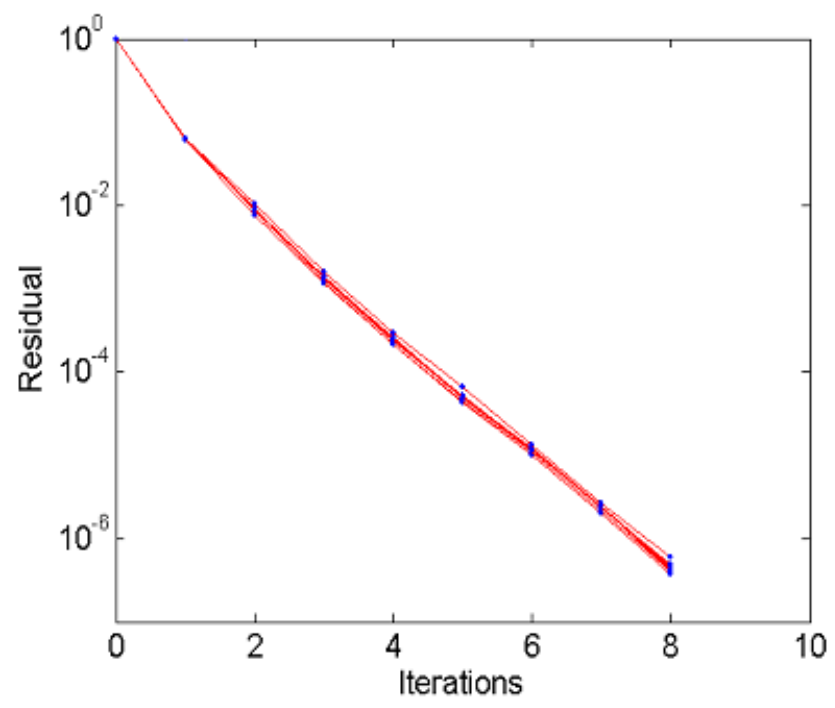

Fig. 2-10: Residual reductions for GMRES with Schur complement preconditioner

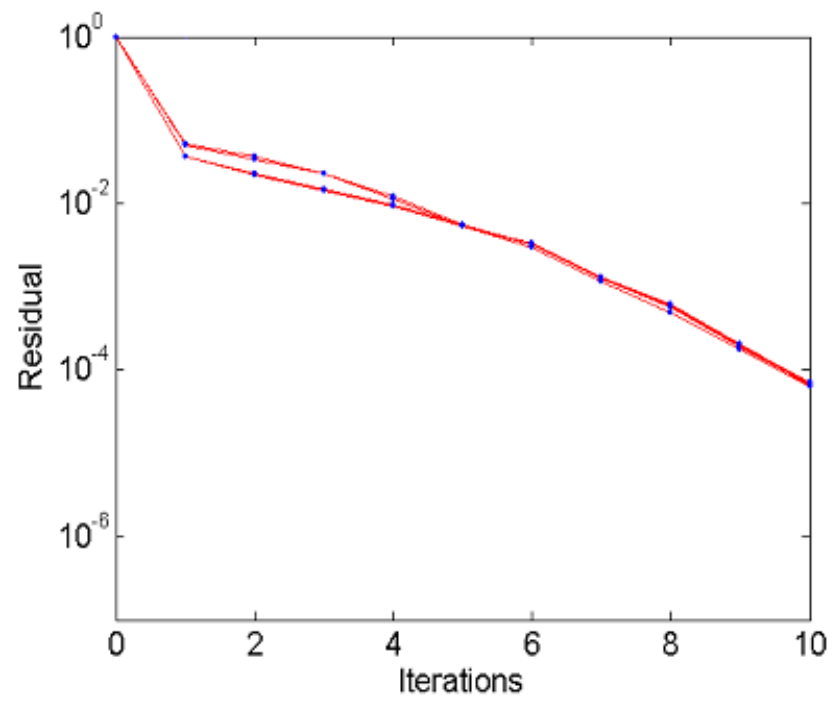

Fig. 2-11: Residual reductions for GMRES with Arr preconditioner 


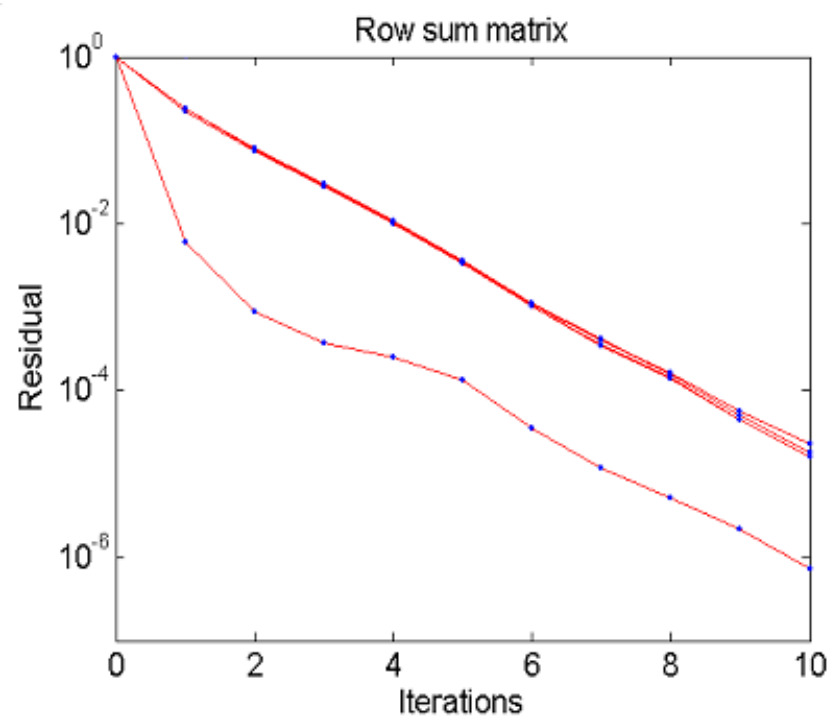

Fig. 2-12: Residual reductions for GMRES with row sum preconditioner

The two test cases demonstrate that at the row-sum preconditioner works well, and that a rate controlled multi-block production well can cause problems for AMG.

\section{Application to FIM Formulation}

Due to its unconditional stability, FIM is widely used in reservoir simulation. In large-scale problem, most of the run-time is spent on the linear solver. As a result, improving the solver performance leads to significant gains in the overall performance of the simulator.

We study three FIM preconditioners: ILU, CPR with True-IMPES, and CPR with QuasiIMPES. We illustrate the behavior of these preconditioners using a simple problem. The test is based on six different model sizes for a homogeneous, 3D, oil-water system (see Table 23). 
Table 2-3: Dimensions of the six cases

\begin{tabular}{|c|c|r|}
\hline case & Dimension & \multicolumn{1}{c|}{ Cells } \\
\hline 1 & $15 \times 55 \times 2$ & 1,650 \\
\hline 2 & $30 \times 110 \times 2$ & 6,600 \\
\hline 3 & $30 \times 110 \times 10$ & 33,000 \\
\hline 4 & $30 \times 110 \times 20$ & 66,000 \\
\hline 5 & $30 \times 110 \times 30$ & 99,000 \\
\hline 6 & $30 \times 110 \times 40$ & 132,000 \\
\hline
\end{tabular}

There are two wells in the reservoir. The production well with BHP control is located at one corner; the injection well with rate control is in the middle of the reservoir. Both wells penetrate all the layers. We report on the performance of the different preconditioners for each case. Fig. 2-13 shows the CPU time spent on the linear solver versus the model size.

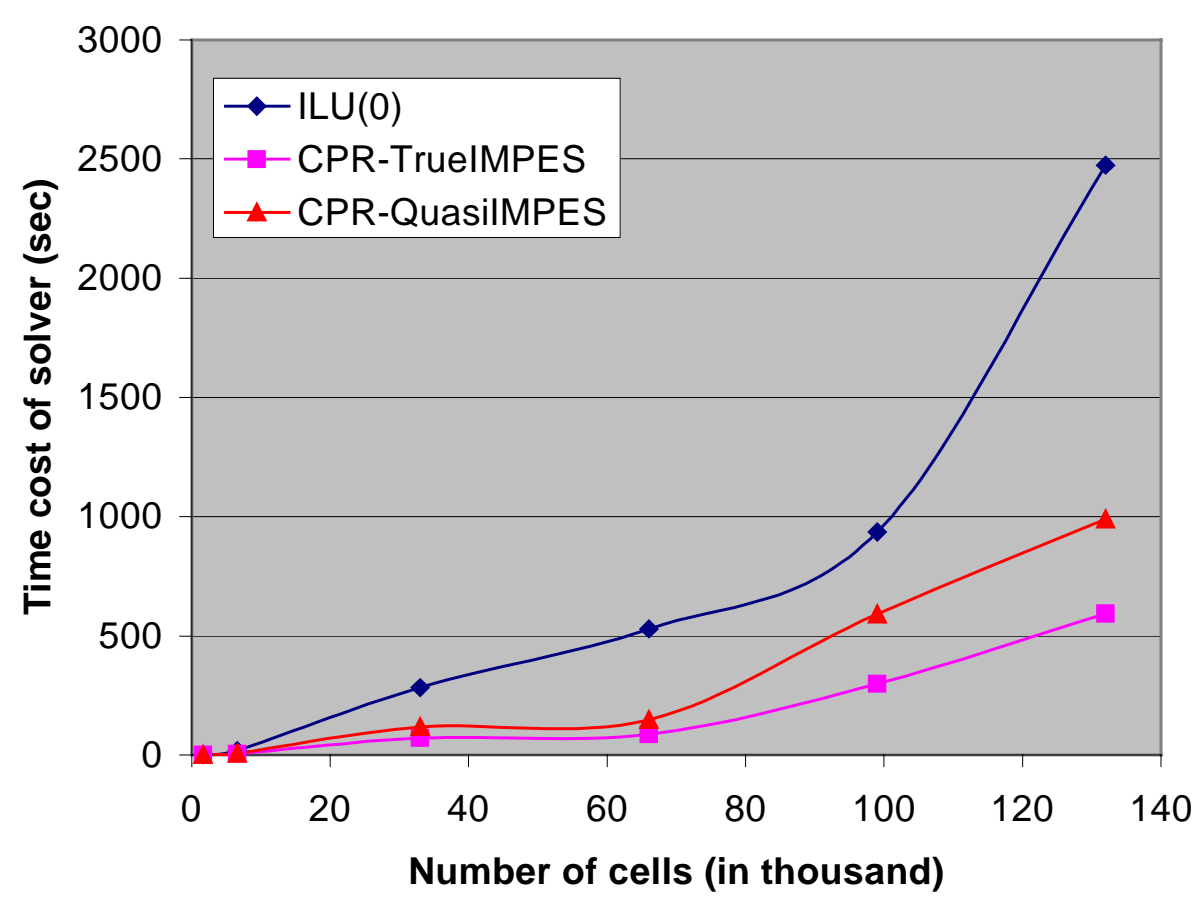

Fig. 2-13: CPU time for the linear solver with different preconditioners

From Fig. 2-13, we see that CPR with True-IMPES has the best performance. The results of Fig 2-13 are typical across a wide range of problems. The performance advantage of CPR with True-IMPES grows with problem size. 
GMRES preconditioned by CPR with True-IMPES is the best choice in GPRS. Now, we address the proper choice of the parameters of the preferred approach. Fig. 2-14 shows a flowchart for the implementation of the GMRES solver and the CPR preconditioner in GPRS.

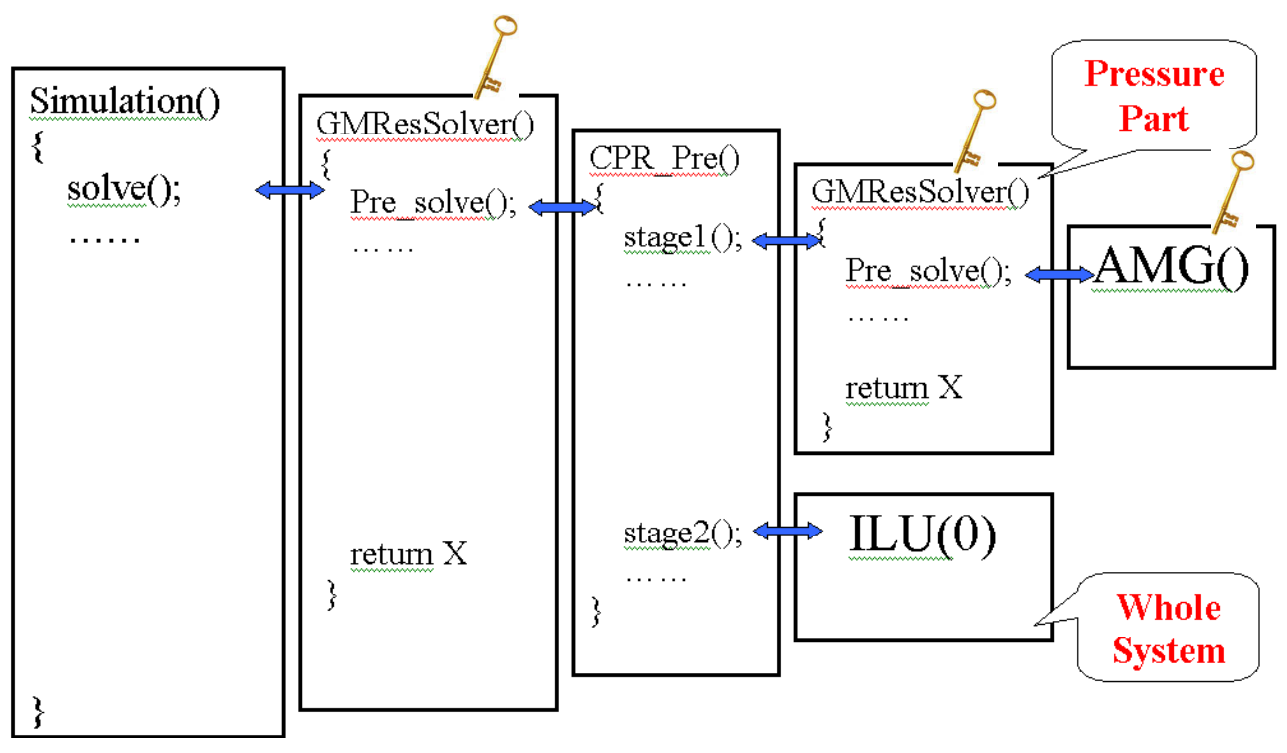

Fig. 2-14: Flowchart of simulation with GMRES solver and CPR preconditioner

In the main loop, a linear system is built for each Newton iteration. There are two stages in the CPR method. A pressure matrix is formed and extracted from the whole system in the first stage. GMRES with AMG preconditioning is used to solve this pressure system. In the second stage of CPR, ILU is applied to the entire matrix.

There are three keys in Fig. 2-14, which stand for various tolerances. These three tolerances are the most important parameters controlling the performance of the solver and preconditioner. The first key is the tolerance of the overall linear solution. Generally, this value is defined by the user. The default for this value in GPRS is 10e-6. So we will not discuss it further in this report. The second key is the tolerance of the first stage solver, i.e. the pressure solver. A reasonable value for this tolerance was determined by performing a series of simulations. For each run, we keep all the settings exactly the same, but only change 
the tolerance of the first-stage solver. Fig. 2-15 shows the relationship between the CPU time spent on the linear solver and the tolerance for a typical 3D oil-water problem.

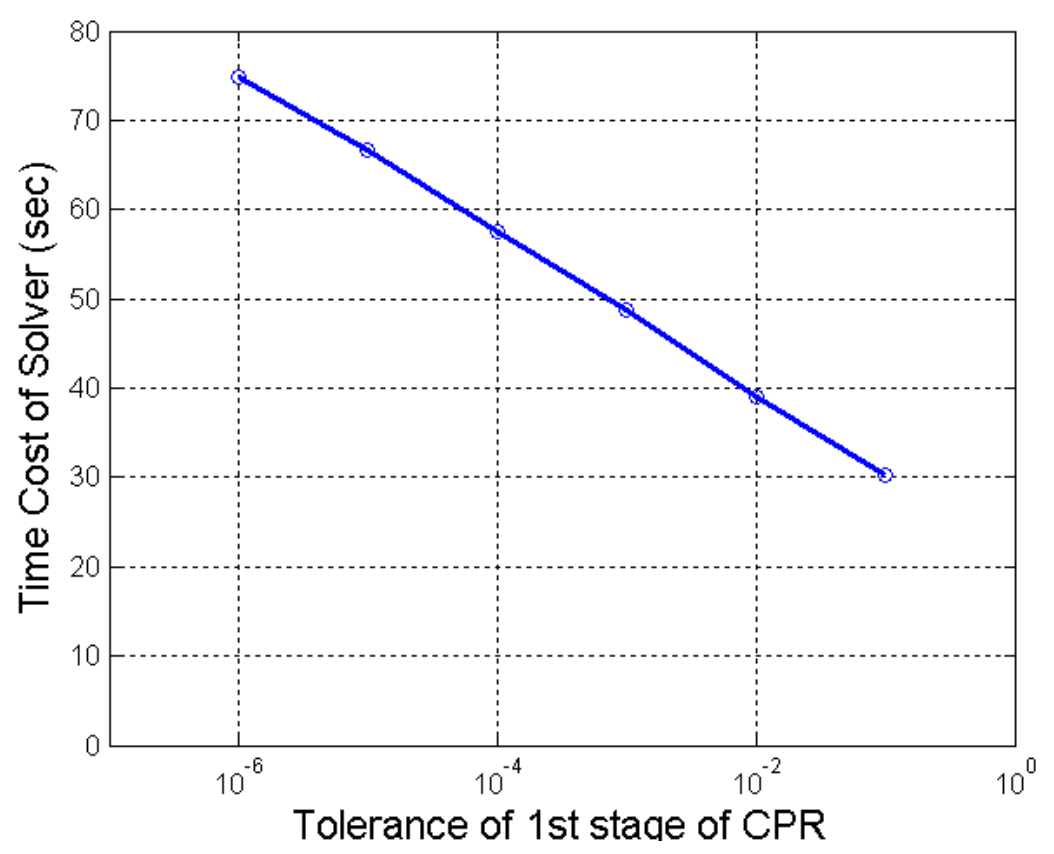

Fig. 2-15: Relation between solver time and tolerance of $1^{\text {st }}$ stage of CPR

From Fig. 2-15, we see that the time-cost appears to have a linear relation with the tolerance. Generally, we found that the tolerance of the first stage solver can be relatively loose. A reasonable choice is $10 \mathrm{e}-1$.

The third key controls the accuracy of AMG, which is the preconditioner for the pressure matrix. Basically, there are two methods to achieve this. One is to use the $l_{2}$ norm as a measure of accuracy; the other is to use a fixed number of V-cycles (Ruge and Stuben, 1987). As the number of $\mathrm{V}$-cycles increases, the solution becomes more accurate. 


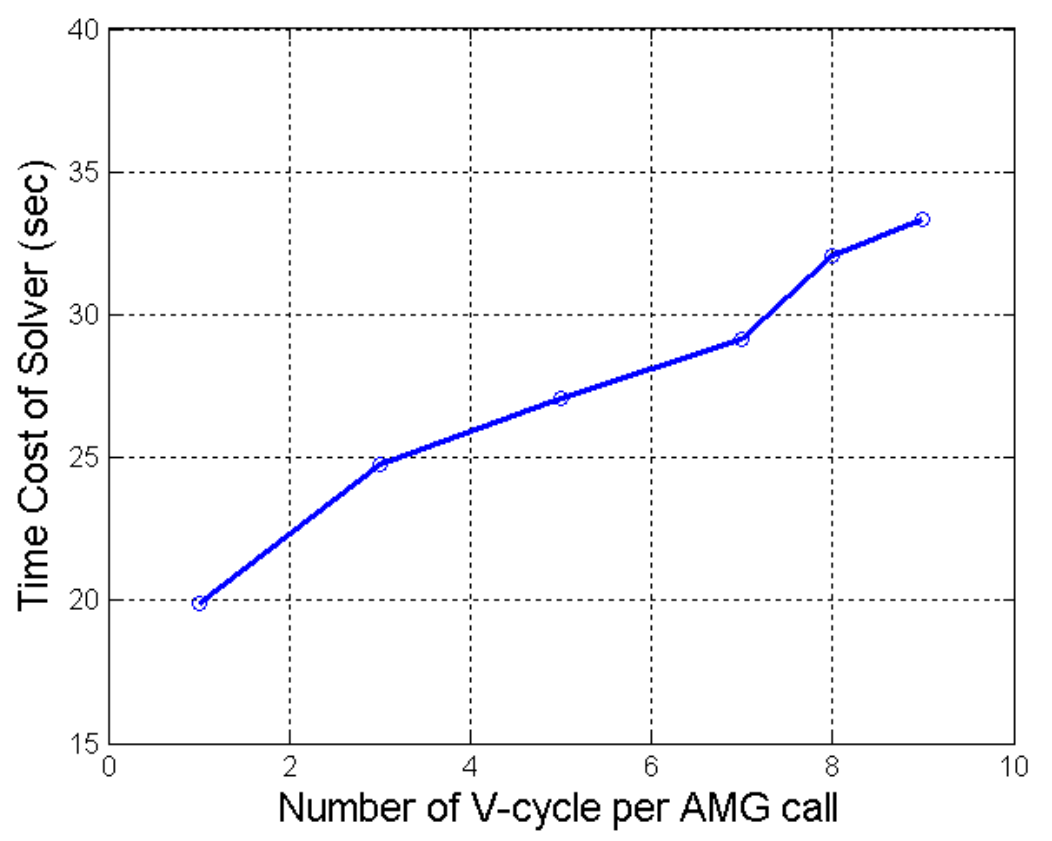

Fig. 2-16: Relation between solver time and number of V-cycle

To study the effect of the second tolerance on the overall linear solver performance, several simulations were performed where the only change was the number of V-cycles per AMG call. Fig. 2-16 shows the CPU time spent on the linear solver part versus the number of Vcycles. We notice that the cost increases when a large fixed number of V-cycles is used. So in order to achieve the best performance, we need to fix the V-cycle per AMG call to one. This finding is consistent with our wider experience, and it is the recommended choice.

\section{SAMG Solver}

SAMG is a new generation of AMG, which was developed by Stuben (2003). It has much lower CPU and memory cost. In Table 2-4, item 1 and 2 show the performance of the same problem with AMG and SAMG solvers respectively. We notice that SAMG only needs about half of the time and memory compared to that required by AMG. The test case has 141,900 cells. We expect the savings with SAMG to be even more substantial as the problem size grows. While savings in memory use in AMG are important, the overall memory consumption by the simulator in FIM mode is dominated by other factors, such as the storage of the FIM matrix as well as intermediate computations. 
Compared to the original AMG code, the new package - SAMG - provides several parameters to control the behavior of the various AMG components. For example, we can reuse the hierarchy of coarse-grid choices and interpolation operators for all Newton iterations over a timestep, and we only update (recompute) the Galerkin operators in the hierarchy (Stuben, 2003). This approach is based on the assumption that changes in the Jacobian between Newton iterations over a timestep are relatively small. The third row of Table 2-4 shows the results. This approach can lead to substantial savings, particularly for large problems.

Table 2-4: Memory and time cost of AMG and SAMG solvers

\begin{tabular}{|c|c|c|}
\hline Method & $\begin{array}{c}\text { Memory Cost of } \\
\text { solver }(\mathrm{M})\end{array}$ & $\begin{array}{c}\text { Pressure solver Time } \\
(\mathrm{sec})\end{array}$ \\
\hline AMG & 80 & 170.3 \\
\hline SAMG & 45 & 84.98 \\
\hline SAMG* & 45 & 70.94 \\
\hline
\end{tabular}

\section{Applications}

SPE10 is described on a Cartesian grid, with 1,122,000 cells (60x220x85), with a highly heterogeneous permeability field. The dimensions of SPE10 are 1200x2200x170 (ft). "The top $70 \mathrm{ft}$ (35 layers) represents the Tarbert formation, and the bottom $100 \mathrm{ft}$ (50 layers) represents Upper Ness" (Christie and Blunt, 2001). SPE10 is widely used in research work, especially as a challenging test problem.

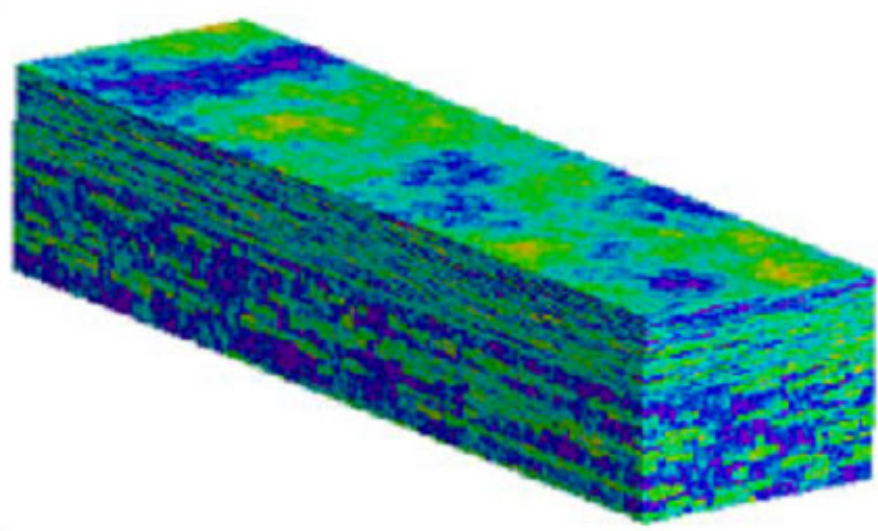

Fig. 2-17: Porosity map of SPE10 (Christie et al., 2001) 
In order to validate the performance of the optimized solver and preconditioners, we ran the same cases using the Eclipse simulator (GeoQuest, Schlumberger, 2001) and GPRS in the same computing environment. The testing platform is an NTTS server, which has four $2.8 \mathrm{G}$ Hz CPUs, 4G memory. The operating system is Microsoft Windows 2000, Server package 4, which support up to $2 \mathrm{G}$ memory for one program. Since the current version of GPRS does not support parallel computing, we do not get much gain from the multi-CPU system.

\section{Case 1: Upscaled (1/8) SPE10}

In the first case, we upscaled the SPE10 problem in each of the three coordinate directions by a factor of two. The grid is $110 \times 30 \times 43$ (141,900 cells). An injection well is located at the corner of the reservoir with water rate control and a production well is in the middle of reservoir using BHP control. Both wells fully penetrate the 43 layers.

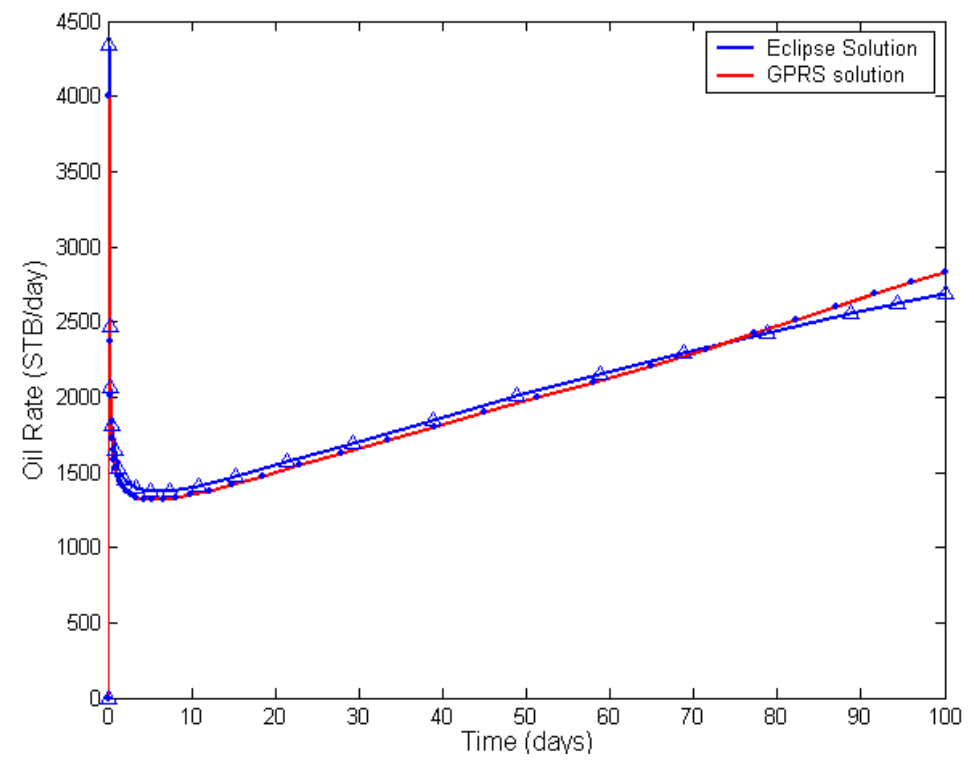

Fig. 2-18: Oil rate curves of Eclipse and GPRS with an upscaled SPE10 problem 
Table 2-5: Performances of GPRS and Eclipse with upscaled SPE10 case

\begin{tabular}{|c|c|c|}
\hline & GPRS & Eclipse \\
\hline Pressure Solver Time $(\mathrm{sec})$ & $371(39 \%)$ & - \\
\hline Linear Solver Time $(\mathrm{sec})$ & $802(84 \%)$ & - \\
\hline Total Time $(\mathrm{sec})$ & $956(100 \%)$ & 5160 \\
\hline Time Step & 19 & 23 \\
\hline Newton Iteration & 102 & 88 \\
\hline Newton Iteration / Time Step & 5.4 & 3.8 \\
\hline Linear Iteration & 574 & 5515 \\
\hline Pressure Iteration & 815 & - \\
\hline
\end{tabular}

The oil production rate obtained from GPRS and the Eclipse simulator are shown in Fig. 218. The match between the two simulators is very good. The detailed performance of the two simulators is shown in Table 2-5. The time cost of GPRS is only about $20 \%$ of the Eclipse simulator's time cost. There is no significant difference in the number of timesteps, but GPRS took more Newton iterations. However, the numbers of linear iteration for the two simulators are very different. The Eclipse simulator uses Orthomin preconditioned by nested factorization (GeoQuest, Schlumberger, 2001).

We can see that the linear solver in GPRS is quite efficient for this case. This is primarily due to the use of the CPR approach, with AMG as a preconditioner for the first stage.

\section{Case 2: Bottom 50 layers of SPE10}

Due to memory constraints, we can only run simulations with up to 50 layers of the original SPE10 model. We selected the bottom 50 layers of SPE10 as our model, which is the more difficult part of SPE10. Fig. 2-19 shows the oil rate for this case obtaned using GPRS. In this case, the Eclipse simulator did not converge. 


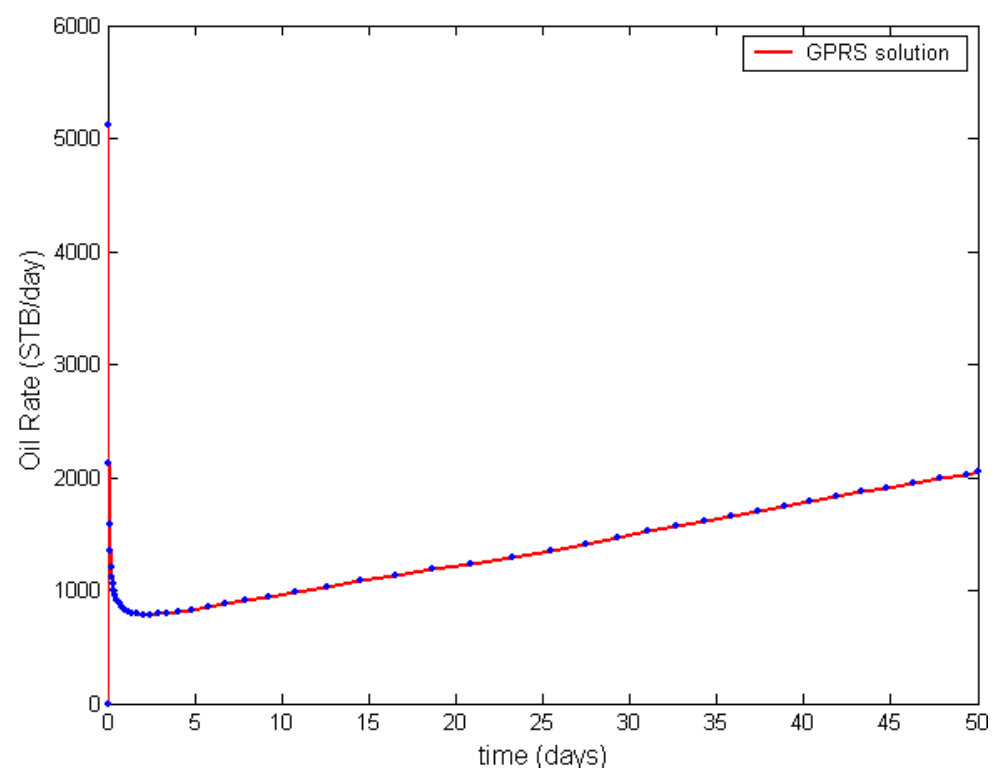

Fig. 2-19: Oil rate curve of GPRS in bottom 50 layers of SPE10

Table 2-6: Performances of GPRS and Eclipse with bottom 50 layers of SPE10

\begin{tabular}{|c|c|}
\hline & GPRS \\
\hline Pressure Solver Time (sec) & $4160(41 \%)$ \\
\hline Linear Solver Time $(\mathrm{sec})$ & $8724(86 \%)$ \\
\hline Total Time $(\mathrm{sec})$ & $10156(100 \%)$ \\
\hline Time Step & 50 \\
\hline Newton Iteration & 195 \\
\hline Newton Iteration / Time Step & 3.9 \\
\hline Linear Iteration & 1342 \\
\hline Pressure Iteration & 2076 \\
\hline
\end{tabular}

We note here that default parameter settings were used for Eclipse, and no attempt was made to optimize the various parameters for the linear and nonlinear solvers. We only tried increasing the maximum number of linear solver iterations because the simulator prompted us to do so. For GPRS, we used 1.e-6 as the tolerance for the linear solver.

GPRS is not as efficient in memory use as the Eclipse simulator. For example, for Case 2, GPRS needs about 1.8 Giga bytes; while the Eclipse simulator needs only about 0.6 Giga bytes. In order to make the code clear and easier to follow, GPRS stores the same matrix in two different ways, which is one reason for the excessive memory use. GPRS also forms a separate pressure matrix for the first stage. In addition to the basic variables, such as 
saturations and porosity, GPRS stores many intermediate results, such as the mobility and its various derivatives, in order to save computing time. These are some of the reasons for excessive memory use in GPRS.

\subsection{Implementation of the Tracer Option}

Modeling of tracer flow using TVD schemes is now possible in GPRS. Since we assume that the tracer does not affect the properties of the carrier fluid, the tracer concentration term does not appear in the multiphase flow equations. Therefore, the problem of tracer flow is naturally decoupled from the multiphase flow problem. We can solve the multiphase flow equations first, and then update the concentration in an explicit step using the dual timestepping technique. This also makes the tracer option a relatively isolated module in the GPRS code, which is very good from the viewpoint of developing and maintaining the software. Full details are available in the M.S. report of Jiang (2004).

\section{Validation of Tracer Option}

In this section, we will compare the three TVD limiters (Jiang 2004) and validate our implementation of the tracer option.

First we consider a one-dimensional two-phase oil-water system with a connate water saturation of 0.15 . This system is homogeneous with $25 \%$ porosity and 500 md permeability. The number of cells is 50. One injection well is located in the middle of the first grid block, and one production well is in the last grid block. The water with tracer is injected from the injection well.

As mentioned before, the carrier-phase saturation has an important effect on the tracer concentration, so we always plot the water saturation and tracer concentration curves. Our results are also compared with the tracer results obtained from the industry-standard Eclipse simulator (GeoQuest Schlumberger, 2001). Fig. 2-20, 2-21 and 2-22 show results for this problem with the three limiters discussed previously when PVI is equal to 0.28. 


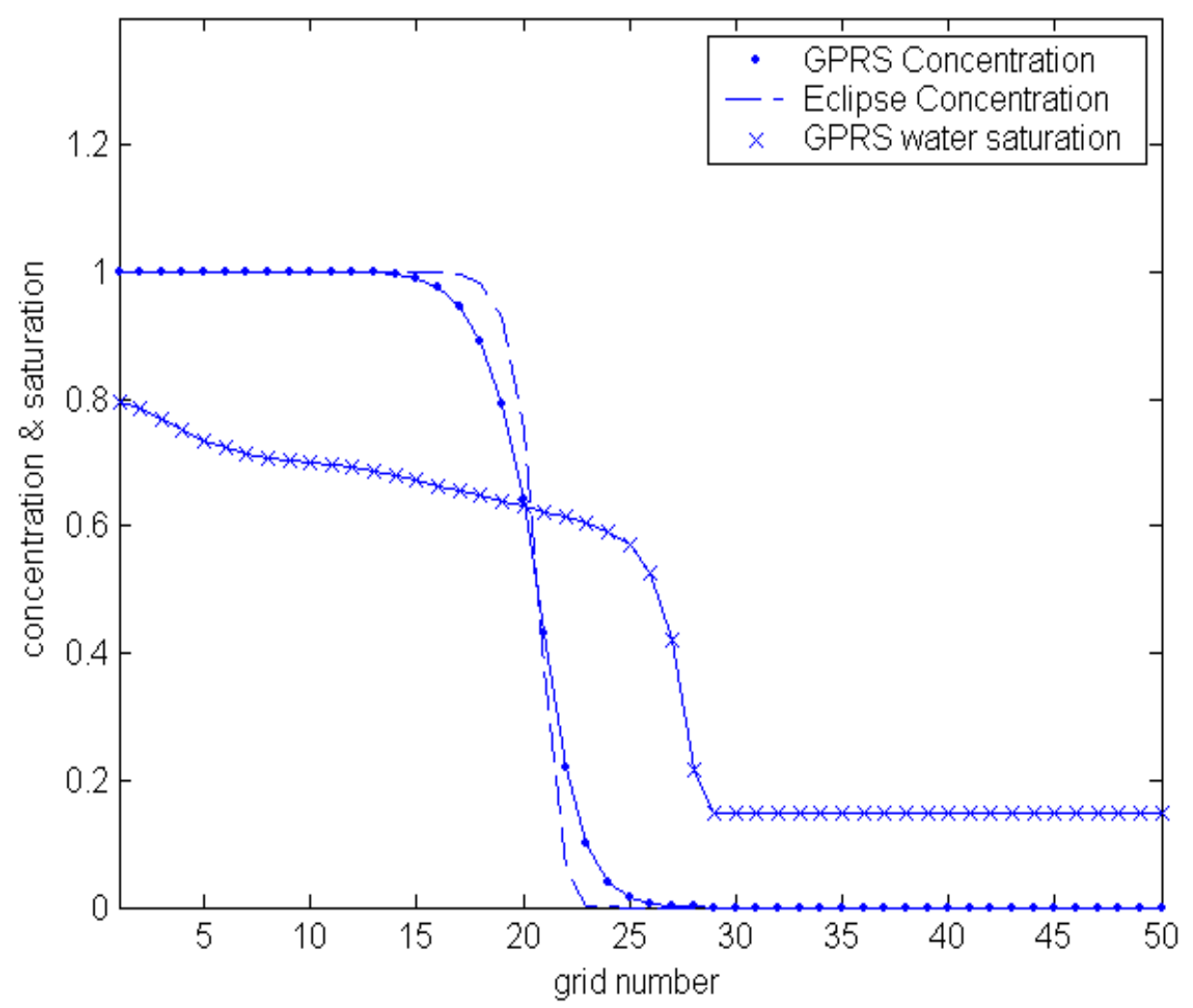

Fig. 2-20: Result of Eclipse and result of GPRS with the Minmod limiter

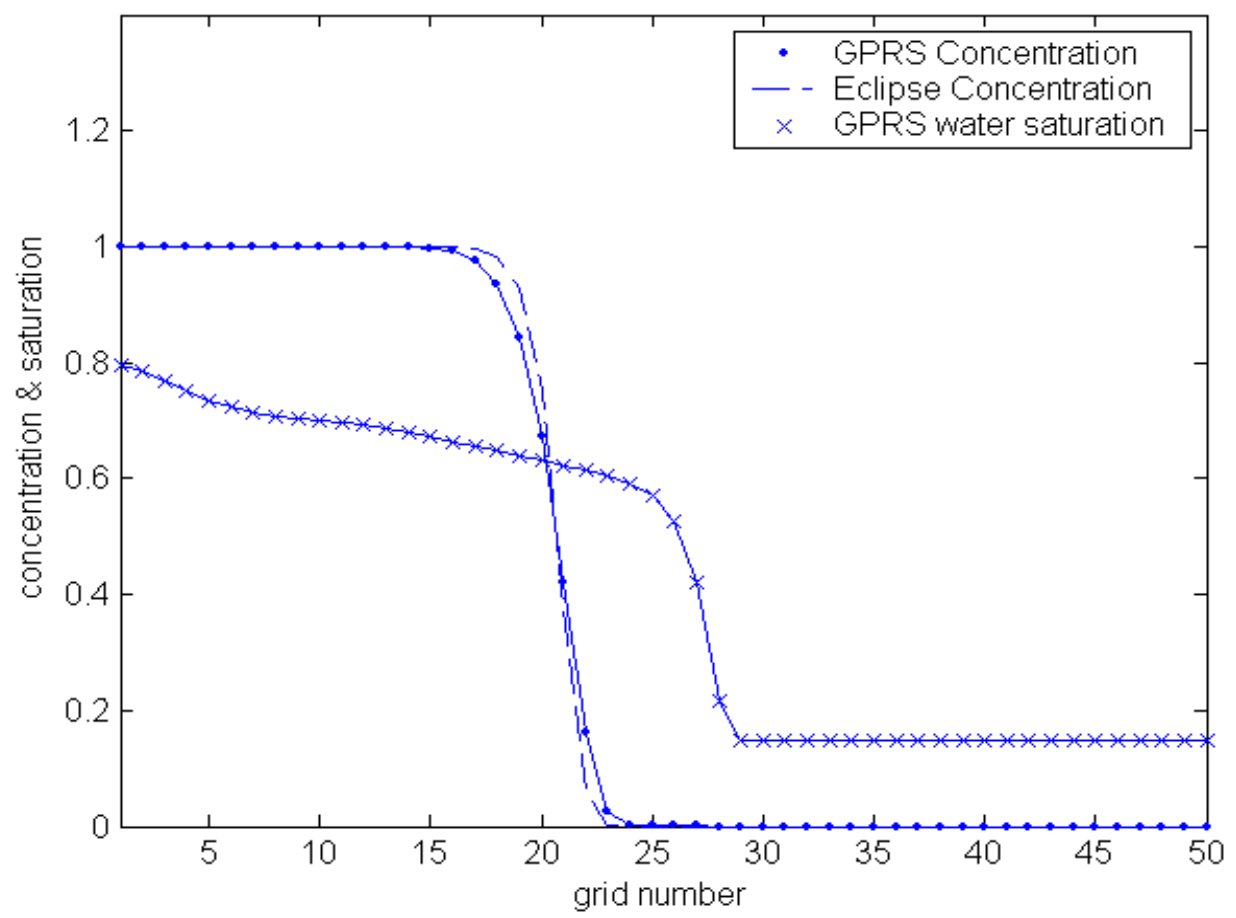

Fig. 2-21: Result of Eclipse and result of GPRS with the Van Leer limiter 


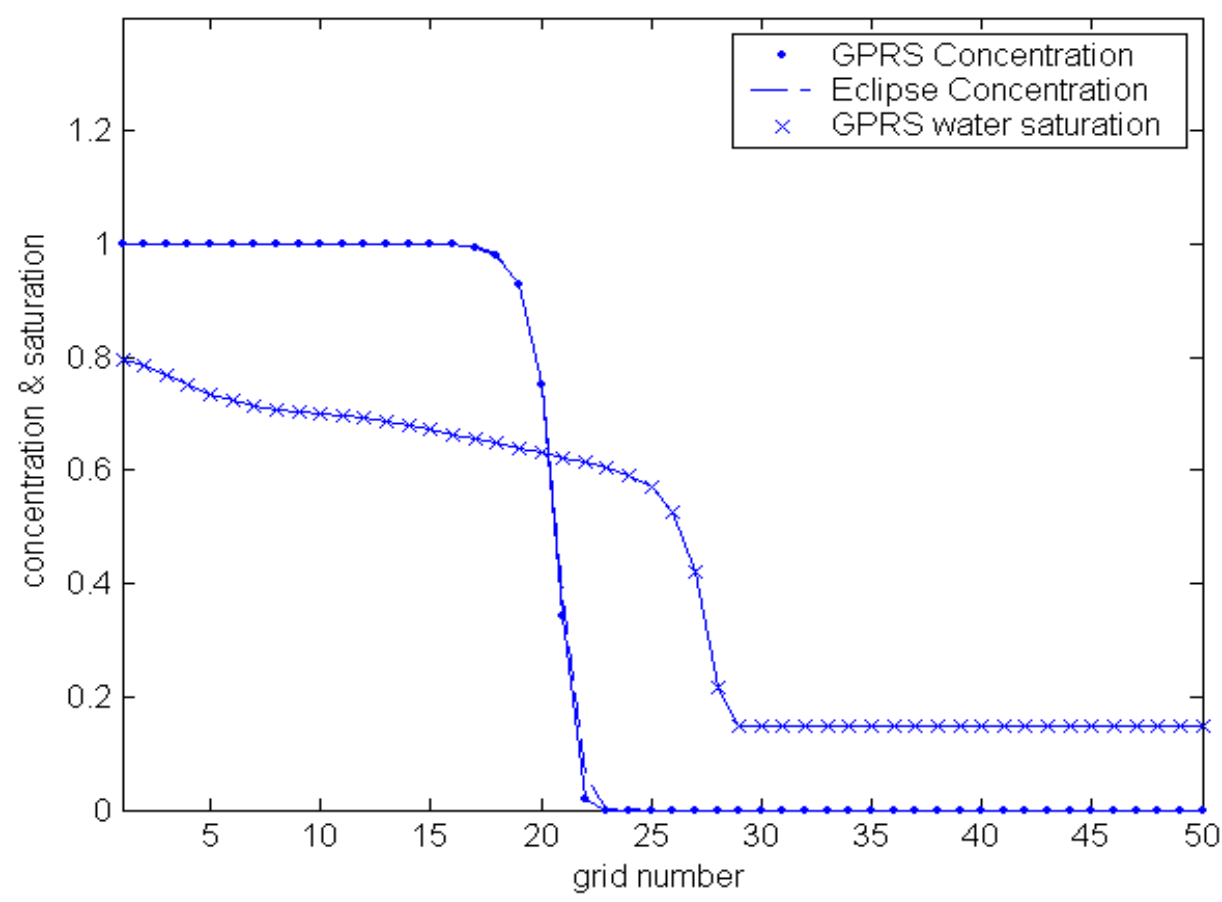

Fig. 2-22: Result of Eclipse and result of GPRS with the Superbee limiter

We can see that the Superbee limiter (Fig. 2-22) has the best anti-diffusion properties and produces results very close to those from the Eclipse simulator. The anti-diffusion properties of the Minmod limiter are not as good as those of the other two, but the TVD scheme with the Minmod limiter still performs much better than the upstream scheme (Fig. 2-20).

To further test the tracer implementation in GPRS, a slug injection case is considered. The system setting is the same as that in the previous case, except that the tracer injection is stopped after 1000 days to form a concentration slug (PVI is equal to 0.056). Note that water was injected continuously. Because the slug is very sensitive to numerical diffusion, this is a good case to illuminate the difference in the anti-diffusion properties of different limiters. Another goal of this test case is to make sure that the scheme is valid when the extreme point is in the middle of the domain rather than on the boundary. Since the basic implementation has been verified in the previous case, we do not provide the results of the Eclipse simulator in this case. Fig. 2-23 and 2-24 show the results with the application of Minmod and Superbee limiters after 5000 days injection (PVI is equal to 0.28). Again, the Superbee limiter produces the best results. 


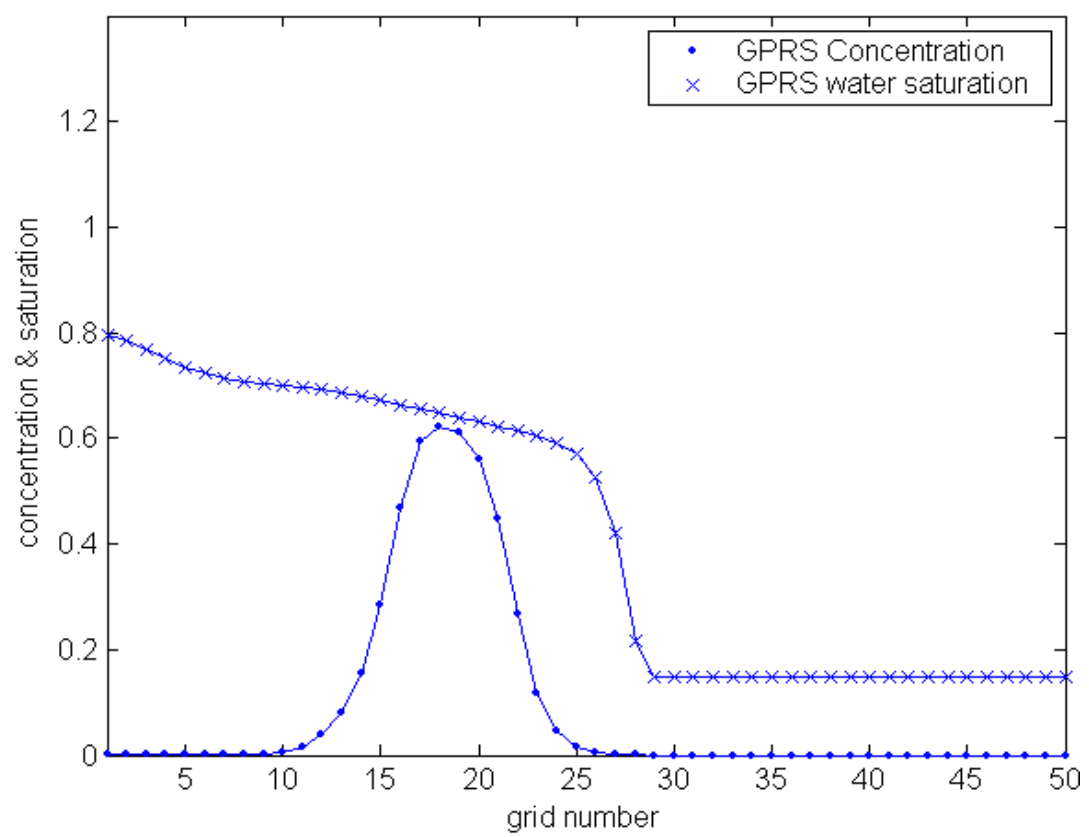

Fig. 2-23: Slug solution with Minmod limiter in GPRS

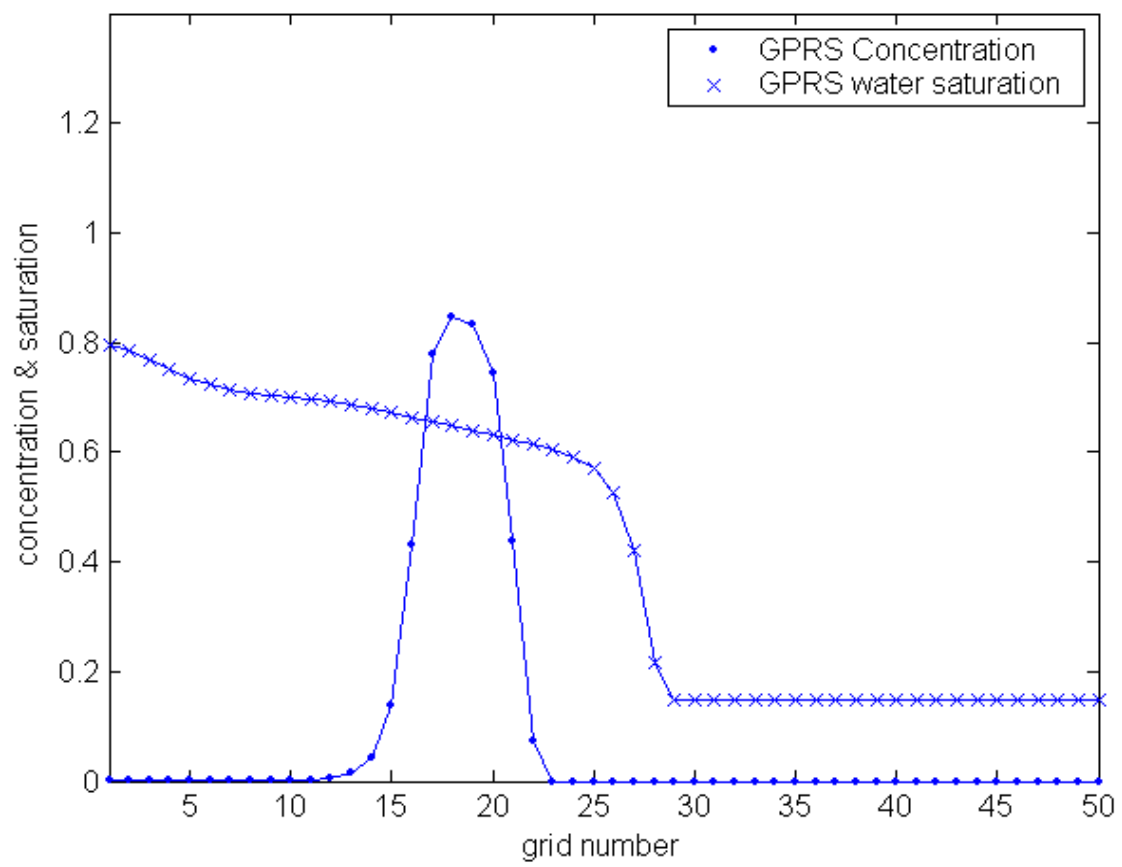

Fig. 2-24: Slug solution with Superbee limiter in GPRS 
Since the Superbee limiter has the best performance, we use it in the further testing of the tracer option in GPRS. The TVD model with the Superbee limiter was extended to 2D and $3 \mathrm{D}$.

\section{Extension of TVD to 2D and 3D and More Validations}

The TVD scheme is extended to 2D and 3D by applying the 1D scheme in each direction. It is worth mentioning two points here. One, there is no theoretical proof of the TVD property of models other than in 1D. However, numerous numerical works show that TVD schemes are valid in 2D and 3D model (Fu, 1993). The other point is we did not account for the mixed derivative term. Therefore, the results of the scheme may not be strictly of secondorder accuracy. In order to validate the implementation of the tracer option, more test cases have been performed with GPRS, and the results are compared with results from the Eclipse simulator.

\section{Case 1: Homogeneous 2D test case}

In this case, we have an oil-water 2D homogeneous system. The connate water saturation is 0.15 . One water injection well is located at the center of the reservoir, and one production well is at the upper right corner. The tracer is injected with water from the injection well. After 1000 days of running ( 0.075 PVI), the concentration maps of GPRS and the Eclipse simulator are shown in Fig. 2-25. 

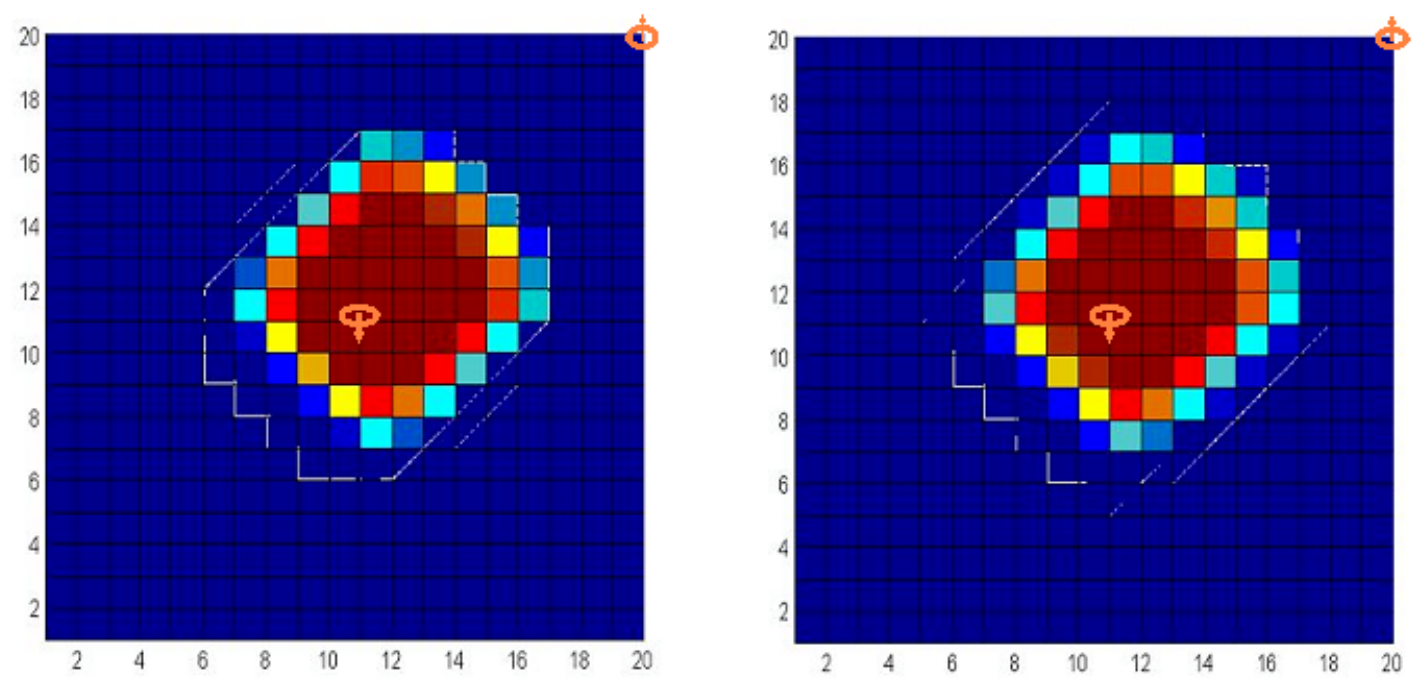

Fig. 2-25: Tracer concentration maps from GPRS (left) and Eclipse (right) results with injection at the center

The results from the two simulators match well. In order to compare the results more accurately, a cross-sectional view is provided in Fig. 2-26.

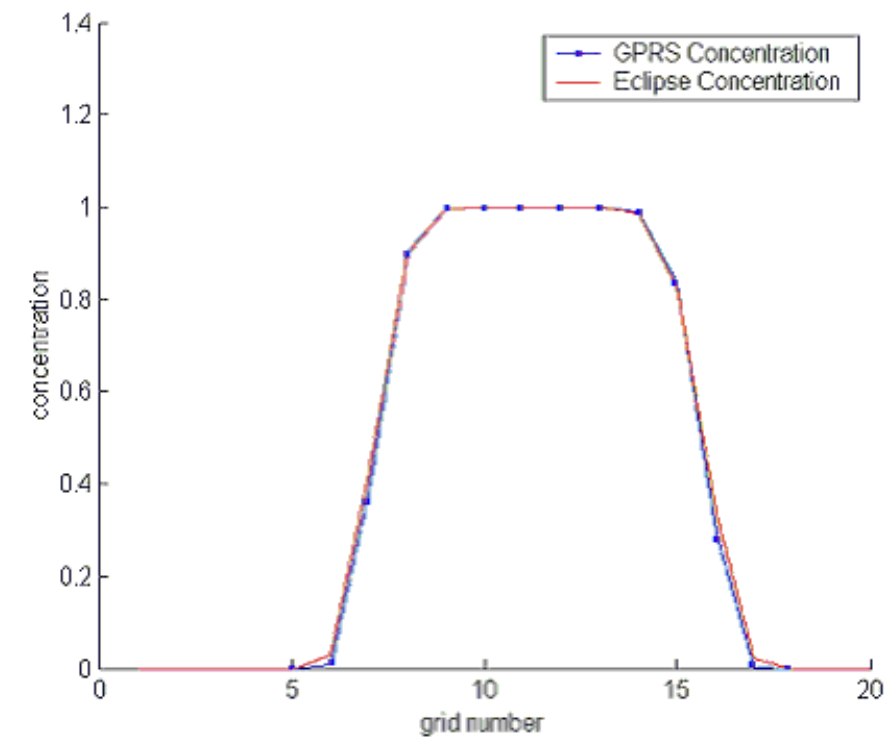

Fig. 2-26: Cross-sectional view of the concentrations at the $11^{\text {th }}$ column

The cross-sectional view is given from the $11^{\text {th }}$ column, in which the injection well is located. Basically, the match is very good. 


\section{Case 2: Heterogeneous 2D test case}

In this case, the permeability map contains four stripes with two permeability values: $100 \mathrm{md}$ and 1000 md, as shown in Fig. 2-27. The injection well is located at the left lower corner, and the production well is at the upper right corner. The TVD scheme results of GPRS and the Eclipse simulator are shown in Fig. 2-27. The GPRS upstream results are also provided.
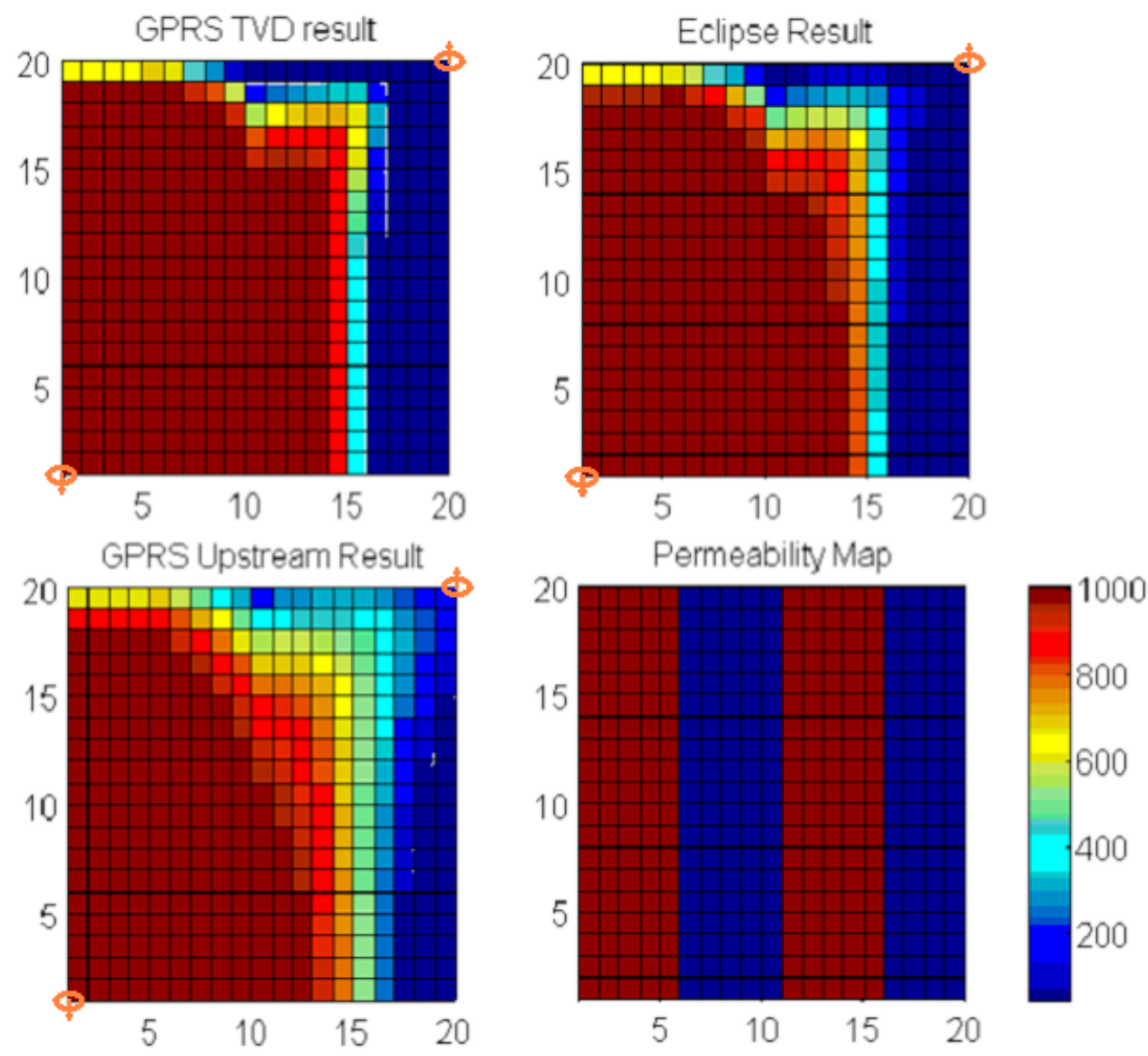

Fig. 2-27: Tracer concentration maps of GPRS and Eclipse and the permeability field

TVD results from GPRS are very similar to the results from the Eclipse simulator and both of them are much better than the upstream results. A cross-sectional view given from the $15^{\text {th }}$ row provides more details about the results, as shown in Fig. 2-28. 


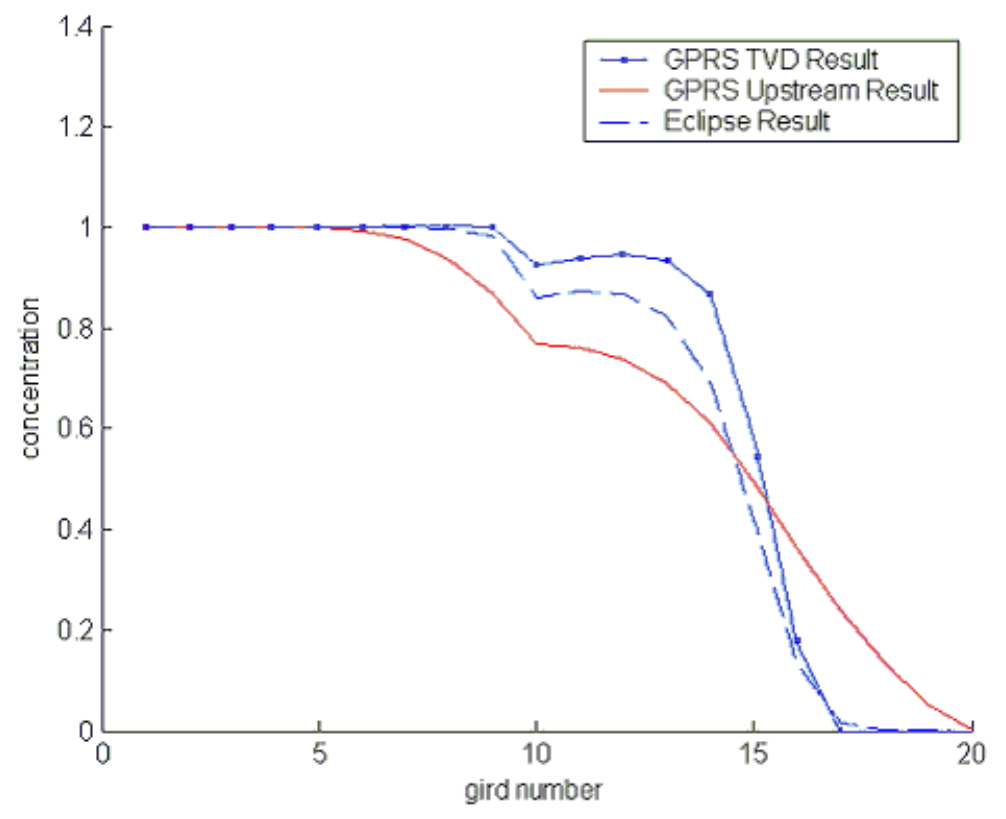

Fig. 2-28: Cross-sectional view of tracer concentration at the $15^{\text {th }}$ row

The TVD TVD scheme in GPRS gives the sharpest profile. From the plots in this figure, it may seem that there is some "mass balance error" among these three results. But, remember that Fig. 2-28 only shows a slice of the whole 2D problem. We do not have any significant overall mass balance errors in the tracer simulation. The tracer distributions are different, due to the different anti-diffusion properties of the various schemes.

\section{Case 3: Homogeneous 3D test case}

In this case, we have an oil-water 3D homogeneous system with $20 \times 20 \times 3$ grid. The connate water saturation is 0.15 . One water injection well is located at the left lower corner of the system, and it is perforated in the top layer; the production well is at the upper right corner of the system, and it is penetrated in the bottom layer. Tracer is injected with water from the injection well. After a few days running (PVI is equal to 0.3), the concentration maps of GPRS and Eclipse are shown in Fig. 2-29. 

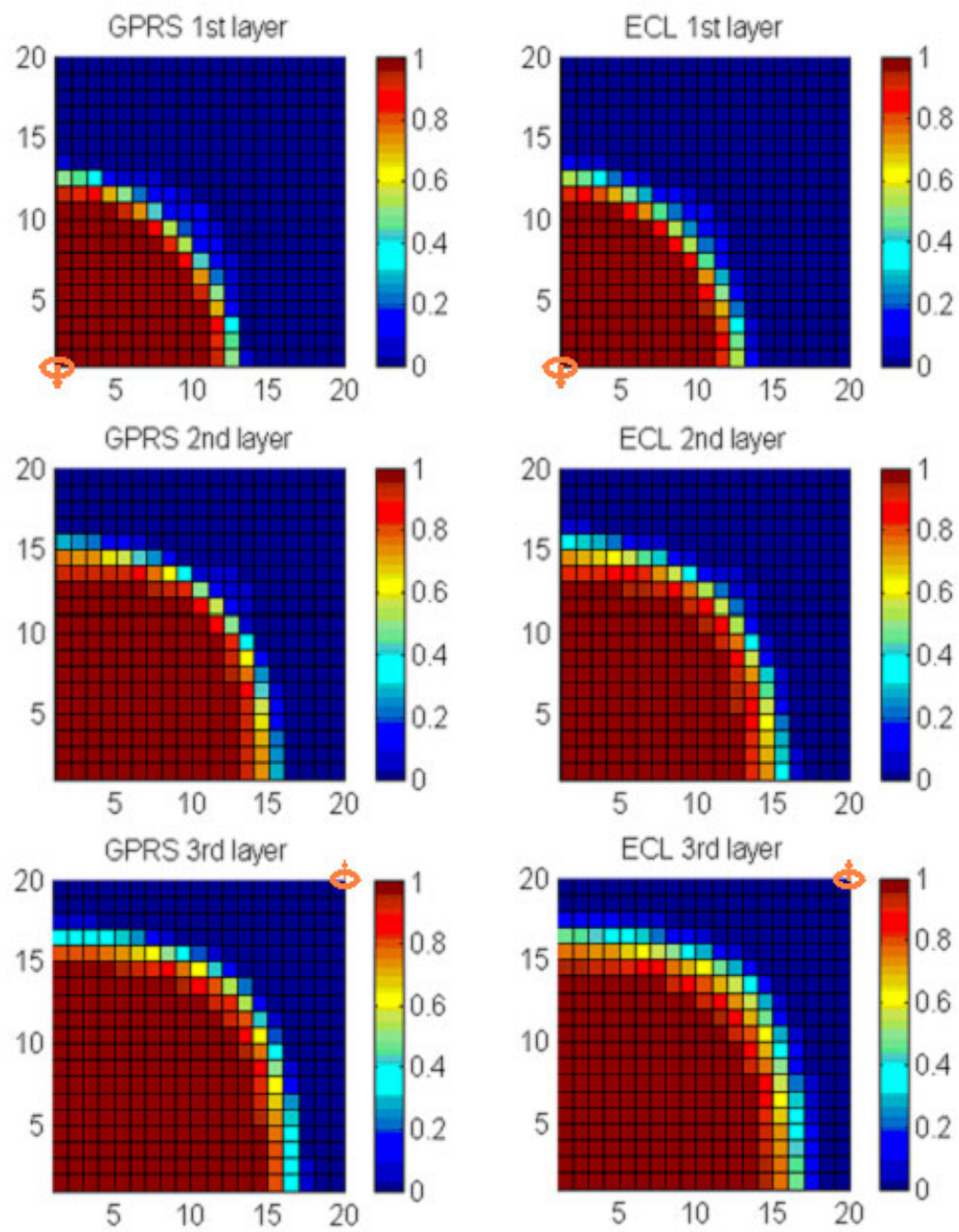

Fig. 2-29: Tracer concentration map of GPRS (left) and Eclipse (right) results

Fig. 2-30 shows the GPRS and Eclipse tracer distribution in the $1^{\text {st }}$ row of the bottom layer. The two results are very similar, and the one from GPRS is a little sharper than the one from Eclipse.

From the test cases discussed above, and a variety of additional cases, we can conclude that the tracer option in GPRS in 2D and 3D is robust, and that the GPRS results tend to yield tracer fronts with slightly lower levels of numerical dispersion. 


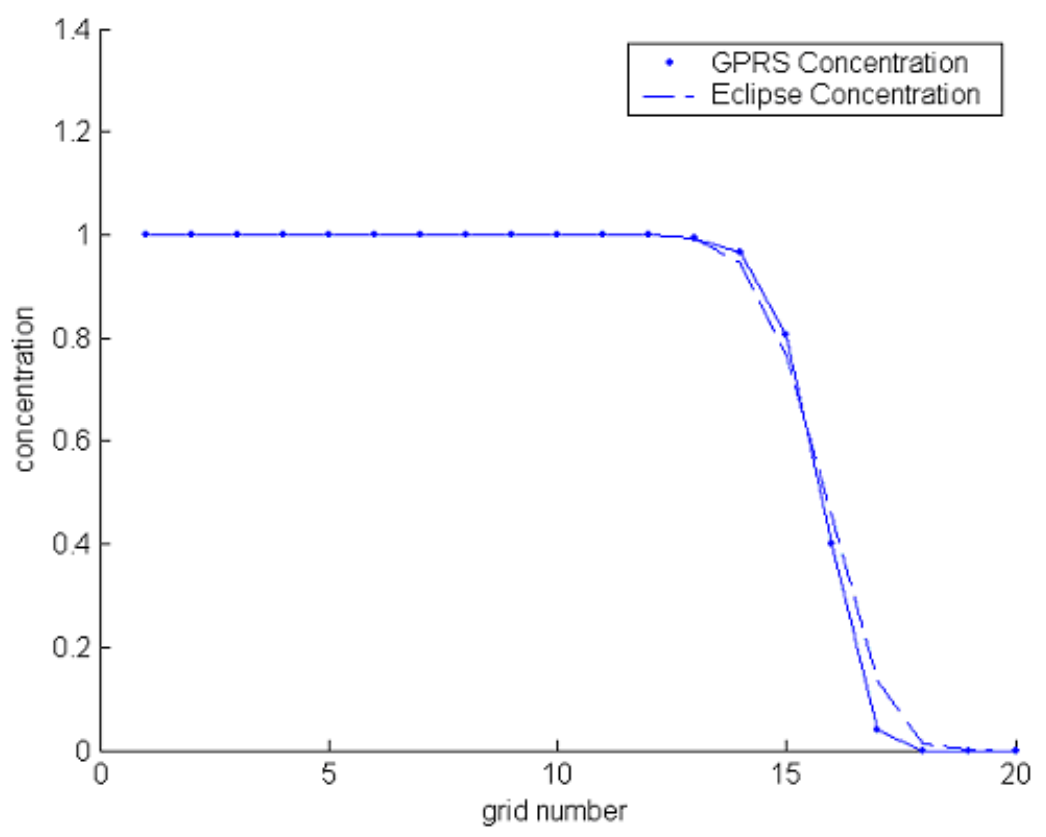

Fig. 2-30: Cross-sectional view of tracer concentration at the $1^{\text {st }}$ row

\section{Nomenclature}
$A=\quad$ Coefficient matrix of the linear system
$A_{p} \quad=\quad$ IMPES pressure matrix
$A_{p}{ }^{d}=\quad$ Diagonal term of the pressure matrix
$A_{p}{ }^{o} \quad=\quad$ Off-diagonal term of the pressure matrix
$A_{p}{ }^{w}=\quad$ Well term of the pressure matrix
$A_{p p}=\quad$ Derivative of accumulation term in primary equation wrt. Pressure
$A_{p s}=\quad$ Derivative of accumulation term in primary equation wrt. Saturation
$A_{s p}=\quad$ Derivative of accumulation term in secondary equation wrt. Pressure
$A_{s s}=$ Derivative of accumulation term in secondary equation wrt. Saturation
$A_{r r} \quad=\quad$ Sub-matrix whose elements are the derivatives of reservoir equations wrt. reservoir variables 


\begin{tabular}{|c|c|c|}
\hline$A_{r w}$ & $=$ & Sub-matrix whose elements are the derivatives of reservoir equations wrt. well variables \\
\hline$A_{w r}$ & $=$ & Sub-matrix whose elements are the derivatives of well equations wrt. reservoir variables \\
\hline$A_{w w}$ & $=$ & Sub-matrix whose elements are for the derivatives of well equations wrt. well variables \\
\hline$A_{r r}^{*}$ & $=$ & Schur complement \\
\hline$b_{o}$ & $=$ & Reciprocals of the formation volume factor (FVF) of oil phase. \\
\hline$b_{w}$ & $=$ & Reciprocals of the formation volume factor (FVF) of water phase \\
\hline$c_{i}$ & $=$ & Tracer concentration in cell $i$ \\
\hline$c_{i n}$ & $=$ & Upstream tracer concentration of two cells \\
\hline$C$ & $=$ & Column operation matrix \\
\hline$C_{o}$ & $=$ & Water phase compressibility \\
\hline$C_{w}$ & $=$ & Oil phase compressibility \\
\hline$\vec{e}$ & $=$ & Vector with all ' 1 ' elements \\
\hline$f$ & $=$ & Flux \\
\hline$f(u)$ & $=$ & Flux of $u$. \\
\hline$f_{w}$ & $=$ & Water flux \\
\hline$F_{i \rightarrow l}$ & $=$ & Molar flow rate of the carrier phase from cell $i$ to neighboring cell $l$ \\
\hline$F_{p p}$ & $=$ & Derivative of flux term in primary equation wrt. Pressure \\
\hline$F_{p s}$ & $=$ & Derivative of flux term in primary equation wrt. Saturation \\
\hline$F_{s p}$ & $=$ & Derivative of flux term in primary equation wrt. Pressure \\
\hline$F_{s s}$ & $=$ & Derivative of flux term in secondary equation wrt. Saturation \\
\hline$F_{p p}^{o}$ & $=$ & Derivative of flux term in primary equation wrt. neighbor cell pressure \\
\hline$F_{p s}^{o}$ & $=$ & Derivative of flux term in primary equation wrt. neighbor cell saturation \\
\hline$F_{s p}^{o}$ & $=$ & Derivative of flux term in secondary equation wrt. neighbor cell pressure \\
\hline$F_{s s}^{o}$ & $=$ & Derivative of flux term in secondary equation wrt. neighbor cell saturation \\
\hline$F_{p p}{ }^{w}$ & $=$ & Derivative of well flux term in primary equation wrt. neighbor cell pressure \\
\hline$F_{s p}^{w}$ & $=$ & $\begin{array}{l}\text { Derivative of well flux term in secondary equation wrt. neighbor cell } \\
\text { pressure }\end{array}$ \\
\hline
\end{tabular}




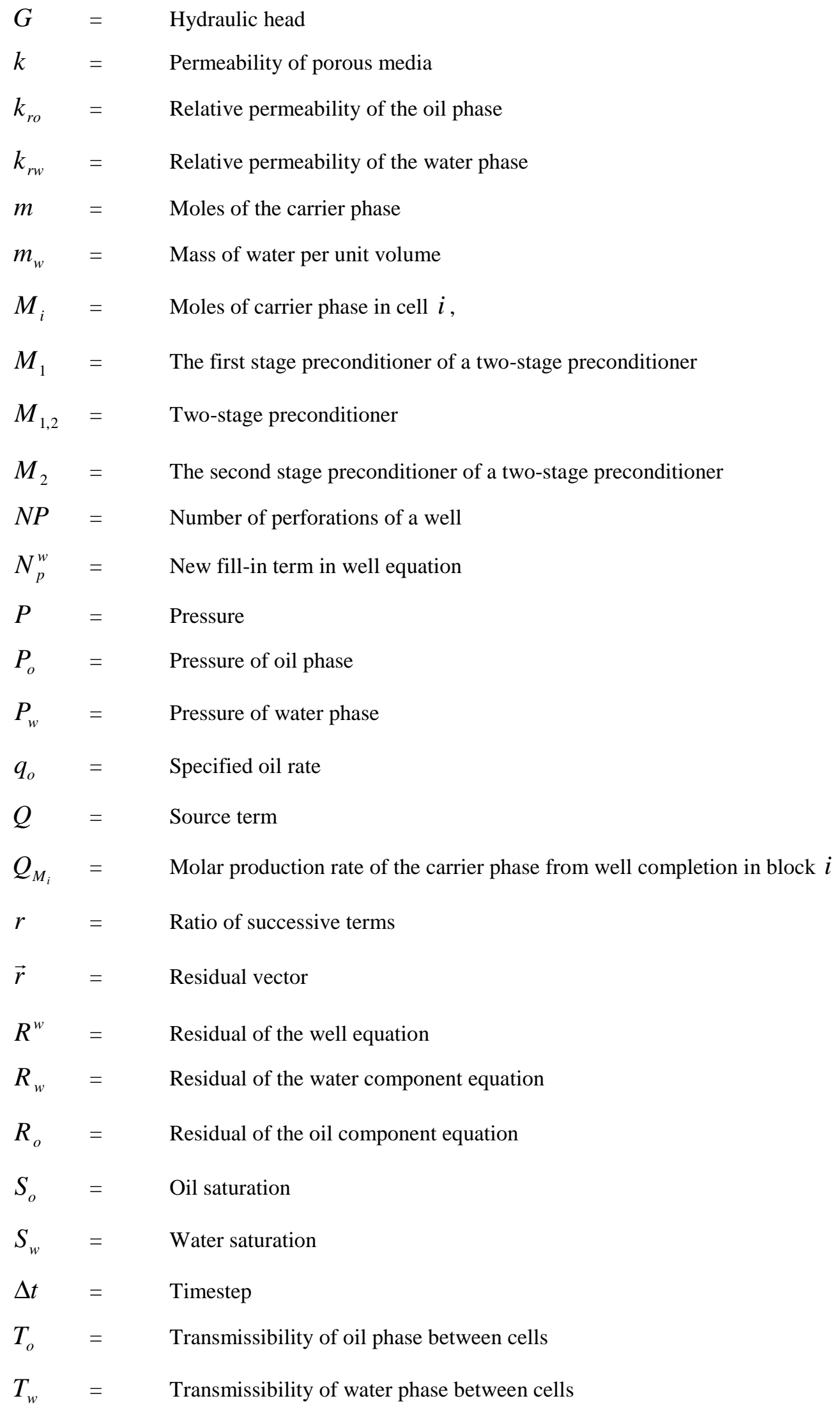




$$
\begin{array}{lll}
T_{T}^{w} & = & \text { Total well transmissibility } \\
T_{o}^{w} & = & \text { Well transmissibility of oil phase } \\
T_{w}^{w} & = & \text { Well transmissibility of water phase } \\
\vec{u} & = & \text { Solution vector } \\
V_{i} & = & \text { The bulk volume of cell } i \\
W^{\mathrm{T}} & = & \text { Row operation matrix } \\
W I & = & \text { Well index }
\end{array}
$$

\section{Acronyms}

$\begin{array}{lll}\mathrm{AIM}= & \text { Adaptive implicit method } \\ \mathrm{AMG}= & \text { Algebraic multi-grid } \\ \mathrm{BHP}= & \text { Bottom hole pressure } \\ \mathrm{CPR}= & \text { Constrained pressure residual } \\ \mathrm{FIM}= & \text { Fully implicit method } \\ \mathrm{FVF}= & \text { Formation volume factor } \\ \mathrm{GMRES}= & \text { Generalized minimum residual } \\ \text { GPRS }= & \text { General Purpose Research Simulator } \\ \mathrm{ILU}= & \text { Incomplete LU decomposition } \\ \mathrm{IMPES}= & \text { Implicit pressure and explicit saturations } \\ \mathrm{IMPSAT}= & \text { Implicit pressure and saturations } \\ \mathrm{PVI}= & \text { Pore Volume Injected } \\ \mathrm{TVD}= & \text { Total variance diminishing }\end{array}$

\section{Greek}
$\Delta=$ Change
$\rho_{o}=\quad$ Density of oil phase
$\rho_{w}=\quad$ Density of water phase
$\mu_{o} \quad=\quad$ Viscosity of oil phase
$\mu_{w} \quad=\quad$ Viscosity of water phase
$\phi \quad=\quad$ Porosity
$\varphi(r)=\quad$ Limiter function 


\section{Subscripts}

$\begin{array}{lll}p & = & \text { Pressure } \\ P & = & \text { Pressure } \\ o & = & \text { Oil } \\ s & = & \text { Saturation } \\ S & = & \text { Saturation } \\ w & = & \text { Water }\end{array}$

\section{Superscripts}

$\begin{array}{lll}d & = & \text { Diagonal } \\ w & = & \text { Well } \\ o & = & \text { Off-diagonal } \\ n & = & \text { Time level } n \\ n+1 & = & \text { Time level } n+1\end{array}$




\section{References}

Ali, E., Chatzichristos, C., Aurdal, T. and Muller, J. "Tracer Simulation to Improve the Reservoir Model in the Snorre Field”. SPE 64796, SPE International Oil and Gas Conference and Exhibition in China held in Beijing, China, November 7-10, 2000

Anderson, E. LAPACK (Linear Algebra Package), www.netlib.org 1999

Aziz, K, Durlofsky, L. J. and Gerritsen, M. "Notes for Petroleum Reservoir Simulation”. Stanford University, Winter 2003

Aziz, K. and Settari, A. "Petroleum Reservoir Simulation". Applied Science Publishers, London, 1979

Behie, G. A. and Forsyth, P. A. "Incomplete Factorization Methods for Fully Implicit Simulation of Enhanced Oil Recovery”. SIAM J. Sci. Stat. Comp. Vol. 5, 543561,1984

Behie, G. A. and Vinsome, P. K. W. "Block Iterative Method for Fully Implicit Reservoir Simulation”. Soc. of Pet. Eng. J., pages 658--668, Oct. 1982

Cao, H., Ph.D. Dissertation "Development of Techniques for General Purpose Simulators". Stanford University, June 2002

Cao, H. and Aziz, K. "Performance of IMPSAT and IMPSAT-AIM Models in Compositional Simulation". SPE 77720, SPE Annual Technical Conference and Exhibition held in San Antonio, Texas, 29 September-2 October 2002

Christie, M. A. and Blunt, M. J. "Tenth SPE Comparative Solution Project: A Comparison of Upscaling Techniques". SPE 66599, SPE Reservoir Simulation Symposium, TX, February 11-14, 2001

Coats, K.H.: “A Note on IMPES and Some IMPES Based Simulation Models”, SPEJ (SPE 65092), September, 2000

Demmel, W. J. “Applied Numerical Linear Algebra”. Society for Industrial and Applied Mathematics, Philadelphia, 1997 
Forsyth, P.A. and Sammon, P.H. "Practical Considerations for Adaptive Implicit Methods in Reservoir Simulation”, J. Comp. Phys., Vol. 62, pp265-281, 1986

Fu, D. X. “Numerical Simulation of Fluid Dynamics” (in Chinese). Beijing, 1993

Fussell, D. D. and Yanosik, J. L. "An Iterative Sequence for Phase-Equilibria Calculations Incorporating the Redlich-Kwong Equation of State" SPEJ, pp173182, June 1978

GeoQuest, Schlumberger "Eclipse 200 Reference Manual - Multi-Segment Wells", 2000 Release, 2000

GeoQuest, Schlumberger "Eclipse Technical Description 2001A”, 2001

Godunov, C. K. "Difference Method for Computing the Discontinuity in Fluid Dynamics". Math Sbornik, 1959, 47(3):271

Harten A. "High Resolution Schemes for Hyperbolic Conservation Laws”. J. Comput Phys, 49:357 393, 1983

Jiang, Y. "Tracer Flow Modeling and Efficient Solvers for GPRS”, M.S. Report, Stanford University, Petroleum Engineering, 2004

Landmark Graphics Corporation: “BlitzPak User’s Guide”, 1998, http://www.lgc.com

Lake, W. L. "Enhanced Oil Recovery”. Prentice-Hall, Inc, University of Texas, Austin, 1989

Lien, F. S. and Leschziner website: http://mecheng1.uwaterloo.ca/ fslien/tvd/ umist. html. 1994

Liu, R. X. and Shu, Q. W. "Several New Methods in Computational Fluid Dynamics" " (in Chinese). Hefei, 2003

Palagi, C. Ph.D. dissertation "Generation and Application of Voronoi Grid to Model Flow in Heterogeneous Reservoirs". Chapter 10, "Dual Timesteps to Model Tracer Flow", Stanford University, 1992

Pan, H. Q. "Test Plan and Test Case Specifications for Testing General Purpose Research Simulator (GPRS) Software”. Personal communication, October, 2003 
Rubin, B. and Blunt, M. J. "Higher-Order Implicit Flux Limiting Schemes for Black Oil Simulation". $11^{\text {th }}$ SPE Symposium on Reservoir Simulation held in Anaheim, California, February 17-20, 1991

Ruge, J. W. and Stuben, K. "Multigrid Methods -Chapter 4 Algebraic Multigrid". SIAM, 1987

Russell, T. F. "Stability Analysis and Switching Criteria for Adaptive Implicit". SPE 18416 , SPE Symposium on Reservoir Simulation in Houston, TX, February 6-8, 1989

Saad, Y. and Schultz, M.: "GMRES: A Generalized Minimum Residual Algorithm for Solving Non-symmetric Linear Systems", SIAM J. Sci. Statist. Comput., Vol. 7, pp856-869, 1986

Stone, H. L. and Garder, A. O., Jr. "Analysis of Gas-Cap or Dissolved Gas Drive Reservoir". SPE J., June, 1961 (AIME Trans. Vol. 222)

Stuben, K. "Algebraic Multigrid (AMG): Experiences and Comparisons", proceedings of the International Multigrid Conference, Copper Mountain, CO, April 6-8, 1983

Stuben, K. and Clees, T. “User's Manual of SAMG”, Release 21c August, 2003

Thomas, G. W. and Thurnau, D. H. "Reservoir Simulation Using Adaptive Implicit Method". SPE J. , October, 1983

Wallis, J. R. "Incomplete Gaussian Elimination as a Preconditioning for Generalized Conjugate Gradient Acceleration". SPE 12265, this paper was presented at the Reservoir Simulation Symposium held in San Francisco, CA, November 15-18, 1983

Wallis, J. R., Kendall, R. P., Little, T. E. "Constraint Residual Acceleration of Conjugate Residual Methods". SPE 13536, this paper was presented at the 1985 Reservoir Simulation Symposium held in Dallas, Texas, February 10-13, 1985.

Wattenbarger, R.C., Aziz, K. and Orr, Jr. F. M. "High-Throughput TVD-Based Simulation of Tracer Flow". SPE 29097, Proceedings, 13th Symposium for 
Reservoir Simulation, San Antonio, Texas, February 12-15, 1995. Published SPE Journal, Vol 2, No. 3, pp. 254-267 (September 1997)

Zhang, H.X. and Shen, M.Y. "Computational Fluid Dynamics --- Fundamentals and Applications of Finite Difference Methods” (in Chinese). Beijing, 2003 


\section{Gridding and Upscaling for 3D Unstructured Systems}

This chapter reports some of the developments and results presented by Mathieu Prévost in his $\mathrm{PhD}$ thesis (Prévost, 2003). For some of the grid generation aspects of this work, we interacted closely with Francois Lepage and Jean-Laurent Mallet at Ecole Nationale Supérieure de Géologie in Nancy, France (Prof. Mallet is the original developer of the GoCad geological modeling software). The work performed at Nancy was not part of the DOE funded work. However, in order to maintain completeness in this chapter, we include some description of the Nancy gridding developments (in section 3.3 below).

\subsection{Basic Issues with Unstructured Models}

The recent evolution of reservoir simulation is toward more structurally complex geological models and increasingly detailed petrophysical property descriptions. In order to manage reservoir uncertainties, reservoir simulation studies may now also require multiple models to be generated, often with different geological scenarios. It is a key challenge to generate gridded reservoir descriptions that incorporate the structural complexity of the geology while maintaining model sizes that are practical for flow simulations. A general approach for addressing the high level of detail in geocellular models is to upscale; i.e., introduce a significant degree of grid coarsening. Flexible (unstructured) grids provide an attractive solution for this coarsening step, as they enable the accurate and efficient modeling of both the reservoir geometry and heterogeneity.

Several issues arise with unstructured grids, particularly within the context of grid generation and upscaling. The data structure must enable the construction of grids that conform to geometrically complex 3D surfaces and honor complex surface intersections and topology constraints. This issue is handled by a sophisticated gridding framework called a Soft Frame Model. In this work, grids are generated to conform not only to geological features but are also used to introduce higher resolution in regions of high flow. This is accomplished by solving a representative flow problem on the fine scale and using the flow information in the grid construction step. Upscaled properties must then be computed for these unstructured flow-based grids. This work proposes a novel approach for this upscaling problem. Finally, a grid quality 
assessment based on streamline simulations for tracer flow is proposed. For this aspect of the problem, we generalize streamline simulation techniques to unstructured grids.

The outline of this chapter is as follows. We first present the basic issues and workflow for reservoir simulation with unstructured grids. Next we describe the techniques used for grid generation (both in terms of structure and flow resolution). Then, we discuss the generation of the coarse model, specifically the upscaling procedure, and the development of a streamlinebased simulator capable of obtaining flow responses on (unstructured) coarse models. Finally, we illustrate the overall methodology on several example cases.

\subsection{Overview of the Gridding and Upscaling Procedure}

Our starting point is a fine scale geocellular model, which may be defined on an unstructured or a structured grid, as well as a description of the key structural geological features. Our overall procedure is then as follows. For a particular set (or sets) of boundary or well conditions, we perform a fine scale steady-state single-phase flow simulation; i.e., we solve

$$
\nabla \cdot(\mathbf{k} \nabla p)=q
$$

where $\mathbf{k}$ is the permeability tensor, $p$ is pressure and $q$ represents source terms. This calculation is generally not overly expensive because it need only be performed once. From this solution, we compute the Darcy velocity $\mathbf{u}$ via $\mathbf{u}=-\mathbf{k} \nabla p$ and then generate streamlines. The behavior of an incompressible tracer can then be modeled by tracking particles along streamlines. This is a very inexpensive calculation for structured models. Next, using the flow information as well as structural information, we generate a coarse scale unstructured tetrahedral grid. This represents the primal grid. The dual grid is constructed by forming control volumes that are centered at the nodes (vertices) of the primal grid.

We upscale the underlying fine scale geocellular description to the scale of the control volumes. This provides the coarse scale model. We discretize the governing equations on the dual (coarse) grid using a control volume finite element (CVFE) method (Verma and Aziz, 1997). In this method, fluxes across control volume faces are represented in terms of the cellcentered pressures (as in flux continuous discretizations on structured or unstructured grids; e.g., Edwards, 2001; Aavatsmark et al., 1998; Lee et al., 2002). These fluxes may be defined in terms 
of the pressures for only the two cells sharing the interface (in this case we have a two-point flux approximation or TPFA) or in terms of these cell pressures as well as those of other neighboring cells (multipoint flux approximation or MPFA). Next, we perform the same steady-state singlephase flow simulation on the coarse grid as was run on the fine scale. This allows us to compare the flow responses of the two models and to iterate on the coarse grid structure if necessary. The tracer solution on the coarse scale is accomplished using a streamline simulator developed especially for CVFE solutions on unstructured grids.

The overall work flow described above for the construction of unstructured simulation models is illustrated schematically in Fig. 3-1. There are several new elements in this methodology, including the unstructured grid generation, the procedures for transmissibility upscaling and streamline simulation on unstructured grids, and the linkage of the fine scale flow simulation to the grid generation procedure. We now consider each of these issues in turn.

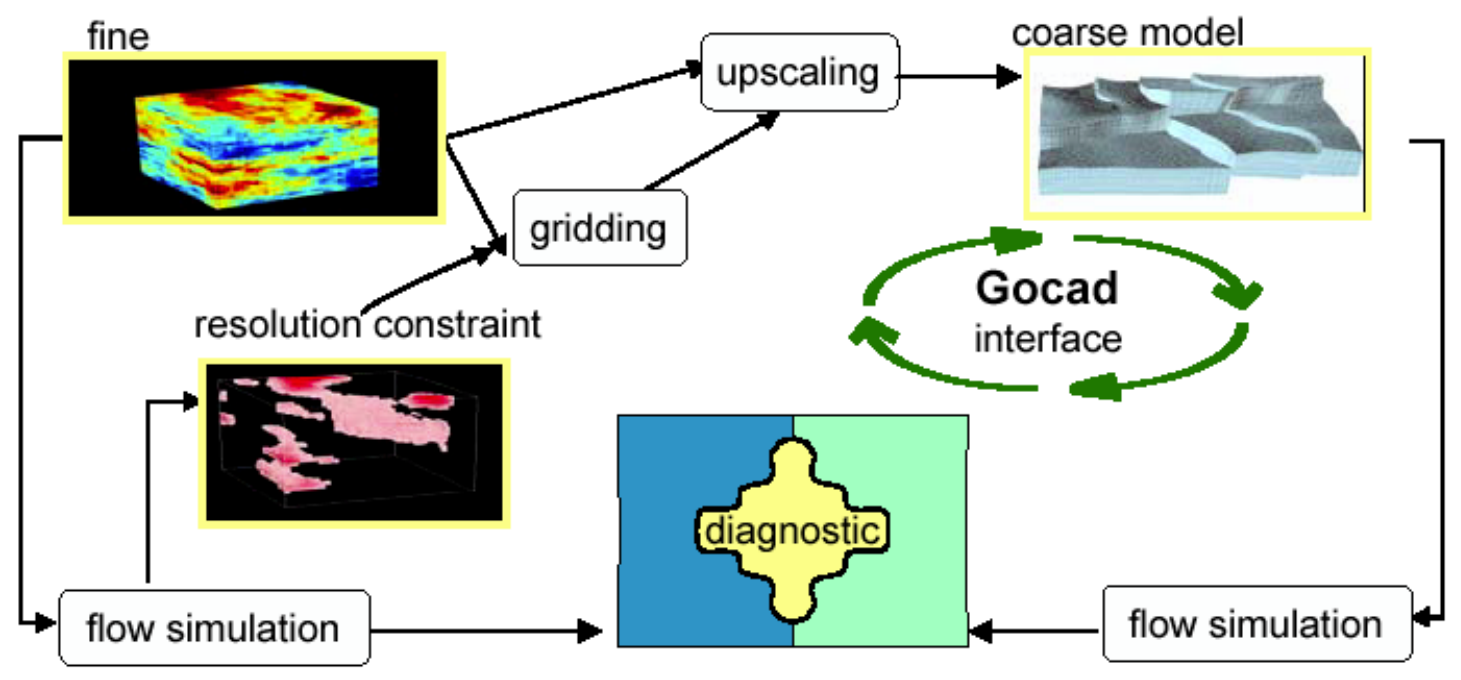

Figure 3-1: Schematic of overall workflow for generation of unstructured simulation model

\subsection{Grid Generation Methodology}

The proposed methodology relies on a flow-based, quality controlled grid construction. It starts from a structural model that must first be "enriched" to ensure that it provides a valid representation of the topological constraints. Then, appropriate gridding algorithms operate on 
the initial grid and adapt the cell shape and size to user-defined resolution constraints while maintaining the topological and geometrical validity of the grid. This construction relies on a topological structure that embeds the concepts of a grid structural model as well as grid topology and resolution constraints.

This section briefly describes the key aspects of the gridding framework. First, the data structures developed for the grid generation methodology are considered, followed by a discussion of the grid generation algorithms. Finally, we describe how the flow response from the fine scale property model is incorporated into the grid generation. Note that the grid generation may be applied as the first step of the overall procedure (i.e., to define the fine scale model) or it may not be used until the coarse grid generation step.

\subsubsection{Soft Frame Model}

From a geometrical point of view, a structural model is a set of discrete 3D representations of geological objects such as faults, horizons, and their borders. Objects of dimension $p(p$ in $[0,2])$ are represented by grids comprised of elements of dimension $p$ called "p-cellular complexes". These objects and their borders have various topologies (e.g., they can be either closed or open). Building such structural models is a crucial research topic and numerous techniques have been proposed (see e.g., Caumon et al., 2004). In the following, we will consider the structural model to be an input.

In general, every p-cellular complex is built independently; i.e., surfaces representing faults and horizons do not share any borders (even though they may physically intersect). In order to construct a volumetric mesh that accounts for the potentially very complex contacts between the geological objects, we require a "valid" topological and geometrical representation of the contacts. This is achieved using a macro model called a Soft Frame Model (Lepage, 2003). With this representation, a contact of dimension $p$ (called a p-radial element) is defined as the association of $p+1$ intersecting objects plus their $p$ intersections. Based on that definition, relative incidency and adjacency relationships are defined. These allow us to represent any structural model with an incidency graph. The ensemble of all radial elements forms a Soft Frame Model. This representation enables us to manipulate the contacts efficiently and flexibly. Furthermore, the geometrical consistency (every contact has a unique geometry shared by all the intersecting objects that define it) is ensured by generating meshes for all the radial elements of the Soft 
Frame Model, incorporating known sets of geometrical constraints such as nodes, edges or faces. This approach is conceptually very different from the standard proposed solutions based on the computation of the geometrical intersections between objects (see e.g., Caumon et al., 2004).

\subsubsection{Grid Refinement}

Within our gridding framework, meshes are constructed as a series of constrained cellular complexes, from lower to higher dimensions (points, lines, surfaces and volumes), where lower dimensional objects serve as constraints for higher dimensional objects. To honor constraints in the triangulation, we apply a conforming technique, which tends to produce high-quality triangles in terms of shape. This is the case because the resulting triangles are all strongly Delaunay. The process of honoring constraints in the triangulation may require the insertion of extra points (Steiner points) in the mesh, in contrast to boundary-constrained Delaunay meshes, where the circumcircle of some of the triangles may contain other points. A key problem with these insertions is the possible lack of convergence of the gridding algorithm when the constraining elements bear small angles. Our technique successfully addresses this issue by applying appropriate bisection rules expressed in the low-distorted parameterized spaces where triangulations are built.

Ensuring a satisfying shape for a tetrahedron is more difficult than for a triangle. Indeed, it is impossible to build a well-shaped tetrahedron based on a quasi-degenerated face. As described before, a conforming approach may be used to honor constraints in the tetrahedralization. However, it would produce far too many Steiner points in practice (Shewchuck, 2002), which is in contradiction to our objective. A standard boundary-constrained Delaunay methodology was therefore applied. It uses finite sequences of edge and face swaps plus a point insertion mechanism to recover constraints in the mesh.

To optimize the shape of the resulting tetrahedra, we propose an approach with no insertion of Steiner points. This produces tetrahedra with a bounded circumradius-to-shortest-edge ratio in a portion of the mesh, while keeping the boundary-constrained Delaunay property. Badly shaped tetrahedra are removed from the mesh by inserting their circumcenter into the tetrahedralization, as in any Delaunay refinement technique. 


\subsubsection{Flow Adaptation}

Using the Soft Frame Model and gridding algorithms presented above, we generate flow-adapted grids according to the following sequence. We perform incompressible tracer flow simulations on the reference geocellular model for one or more sets of boundary conditions. While the flow responses thus obtained serve as a reference for the diagnostic that controls the iterations of our overall methodology (Fig. 3-1), the overall solution is used to generate a velocity map for each flow problem. Then, with appropriate scalings, this information is averaged into a mean flow rate map that is then transformed into a target resolution constraint. The appropriate use of the flow information is the real challenge of the grid adaptation. This issue is addressed here by defining gridding parameters and investigating the search space in order to find optimum combinations that minimize the number of simulation cells while preserving the flow response. These gridding parameters include the aspect ratio (or anisotropy) of the tetrahedral grid elements. The layered aspect of petrophysical property distributions suggests the use of elongated tetrahedra. This can be achieved by computing element sizes using a metric (rather than plain Euclidean distance) when searching for elements that violate the resolution constraint. The size ratio between small elements (used in high flow regions) and large elements is another important grid parameter. The sensitivity of simulation results to several of the key gridding parameters is reported in Prévost (2003).

\subsection{Transmissibility Upscaling}

Upscaled properties must be calculated for the coarse grid. The dual cells of the flow-adapted grid (obtained as described above) are the control volumes used in the CVFE discretization. The objective of the upscaling step is to provide the flow simulator with (dual) cell to cell transmissibility coefficients that relate flow across an interface to values of the pressure at the center of neighboring (dual) cells. These coefficients must capture the flow effects of the underlying fine scale permeability description. The transmissibilies in the upscaled model can be calculated in at least two different ways. The first method, referred to as a $\mathbf{k}^{*}$-MPFA upscaling, entails the calculation of an upscaled permeability tensor for each control volume and the subsequent determination of the multipoint flux CVFE stencil from the $\mathbf{k}^{*}$ values in neighboring 
cells (this approach is akin to the method of Wen et al., 2003, for structured grids). The second method, referred to as a $T^{*}$-TPFA upscaling, entails a direct computation of the two-point transmissibility coefficients between pairs of connected cells. This approach is analogous to techniques used within structured finite difference simulation; see, e.g., Chen et al. (2003). Both the $\mathbf{k}^{*}$ and $T^{*}$ upscaling procedures were implemented and evaluated (Prévost, 2003). Numerical tests showed that the $T^{*}$-TPFA method is generally more robust and accurate for the types of problems tested. We now describe the $T^{*}$ calculation in more detail.

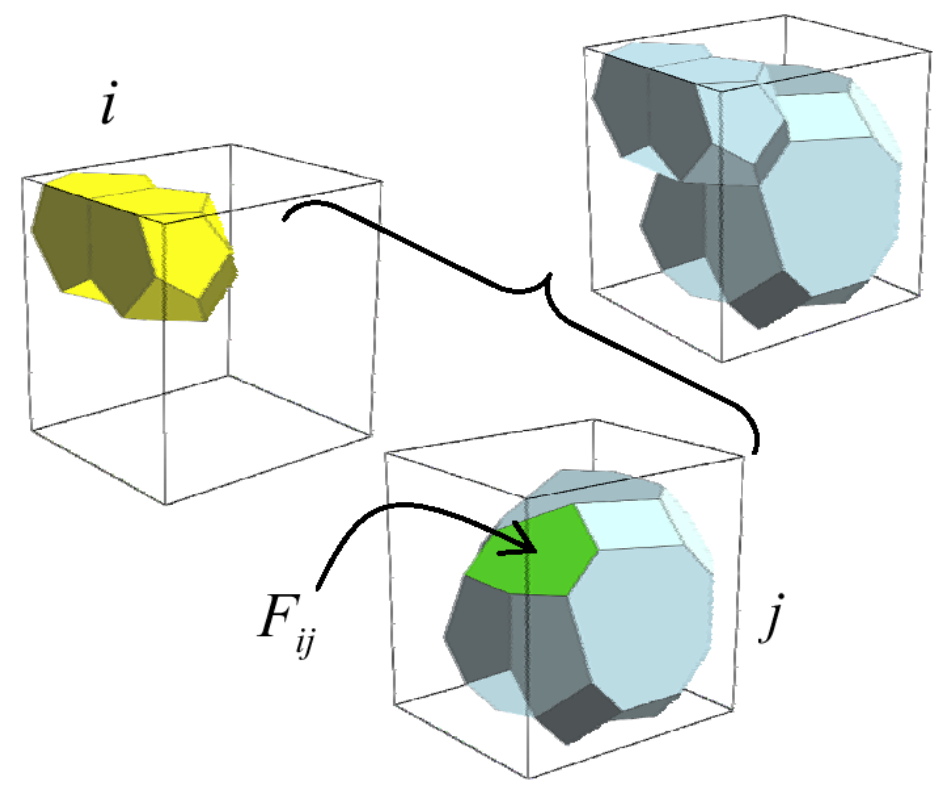

Figure 3-2: Upscaled permeability $\mathbf{k}^{*}$ is calculated on cells $i$ and $j$, while upscaled transmissibility $T^{*}$ is computed directly over the interface $F_{i j}$

Consider an interior face of the dual grid and the two corresponding adjacent cells (as shown in Fig. 3-2). The two cells are polyhedra and the face is a polygonal surface which, in the case of a CVFE dual grid, is in general nonplanar. In TPFA, the flux across a control volume face is written in terms of the difference between pressure values at the centers of the dual cells. Denoting $F_{i j}$ as the face between cells $i$ and $j$, the flux $q_{i j}$ across $F_{i j}$ can be written as:

$$
q_{i j}=-T_{i j}\left(p_{j}-p_{i}\right)
$$


where $T_{i j}$ is the two-point transmissibility coefficient.

We compute $T_{i j}$ by assembling and solving a local flow problem, as illustrated Fig. 3-3. The local domain includes the two control volumes that share the target interface as well as a small border region around the two cells. We solve the single-phase pressure equation subject to pressure - no flow boundary conditions over this fine scale region and then compute average pressures over cells $i$ and $j$, designated $\left\langle p_{i}\right\rangle$ and $\left\langle p_{j}\right\rangle$. The average Darcy velocity can also be calculated over fine scale cells contained in the interface $F_{i j}$, allowing the determination of the total flux $q_{i j}^{c}$ across $F_{i j}$. The upscaled transmissibility is then calculated via:

$$
T_{i j}^{*}=-\frac{q_{i j}^{c}}{\left\langle p_{i}\right\rangle-\left\langle p_{j}\right\rangle}
$$

We note that this transmissibility upscaling procedure requires that a local problem be solved for each connection (interface) in the domain, which can be somewhat time consuming.

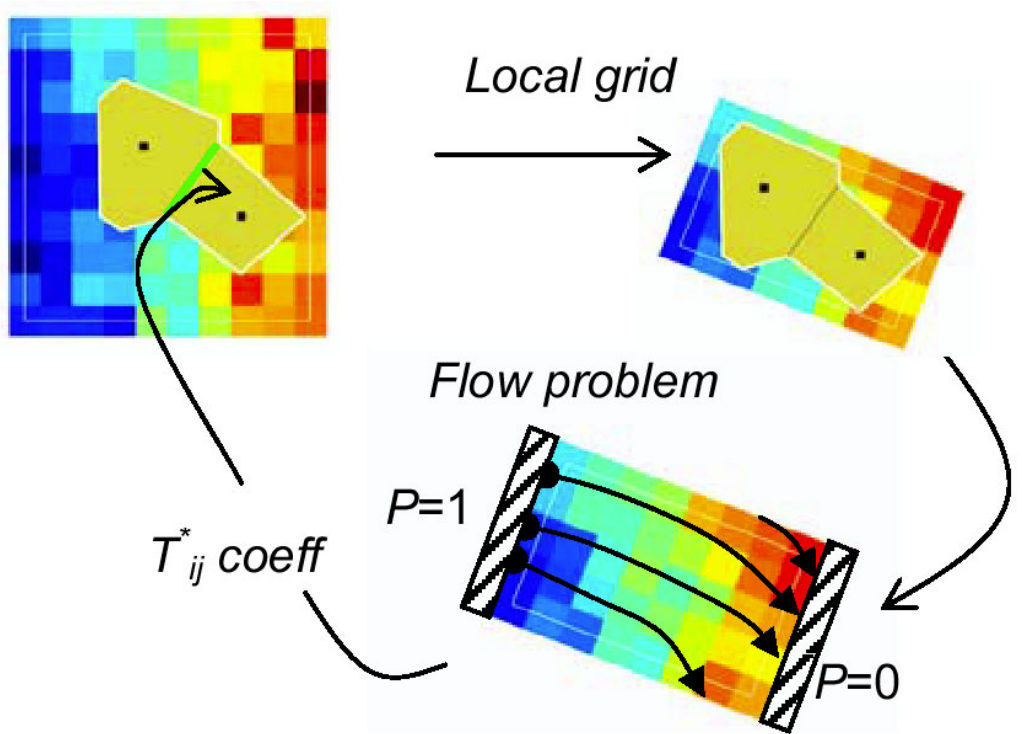

Figure 3-3: Upscaled transmissibility $T^{*}$ is computed by solving a local single-phase problem on the rotated grid (illustrated here in 2D) 


\subsection{Streamline Simulation on Unstructured Grids}

As indicated earlier, we use a simplified flow model (incompressible tracer flow) to gauge the accuracy of the coarsened reservoir description. This model will of course not correspond exactly to the actual physical situation of interest in most cases, but it represents a reasonable approximation for purposes of coarse model evaluation. We apply streamline simulation for this calculation, as it is one of the most efficient techniques for simulating incompressible tracer flow. If the boundary or well conditions do not change during the course of the simulation, the complete production history can be obtained with only one solution of the pressure equation. The producing fraction of the injected tracer is computed by tracking a number of streamlines from injectors to producers and plotting, for each producer, the streamline time of arrival (sorted in increasing order) versus the cumulative sum of the rates associated with the streamlines. For simple one-producer one-injector cases, the time axis of the production curves can easily be expressed in terms of pore volume injected.

The quality of the coarse model is assessed based on the quantitative level of agreement between the flow-response obtained on the fine and coarse grids for one or more flow problems. In most cases, we use tracer flow simulations from one face of the reservoir to the other (under fixed pressure boundary conditions) and compare total flow rates across the model and nondimensional fractional flow curves (i.e., water cut). For that purpose, a streamline-based simulator for 3D unstructured grids was developed. We note that the streamline simulator allows for the use of any set of boundary or well conditions.

The principal difficulty in the extension of the streamline method to unstructured grids lies in the determination of an appropriate analytical velocity interpolant, which is required for the tracing. Our technique consists of a local postprocessing of the numerically calculated fluxes, leading to a consistent and flux-continuous piecewise constant representation for the velocity. Fluxes are obtained from the pressure field computed using the CVFE discretization scheme.

For each control volume, the postprocessing introduces a subgrid comprised of tetrahedra, each of which is associated with an unknown velocity vector. The velocity vectors are then

constrained to satisfy certain physically meaningful constraints: flux-continuity, consistency with 
the fluxes derived from the CVFE solution, and minimization of the rotational component of velocity. The local constraints are expressed as a linear system of equations which scales as the number of tetrahedra shared by the control volume. Solution of this set of equations (for each control volume) provides a velocity field that can be used for streamline tracing. See Prévost (2003) for full details.

As an illustration of the performance of the unstructured streamline simulation method, we show a result for which comparison to a structured grid streamline simulation (Batycky et al., 1997) is possible. We consider a homogeneous cubic domain and simulate flow from one corner to the diagonally opposite corner. For the unstructured simulations, a total of 1728 control volumes were used (the unstructured grid used is shown Fig. 3-4); for the structured simulations a total of 27,000 cells were used (though similar results could be obtained with fewer cells). Results for water cut $\left(F_{w}\right)$ versus PVI are shown in Fig. 3-5. The excellent agreement between the two calculations demonstrates the accuracy of our unstructured streamline simulations.

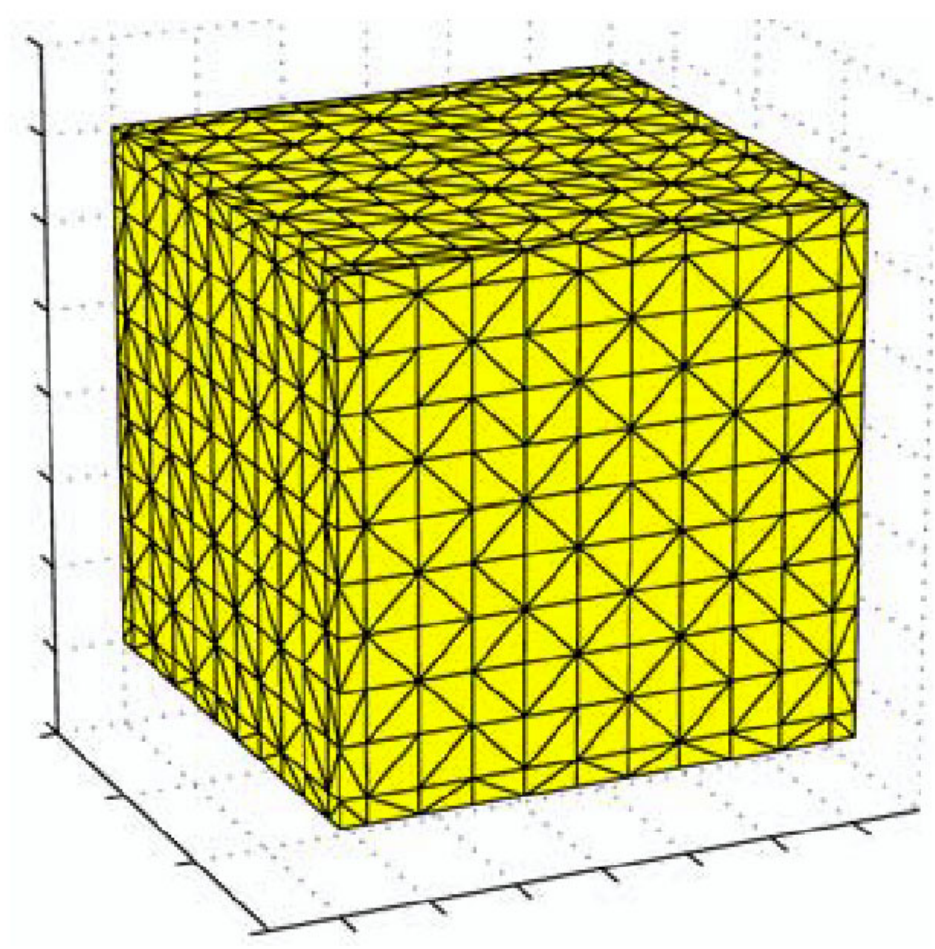

Figure 3-4: Unstructured grid used for streamline simulation (1728 cells) 


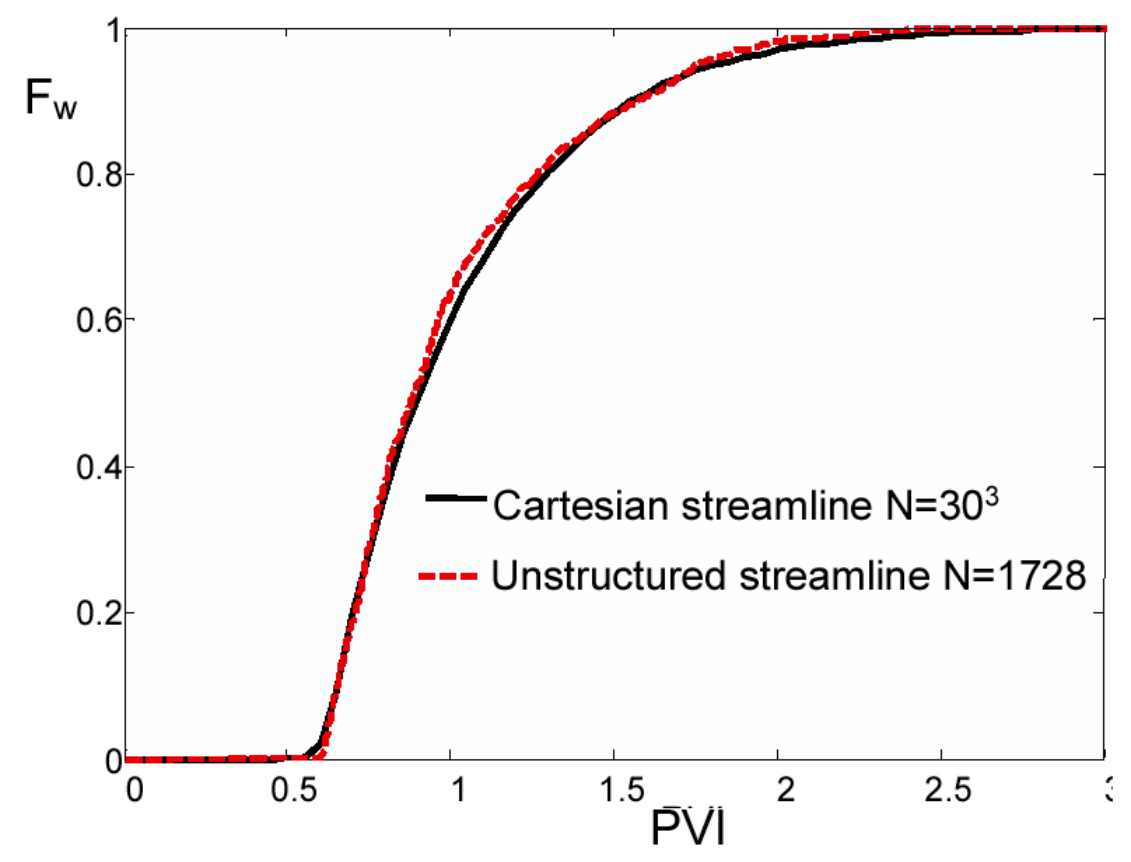

Figure 3-5: Comparison of unstructured streamline simulation results to those from a Cartesian grid streamline simulation

\subsection{Results for Unstructured Grid Generation and Upscaling}

We now present examples demonstrating the capabilities of the general grid generation procedure as well as flow results illustrating the flow-based gridding and upscaling techniques. In all cases, a Soft Frame Model was built from an initial structural model, surfaces were remeshed using a conforming approach, volumes were tetrahedralized, optimized according to a shape criterion, and post-processed through an edge-swap technique.

Fig. 3-6 gives an illustration of the meshes produced by the grid generation methodology. The structural model is comprised of a "Y" fault with surfaces intersecting at low angles. The gridded model exhibits an accurate resolution of surface intersections and a very acceptable shape for the tetrahedra. Fig. 3-7 shows an example of the combination of structural resolution and flow adaptation. The fault geometry is honored in both the primal and the dual grid. The resolution constraint is provided by a mean flow rate map calculated on the fine model and 
averaged for different flow problems. The dual grid displays the norm of the upscaled permeability tensor. The unstructured coarse model can now be used in flow simulations.

Finally, we demonstrate the benefit of flow-adaptation using the methodology presented. We consider a fine $200 \times 100 \times 50$ Cartesian model with a layered, log-normally distributed permeability field of variance (in $\log k$ ) of 1 and dimensionless correlation lengths $l_{x}=1.0, l_{y}=0.75$ and $l_{z}=0.2$ (shown in Fig. 3-8). The flow adaptation was performed in three steps: (1) determine the optimum target aspect ratio from uniformly coarsened grids, (2) determine the optimum large-to-small cell size ratio, (3) select the grid size (number of cells) to achieve the desired level of accuracy (based on the flow diagnostics).

The optimum aspect ratio is defined as the aspect ratio that provides the most accurate coarse models (relative to the reference solution). For this case it was found to be $10 \times 5 \times 1$ (i.e., a well shaped tetrahedron would fit in an ellipsoid with principal axis lengths in the ratios 10:5:1). Then, enforcing this optimal aspect ratio for the tetrahedral cells, grid adaptation was performed. This required the determination of the optimum large-to-small tetrahedra size ratio and the selection of the desired number of coarse cells. The performance of the resulting "optimum" coarse model (relative to the fine model) is shown in Fig. 3-9. Here, $Q_{c}$ is the ratio of the total flow rate through the coarse model to that of the fine model $\left(Q_{c}=1\right.$ indicates exact agreement). Using 1394 control volumes, the flow-rate adapted grid provides an error in total flow of less than $1 \%$ and very accurate results for water cut $\left(F_{w}\right)$ relative to the fine $\left(10^{6}\right.$ cells $)$ model. The figure also shows results using a uniformly coarsened model with nearly twice as many cells as the flow-rate adapted grid. The errors in both flow rate and water cut are considerably higher with this model, demonstrating the benefits of the flow-rate adaptivity applied here. 


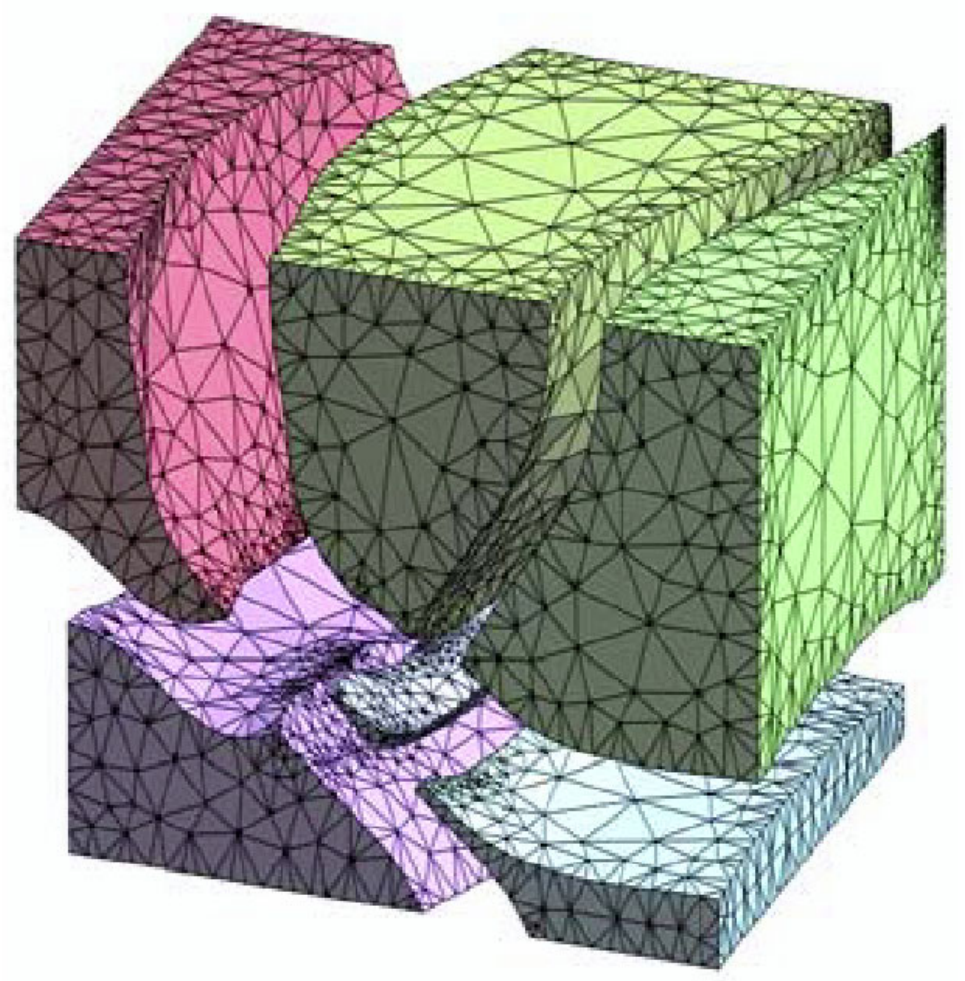

Figure 3-6: Unstructured tetrahedral grid of a complex structural model containing a "Y" fault (from Lepage, 2003)

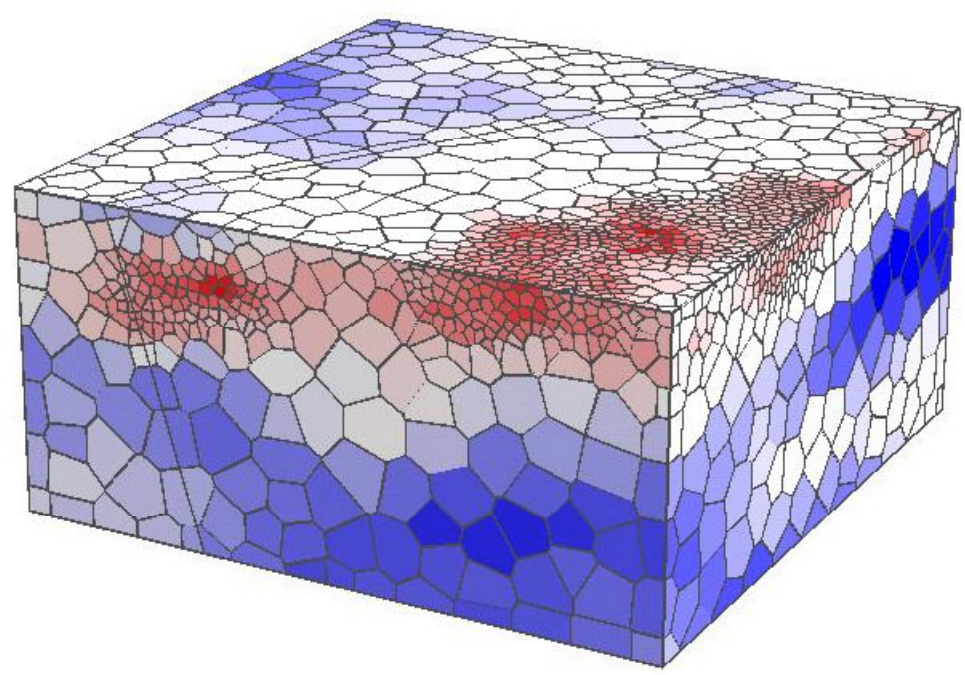

Figure 3-7: Dual grid honoring both flow and structural (fault) information 


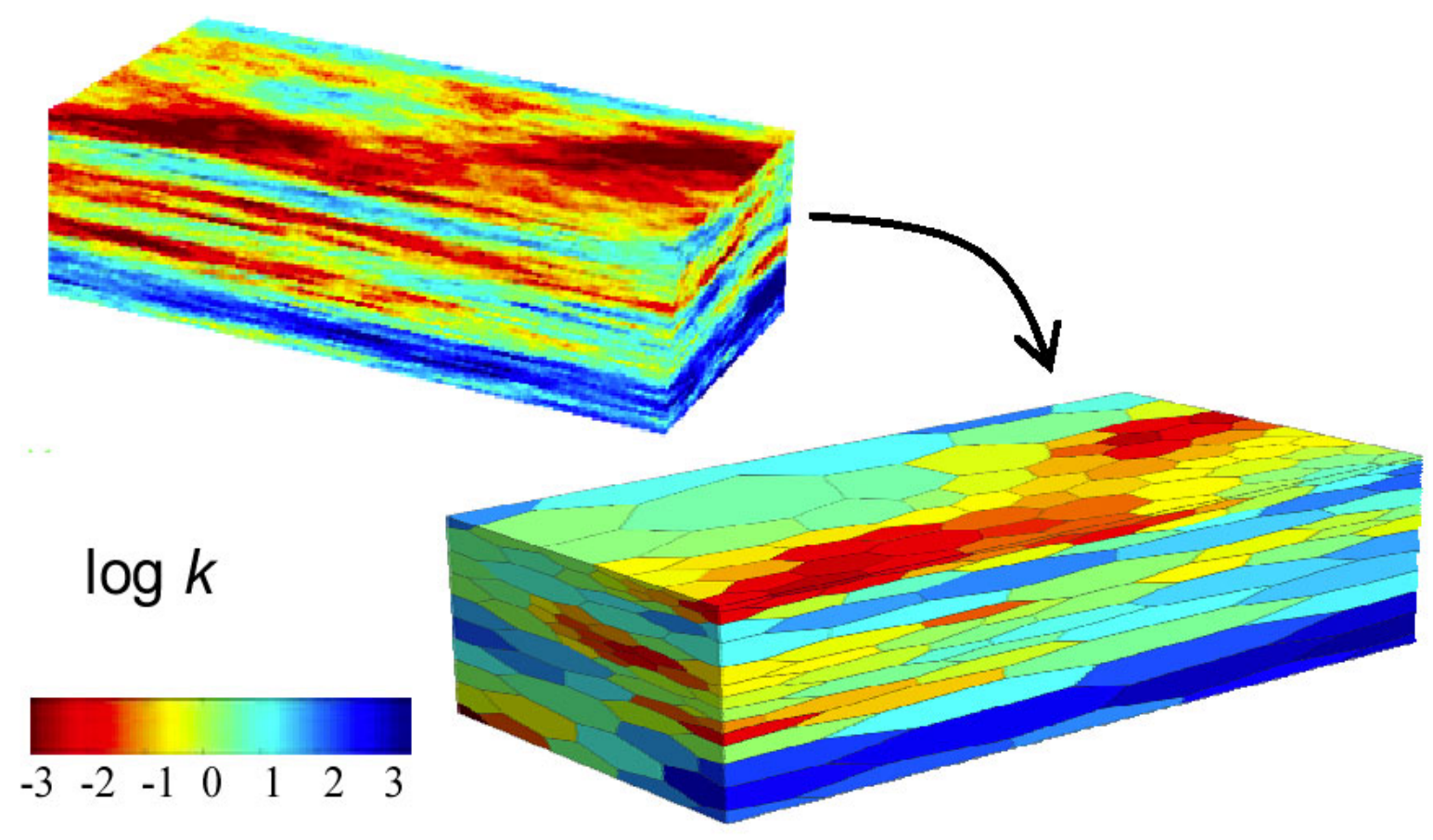

Figure 3-8: Fine scale permeability field (upper) and flow-rate adapted coarse scale dual grid

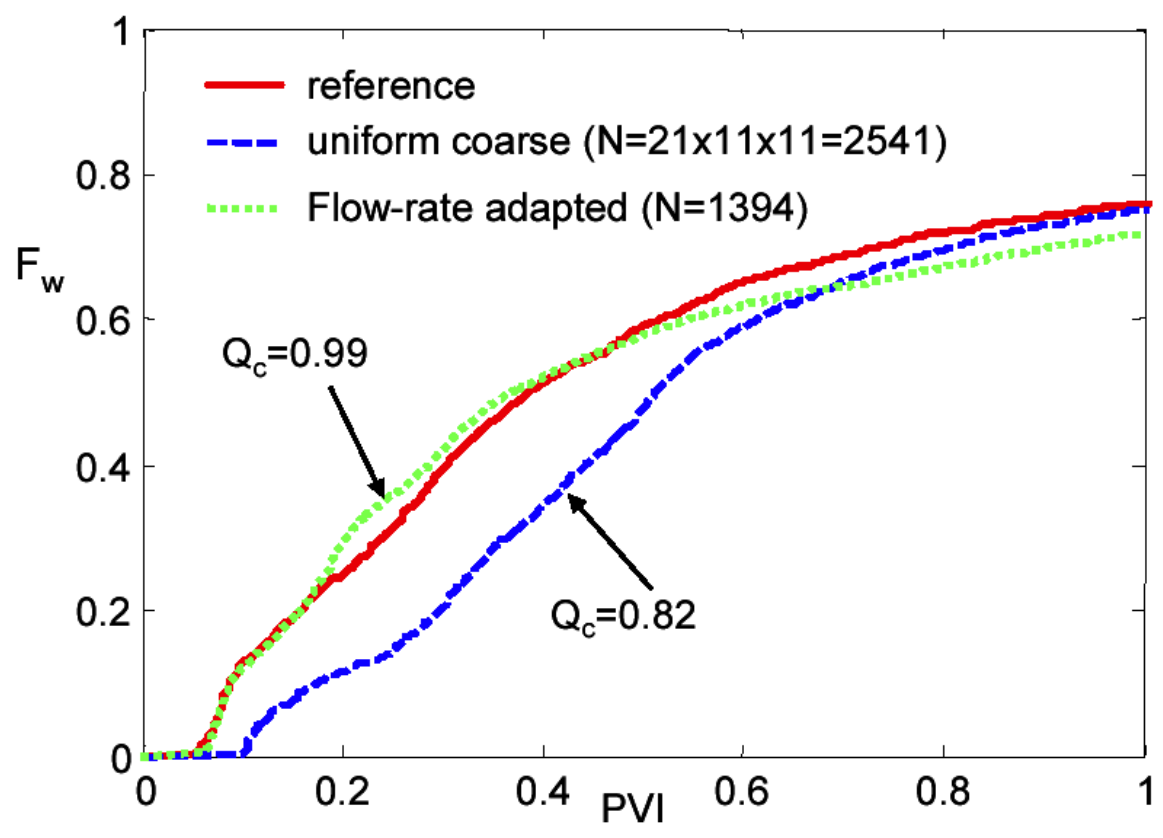

Figure 3-9: Fractional flow curves for tracer simulation for flow from left to right. Both total flow rate $\left(Q_{c}\right)$ and water cut for the coarse model are considerably improved by adaptation to flow rate information 


\subsection{Summary}

The work presented here can be summarized as follows:

- A methodology for gridding and upscaling to unstructured 3D systems was developed and applied. The gridding procedure was based on recent developments from the GoCad modeling group.

- A method for streamline simulation on unstructured grids was developed and tested. The method applies a post-processing of CVFE simulation results and was shown to provide results of a high degree of accuracy.

- The use of flow-adapted gridding used in conjunction with transmissibility upscaling was shown to provide accurate results for a heterogeneous example case.

- The streamline simulation and upscaling techniques presented here are computationally demanding. Future work should be directed toward accelerating these procedures. It will also be of interest to further compare $\mathbf{k}^{*}$ (multipoint flux) upscaling with $T^{*}$ (two-point flux) upscaling.

\subsection{References}

Aavatsmark, I., Barkve, T., and Mannseth, T.: "Control-Volume Discretization Methods for 3D Quadrilateral Grids in Inhomogeneous, Anisotropic Reservoirs, SPEJ, 3, 146-154, 1998.

Batycky, R.P., Blunt, M.J., and Thiele, M.R.: "A 3D Field-Scale Streamline-Based Reservoir Simulator," SPERE, 12, 246-254, 1997.

Caumon, G., Lepage, F., Sword, C. H., and Mallet, J.-L.: "Building and Editing a Sealed Geological Model,” Math. Geol., 36, 405-424, 2004.

Chen, Y., Durlofsky, L.J., Gerritsen, M., and Wen, X.H.: "A Coupled Local-Global Upscaling Approach for Simulating Flow in Highly Heterogeneous Formations," Adv. Water Resources, 26, 1041-1060, 2003.

Edwards, M.G.: "Split Full Tensor Discretization Operators for Structured and Unstructured Grids in Three Dimensions," paper SPE 66358 presented at the SPE Reservoir Simulation Symposium, Houston, Feb. 11-14, 2001.

Lee, S.H., Tchelepi, H.A., Jenny, P., De Chant, L.: "Implementation of a Flux-Continuous Finite-Difference Method for Stratigraphic, Hexahedron Grids," SPEJ, 7, 267-277, 2002.

Lepage, F.: "Three-Dimensional Mesh Generation for the Simulation of Physical Phenomena in Geosciences," PhD thesis, Institut National Polytechnique de Lorraine (France), 2003. 
Prévost, M.: "Accurate Coarse Reservoir Modeling Using Unstructured Grids, Flow-based Upscaling and Streamline Simulation," PhD thesis, Stanford University, 2003.

Shewchuk, J.S.: "Constrained Delaunay Tetrahedralization and Provably Good Boundary Recovery," Proceedings of the $11^{\text {th }}$ International Meshing Roundtable, Sandia National Laboratories, 193-204, 2002.

Verma, S.K. and Aziz, K.: "A Control Volume Scheme for Flexible Grids in Reservoir Simulation," paper SPE 37999 presented at the SPE Reservoir Simulation Symposium, Dallas, June 8-11, 1997.

Wen, X.H., Durlofsky, L.J., and Edwards, M.G.: "Upscaling of Channel Systems in Two Dimensions Using Flow-Based Grids," Transport in Porous Media, 51, 343-366, 2003. 


\section{Part II. Coupling of the Reservoir and Nonconventional Wells in Simulators}

In this part of the report we first (Chapter 4) describe the development of semianalytical solution techniques for modeling nonconventional wells producing under single-phase flow, as well as the determination of well index for finite difference simulation. This work has been covered extensively in previous DOE reports and includes contributions from a number of MS and PhD students (Christian Wolfsteiner, Per Valvatne, Jerome Serve and Hiroshi Fukagawa). These developments have been incorporated into a code (AdWell) that has been distributed to and used by a number of companies. This code is maintained and enhanced by Dr. Huanquan Pan, a research associate within our group. The material discussed below has been published in several papers (e.g., Wolfsteiner et al., 2000, 2003; Valvatne et al., 2001, 2003) and has also been presented previously in MS and $\mathrm{PhD}$ theses as well as in previous Annual Reports to DOE. The presentation below covers much of our developments, but for a fuller description the reader is referred to the relevant theses, previous DOE reports and publications.

In Chapter 5 we describe a drift-flux model for multiphase wellbore flow and the determination of appropriate model parameters applicable for flow in large diameter pipes and wells. This research involved a significant experimental program (led by Dr. Gary Oddie at Schlumberger Cambridge Research; the experimental work is outside the scope of this DOE funded work) and the subsequent determination of model parameters for realistic systems. This modeling work has (or soon will) appear in two SPE papers (Shi et al., 2003, 2004). Contributors to this work include MS students Banu Alkaya and Luis Rodrigo Díaz Terán Ortegón, post-doctoral researcher Dr. Hua Shi, and Schlumberger GeoQuest researcher Dr. Jonathan Holmes. Dr. Holmes developed and implemented the original drift-flux model on which this work is based. 


\section{Numerical Calculation of Productivity Index and Well Index for Nonconventional Wells}

In reservoir simulation models, the wellbore is not modeled explicitly but is rather linked to the reservoir through use of a well model. Well models, such as those due to Peaceman (1983), represent the well production (or injection) from grid block $i, j, k$ in terms of a well index $(W I)$, the wellbore pressure $\left(p_{w b}\right)$ and the pressure of the grid block $\left(p_{i, j, k}\right)$ via:

$$
q_{i, j, k}=W I\left(p_{w b}-p_{i, j, k}\right),
$$

where $q_{i, j, k}$ represents the flow rate from the well into block $(i, j, k)$. The form of Eq. 4-1 is very similar to the general expression for well productivity index $(P I)$ :

$$
q=P I\left(p_{w b}-<p>\right),
$$

where $q$ is now total well production and $\langle p\rangle$ is the average reservoir pressure. The similarity between Eqs. 4-1 and 4-2 illustrates why techniques developed for the calculation of PI can also be applied to the calculation of WI. In this chapter we describe the development and application of efficient semianalytical approaches for the calculation of $P I$ and WI for nonconventional wells.

An efficient approach for modeling the productivity of nonconventional wells operating under primary production is to employ a semianalytical solution technique. Early work along these lines included single horizontal wells (of infinite conductivity) aligned parallel to one side of a box shaped reservoir. Solution methods were successive integral transforms (Goode and Thambynayagam, 1987; Kuchuk et al., 1988) and the use of instantaneous Green's functions (Daviau et al., 1985; Clonts and Ramey, 1986; Ozkan et al., 1989; Babu and Odeh, 1989), resulting in infinite series expressions. More complex well geometries were considered later (Economides et al., 1996; Maizeret, 1996; Ouyang and Aziz, 2001) with the application of numerical integration. A number of works (see Ouyang, 1998, and citations

therein) include coupling of wellbore hydraulics (i.e., finite conductivity wells) with reservoir flow.

All of the semianalytical techniques mentioned above have the advantage of limited data requirements and high degrees of computational efficiency relative to finite difference 
simulation. This makes them well suited for use as screening tools or for approximate simulations of primary production. Previous semianalytical techniques are, however, limited to homogeneous systems or at most strictly layered systems (Lee and Milliken, 1993; Basquet et al., 1998). This represents a substantial limitation because the productivity of nonconventional wells can be significantly impacted by fine scale heterogeneities in the nearwell region. Fine scale heterogeneity can be incorporated into detailed simulation models, though the resulting models may be complex to build and may require substantial computation time to run.

In this work we extend existing semianalytical approaches in many important directions. We introduce a new model to approximately account for heterogeneity in the near-well region. This is accomplished by introducing an effective skin $s$ into the semianalytical model and then estimating this effective skin as a function of position along the wellbore. This concept is sufficiently general to enable the modeling of real reservoirs, as will be demonstrated below. We also generalize existing semianalytical techniques to enable the modeling of downhole inflow control devices (chokes). These devices allow for the independent control of each branch or segment of a multilateral well and can be used to produce from multiple reservoirs (at different pressures) with a single well. Other developments include the extension of the method to handle fixed pressure wells (rather than just fixed rate wells) and to model gas reservoirs. In the case of gas reservoirs the semianalytical approach may be slightly less accurate, as the linearization of the governing equation introduces some error. We also develop a procedure for the computation of the well index for finite difference simulation. This requires the post-processing of the semianalytical solution and a subsequent finite difference calculation with well block rates specified.

This chapter proceeds as follows. We first describe the basic semianalytical solution method and then indicate briefly how the various extensions indicated above are implemented. We then present numerical results for horizontal and multilateral wells in heterogeneous three dimensional systems. These results are in many cases compared with detailed finite difference calculations to assess their level of accuracy. Next we describe our approach for the calculation of well index. A numerical example demonstrates the applicability of this procedure to a deviated well in an unstructured grid. 


\subsection{Semianalytical Formulation}

The semianalytical approach and the permeability model based on effective skin are described in this section. Further details are available in previous work (e.g., Wolfsteiner et al., 2000; Valvatne et al., 2003, and our previous DOE Annual Reports).

\subsubsection{Problem Formulation}

Within the semianalytical context, the reservoir is modeled as a parallelepiped with any combination of constant potential or no-flow boundary conditions on the six bounding faces. Isothermal flow in the reservoir can be described by the single-phase pressure equation for slightly compressible fluids:

$$
\nabla \cdot(\mathbf{k} \nabla \Phi)=\phi \mu c_{t} \frac{\partial \Phi}{\partial t}
$$

with appropriate initial and boundary conditions. Eq. 4-3 is formulated using potential $\Phi=p+\left(g / g_{c}\right) \rho z$ rather than pressure $p$ to account for gravity effects. The permeability $\mathbf{k}$ is assumed to be a diagonal tensor, therefore, Eq. 4-3 can be written as

$$
k_{x x} \frac{\partial^{2} \Phi}{\partial x^{2}}+k_{y y} \frac{\partial^{2} \Phi}{\partial y^{2}}+k_{z z} \frac{\partial^{2} \Phi}{\partial z^{2}}=\phi \mu c_{t} \frac{\partial \Phi}{\partial t}
$$

Wells can have an arbitrary configuration and trajectory (Fig. 4-1). Each well $i_{w}$ (of a total of $n_{w}$ wells) can have $n_{l}\left(i_{w}\right)$ laterals which in turn consist of $n_{s}\left(i_{w}, i_{l}\right)$ segments. The total number of segments $N_{s}$ is then given by

$$
N_{s}=\sum_{i_{w}=1}^{n_{w}} \sum_{i_{w}=1}^{n_{l}\left(i_{w}\right)} n_{s}\left(i_{w}, i_{l}\right)
$$




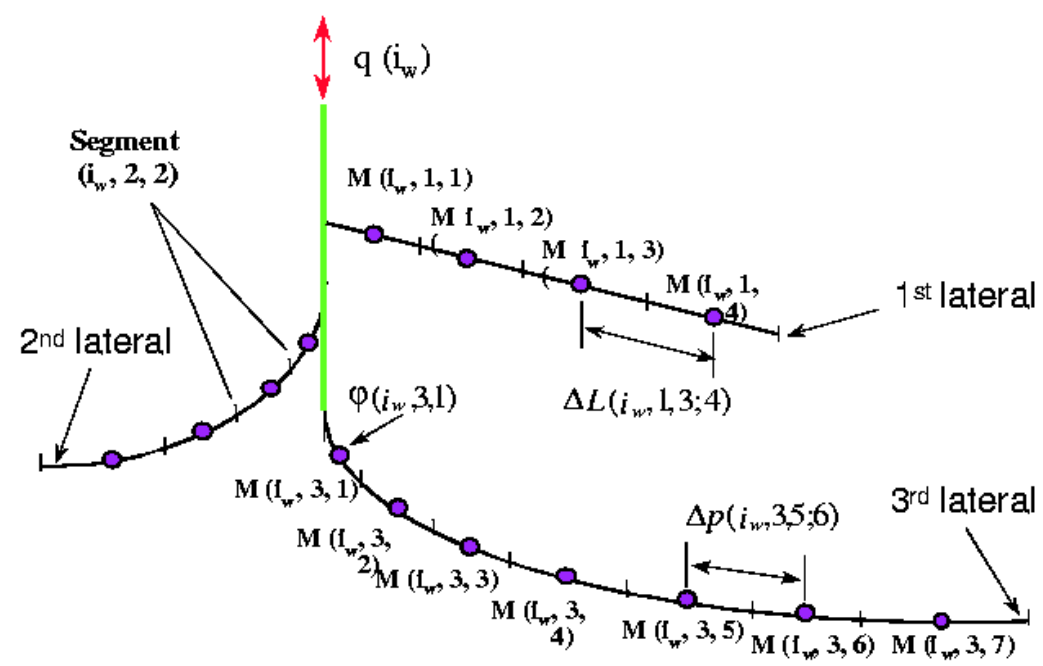

Figure 4-1: Arbitrary well configuration (from Ouyang and Aziz, 2001)

\subsubsection{Transformation of Anisotropic Reservoir System}

To simplify the solution of the potential equation (Eq. 4-4), the reservoir dimensions are transformed such that the anisotropic reservoir can be modeled as an isotropic equivalent (Besson, 1990). The transformation is given by:

$$
\left[\begin{array}{l}
x^{\prime} \\
y^{\prime} \\
z^{\prime}
\end{array}\right]=\left[\begin{array}{lll}
a & 0 & 0 \\
0 & b & 0 \\
0 & 0 & c
\end{array}\right]\left[\begin{array}{l}
x \\
y \\
z
\end{array}\right]
$$

where $(x, y, z)$ are the coordinates in the original (anisotropic) system and $\left(x^{\prime}, y^{\prime}, z^{\prime}\right)$ are the coordinates in the isotropic equivalent system. The parameters $a, b$, and $c$ are given by:

$$
a=\frac{\sqrt{k_{y y} k_{z z}}}{k}, b=\frac{\sqrt{k_{x x} k_{z z}}}{k}, c=\frac{\sqrt{k_{x x} k_{y y}}}{k}, k=\sqrt[3]{k_{x x} k_{y y} k_{z z}}
$$

In transformed coordinates, Eq. 4-4 can be expressed as

$$
\frac{\partial^{2} \Phi}{\partial x^{\prime 2}}+\frac{\partial^{2} \Phi}{\partial y^{\prime 2}}+\frac{\partial^{2} \Phi}{\partial z^{\prime 2}}=\frac{\phi \mu c_{t}}{k} \frac{\partial \Phi}{\partial t}
$$

The wellbore radius of a well having an arbitrary trajectory is now (Besson, 1990): 


$$
r_{w}^{\prime}=\frac{r_{w}}{\alpha^{1 / 3}} \frac{1}{2 \beta} \sqrt{\left(1+\frac{\beta^{2}}{\gamma}\right)^{2}+\left[\left(\delta-\frac{1}{\delta}\right) \frac{\cos \theta \cos \varphi \sin \varphi}{\gamma}\right]^{2}}
$$

where

$$
\begin{aligned}
& \alpha=c^{3 / 2}=\sqrt{\frac{\sqrt{k_{x} k_{y}}}{k_{z}}} \\
& \beta=\sqrt{\frac{a}{b} \cos ^{2} \varphi+\frac{b}{a} \sin ^{2} \varphi} \\
& \gamma=\sqrt{\cos ^{2} \theta+\left(\frac{a^{2}}{c^{2}} \cos ^{2} \varphi+\frac{b^{2}}{c^{2}} \sin ^{2} \varphi\right) \sin ^{2} \theta} \\
& \delta=\sqrt{\frac{k_{x}}{k_{y}}}
\end{aligned}
$$

The angles $\theta$ and $\varphi$ are the inclination and azimuth of the well, respectively. $\theta=90^{\circ}$ represents a horizontal well, and $\varphi=0^{\circ}$ indicates a well that is parallel to the $x$ axis. The spatial transformation of Eq. 4-6 results in a deformation of the circular well section into an ellipse. The equivalent wellbore radius of the transformed well is given by the arithmetic average of the major and minor axes of this elliptic section. The elliptic section can be obtained by using two anisotropy ratios $\alpha$ and $\delta$, and two geometrical factors $\beta$ and $\gamma$. See Besson (1990) for further details.

\subsubsection{Dimensionless Variables}

Dimensionless time $t_{D}$, inflow rate $q_{D}$ and potential $\Phi_{D}$ can be defined as follows: 


$$
\begin{aligned}
t_{D} & =\frac{k t}{C_{t} \phi \mu c_{t} x_{e}^{\prime 2}} \\
q_{D} & =\frac{q B \mu}{C_{q} k x_{e}^{\prime 2} \Phi_{i}} \\
\Phi_{D} & =\frac{\Phi_{i}-\Phi}{\Phi_{i}} \\
x_{D} & =\frac{x^{\prime}}{x_{e}^{\prime}}, y_{D}=\frac{y^{\prime}}{x_{e}^{\prime}} \text { and } z_{D}=\frac{z^{\prime}}{x_{e}^{\prime}}
\end{aligned}
$$

The length of the transformed reservoir in the $x$-direction is designated as $x_{e}^{\prime}$.

\subsubsection{Wellbore/Reservoir Coupling Model}

The coupled reservoir-wellbore model has $2 \times N_{s}$ unknowns. These are the dimensionless inflow (or outflow) $q_{D}\left(i_{w}, i_{l}, i_{s}\right)$ and potential drawdown $\Phi_{w D}\left(i_{w}, i_{l}, i_{s}\right)$ at the midpoint $M\left(i_{w}, i_{l}, i_{s}\right)$ of each segment for well $i_{w}$ (Fig. 4-1). We therefore need the same number of equations to describe the system. These equations are obtained from mass balances, potential drawdown and wellbore hydraulics relationships.

\subsubsection{Reservoir Flow}

The mass balance requires that the production or injection rate in each well should be equal to the sum of the contributions from each segment in the well:

$$
\sum_{i_{l}=1}^{n_{l}\left(i_{w}\right)} \sum_{i_{s}=1}^{n_{s}\left(i_{w}, i_{l}\right)} q_{D}\left(i_{w}, i_{l}, i_{s}\right)=q_{D}\left(i_{w}\right)
$$

where $i_{w}=1,2, \ldots n_{w}$. According to the principle of superposition, the potential drawdown at any point in space can be expressed in terms of contributions from all sources/sinks in the system. If we consider the potential drawdown at the midpoints of each segment in the system, the principle of superposition yields

$$
\begin{aligned}
& \Phi_{w D}\left(j_{w}, j_{l}, j_{s}\right) \\
& =\sum_{j_{w}=1}^{n_{w}} \sum_{j_{l}=1}^{n_{l}\left(j_{w}\right)} \sum_{j_{s}=1}^{n_{s}\left(j_{w}, j_{l}\right)} q_{D}\left(j_{w}, j_{l}, j_{s}\right) \Phi_{D}\left(j_{w}, j_{l}, j_{s}\right)\left[M\left(i_{w}, i_{l}, i_{s}\right)\right]
\end{aligned}
$$


where $\Phi_{D}\left(j_{w}, j_{l}, j_{s}\right)\left[M\left(i_{w}, i_{l}, i_{s}\right)\right]$ is the dimensionless potential drawdown at the midpoint of segment $j_{s}$ of lateral $j_{l}$ of well $j_{w}$ caused by the source/sink with unit strength of segment $i_{s}$ of lateral $i_{l}$ of well $i_{w}$.

The dimensionless potential drawdown can be calculated from the instantaneous point sink/source functions. As an example, for a one-dimensional system with no-flow boundaries at dimensionless locations 0 and 1, the dimensionless potential drawdown at point $x$ at time $t$ caused by the instantaneous source with unit strength at $x_{0}$ and initial time is given by:

$$
\begin{aligned}
S_{x}\left(x, x_{0}, t_{D}\right) & =2 \sum_{k=1}^{\infty} \exp \left(-k^{2} \pi^{2} t_{D}\right) \sin k \pi x \cos k \pi x \\
& =\frac{1}{\sqrt{4 \pi} t_{D}} \sum_{k=-\infty}^{\infty}\left[\exp \left(\frac{\left(x-2 k-x_{0}\right)^{2}}{-4 t_{D}}\right)+\exp \left(\frac{\left(x-2 k+x_{0}\right)^{2}}{-4 t_{D}}\right)\right]
\end{aligned}
$$

These formulas can be shown to be equivalent. Both expressions involve infinite series, however, the exponential form converges faster for small $t$, while the trigonometric expression has an advantage for large $t$. According to Neuman's product rule, the threedimensional problem is solved by multiplying the one-dimensional solutions of Eq. 4-14. An integration over time of the three-dimensional solution yields a continuous solution:

$$
\begin{array}{r}
\Phi_{D}\left(j_{w}, j_{l}, j_{s}\right)\left[M\left(i_{w}, i_{l}, i_{s}\right)\right]=\frac{1}{x_{D e} y_{D e} z_{D e}} \int_{0}^{t_{D}} S_{x}\left(\frac{x_{D}}{x_{D e}}, \frac{x_{D 0}}{x_{D e}}, \frac{t_{D}}{x_{D e}}\right) \\
S_{y}\left(\frac{y_{D}}{y_{D e}}, \frac{y_{D 0}}{y_{D e}}, \frac{t_{D}}{y_{D e}}\right) S_{z}\left(\frac{z_{D}}{z_{D e}}, \frac{z_{D 0}}{z_{D e}}, \frac{t_{D}}{z_{D e}}\right) d t_{D}
\end{array}
$$

Finally, an integration of the point sources/sinks over the well segment $\left[M_{0}, M_{1}\right]$ yields the drawdown expression for each segment midpoint:

$$
\begin{aligned}
& \Phi_{D}\left(j_{w}, j_{l}, j_{s}\right)\left[M\left(i_{w}, i_{l}, i_{s}\right)\right] \\
& =\int_{M_{D 0}}^{M_{D 1}}\left[\Phi_{D}\left(j_{w}, j_{l}, j_{s}\right)\left[M\left(j_{w}, j_{l}, j_{s}\right)\right]-\frac{1}{4 \pi r_{w D}}\right] d M_{D}+\int_{M_{D 0}}^{M_{D 1}} \frac{1}{4 \pi r_{w D}} d M_{D}
\end{aligned}
$$

The term $1 / 4 \pi r_{w D}$ is subtracted from the point source solution to prevent convergence problems in the integration. See Maizeret (1996) for the details related to this issue. 


\subsubsection{Wellbore Hydraulics}

Wellbore pressures of segments in a well are related to each other through wellbore hydraulics. The basic equation describing pressure losses in the wellbore can be expressed as

$$
\Phi_{w}\left(i_{w}, i_{l}, i_{s}+1\right)=\Phi_{w}\left(j_{w}, j_{l}, j_{s}\right)+\Delta p_{f}\left(i_{w}, i_{l}, i_{s} ; i_{s}+1\right)+\Delta p_{a}\left(i_{w}, i_{l}, i_{s} ; i_{s}+1\right)
$$

Here $\Delta p_{f}\left(i_{w}, i_{l}, i_{s} ; i_{s}+1\right)$ represents frictional pressure loss between $M\left(j_{w}, j_{l}, j_{s}\right)$ and $M\left(i_{w}, i_{l}, i_{s}+1\right)$ and $\Delta p_{a}\left(i_{w}, i_{l}, i_{s} ; i_{s}+1\right)$ represents pressure loss caused by acceleration effects between $M\left(j_{w}, j_{l}, j_{s}\right)$ and $M\left(i_{w}, i_{l}, i_{s}+1\right)$. These values are flow-rate dependent. Expressions for $\Delta p_{f}$ and $\Delta p_{a}$ are given below, along with equations for pressure losses in smart wells equipped with downhole chokes.

\subsubsection{Solution of the System}

Since the starting segments at the heel of each lateral are close together, we can assume $\Phi_{w D}\left(i_{w}, 1,1\right) \approx \Phi_{w D}\left(i_{w}, i_{l}, 1\right)$. Eq. $4-17$ can then be written as

$$
\begin{aligned}
& \Phi_{w D}\left(i_{w}, i_{l}, i_{s}\right) \\
& =\Phi_{w D}\left(i_{w}, 1,1\right)-\frac{1}{\Phi_{i}} \sum_{i=1}^{i_{s-1}}\left[\Delta p_{f}\left(i_{w}, i_{l}, i_{s} ; i_{s}+1\right)+\Delta p_{a}\left(i_{w}, i_{l}, i_{s} ; i_{s}+1\right)\right]
\end{aligned}
$$

The number of potential drawdown unknowns is reduced to $n_{w}$. By substituting Eq. 4-18 into Eq. 4-13, we have

$$
\begin{aligned}
& \Phi_{w D}\left(i_{w}, 1,1\right)-\frac{1}{\varphi_{i}} \sum_{i=1}^{i_{s}-1}\left[\Delta p_{f}\left(i_{w}, i_{l}, i_{s} ; i_{s}+1\right)+\Delta p_{a}\left(i_{w}, i_{l}, i_{s} ; i_{s}+1\right)\right] \\
& =\sum_{j_{w}=1}^{n_{w}} \sum_{j_{l}=1}^{n_{l}\left(j_{w}\right)} \sum_{j_{s}=1}^{n_{s}\left(j_{w}, j_{l}\right)} q_{D}\left(j_{w}, j_{l}, j_{s}\right) \Phi_{D}\left(j_{w}, j_{l}, j_{s}\right)\left[M\left(i_{w}, i_{l}, i_{s}\right)\right]
\end{aligned}
$$

The wellbore hydraulics equation introduces nonlinearity into the solution of Eq. 4-19, so some type of iterative procedure is required to solve this problem. Ouyang and Aziz (2001) introduced a successive substitution approach for cases where the nonlinearity is relatively weak (e.g., cases without downhole chokes). Valvatne et al. (2000, 2003) applied Newton's method for situations where the successive substitution may encounter difficulty due to the 
tight coupling caused by downhole chokes. The Newton procedure is the approach used in the examples presented here.

\subsubsection{Incorporation of Skin into Solution}

Skin factors can be introduced into the coupled wellbore/reservoir model described in the previous section. Based on the assumption that the skin effect around a segment $M\left(i_{w}, i_{l}, i_{s}\right)$ only contributes to the wellbore potential drawdown of that segment, Eq. 4-13 can modified to include the skin effect $(s)$ :

$$
\begin{aligned}
\Phi_{w D}\left(i_{w}, i_{l}, i_{s}\right)= & \sum_{j_{w}=1}^{n_{w}} \sum_{j_{l}=1}^{n_{l}\left(j_{w}\right)} \sum_{j_{s}=1}^{n_{s}\left(j_{w}, j_{l}\right)} q_{D}\left(j_{w}, j_{l}, j_{s}\right) \Phi_{D}\left(j_{w}, j_{l}, j_{s}\right)\left[M\left(i_{w}, i_{l}, i_{s}\right)\right] \\
& +q_{D}\left(i_{w}, i_{l}, i_{s}\right) s\left(i_{w}, i_{l}, i_{s}\right)
\end{aligned}
$$

This approach can introduce numerical problems when skin is highly negative. Valvatne (2000) found that a more stable approach is to use an effective wellbore radius $r_{w e}$ rather than the actual radius $r_{w}$, where $r_{w e}=r_{w} e^{-s}$. For the most difficult cases, a rescaling of skin is necessary to ensure the numerical stability. This rescaling reduces the magnitude of the maximum negative skin to a value that makes the equivalent wellbore radius less than half of the segment length. The calculation of the skin factor in highly heterogeneous systems is described next.

\subsection{Practical Computation of $s$ and $\mathbf{k}^{*}$}

In our semianalytical procedure, the permeability heterogeneity is represented in terms of just a few parameters. These include the background or overall "average" permeability, designated $\mathbf{k}^{*}$, and the near-well skin $s$. This permeability representation is therefore referred to as the $s$ - $\mathbf{k}^{*}$ model. The background permeability is constant throughout the reservoir, while the skin varies along the wellbore (i.e., a particular value of skin is associated with each well segment). The way in which $s$ and $\mathbf{k}^{*}$ are computed can have a significant impact on the accuracy of the model. Practical procedures for these and related calculations are described in this section. 


\subsubsection{Approaches for the Computation of Global Permeability}

Global permeability (designated $\mathbf{k}^{*}$ ) represents an effective overall permeability for the system. Within our semianalytical context, $\mathbf{k}^{*}$ is assumed to be a diagonal tensor (with components $k_{x x}^{*}, k_{y y}^{*}$ and $k_{z z}^{*}$ ). One approach to compute $\mathbf{k}^{*}$ is to solve the single-phase incompressible pressure equation numerically over the fine-scale geostatistical model. Such an approach is referred to as a flow-based technique. This calculation is performed with a prescribed pressure drop in each of the three coordinate directions to determine a diagonal tensor $\mathbf{k}^{*}$. This flow-based technique has been successfully applied for many cases (see e.g., results in Wolfsteiner et al., 2000 and Valvatne et al., 2003). This approach is not, however, appropriate for some geological settings. For example, when a reservoir contains shale layers, $k_{z z}^{*}$ calculated by the flow-based technique can be very small. Use of this $k_{z z}^{*}$ can result in a discrepancy in the pressure match between a finite difference method and the semianalytical approach. Reservoirs of this type may require a different approach for the calculation of $\mathbf{k}^{*}$. To address this issue, we describe an approach based on power averaging.

\subsubsection{Power Averaging Approach}

Journel et al. (1986) and Deutsch (1989) showed power averaging to be a viable approach to estimate effective permeability. Although the power-averaging technique entails only a few parameters, this approach is quite flexible and can be used to represent many types of geological settings by tuning the power-averaging coefficients. Furthermore, once we obtain a reasonable match for pressure between reference finite difference results and semianalytical results, it is expected that the tuned power averaging coefficients will be valid for other realizations with the same permeability statistics. The power-averaging approximation is given by:

$$
k_{d d}^{*}=\left(\frac{1}{n} \sum_{i=1}^{n} k_{d d}^{\omega_{d d}}\left(x_{i}\right)\right)^{1 / \omega_{d d}}
$$


The power coefficient $\omega(-1 \leq \omega \leq 1)$ can vary in each direction $d(d=x, y$ and $z)$. To obtain a close pressure match in the general case the tuning of $\omega$ is necessary. This is accomplished through comparison of the semianalytical solution with a few reference finite difference simulations (see Fukagawa, 2002, for details).

\subsubsection{Computation of Skin}

Durlofsky (2000) introduced a skin (designated $s$ ) as a means to account for near wellbore heterogeneity. This skin can be understood with reference to a radial flow problem. Fig. 4-2 depicts a simple radial system with two zones of permeability ( $k$ and $k_{a}$ ). The scalar background permeability is designated as $k$. The altered region around the wellbore, $r_{w}<r<$ $r_{a}$, has a different scalar permeability, $k_{a}$.

Hawkins (1956) defined the dimensionless skin for this system as

$$
s=\left(\frac{k}{k_{a}}-1\right) \ln \frac{r_{a}}{r_{w}}
$$

In our approach, $k$ is computed as the geometric mean of the diagonal components of $\mathbf{k}^{*}$; i.e., $k=\sqrt[3]{k_{x x}^{*} k_{y y}^{*} k_{z z}^{*}}$.

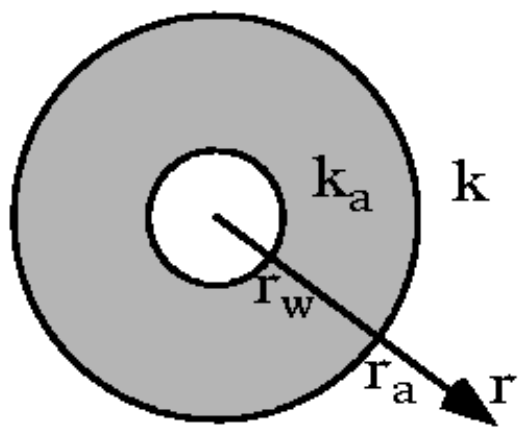

Figure 4-2: Flow in series in a radial system (from Wolfsteiner et al., 2000) 
The size of the altered permeability region, needed to determine $r_{a}$, can be determined from the structure of the permeability field. In simple systems, the correlation length was found to be an appropriate selection (Wolfsteiner et al., 2000). A rectangular region defined by the correlation lengths can also be taken as the "search region" that defines the altered zone (note that we use the terms "search region" and "altered zone" interchangeably).

\subsubsection{Computation of Permeability and Equivalent Radius in the Altered Zone}

The altered permeability $k_{a}$ is computed as a weighted power average of the permeabilities in the search region. This average is given by (Wolfsteiner et al., 2000):

$$
k_{a, d d}^{\xi}=\frac{1}{\Gamma_{a}} \int_{a} \frac{k_{d d}^{\xi}(\mathbf{x})}{r^{n}} d \mathbf{x}
$$

where $\xi$ is the averaging power, $r$ is the distance from the segment, $n$ is a distance weight, and the integration is evaluated over the search region $a$. The normalization $\Gamma_{a}$ is computed via $\Gamma_{a}=\int_{a} r^{-n} d \mathbf{x}$. The subscript $d d$ again indicates the directions of the diagonal tensor.

In order to account for the effects of local permeability anisotropy, we compute the following coefficients using the fine-scale permeabilities within the search region (Eq. 4-23):

$$
a_{L}=\frac{\sqrt{k_{a, y y} k_{a, z z}}}{k_{a}}, b_{L}=\frac{\sqrt{k_{a, x x} k_{a, z z}}}{k_{a}}, c_{L}=\frac{\sqrt{k_{a, x x} k_{a, y y}}}{k_{a}}, k_{a}=\sqrt[3]{k_{a, x x} k_{a, y y} k_{a, z z}}
$$

These coefficients represent the local anisotropy. Using these local coefficients, we perform the coordinate transform from the original system to a local coordinate system. We compute skin and perform other related calculations in this local system. However, the local coefficients and $k_{a}$ are dependent on each other through the distance $r$ (in Eq. 4-23), so we need an iterative procedure to compute $a_{L}, b_{L}, c_{L}$ and $k_{a}$ when anisotropy varies with position in the search region. This iterative procedure is described in Fukagawa (2002).

In the existing implementation, Eq. 4-23 is evaluated using a Monte Carlo type of integration (Wolfsteiner et al., 2000), which randomly samples the fine-scale permeabilities 
within the search region, excluding the wellbore itself. Throughout this study, we set $\xi=0$ (geometric average) and $n=2$ in Eq. $4-23$ as in previous studies.

Finally, we need to determine $r_{a}$ in Eq. 4-23 to compute a skin value. In a previous study (Wolfsteiner et al., 2000), $r_{a}$ was taken to be the geometric mean of the main axes forming the search region. Since the geometric mean (product of axes) could cancel out the effect of the local anisotropy ratio (Brigham, 1990), we now employ the radius of a circle in the transformed plane ( $u-v$ plane) as proposed by Peaceman (1983). Now $r_{a}$ can be expressed using transformed distance coordinates $u$ and $v$ as

$$
r_{a}=\sqrt{u^{2}+v^{2}}
$$

In cases where the well is not parallel to the $x-y-z$ coordinate system, the search region is rotated to be orthogonal to the segment. Then, $r_{a}$ can be determined using Eq. 4-25. The equivalent wellbore radius in Eq. 4-22 is calculated from Eq. 4-9 using the local anisotropy as computed using Eq. 4-24.

\subsection{Treatment of Downhole Inflow Control Devices}

We now briefly describe the models incorporated into our technique for the modeling of pressure losses due to wellbore flow and downhole chokes. The models used here are quite simple, though other models or correlations can be introduced easily into the general formulation. The well completion is modeled with separate segments representing tubing, annulus and downhole chokes.

For single-phase flow in the wellbore, there will be frictional $\left(\Delta p_{f}\right)$, acceleration $\left(\Delta p_{a}\right)$ and hydrostatic pressure losses. Hydrostatic effects are included automatically when losses are expressed in terms of potential rather than pressure. Significant acceleration effects occur in segments receiving large inflows. For a well segment of length $L$, diameter $D$, axial flow rate $q$ and inflow $q_{I}$, the losses are given by Ouyang et al. (1998):

$$
\begin{aligned}
& \Delta p_{f}=\frac{C_{f} f L \rho q^{2}}{D^{5}} \\
& \Delta p_{a}=\frac{C_{f} \rho q q_{I}}{D^{4}}
\end{aligned}
$$


where $f$ is the friction factor and the constant factor $C_{f}$ is $2.956 \times 10^{-12}$ in field units.

In this work we apply the formula suggested by Haaland (1981) to estimate $f_{0}$, the friction factor for turbulent flow in the absence of inflow or outflow. This friction factor requires modification to account for the effects of inflow or outflow from the well segment. The corrections suggested by Ouyang et al. (1998), which represent the wellbore friction (for well inflow) in the form:

$$
f=f_{0}\left(1-0.0153 R e_{w}^{0.3978}\right)
$$

where $R e_{w}$ is the wall Reynolds number (Reynolds number based on influx), were applied in this work.

Because downhole chokes are relatively new inventions, specific correlations for predicting pressure loss through these devices are not available in the literature. Additional complications arise because choking can be achieved using different methods depending on the system used. Sachdeva et al. (1986) proposed a two-phase choke model that is applicable for both critical and sub-critical flow (critical flow occurs when the flow rate through the choke is not affected by the upstream pressure). Because single-phase liquid flow at downhole conditions will rarely be critical, a simplified version of the model, applicable to single-phase, sub-critical liquid flow, was implemented. This model represents pressure losses through the choke $\Delta p_{c}$ via (Schlumberger, 1999):

$$
\Delta p_{c}=\frac{C_{c} \rho q^{2}}{C_{d}^{2} D_{c}^{4}}
$$

where $D_{c}$ is the choke diameter and the discharge coefficient, $C_{d}$, is taken to be 0.85 . The unit conversion constant $C_{c}$ is equal to $1.532 \times 10^{-8}$ in field units.

\subsection{Modeling Wells with Fixed Bottomhole Pressure}

The semianalytical formulation as described up to this point is appropriate when the total well rate (rather than wellbore pressure) is fixed in time. This limitation results from the form of the Green's functions used in the formulation.

We now describe a more general numerical treatment that allows the well rate to vary. This will occur, for example, when the bottomhole pressure is fixed. This more general 
formulation entails the use of an additional superposition in time, introduced to account for the variation in time of the segment rates. The wellbore potential in segment $\left(i_{w}, i_{s}\right)$ for a given time step $t_{n}$ is then written as follows:

$$
\begin{gathered}
\Phi_{w D}\left(i_{w}, i_{s}, t_{n}\right)=\Phi_{w D}\left(i_{w}, i_{s}, q_{1}, t_{n}\right)+\Phi_{w D}\left(i_{w}, i_{s}, q_{2}-q_{1}, t_{n}-t_{1}\right)+\ldots \\
\Phi_{w D}\left(i_{w}, i_{s}, q_{n}-q_{n-1}, t_{n}-t_{n-1}\right)
\end{gathered}
$$

In the following, the well rates $\left(q_{1}, q_{2}, \ldots, q_{n-1}\right)$ are assumed known at all time steps prior to $t_{n}\left(t_{1}, t_{2}, \ldots, t_{n-1}\right)$. The solution procedure then consists of two steps:

1. Applying the same general methodology as described for wells at constant rate, we compute the first $(n-1)$ potentials $\Phi_{w D}\left(i_{w}, i_{s}, q_{k}-q_{k-1}, t_{n}-t_{k-1}\right), k \in[1, n-1]$ with $q_{k}-q_{k-1}$ set constant over the interval $t_{n}-t_{k-1}$.

2. Then, using Eq. 4-30, we obtain a residual equation involving $q_{n}$. At $t_{n}$, the first stage implies the use of the $(n-1)$ Green's potentials $\Phi_{D}$ over each interval $t_{n}-t_{k-1}$. Indeed, for a given $k$, the potential drawdown in segment $\left(i_{w}, i_{s}\right)$ is now given by:

$$
\Phi_{w D}\left(i_{w}, i_{s}, q_{k}-q_{k-1}, t_{n}-t_{k-1}\right)=\sum_{j_{w}=1}^{n_{w}} \sum_{j_{s}=1}^{n_{s}\left(j_{w}\right)}\left(q_{I D}^{k}-q_{I D}^{k-1}\right)_{\left(j_{w}, j_{s}\right)} \Phi_{D}\left(j_{w}, j_{s}, i_{w}, i_{s}, t_{n}-t_{k-1}\right)
$$

where $\left(q_{I D}^{k}-q_{I D}^{k-1}\right)_{\left(j_{w}, j_{s}\right)}$ is the difference of inflow rates for segment $\left(j_{w}, j_{s}\right)$ between time steps $t_{k-l}$ and $t_{k}$.

Green's potentials are linear in time, so for a given $k$ they can be decomposed as follows:

$$
\Phi_{D}\left(j_{w}, j_{s}, i_{w}, i_{s}, t_{k}\right)=\Phi_{D}\left(j_{w}, j_{s}, i_{w}, i_{s}, t_{k-1}, t_{k}\right)+\Phi_{D}\left(j_{w}, j_{s}, i_{w}, i_{s}, t_{k-1}\right)
$$

where $\Phi_{D}\left(j_{w}, j_{s}, i_{w}, i_{s}, t_{k}\right)$ is equivalent to $\Phi_{D}\left(j_{w}, j_{s}, i_{w}, i_{s} 0, t_{k}\right)$. Using this linearity and assuming the time stepping interval to be constant, i.e., $\forall k, t_{k}-t_{k-1}=C$, where $C$ is a constant, the Green's potential $\Phi_{D}$ over the interval $t_{n}-t_{k-1}$ can be written as:

$$
\Phi_{D}\left(j_{w}, j_{s}, i_{w}, i_{s}, t_{n}-t_{k-1}\right)=\Phi_{D}\left(j_{w}, j_{s}, i_{w}, i_{s}, t_{n-k+1}\right)
$$


where the Green's potential $\Phi_{D}\left(j_{w}, j_{s}, i_{w}, i_{s}, t_{n-k+1}\right)$ has already been evaluated at all time steps prior to $t_{n}$. Then, at $t_{n}$, we only need to evaluate $\Phi_{D}\left(j_{w}, j_{s}, i_{w}, i_{s}, t_{n-1}, t_{n}\right)$ which, using Eq. 4-32, gives us $\Phi_{D}\left(j_{w}, j_{s}, i_{w}, i_{s}, t_{n}\right)$.

Once the first $(n-1)$ potentials $\Phi_{w D}\left(i_{w}, i_{s}, q_{k}-q_{k-1}, t_{n}-t_{k-1}\right)$ are computed, the second step consists of combining Eq. 4-30 and Eq. 4-18 (wellbore flow equation). We then obtain a residual equation involving $q_{n}$ :

$$
\begin{aligned}
R_{p}\left(i_{w}, i_{s}\right)= & \sum_{j_{w}=1}^{n_{w}} \sum_{j_{s}=1}^{n_{i s}\left(j_{w}\right)}\left(\frac{B \mu\left(q_{I}\left(j_{w}, j_{s}, t_{n}\right)-q_{I}\left(j_{w}, j_{s}, t_{n-1}\right)\right)}{k^{*} x_{e} \Phi_{i}}\right) \Phi_{D}\left(j_{w}, j_{s}, i_{w}, i_{s}, t_{n}-t_{n-1}\right) \\
& +\sum_{k=2}^{n-1} \Phi_{w D}\left(i_{w}, i_{s}, q_{k}-q_{k-1}, t_{n}-t_{k-1}\right) \\
& -\frac{1}{\Phi_{i}}\left[\Phi_{i}-\Phi_{w}\left(i_{w}\right)-\sum_{k_{s}=1}^{k_{s}=i_{s}}\left(\Delta p_{f}\left(i_{w}, k_{s}\right)+\Delta p_{a}\left(i_{w}, k_{s}\right)\right)\right]
\end{aligned}
$$

We thus compute the well rate $q_{n}$ at time $t_{n}$ such that the target bottomhole pressure, $\Phi_{w}\left(i_{w}\right)$, is obtained. Notice that at this last stage, the size (order) of the Jacobian is equal to the total number of segments in the system. The Jacobian matrix is now formed by one type of derivative, $\frac{\partial R_{p}\left(i_{w}\right)}{\partial q_{I}\left(j_{w}, j_{s}\right)}$.

This time-stepping procedure clearly increases the solution time. The computation time is mainly spent on evaluating the potentials $\Phi_{D}\left(j_{w}, j_{s}, i_{w}, i_{s}\right)$ at each time step. Nevertheless, the solution procedure remains very efficient, as only a relatively small number of time steps need to be taken for a typical problem in which the well rates vary relatively slowly. With this formulation, the system of equations can be formed to satisfy either a specified bottomhole pressure or a total rate constraint.

\subsection{Example Cases}

Results using the semianalytical approach have been compared with those of a commercial finite difference numerical simulator (ECLIPSE) for a number of test cases. Extensive results and comparisons were presented by Wolfsteiner et al. (2000), Valvatne et al. (2000, 
2001, 2003), Serve (2002) and Fukagawa (2002). Here we present a few representative results that demonstrate the capabilities of the semianalytical modeling procedure.

\subsubsection{Offshore Nigeria Reservoir Model}

This data set, representing a portion of a field located offshore Nigeria, was provided by ChevronTexaco. The fine scale geostatistical model is of dimension $153 \times 72 \times 40$ cells, for a total of approximately 440,000 cells. Of the 440,000 cells, 26,691 cells were used for our single-well study. This field is a sand/shale reservoir with permeabilities and anisotropy ratio $\left(k_{\downarrow} / k_{h}\right)$ that vary with position in the reservoir. The detailed property data for this case is given in Fukagawa (2002).

Applying the tuning procedure for the power averaging coefficients gives $\omega_{x}=\omega_{y}=0.8$ and $\omega_{z}=-0.1$ for this model. These values provide close agreement between semianalytical calculations and finite difference calculations for vertical and horizontal wells that are aligned with the finite difference grid (in which case the finite difference results can be considered to be the reference solution). We now use these $\omega$ values for the simulation of a dual-lateral well. The upper lateral is aligned with the $y$-direction and the lower lateral is parallel to the $x$-direction (as shown in Fig. 4-3, upper left). The total production rate is 5,000 $\mathrm{STB} / \mathrm{d}$. The agreement in pressure between the semianalytical (designated $s-\mathbf{k}^{*}$ ) and finite difference results is excellent. The inflow profiles show some differences, but the overall level of agreement is quite reasonable. This demonstrates that, once the appropriate $\omega$ (and

thus $\mathbf{k}^{*}$ ) are determined, the $s-\mathbf{k}^{*}$ model can provide reliable results for general well types and trajectories. 

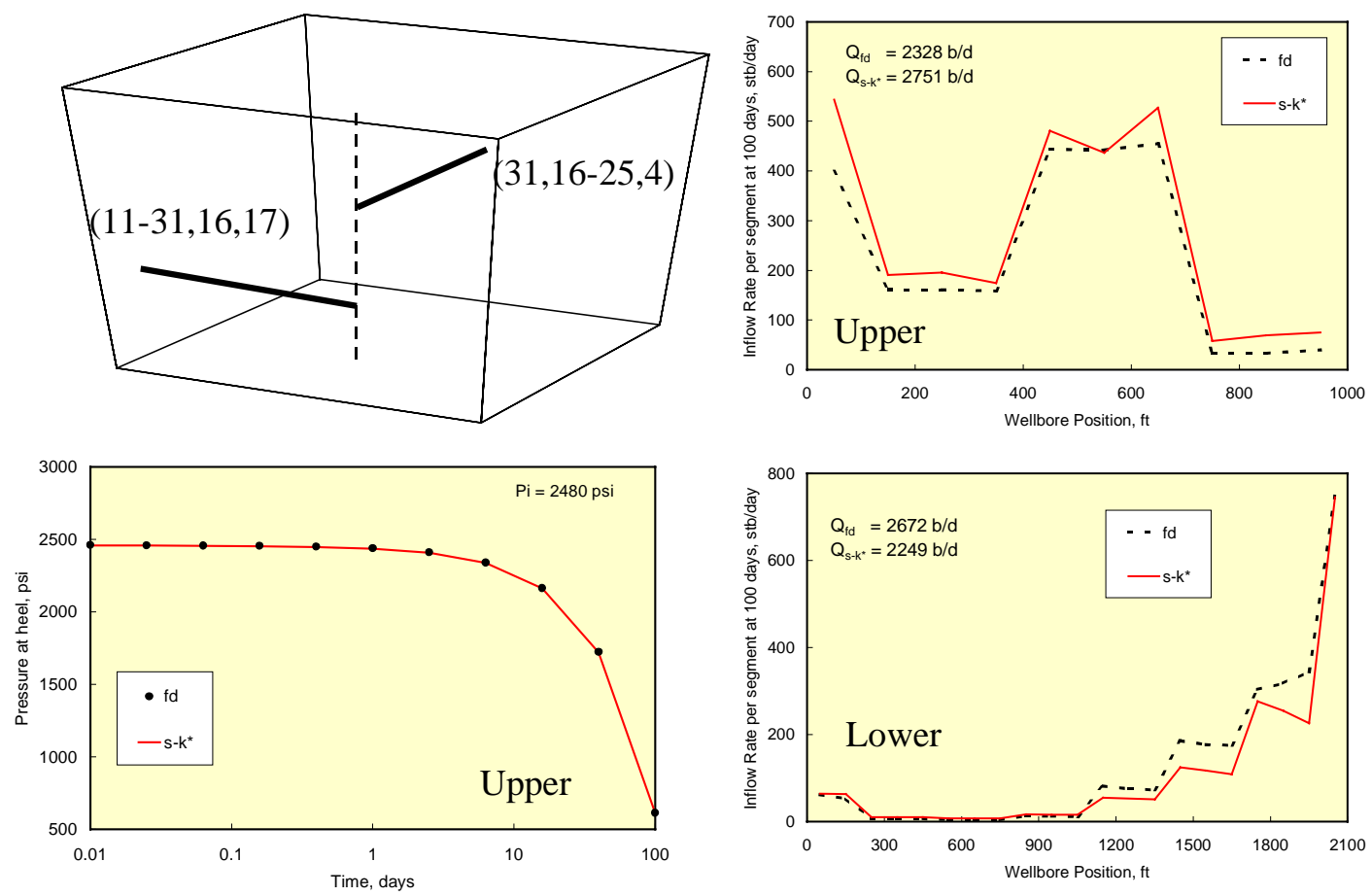

Figure 4-3: Inflow and pressure performance for the dual lateral well case

\subsubsection{Channelized Reservoir Produced with Segmented Well}

This case involves a highly heterogeneous fluvial reservoir, shown in Fig. 4-4 (Mao and Journel, 1999). The permeability distribution was generated using geostatistical techniques, with the channels introduced through use of object-based simulation. The overall mean permeability is $176 \mathrm{md}$, though two distinct populations of permeability are clearly evident. The channels contain sandstone with a mean permeability of about $200 \mathrm{md}$, while the mudstone-dominated background has an average permeability of about $6 \mathrm{md}$. The components of the effective permeability tensor $\mathbf{k}^{*}$, obtained using a flow-based upscaling technique, are $k_{x}^{*}=136, k_{y}^{*}=135, k_{z}^{*}=12$. Average reservoir porosity is 0.17 . The well rate is $30,000 \mathrm{STB} / \mathrm{d}$ and the initial reservoir potential is $3000 \mathrm{psi}$. The total system compressibility is $3 \times 10^{-6} \mathrm{psi}^{-1}$ and the oil density is $60 \mathrm{lb}_{\mathrm{m}} / \mathrm{ft}^{3}$. The reservoir boundaries are sealed (no flow). Further details are given by Valvatne et al. (2003). 
The well location is indicated in Fig. 4-4. The permeability contrast is very large along the well, varying from more than 500 md to about $7 \mathrm{md}$ over a few feet. Inflow profiles computed using ECLIPSE and AdWell (semianalytical solution method) are shown in Fig. 4-5. The ECLIPSE model contained $100 \times 130 \times 30$ blocks. Again, because the well is aligned with the simulation grid, the finite difference results are considered to be reliable in this case. Given the complexity of the permeability field and the approximate nature of the $s$ $\mathbf{k}^{*}$ representation of permeability heterogeneity, the agreement in inflow profiles between the two results is quite satisfactory. The bottomhole pressures for finite difference and semianalytical calculations at a time of 100 days are 2,251 and 2,207 psi, corresponding to an error in predicted drawdown of less than $10 \%$ (average reservoir pressure at 100 days is $2,711 \mathrm{psi})$.

The high flow rates from high permeability channels in this reservoir would likely result in early water or gas breakthrough. From a reservoir management standpoint, it may be desirable to reduce the production from the channel to obtain a more uniform influx. Downhole chokes are now introduced into the model and it is shown how they can be used to flatten the inflow profile. As the choke is closed, higher pressures will occur at the choke inflow (i.e., at the sandface), which in turn results in a lower local drawdown and less inflow from the zone.

For the case under study, we use three inflow zones along the well, with the objective being to reduce inflow from the central section completed in the channel. The settings of the chokes are obtained by trial and error until an "optimized" inflow profile is achieved. Only the central inflow zone required choking. The original and optimized inflow profiles are shown in Fig. 4-6. By imposing a 709 psi pressure drop across the choke, it is possible to reduce the inflow from the channel from 22,748 to $11,622 \mathrm{STB} / \mathrm{d}$. This represents a reduction from $75 \%$ to $39 \%$ of the total inflow. The inflow from zone 3 (see Fig. 4-6 for the exact location of the zones) is still low, representing only $17 \%$ of the total. The permeability in this section, which is less than $10 \mathrm{md}$, is just too low for the inflow to increase to that of the other zones. Nonetheless, there is a clear flattening of the inflow profile along the well, which would be expected to result in a delay of water or gas breakthrough. The convergence of the 
solution is very rapid in this case and was achieved after only five iterations. The solution is in relatively close agreement with the finite difference result (see Valvatne et al., 2003).

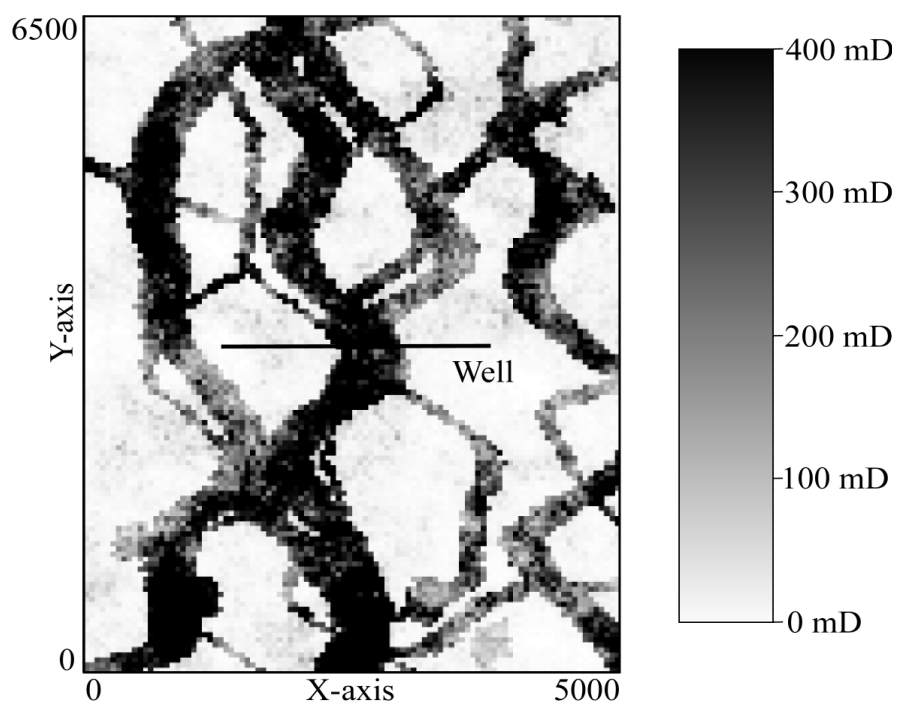

Figure 4-4: Fluvial reservoir permeability field and horizontal well

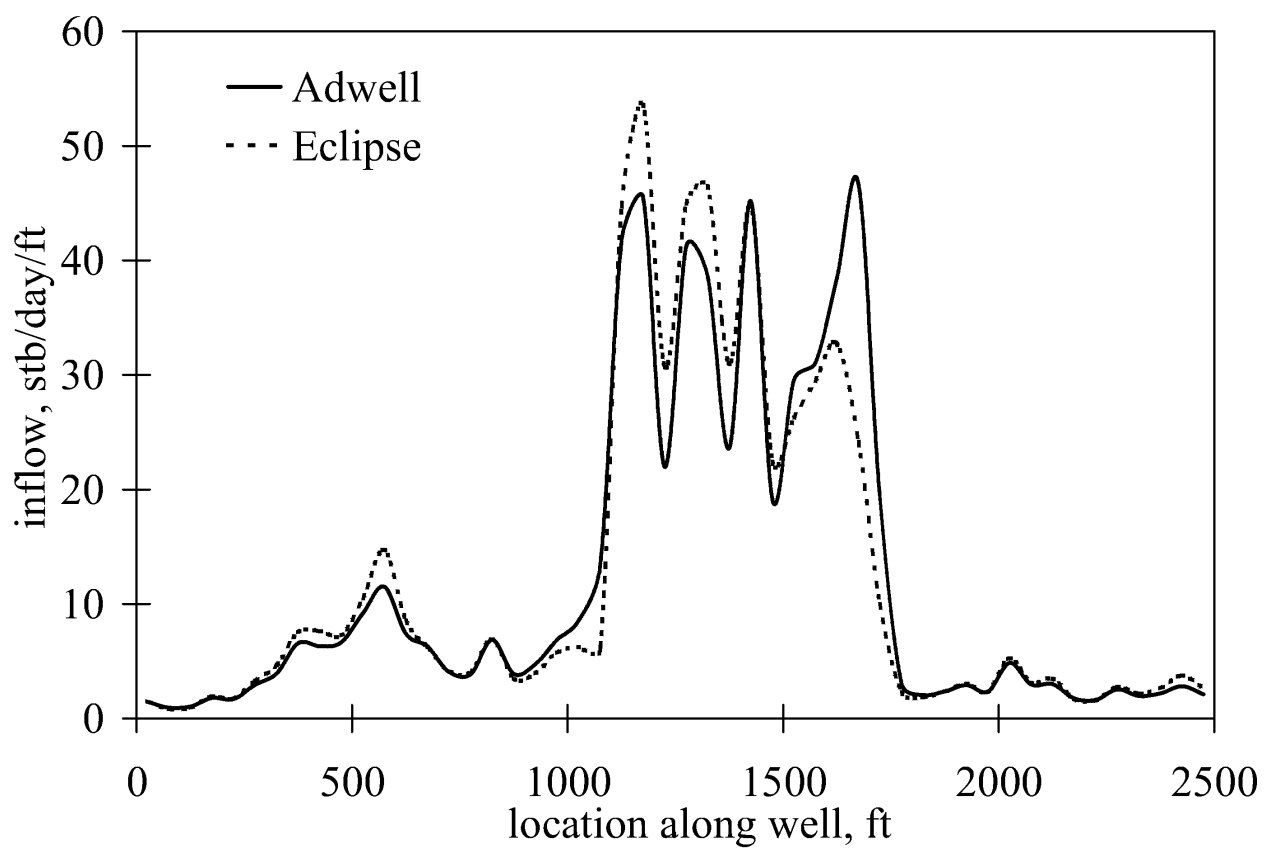

Figure 4-5: Inflow profiles in fluvial reservoir 


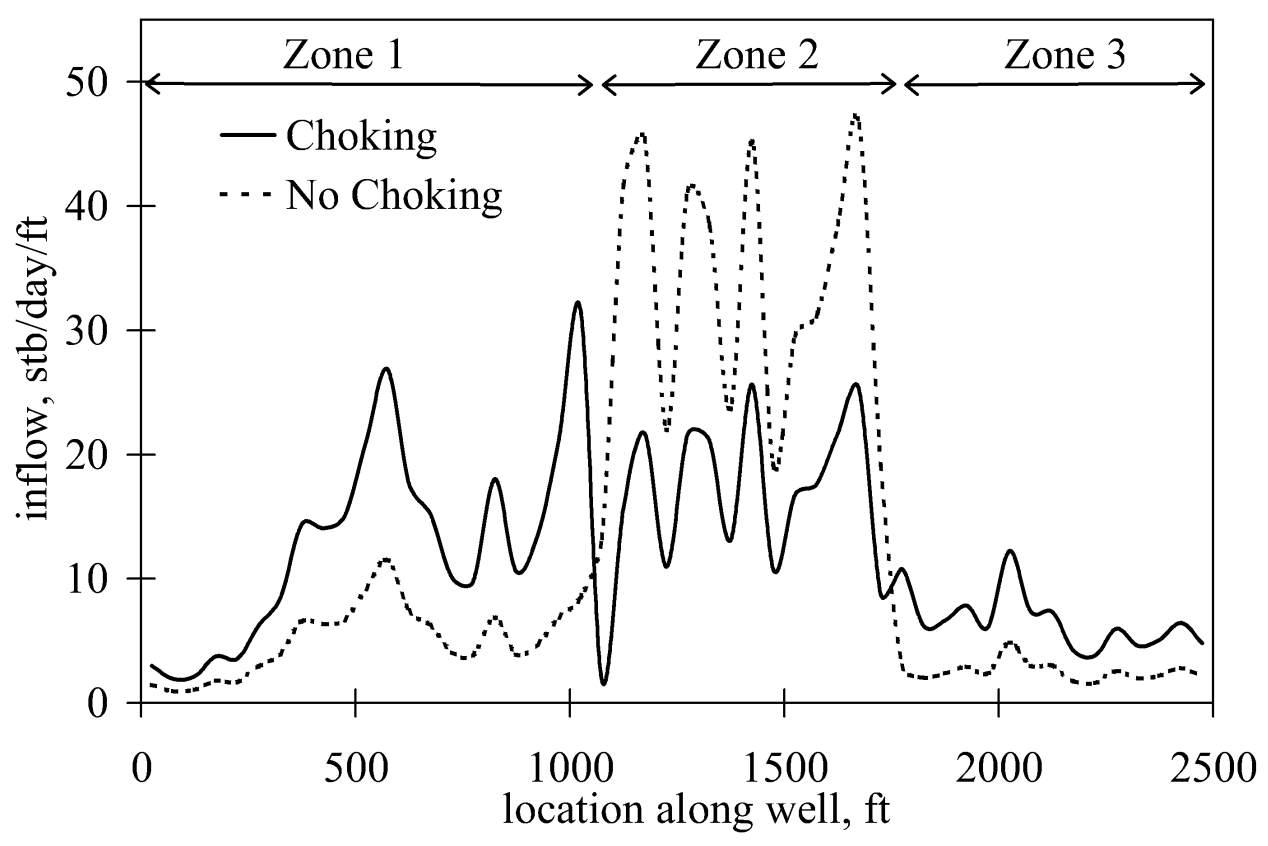

Figure 4-6: Inflow profiles with and without downhole choking

\subsubsection{Fixed Pressure Well}

The next case involves a well operating at fixed bottom hole pressure. For this case, a homogeneous reservoir of dimension $5000 \times 6500 \times 300 \mathrm{ft}$ and permeability $k_{x}=136$, $k_{y}=135, k_{z}=12$ is used. Production is via a $2350 \mathrm{ft}$ horizontal well, centered in the reservoir. The well is modeled with 34 inflow segments and is specified to produce at a fixed bottom hole pressure of 2500 psia. A choke, set to $70 \%$ open, is also included, as are the effects of wellbore hydraulics. The total well rate and inflow distribution determined by the semianalytical model are compared to the finite difference calculations at a time of 100 days, when the reservoir has reached pseudo-steady state. As is apparent from Figs. 4-7 and 4-8, the matches for both the well rate and the inflow profile are quite close, indicating that the fixed pressure well specification is implemented correctly. The slight deviation in well rate at early time (Fig. 4-7) may be due to inaccuracies in the treatment of the early transient in the finite difference simulator. 


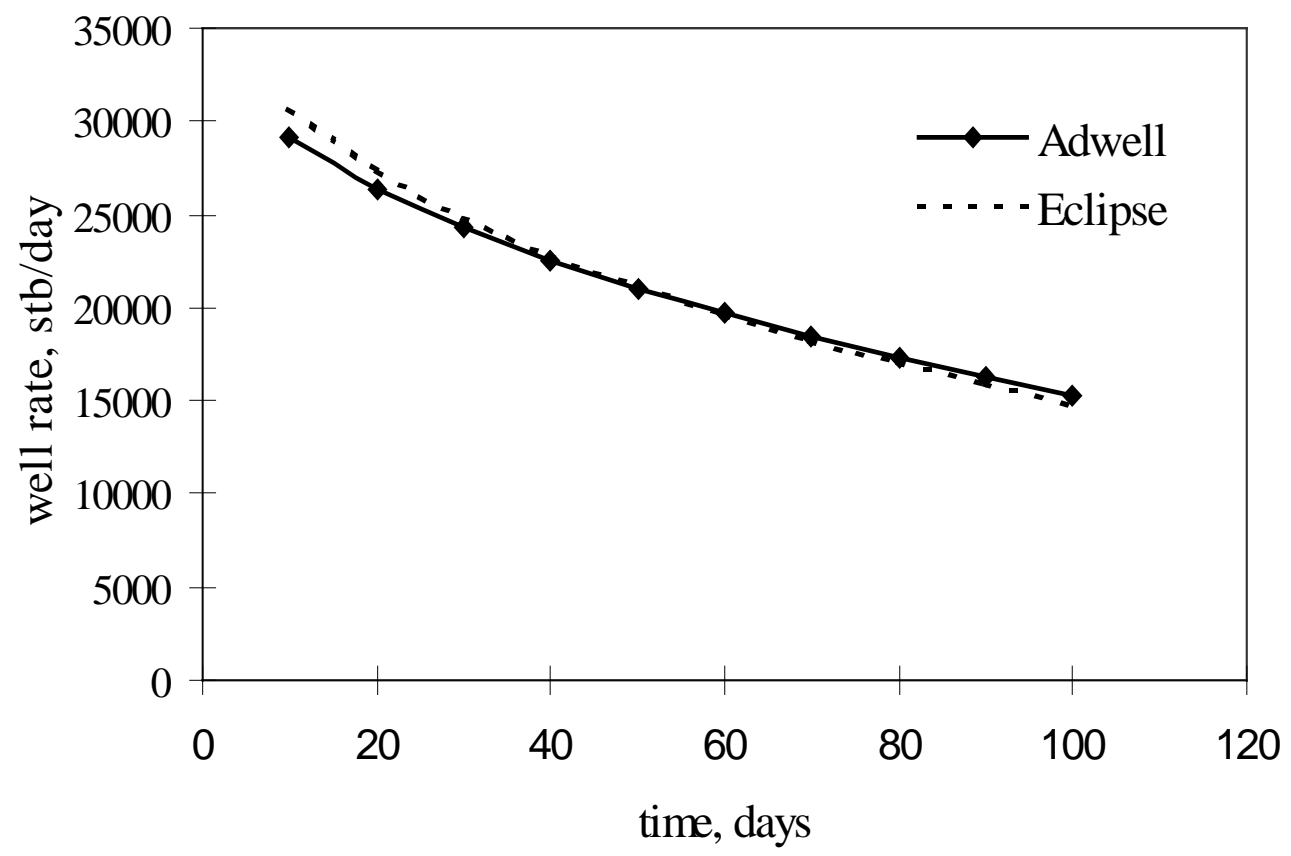

Figure 4-7: Comparison with finite difference simulator: total rate with fixed BHP

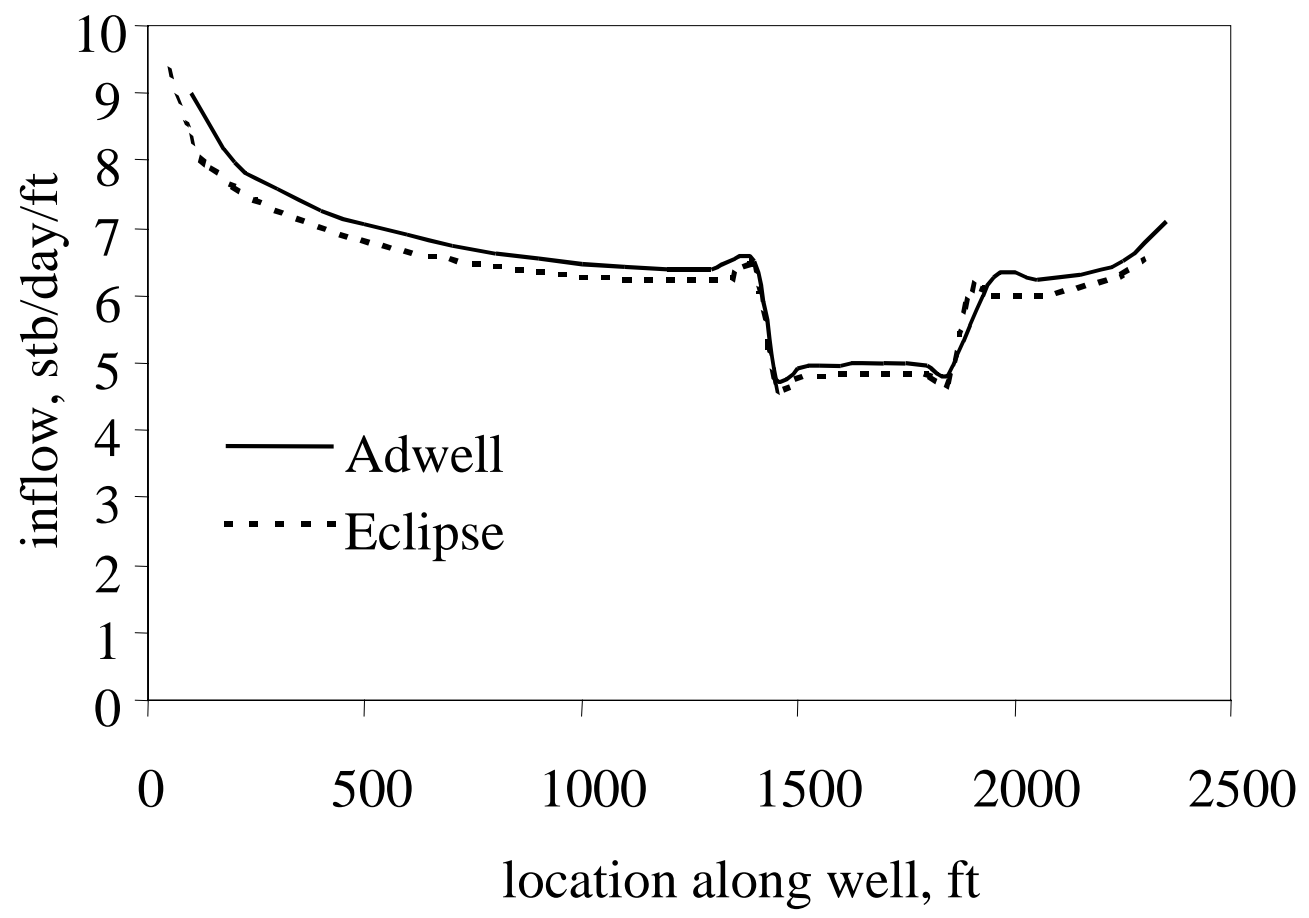

Figure 4-8: Comparison with finite difference simulator: inflow profile at fixed BHP 


\subsection{Procedure for the Calculation of Well Index}

We now present a general framework for the computation of well indices for an arbitrary coarse grid (see Wolfsteiner et al., 2003, for full details). This procedure is general and can also be applied to unstructured coarse grids. We are interested in obtaining appropriate well indices for each well block $i$ given an arbitrary target grid and an arbitrary well trajectory. In the following discussion, the subscript $i$ denotes a well block quantity (finite difference simulation), while $j$ denotes a quantity for a well segment (semianalytical model).

We consider a local well driven flow problem as depicted on the top of Fig. 4-9. The local well domain is selected by the user and would typically correspond to the drainage volume for a single or multiple wells. Due to the nature of the semianalytical reference solution, the selected well domain must be box shaped. The boundary conditions can be constant potential or no flow, as appropriate. We found that the computed well index is not very sensitive to these boundary conditions, as long as they are consistent between the reference and target grid solutions. The specification of the total well flow rate and no flow conditions for all boundaries except the bottom, where constant pressure is specified, allows for an efficient solution because the well will reach steady-state (thus avoiding transient calculations).

The local problem is solved first, using the semianalytical method described above. For a heterogeneous problem, the semianalytical tool uses the geostatistical fine grid permeability field, represented in terms of $s_{j}$ and $\mathbf{k}^{*}$ as explained above. In either case the solution provides the well pressure $p_{j}^{w}$ and the flow rate $q_{j}^{w}$ for each segment $j$. These results are reference quantities that will be used to compute the appropriate well indices for the simulation grid. The same local domain and boundary conditions as used in the semianalytical solution are applied to obtain the numerical solution for block pressures on the target grid. For a heterogeneous problem, the fine grid permeability information must first be upscaled to the target grid. We then solve the single phase steady state pressure equation on the target grid,

$$
\nabla \cdot(\mathbf{k} \nabla \boldsymbol{\Phi})=q
$$




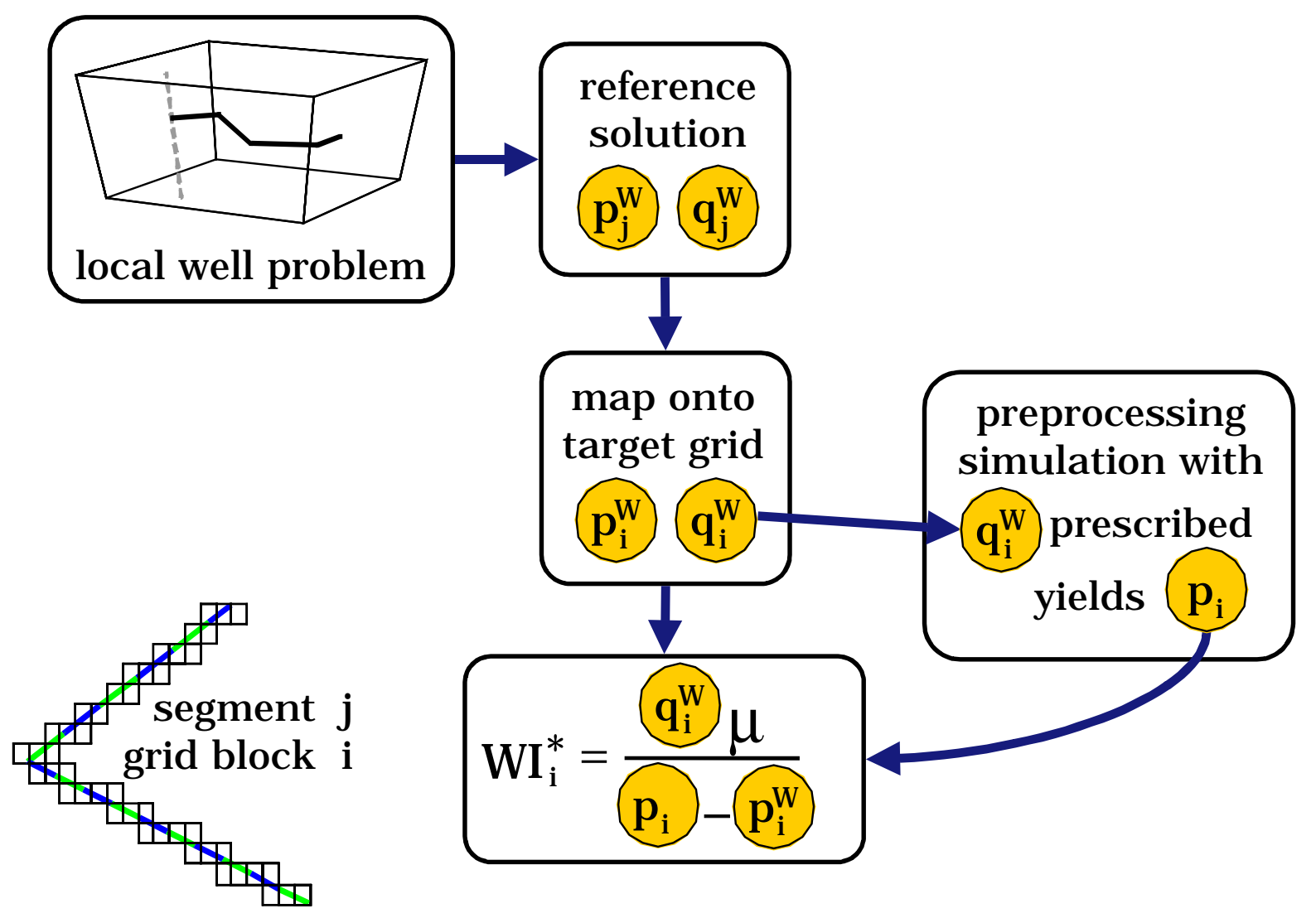

Figure 4-9: Flowchart illustrating computation of accurate well index

where the source term $q$ is nonzero in each block $i$ in which a well is completed, i.e., we prescribe sources $q_{i}^{w}$ for each block intersected by the well (rather than specifying the total well rate as before). As a segment $j$ is generally not contained by exactly one block $i$ (as indicated in Fig. 4-9), we need to apply a mapping procedure to compute the intersections of the well with the grid. Our intersection algorithm is quite general (Arvo, 1991) in that any polyhedral unstructured grid can be considered as long as the grid cells are convex. With this information, the rates $q_{i}^{w}$ are determined from the $q_{j}^{w}$ based on the fractional length $l_{i j}$ of the well segment $j$ in block $i$ :

$$
q_{i}^{w}=\sum_{j=1}^{j=j_{N}} l_{i j} q_{j}^{w}
$$


where the summation over all $j_{N}$ segments in the domain accounts for the possibility that multiple wells may intersect block $i$.

The wellbore pressure corresponding to block $i, p_{i}{ }^{w}$, which will be needed for the final calculation of the well index, is simply computed from the reference $p_{j}^{w}$ corrected by the hydrostatic head between the level of the segment midpoint and the block midpoint. The prescribed block rates $q_{i}^{w}$ act as simple source terms in the simulator. It is important to note that the well model of the target simulator does not enter this procedure, as this is the quantity we seek to compute. Following the finite difference solution of Eq. 4-35, the pressure of each well block $p_{i}$ is known.

The correct well index for block $i$ can now be computed from the semianalytical (reference) well pressure and rate information, interpolated onto the well blocks using Eq. 436 and the block pressures obtained from the target simulator:

$$
W I_{i}^{*}=\frac{q_{i}^{w} \mu}{p_{i}-p_{i}^{w}}
$$

The * superscript is used to distinguish this well index from the default well index $W I^{d}$ computed by the standard Peaceman (1983) formula (based on the grid block $\mathbf{k}^{*}$ ).

Use of the $W I_{i}^{*}$ computed from Eq. 4-37 will provide finite difference results for $p_{i}^{w}$ consistent with the $p_{j}^{w}$ from the semianalytical solution for the specified reference problem. Similarly, if the finite difference simulator is now run with $p_{i}^{w}$ specified, the inflow profiles of the two solutions (provided the $q_{j}^{w}$ are integrated along the well in a manner consistent with Eq. 4-36) should match. However, there is no guarantee that these $W I_{i}^{*}$ are appropriate for use in all flow problems. This is equivalent to noting that the $W I_{i}^{*}$ computed from the procedure described above will vary somewhat if the boundary conditions are varied. We have, however, observed this variation to be very slight, indicating that the well indices computed using our procedure can be expected to display a high level of robustness. In the case of heterogeneous systems, our method for computing the well index includes an 
upscaling component, since the effects of near well, fine scale heterogeneity are included in the coarse grid $W I_{i}^{*}$.

Finally, it should be noted that our overall framework is not restricted to the type of reference solution applied in this work. In fact, any other semianalytical or finite difference reference solution is suitable as long as it can provide the required source terms $q_{i}^{w}$ and local wellbore pressures $p_{i}^{w}$. Fine grid finite difference models that honor the well trajectory and share common boundaries with the coarse grid, such as the multiblock method (Jenny et al., 2002), may be applied to provide a reference solution for complex wells in heterogeneous permeability fields.

\subsection{Calculation of Well Index for a Multiblock Grid}

For this example we will use a complex grid constructed to model a pinchout (three geologic layers on the right side of the model collapsing to two layers on the left side). The multiblock grid shown in Fig. 4-10 is comprised of nine blocks, each of which has $8 \times 8 \times 8$ cells. The physical dimensions of the system are $500 \times 250 \times 250 \mathrm{~m}$. The permeability field is homogenous and isotropic $(k=100 \mathrm{md})$. The first step in our procedure (see Fig. 4-9) is to obtain the semianalytical reference solution. In this case, we use a resolution of 15 segments to obtain the reference well inflow and pressure profiles for a well specified to produce $Q=$ $10,000 \mathrm{STB} / \mathrm{d}$.

We next perform an intersection of the well trajectory with the grid as illustrated in Fig. 4-11. This enables us to map the reference flow rates onto the target grid, where they are used as source terms in the finite difference calculations. The pressure field for the finite difference solution on the multiblock grid can then be computed. The appropriate well index can now be determined and then used in the finite difference simulator. Fig. 4-12 compares steady state inflow profiles and well segment pressures along the well trajectory computed using the finite difference method with $W I^{*}$ (points) and the reference semianalytical solution (curves). As expected, the finite difference solution with $W I^{*}$ traces the reference result accurately. This demonstrates the applicability of the method for complex grids and general well trajectories. Note that we do not compare the multiblock grid results to any simulations 
using a default well index in this case because it is not clear how to compute such well indices for the grid of Fig. 4-10 using standard procedures.

Several other examples involving the calculation of $W I^{*}$ are presented in Wolfsteiner et al. (2003). These examples demonstrate the ability of the method to account for general intersections between the wellbore and the well block and to determine $W I^{*}$ for complex multilateral wells. The ability of the overall procedure to account for the effects of fine-scale permeability heterogeneity on $\mathrm{WI}^{*}$ is also illustrated.

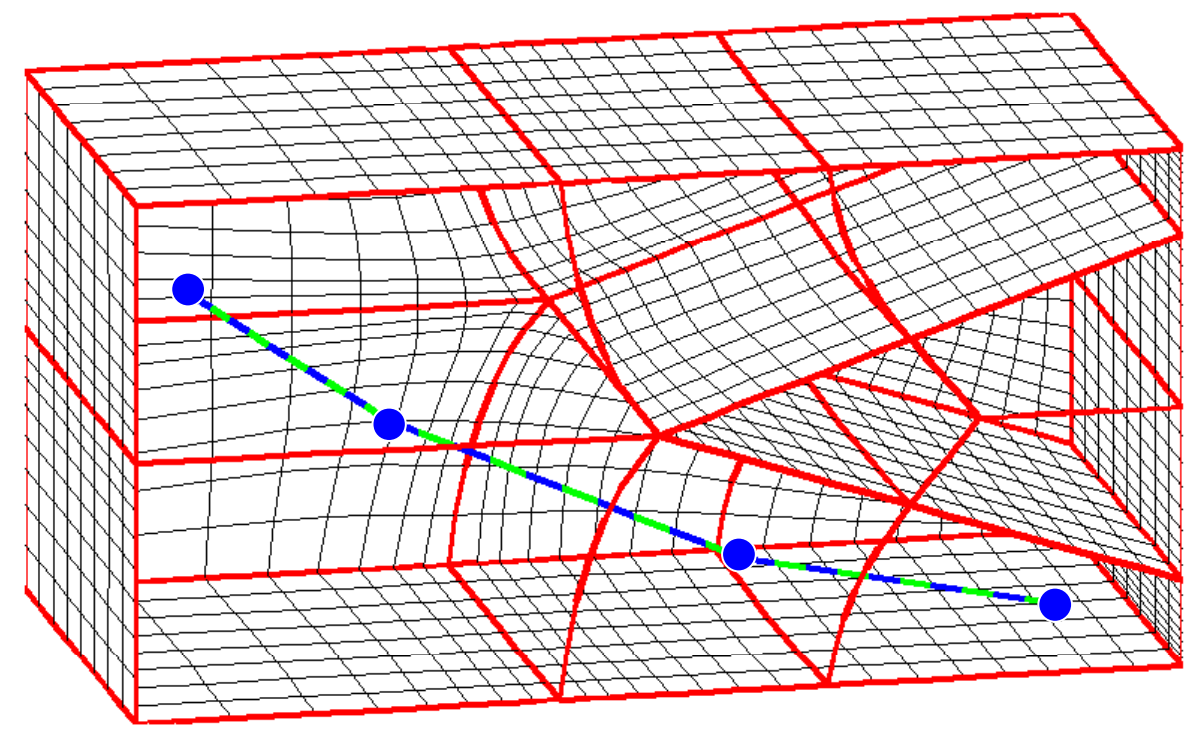

Figure 4-10: Multiblock grid with deviated well 


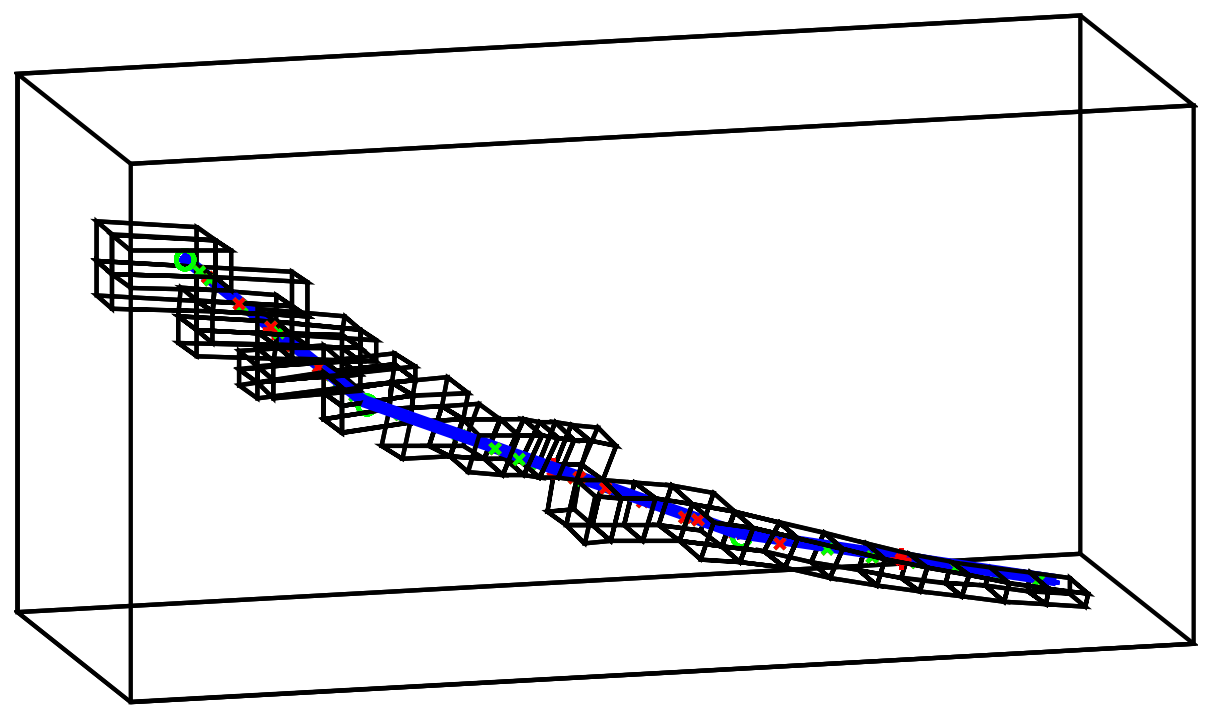

Figure 4-11: Intersection of deviated well with grid for the determination of well blocks

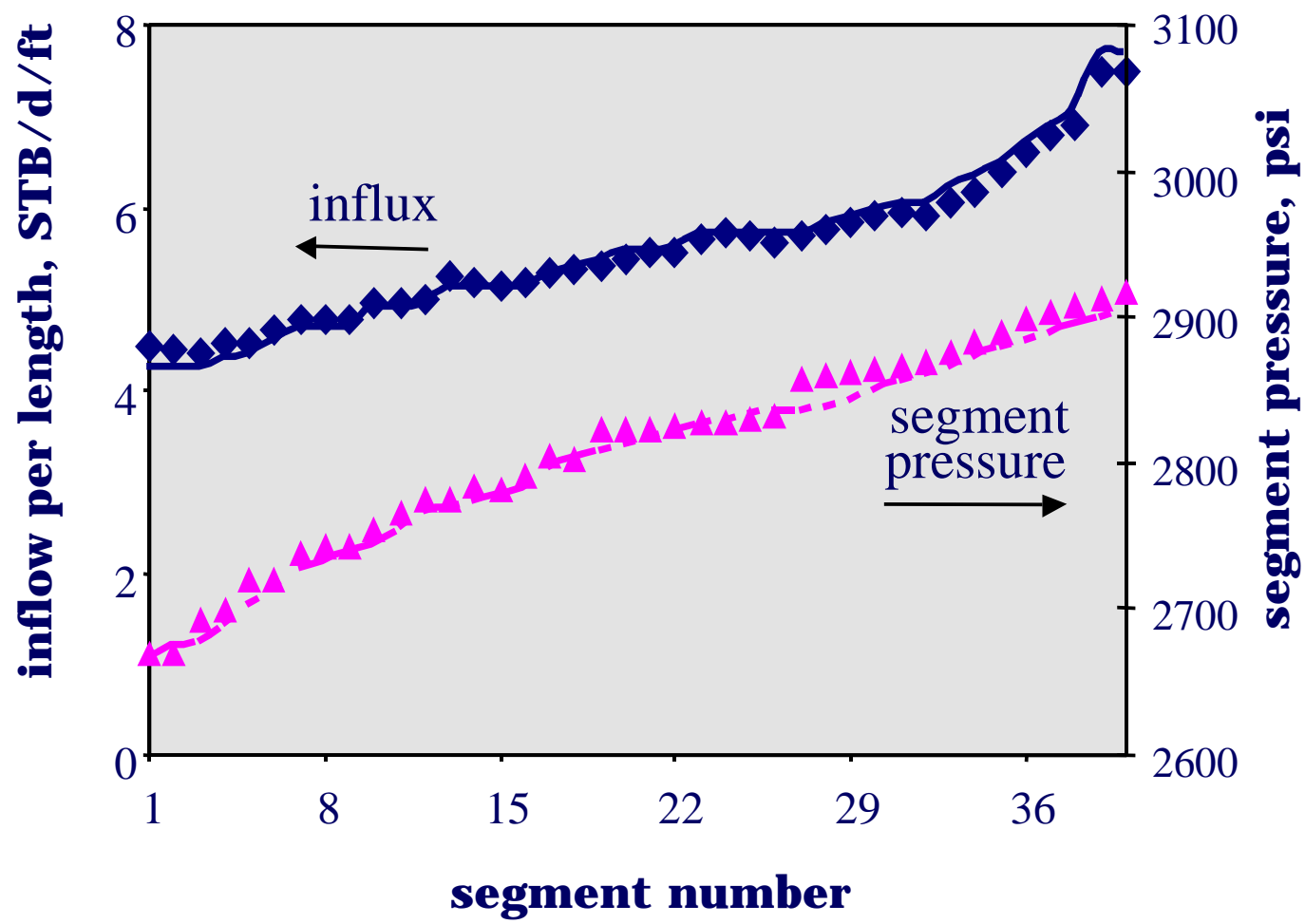

Figure 4-12: Inflow and pressure profiles (curves represent semianalytical reference solution; points represent finite difference results using $W I^{*}$ ) 


\subsection{Conclusions}

The following main conclusions can be drawn from this work:

- Semianalytical solution procedures based on Green's functions are well suited for the modeling of nonconventional wells producing under single-phase flow. These techniques were developed and applied for both oil and gas flows and for wells producing under rate and bottomhole pressure specifications.

- The effects of heterogeneity, wellbore pressure losses and downhole chokes were successfully incorporated into the overall formulation. Comparison to reference finite difference results demonstrated the degree of accuracy that can be achieved using the semianalytical solution technique.

- The $s-\mathbf{k}^{*}$ permeability representation was extended for application to real field problems where anisotropy is present in the fine-scale permeability field. A tuning procedure was presented which provides power averaging coefficients that result in close agreement between wellbore pressure computed semianalytically and that computed via finite difference.

- The semianalytical solution technique was applied to determine the well index for use in finite difference simulators. The procedure was shown to be applicable for complex wells modeled on arbitrary simulation grids.

\subsection{Nomenclature}

$B$ formation volume factor, RB/STB

C unit system conversion factor

$c_{t} \quad$ total compressibility, $1 / \mathrm{psi}$

$D$ diameter, $\mathrm{ft}$

$f \quad$ Fanning friction factor

$g$ acceleration due to gravity, $\mathrm{lb}_{\mathrm{f}} \mathrm{ft} / \mathrm{sec}^{2}$

$g_{c} \quad$ gravitational conversion constant, $32.2 \mathrm{lb} / \mathrm{lb}_{\mathrm{f}} \cdot \mathrm{ft} / \mathrm{sec}^{2}$

$h \quad$ height from datum depth, $\mathrm{ft}$

k reservoir permeability, md 


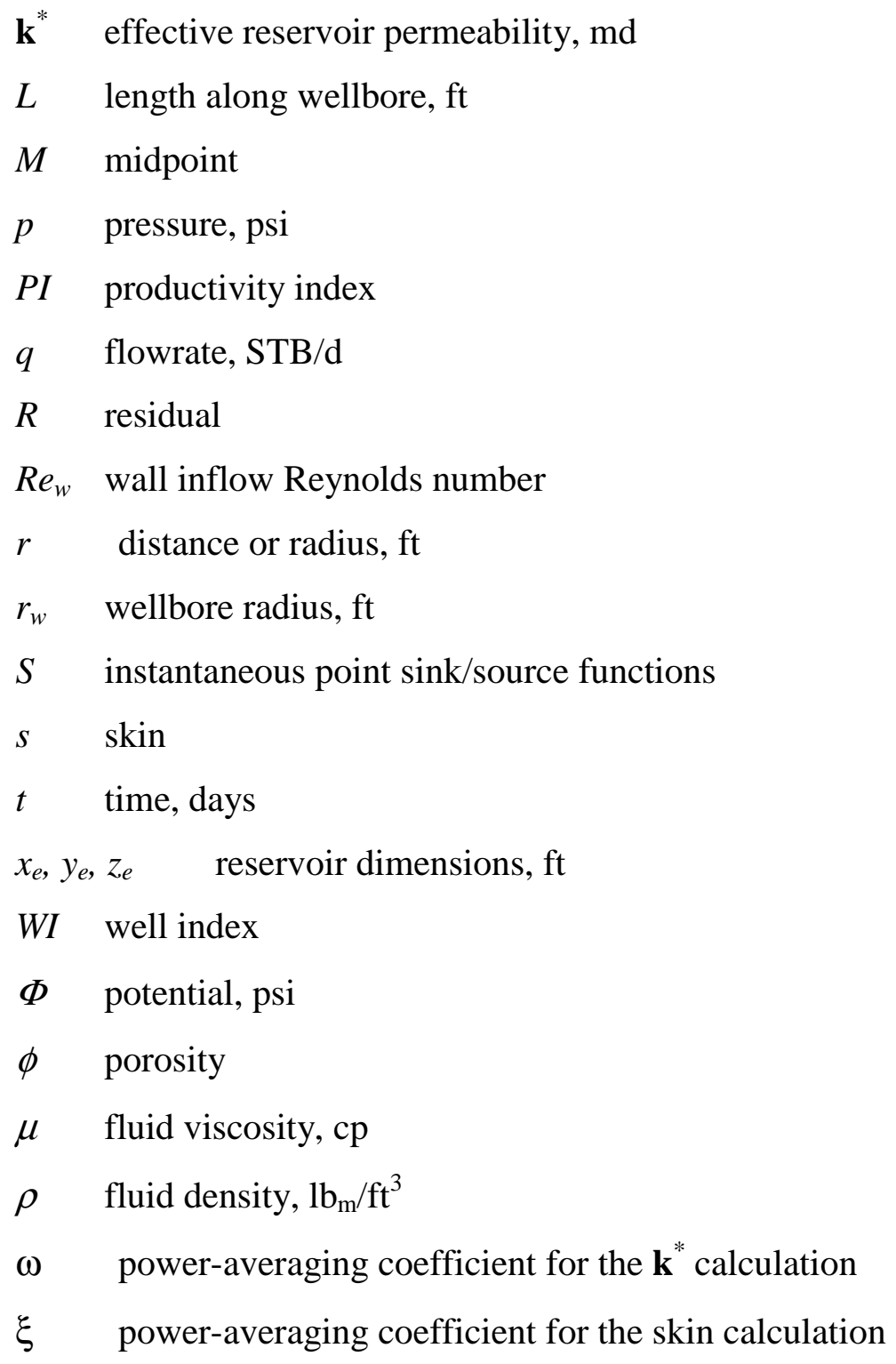

\section{Superscripts}

* effective

, transformed

w well or well block

\section{Subscripts}

a altered zone or accelerational

c choke

D dimensionless 


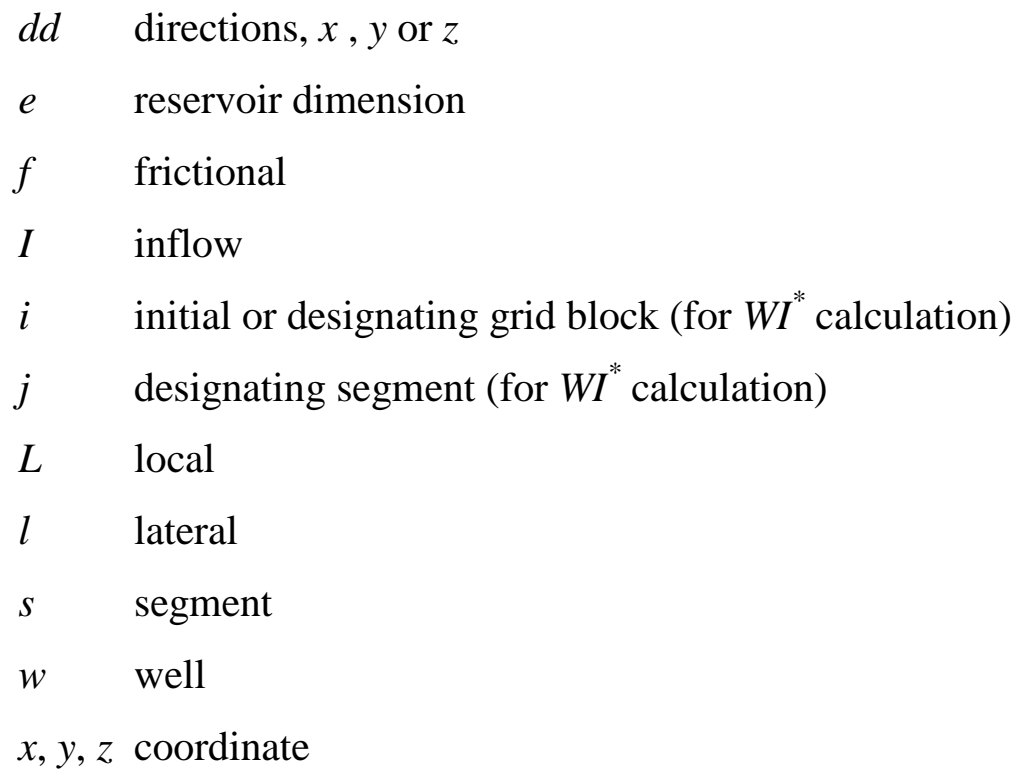




\subsection{References}

Arvo, J.: Graphic Gems, vol. II, Academic Press, Boston, MA, 1991.

Babu, D.K. and Odeh, A.S.: "Productivity of a Horizontal Well," SPERE, 417-21, Nov. 1989.

Basquet, R., Alabert, F.G., Caltagirone, J.P. and Batsale, J.C.: "A Semi-Analytical Approach for Productivity Evaluation of Wells with Complex Geometry in Multilayered Reservoirs," paper SPE 49232 presented at the SPE Annual Technical Conference and Exhibition, New Orleans, Sept. 27-30, 1998.

Besson, J.: "Performance of Slanted and Horizontal Wells on an Anisotropic Medium," paper SPE 20965 presented at the Europec, The Hague, Netherlands, Oct. 22-24, 1990.

Brigham, W.E.: "Discussion of Productivity of a Horizontal Well," SPERE, 255-255, May 1990.

Clonts, M.D. and Ramey, H.J., Jr: "Pressure Transient Analysis for Wells with Horizontal Drainholes," paper SPE 15116 presented at the SPE California Regional Meeting, Oakland, Apr. 2-4, 1986.

Daviau, F., Mouronval, G., Bourdarot, G. and Curutchet, P.: "Pressure Analysis for Horizontal Wells," paper SPE 14251 presented at the SPE Annual Technical Conference and Exhibition, Las Vegas, Sept. 22-25, 1985.

Deutsch, C.V.: "Calculating Effective Absolute Permeability in Sandstone/Shale Sequences," SPEFE, 343-348, Sept. 1989.

Durlofsky, L. J.: “An Approximate Model for Well Productivity in Heterogeneous Porous Media," Mathematical Geology, 32, 421-438, 2000.

Economides, M.J., Brand, C.W. and Frick, T.P.: "Well Configurations in Anisotropic Reservoirs," SPEFE, 257-262, Dec. 1996.

Fukagawa, H.: "Semianalytical Modeling of Nonconventional Well Performance in Realistic Reservoirs," Master's report, Stanford University, 2002.

Goode, P.A. and Thambynayagam, R.K.M.: "Pressure Drawdown and Buildup Analysis of Horizontal Wells in Anisotropic Media," SPEFE, 683-97, Dec. 1987.

Haaland, S.E.: "Simple and Explicit Formula for the Friction Factor in Turbulent Pipe Flow Including Natural Gas Pipelines," IFAG B-131, Technical Report, Division of Aero- and Gas Dynamics, The Norwegian Institute of Technology, Norway, 1981.

Hawkins, M.F.: “A Note on the Skin Effect,” Trans. AIME, 356-357, 1956.

Jenny, P., Wolfsteiner, C., Lee, S.H., Durlofsky, L.J.: "Modeling Flow in Geometrically Complex Reservoirs Using Hexahedral Multiblock Grids,” SPEJ, 149-157, June 2002. 
Journel A.G., Deutsch, C.V., and Desbarats, A.J.: "Power Averaging for Block Effective Permeability," paper SPE 15128 presented at the California Regional Meeting, Oakland, CA, April 2-4, 1986.

Kuchuk, F.J., Goode, P.A., Brice, B.W., Sherrared, D.W. and Thambynayagam, R.K.M.: "Pressure Transient Analysis and Inflow Performance for Horizontal Wells," paper SPE 18300 presented at the SPE Annual Technical Conference and Exhibition, Houston, Oct. 2$5,1988$.

Lee, S.H. and Milliken, W.J.: "The Productivity Index of an Inclined Well in FiniteDifference Reservoir Simulation," paper SPE 25247 presented at the SPE Symposium on Reservoir Simulation, New Orleans, Feb. 28-Mar. 3, 1993.

Maizeret, P.D.: "Well Indices for Nonconventional Wells," Master's report, Stanford University, 1996.

Mao, S. and Journel. A.G.: "Generation of a Reference Petrophysical / Seismic Data Set: The Stanford IV Reservoir, Stanford Center for Reservoir Forecasting Report, May 1999.

Ouyang, L.-B.: "Single Phase and Multiphase Fluid Flow in Horizontal Wells," PhD thesis, Stanford University, 1998.

Ouyang, L.B., Arbabi, S., and Aziz, K.: "A Single-Phase Wellbore-Flow Model for Horizontal, Vertical, and Slanted Wells," SPEJ, 124-133, June 1998.

Ouyang, L-B. and Aziz, K.: “A General Single-Phase Wellbore/Reservoir Coupling Model for Multilateral Wells," SPERE\&E, 327-335, Aug. 2001.

Ozkan, E., Raghavan, R. and Joshi, S.D.: "Horizontal Well Pressure Analysis," SPEFE, 56775, Dec. 1989.

Peaceman, D.W.: "Interpretation of Well-Block Pressure in Numerical Reservoir Simulation," SPEJ, 531-543, June 1983.

Sachdeva, R., Schmidt, Z., Brill, J.P., and Blais, R.M.: "Two-phase Flow through Chokes," paper SPE 15657 presented at the SPE Annual Technical Conference and Exhibition, New Orleans, Oct. 5-8, 1986.

Schlumberger GeoQuest Inc.: ECLIPSE Reservoir Simulator 99A, E100, 1999.

Serve, J.: "Enhanced Semianalytical Modeling of Complex Well Configurations," Master's report, Stanford University, 2002.

Valvatne, P.H.: "A Framework for Modeling Complex Well Configurations," Master's report, Stanford University, 2000.

Valvatne, P.H., Durlofsky, L.J., and Aziz, K.: "Semianalytical Modeling of the Performance of Intelligent Well Completions," paper SPE 66368 presented at the SPE Reservoir Simulation Symposium, Houston, Feb. 11-14, 2001. 
Valvatne, P.H., Serve, J., Durlofsky, L.J., and Aziz, K.: "Efficient Modeling of Nonconventional Wells with Downhole Inflow Control Devices," J. Pet. Sci. Eng., 39, 99116, 2003.

Wolfsteiner, C., Durlofsky, L.J., and Aziz, K.: "Approximate Model for Productivity of Nonconventional Wells in Heterogeneous Reservoirs," SPEJ, 218-226, June 2000.

Wolfsteiner, C., Durlofsky, L.J., and Aziz, K.: "Calculation of Well Index for Nonconventional Wells on Arbitrary Grids," Comp. Geosciences, 7, 61-82, 2003. 


\section{Modeling of Multiphase Flow in Wellbores}

\subsection{Introduction}

Multiphase flow effects in wellbores and pipes can have a strong impact on the performance of reservoirs and surface facilities. In the case of horizontal or multilateral wells, for example, pressure losses in the well can lead to a loss of production at the toe or overproduction at the heel. In order to model and thereby optimize the performance of wells or reservoirs coupled to surface facilities, accurate multiphase pipeflow models must be incorporated into reservoir simulators.

Within the context of petroleum engineering, the three types of pipeflow models most commonly used are empirical correlations, homogeneous models and mechanistic models. Empirical correlations are based on the curve fitting of experimental data and their applicability is generally limited to the range of variables explored in the experiments. These correlations can be either specific for each flow pattern or can be flow pattern independent. Homogeneous models assume that the fluid properties can be represented by mixture properties and single-phase flow techniques can be applied to the mixture. These models can also allow slip between the phases and this requires a number of empirical parameters. Homogeneous models with slip are called drift-flux models.

Mechanistic models are in general the most accurate as they introduce models based on the detailed physics of each of the different flow patterns. From a reservoir simulation perspective, however, mechanistic models can cause difficulties because they may display discontinuities in pressure drop and holdup at some flow pattern transitions. Such discontinuities can give rise to convergence problems within the simulator. One approach to avoid these convergence issues is to introduce smoothing at transitions. An alternative approach is to apply a homogeneous pipeflow model. The drift-flux model is in fact a simple mechanistic model for intermittent flows, and it is used within general mechanistic models when the flow pattern is predicted to be bubble or slug.

Homogeneous models have the advantages of being relatively simple, continuous and differentiable. As a result, they are well suited for use in reservoir simulators. The simplest homogeneous models, which neglect slip between the fluid phases (i.e., the fluid phases all 
move at the same velocity), are not appropriate for use in reservoir simulators because they fail to capture the complex relationship between the in situ volume fraction and the input volume fraction. Drift-flux models, by contrast, are a good choice for use in reservoir simulators as they do account for the slip between the fluid phases. Drift-flux models are additionally capable of modeling counter-current flow, which allows the heavy and light phases to move in opposite directions when the overall flow velocity is small or when the well is shut in (Hasan et al., 1994). For this reason, the drift-flux model is used in a number of reservoir simulators; e.g., in the multi-segment well model in the ECLIPSE black oil and compositional reservoir simulators (Holmes et al., 1998, Schlumberger GeoQuest, 2001).

Drift-flux models require a number of empirical parameters. Most of the parameters used in current simulators were determined from experiments in small diameter ( 2 inch or less) vertical pipes. These parameters may not be directly applicable to wellbore flows, however, as the flow mechanisms in small pipes can differ qualitatively from those in large pipes (Jepson and Taylor, 1993; Abduvayt et al., 2003). It is therefore important that the drift-flux parameters be determined for wellbores and pipes of sizes of practical interest.

The overall goal of our work in this area is the development of optimized drift-flux models for use in reservoir simulators. In order to accomplish this, extensive experimental and modeling efforts were initiated. The experimental work, reported in detail by Oddie et al. (2003), entailed large-diameter (6 inch) pipeflow experiments performed at a variety of phase flow rates and pipe inclinations. In our current study, these unique experimental data will be used to assess existing drift-flux models and to determine drift-flux parameters for steady state two-phase flows of water-gas and oil-water and the three-phase flow of oil-water-gas in large-diameter pipes or wellbores. The parameters are determined using an optimization technique that minimizes the difference between experimental and model predictions. It is shown that existing default parameters are suboptimal in many cases. Models to accurately represent the effect of pipe inclination are also presented.

The basic drift-flux model was first proposed by Zuber and Findlay (1965). It has since been refined by many researchers (e.g., Nassos and Bankoff, 1967; Wallis, 1969; Ishii, 1977; Hasan and Kabir, 1988a, b, 1999; Ansari et al., 1994) and has been widely used for modeling both liquid-gas and oil-water pipeflow (Flores et al., 1998; Petalas and Aziz, 2000). The 
drift-flux model applies two basic parameters: the profile parameter $C_{0}$ and drift velocity $V_{d}$. Using these parameters (which in turn depend on the system variables), in situ phase volume fractions (holdup) can be calculated from the phase flow rates.

Although drift-flux models are commonly used to represent two-phase flows, comprehensive three-phase flow models are lacking. One treatment for three-phase flow is to combine oil and water into a single 'liquid' phase and to then model the system as a twophase liquid-gas flow. In this treatment, the slip between oil and water is ignored and a homogeneous mixture is assumed for the liquid phase. Some studies indicate that this simple treatment can lead to significant errors in phase holdup predictions (Taitel et al., 1995; Fairhurst and Rarret, 1997), while other observations suggest that this approach is valid (Ozon et al., 1987; Danielson et al., 2000). In this work, we will use our experimental data and model to clearly quantify the range of validity of this approach.

An alternate two-stage technique was proposed in ECLIPSE to model three-phase flow in wellbores (Holmes et al., 1998; Schlumberger GeoQuest, 2001). This approach uses twophase liquid-gas and oil-water flow models. In the first stage, oil-water-gas flow is treated as a liquid-gas flow with flow-weighted average properties for the liquid phase. The liquid-gas drift-flux model is applied to determine the gas and liquid holdups. In the second stage, the oil-water drift-flux model is applied to compute the oil and water holdups within the liquid phase. This idealized approach ignores the effect of the third phase on the two-phase flow models. Nevertheless, it does produce the expected qualitative behavior. For example, it enables a stagnant three-phase mixture to separate into gas, oil and water zones through counter-current flow.

To simulate both steady-state and transient multiphase wellbore flows, and to enable the optimization of transient drift-flux parameters, a transient wellbore flow model, MSWell has been developed. This model will be enhanced and implemented into GPRS to allow the simulation of multiphase flow in wellbores and possibly surface facilities.

This chapter proceeds as follows. We first present the detailed drift-flux model used in this work. We then provide an overview of the experimental program and describe the type and quality of the data collected. Next, we compute drift-flux model parameters from this data using an optimization technique that minimizes the difference between the experimental 
data and model predictions for steady state water-gas, oil-water and oil-water-gas flows. The effect of pipe inclination is also modeled. Then, the development of MSWell is described. We note that the material presented in sections 5.2 - 5.6 appeared in two SPE papers (Shi et al., 2003, 2004).

\subsection{Drift-flux Model}

The drift-flux model for two-phase flow (Zuber and Findlay, 1965) describes the slip between gas and liquid as a combination of two mechanisms. One mechanism results from the non-uniform profiles of velocity and phase distribution over the pipe cross-section (see Fig. 5-1). The gas concentration in vertical gas-liquid flow tends to be highest in the center of the pipe, where the local mixture velocity is also fastest. Thus, when integrated across the area of the pipe, the average velocity of the gas tends to be greater than that of the liquid. The other mechanism results from the tendency of gas to rise vertically through the liquid due to buoyancy.

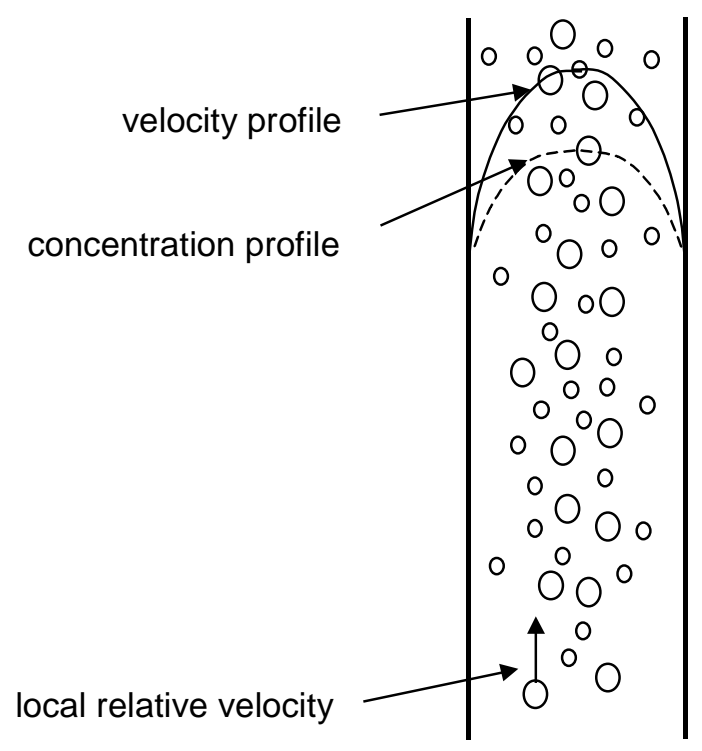

Figure 5-1: Profile and local slip mechanisms in the drift-flux model

A formulation that combines the two mechanisms is (Zuber and Findlay, 1965):

$$
V_{g}=C_{0} V_{m}+V_{d}
$$


Here $V_{g}$ is the flow velocity of the gas phase, averaged across the pipe area, $C_{0}$ is the profile parameter (or distribution coefficient), which describes the effect of the velocity and concentration profiles, $V_{m}$ is the volumetric flux (or average velocity) of the mixture and $V_{d}$ is the drift velocity of the gas, describing the buoyancy effect.

The average mixture velocity is the sum of the gas and liquid superficial velocities,

$$
V_{m}=V_{s g}+V_{s l}=\alpha_{g} V_{g}+\left(1-\alpha_{g}\right) V_{l}
$$

where $\alpha_{g}$ is the in situ gas volume fraction, averaged across the area of the pipe. The average flow velocity of the liquid phase is thus

$$
V_{l}=\frac{1-\alpha_{g} C_{0}}{1-\alpha_{g}} V_{m}-\frac{\alpha_{g}}{1-\alpha_{g}} V_{d}
$$

For efficient application in a well model of a reservoir simulator, we require expressions for $C_{0}$ and $V_{d}$ that are relatively simple to compute, continuous and differentiable. As will be evident below, some of the characteristics of the different flow patterns can be captured through these parameters.

\subsubsection{Profile Parameter}

Zuber and Findlay (1965) reported values of $C_{0}$ ranging between 1.0 and 1.5. Several driftflux models use a value for $C_{0}$ of 1.2 in the bubble and slug flow regimes (e.g., Aziz et al., 1972; Ansari et al., 1994; Hasan and Kabir, 1988a), but in the annular mist regime the value is close to 1.0. Moreover, $C_{0}$ should approach 1.0 as $\alpha_{g}$ approaches 1.0; in fact $\alpha_{g} C_{0}$ should never exceed 1.0. Accordingly, we apply a relationship for $C_{0}$ that has a constant value at conditions equivalent to bubble or slug flow, and reduces to 1.0 as $\alpha_{g}$ approaches 1.0 or as

the mixture velocity increases. A suitable expression with these properties is (Schlumberger GeoQuest, 2001)

$$
C_{0}=\frac{A}{1+(A-1) \gamma^{2}}
$$

The purpose of the term involving $\gamma$ is to cause $C_{0}$ to reduce to 1.0 at high values of $\alpha_{g}$ or $V_{m}$. The $\gamma$ parameter is given by 


$$
\gamma=\frac{\beta-B}{1-B} \quad \text { subject to the limits } \quad 0 \leq \gamma \leq 1
$$

where $\beta$ is a quantity that approaches 1.0 as $\alpha_{g}$ approaches 1.0 , and also as the mixture velocity approaches a high value. We choose the velocity of the onset of the annular flow regime to be the velocity at which the profile slip vanishes. The transition to annular flow occurs when the gas superficial velocity $V_{s g}$ reaches the 'flooding' value $V_{s g f}$ that is sufficient to prevent the liquid film from falling back against the gas flow. An expression for the flooding velocity is given below in Eq. 5-10. Accordingly, we choose the following expression for $\beta$ (Schlumberger GeoQuest, 2001)

$$
\beta=\max \left(\alpha_{g}, F_{v} \frac{\alpha_{g}\left|V_{m}\right|}{V_{s g f}}\right)
$$

The parameters $A, B$ and $F_{v}$ can be tuned to fit the observations. $A$ represents the value of the profile parameter in the bubble and slug flow regimes, and is originally set to be 1.2 in ECLIPSE. $B$ represents the value of the gas volume fraction, or the mixture velocity as a fraction of the flooding velocity, at which $C_{0}$ starts to drop below $A$. $B$ is originally set to be 0.3. $F_{v}$ is a multiplier on the flooding velocity fraction, originally set to be 1.0. Profile flattening can be made more or less sensitive to the velocity by adjusting the value of $F_{v}$.

Intuitively we would expect the gas superficial velocity $V_{s g}=\alpha_{g} V_{g}$ to increase with both $\alpha_{g}$ and $V_{m}$, so the relationship for $C_{0}$ must also satisfy (Schlumberger GeoQuest, 2001)

$$
\frac{\partial}{\partial \alpha_{g}}\left(\alpha_{g} C_{0}\right)>0 \text { and } \frac{\partial}{\partial V_{m}}\left(V_{m} C_{0}\right)>0
$$

There may be problems with stability if this is not the case. Both of these criteria will be satisfied if $B<(2-A) / A$.

\subsubsection{Gas-Liquid Drift Velocity}

We can derive an expression for the gas-liquid drift velocity by combining data on the limits of counter-current flow made under a variety of flow conditions, and interpolating between them to avoid discontinuities. The method honors observations of gas-liquid relative velocities at low and high gas volume fractions, and joins them with a 'flooding curve' 
(Holmes, 1977), as shown in Fig. 5-2. The relative velocity observations relate to the rise velocity of gas through a stationary liquid. From Eqs. 5-1 and 5-3 we can relate this to the drift velocity,

$$
V_{g}\left(V_{l}=0\right)=\frac{V_{d}}{1-\alpha_{g} C_{0}}
$$

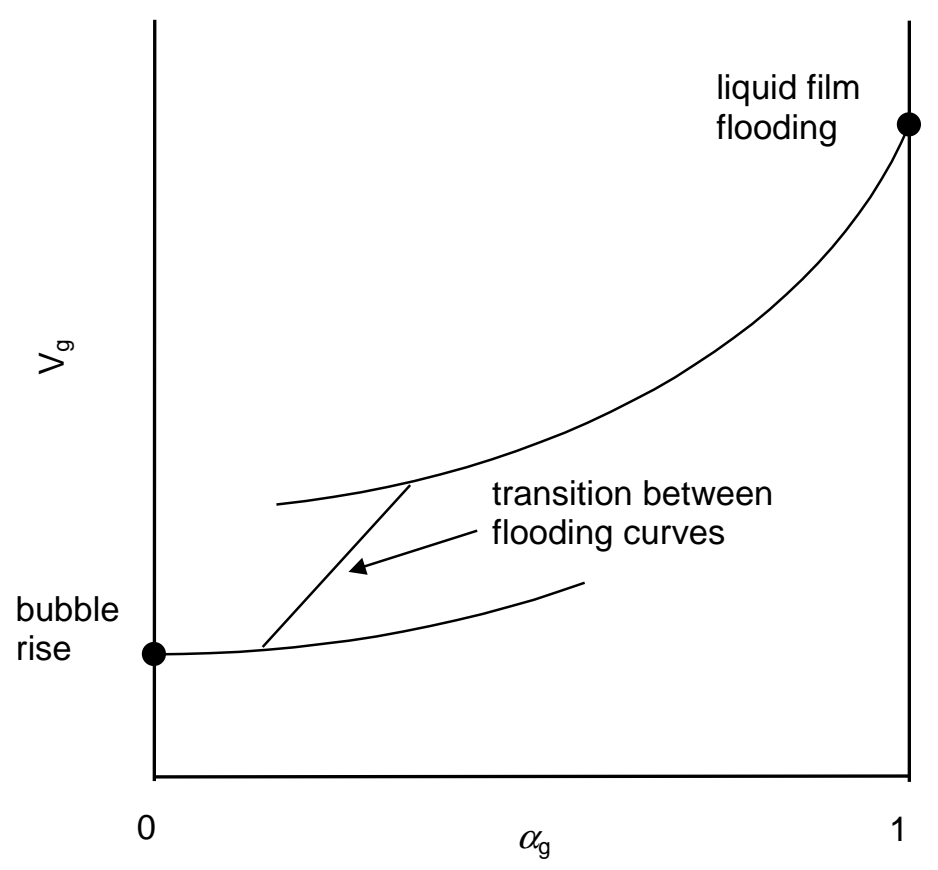

Figure 5-2: Gas rise velocity in a stagnant liquid in the drift-flux model

At low values of $\alpha_{g}$ we use the rise velocity of a bubble through a stagnant liquid, which Harmathy (1960) observed to be $1.53 V_{c}$, where $V_{c}$ is the characteristic velocity given by

$$
V_{c}=\left(\frac{\sigma_{g l} g\left(\rho_{l}-\rho_{g}\right)}{\rho_{l}^{2}}\right)^{1 / 4}
$$

where $\sigma_{g l}$ is the gas-liquid interfacial tension.

At high values of $\alpha_{g}$ we use the 'flooding velocity', defined as the gas velocity that is just sufficient to support a thin annular film of liquid and prevent it from falling back against the gas flow. Wallis and Makkenchery (1974) obtained the relation 


$$
V_{g}\left(V_{l}=0\right)=K_{u}\left(\frac{\rho_{l}}{\rho_{g}}\right)^{1 / 2} V_{c}
$$

where $K_{u}$ is the 'critical Kutateladze number', which is related to the dimensionless pipe diameter

$$
\hat{D}=\left(\frac{g\left(\rho_{l}-\rho_{g}\right)}{\sigma_{g l}}\right)^{1 / 2} D
$$

according to Table 5-1 (Schlumberger GeoQuest, 2001).

Table 5-1. The critical Kutateladze number vs. the dimensionless pipe diameter

\begin{tabular}{|l|l|}
\hline$\hat{D}$ & $K_{u}$ \\
\hline$\leq 2$ & 0 \\
\hline 4 & 1.0 \\
\hline 10 & 2.1 \\
\hline 14 & 2.5 \\
\hline 20 & 2.8 \\
\hline 28 & 3.0 \\
\hline$\geq 50$ & 3.2 \\
\hline
\end{tabular}

To interpolate between these two extremes we make use of the flooding curve described by Wallis (1974) to define the limit of the counter-current flow regime. Wallis observed that the gas and liquid flow rates that mark the limit of steady counter-current flow lie on the curve (Schlumberger GeoQuest, 2001)

$$
\left(\frac{\rho_{g}}{\rho_{l}}\right)^{1 / 4} \sqrt{V_{s g}}+\sqrt{-V_{s l}}=c\left[\frac{\left(\rho_{l}-\rho_{g}\right)}{\rho_{l}} g D\right]^{1 / 4}
$$

where $c$ is a constant that depends on the pipe geometry. The sign convention is that upward flow is positive. We can normalize this curve to meet the flooding velocity observations in Eq. $5-10$ as $\alpha_{g} \rightarrow 1.0$ by substituting the right hand side of Eq. 5-12 with $\sqrt{K_{u} V_{c}}$. Next, note that Eq. 5-1 can be written as

$$
\left(1-\alpha_{g} C_{0}\right) V_{s g}-\alpha_{g} C_{0} V_{s l}=\alpha_{g} V_{d}
$$


Assuming that $C_{0}$ does not vary with the flow velocity in the region of interest (the counter-current flow region), for a given value of $\alpha_{g}$ Eq. 5-13 describes a straight line on a graph with axes $V_{s g}$ and $V_{s l}$. Each of these lines should be tangential to the flooding curve Eq. 5-12 renormalized - which represents the limit of counter-current flow in the quadrant where $V_{s g}>0$ and $V_{s l}<0$. This requirement defines the drift velocity $V_{d}$ as a function of $\alpha_{g}$

$$
V_{d}=\frac{\left(1-\alpha_{g} C_{0}\right) C_{0} K_{u} V_{c}}{\alpha_{g} C_{o} \sqrt{\frac{\rho_{g}}{\rho_{l}}}+1-\alpha_{g} C_{0}}
$$

The curve must be 'ramped' down in order to match Harmathy's bubble rise velocity at low values of $\alpha_{g}$. We apply a linear ramp between two selected values of the gas volume fraction $a_{1}$ and $a_{2}$. The overall relation for the drift velocity is thus (Schlumberger GeoQuest, 2001)

$$
V_{d}=\frac{\left(1-\alpha_{g} C_{0}\right) C_{0} K\left(\alpha_{g}\right) V_{c}}{\alpha_{g} C_{o} \sqrt{\frac{\rho_{g}}{\rho_{l}}}+1-\alpha_{g} C_{0}}
$$

where

$$
\begin{array}{ll}
K\left(\alpha_{g}\right)=1.53 / C_{0} & \text { when } \alpha_{g} \leq a_{1} \\
K\left(\alpha_{g}\right)=K_{u}(\hat{D}) & \text { when } \alpha_{g} \geq a_{2}
\end{array}
$$

and a linear interpolation between these values when $a_{1}<\alpha_{g}<a_{2}$. Setting the values of $a_{1}$ and $a_{2}$ to 0.2 and 0.4 respectively matches the data used by Zuber and Findlay (1965) to demonstrate the transition from the bubble flow regime.

\subsubsection{Oil-Water Slip}

The model described above was developed for gas-liquid flow in vertical pipes. Hasan and Kabir (1999) proposed a drift-flux model for oil-water flow. The slip between oil and water is similarly described as a combination of profile and buoyancy effects

$$
V_{o}=C_{0}^{\prime} V_{l}+V_{d}^{\prime}
$$


For the oil-water profile parameter $C_{0}^{\prime}$ they suggest a value of 1.2, but this should decrease to 1.0 when oil becomes the continuous phase $\left(\alpha_{0}>0.7\right)$. We make $C_{0}^{\prime}$ a continuous function of the oil volume fraction (Schlumberger GeoQuest, 2001),

$$
\begin{aligned}
& C_{0}^{\prime}=A^{\prime} \quad \text { when } \quad \alpha_{o} \leq B_{1}^{\prime} \\
& C_{0}^{\prime}=1.0 \quad \text { when } \quad \alpha_{o} \geq B_{2}^{\prime} \\
& C_{0}^{\prime}=A^{\prime}-\left(A^{\prime}-1\right)\left(\frac{\alpha_{o}-B_{1}^{\prime}}{B_{2}^{\prime}-B_{1}^{\prime}}\right) \quad \text { when } \quad B_{1}^{\prime}<\alpha_{o}<B_{2}^{\prime}
\end{aligned}
$$

The parameters $A^{\prime}, B_{1}^{\prime}$ and $B_{2}^{\prime}$ are adjustable and are originally set to $1.2,0.4$ and 0.7 respectively. A condition equivalent to Eq. 5-7 - that the oil superficial velocity should increase with the oil volume fraction - requires that $B_{1}^{\prime}<\left(2-A^{\prime}\right) B_{2}^{\prime}$.

For the oil-in-liquid drift velocity Hasan and Kabir (1999) suggest

$$
V_{d}^{\prime}=1.53 V_{c}^{\prime}\left(1-\alpha_{o}\right)^{2}
$$

where $V_{c}^{\prime}$ is the characteristic velocity (Eq. 5-9) derived with oil and water properties,

$$
V_{c}^{\prime}=\left(\frac{\sigma_{o w} g\left(\rho_{w}-\rho_{o}\right)}{\rho_{w}^{2}}\right)^{1 / 4}
$$

For oil-dominated flow $\left(\alpha_{o}>0.7\right)$ they recommend switching to a no-slip model. But, because Eq. 5-18 has already reduced the drift velocity by an order of magnitude when the oil volume fraction reaches 0.7 , we retain that relation over the full range of $\alpha_{o}$ in order to maintain continuity.

\subsubsection{Three-Phase Flow}

To model three-phase (oil, water and gas) flow, we take a two-stage approach that uses the available two-phase flow models. First we combine the oil and water into a single liquid phase, with flow-weighted average properties, and determine the flow velocities of the gas and liquid phases using Eq. 5-1. We then determine the oil and water velocities within the liquid phase using Eq. 5-16. For this calculation we use the oil volume fraction in the liquid phase, $\alpha_{o l}=\alpha_{o} /\left(\alpha_{o}+\alpha_{w}\right)$. This very simplistic approach ignores any effect that the 
presence of a third phase may have on the respective two-phase flow models, and so must be regarded as highly tentative. Nevertheless, it does produce the expected qualitative behavior, enabling a stagnant three-phase mixture to separate into gas, oil and water zones through counter-current flow.

\subsubsection{Inclined Flow}

The relationships described above are based on observations for vertical flow. Hasan and Kabir (1999) proposed the following scaling for the terminal rise velocity for oil-water flow in inclined pipes

$$
V_{\infty \theta}=V_{\infty}(\cos \theta)^{0.5}(1+\sin \theta)^{2}
$$

where $\theta$ is the angle of deviation from the vertical. The terminal rise velocity of a bubble or droplet is equivalent to $V_{d}$ as $\alpha_{o}$ approaches zero. The rise velocity increases to about 2.5 times its value in vertical pipes, before falling off steeply to zero when the pipe is horizontal. Hasan and Kabir (1999) presented their scaling as valid for oil-water flow for deviations $\theta<$ $70^{\circ}$. The application of Eq. 5-20 outside these conditions and over the complete range of $\alpha$ can only be viewed as a tentative procedure.

\subsection{Experimental Setup and Results}

We now briefly describe the experimental setup and data that will be used for our determination of the drift-flux parameters. For a full description, see Oddie et al. (2003).

\subsubsection{Experimental Setup}

The experiments were conducted in a $10.9 \mathrm{~m}$ long, $15.2 \mathrm{~cm}$ diameter, inclinable flow-loop. The test section, shown in Fig. 5-3, is made of plexiglass to enable visual observations. During the experiments, the pipe deviation varied from $0^{\circ}$ (upwards vertical) to $92^{\circ}$ (slightly downhill). Oil (kerosene with a viscosity of $1.5 \mathrm{cP}$ and a density of $810 \mathrm{~kg} / \mathrm{m}^{3}$ at $18^{\circ} \mathrm{C}$ ) and water (tap water) were stored in a large separator and were transported separately to the inlet chamber. Another tank containing liquid nitrogen supplied gas to the pipe. This gas was first passed through evaporators and heat exchangers to allow it to reach ambient temperature. 
Oil, water and gas entered the pipe and flowed along the test section, where the flow pattern was observed and the pressure and holdup (as described below) were measured.

Once steady state was achieved and the steady state measurements were completed, the test section was shut in using fast closing valves. After the fluids settled, the flow-loop was rotated to the vertical if necessary so that the final positions of the fluid interfaces could be measured directly from markings on the test section. This provided the shut-in holdup. Over most of the range in holdup the error of this absolute volume measurement is less than $1 \%$. However, volume fractions greater than $94 \%$ could not be measured accurately since the part of the test section near the outlet is manufactured from steel and so is not transparent.

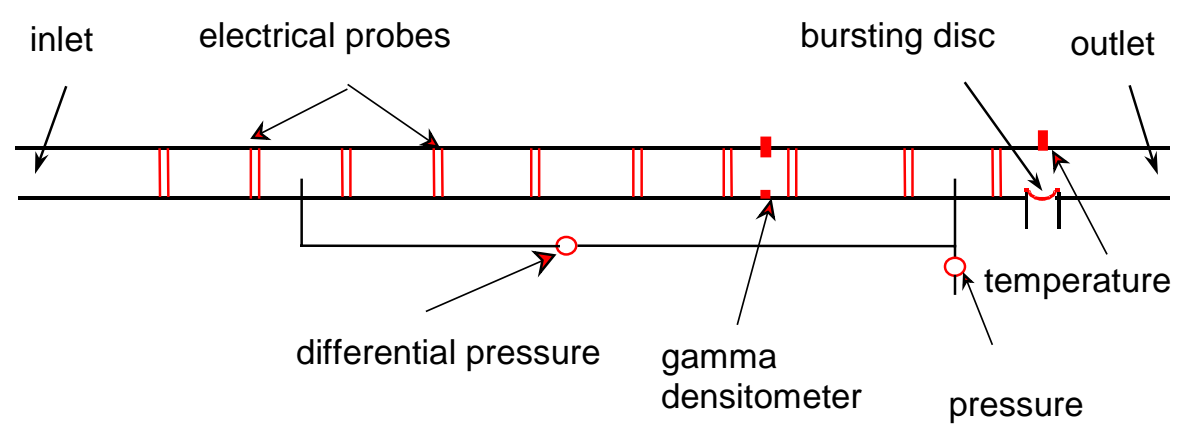

Figure 5-3: Schematic of the test section of the flow loop

The other two methods used to estimate the steady state holdup were nuclear and electric probe measurements. The nuclear gamma densitometer, instrumented $7.5 \mathrm{~m}$ from the inlet (see Fig. 5-3), measures the mean density of the fluid, from which the steady state holdup can be calculated. Ten electric probes were installed at various axial positions along the pipe. These probes detect the local fluid conductance, which can be used to determine the water holdup. The probes provide estimates for water holdup for both steady state and transient flows (the period following shut-in constitutes the transient stage).

The experiments were carried out over a wide range of flow rates for water, oil and gas (designated $Q_{w}, Q_{o}, Q_{g}$ ). For water-gas flow, $2 \leq Q_{w} \leq 100 \mathrm{~m}^{3} / \mathrm{h}$ and $5 \leq Q_{g} \leq 100 \mathrm{~m}^{3} / \mathrm{h}$ was examined, while for oil-water flow $2 \leq Q_{o} \leq 40 \mathrm{~m}^{3} / \mathrm{h}$ and $2 \leq Q_{w} \leq 130 \mathrm{~m}^{3} / \mathrm{h}$ was investigated. For three phase flows, the flow rate ranges were $2 \leq Q_{o} \leq 40 \mathrm{~m}^{3} / \mathrm{h}, 5 \leq Q_{w} \leq 40 \mathrm{~m}^{3} / \mathrm{h}$, and $5 \leq$ 
$Q_{g} \leq 50 \mathrm{~m}^{3} / \mathrm{h}$. Different combinations of flow rates were used for the different types of tests. Note that $1 \mathrm{~m}^{3} / \mathrm{h} \approx 151 \mathrm{bbl} / \mathrm{day}$; thus the maximum rate experiments correspond to flow rates of about 20,000 bbl/day. Each combination of flow rates was repeated at eight pipe deviations $\left(0^{\circ}, 5^{\circ}, 45^{\circ}, 70^{\circ}, 80^{\circ}, 88^{\circ}, 90^{\circ}\right.$, and $92^{\circ}$ from upward vertical $)$.

In this study, two-phase and three-phase steady state data for water-gas, oil-water and oilwater-gas flows are used for the determination of drift-flux parameters. We therefore present sample data for these three types of flows.

\subsubsection{Steady State Holdup Results}

As holdup is one of the most important quantities characterizing multiphase flow in wellbores, three techniques (as discussed in the previous section) were used to assess steady state holdup. We compared the three measurements in detail (see Oddie et al., 2003) and concluded that the resulting holdups were quite consistent overall. Since the shut-in measurement is a direct measurement, with a measurement error of less than $1 \%$ for holdup values less than $94 \%$, we used it to represent steady state holdup. We now present a few experimental results for shut-in water holdup (referred to simply as "holdup") to provide an indication of the type and range of experimental data that will be used to determine the driftflux model parameters.

Fig. 5-4 displays experimental results for water-gas systems. We present results at vertical and $45^{\circ}$ deviation for three gas flow rates. This and subsequent plots show data in terms of water holdup versus the input water fraction or water cut $\left(C_{w}\right)$. For a water-gas system, $C_{w}=Q_{w} /\left(Q_{w}+Q_{g}\right)$. The error bars indicated in the figures are $\pm 5 \%$, as determined from repeated experiments. The vertical distance from any point to the $\alpha_{w}=C_{w}$ line gives the slip between the two phases. As gas flow rate increases, slip decreases for constant input fractions. The slip also decreases as $C_{w}$ increases and as the pipe deviation shifts from $45^{\circ}$ to vertical. As shown in Fig. 5-5, when the pipe is further deviated from $45^{\circ}$ to $80^{\circ}$, the slip decreases. This, taken along with our measurements at $70^{\circ}$ deviation (Oddie et al., 2003), indicates that the slip displays a maximum between $45^{\circ}$ and $70^{\circ}$. As we will see, the driftflux parameters determined from the data capture this effect. 


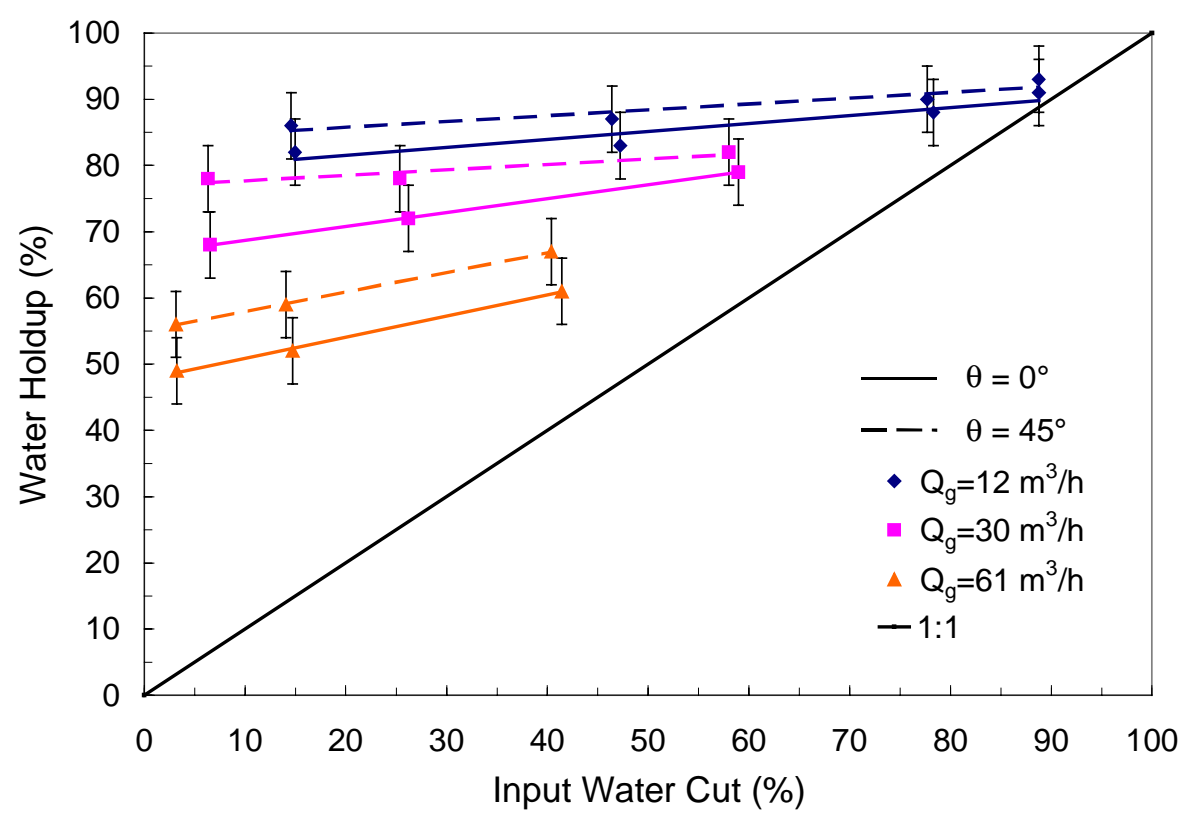

Figure 5-4: Holdup for water-gas system for $\theta=0^{\circ}$ and $\theta=45^{\circ}$

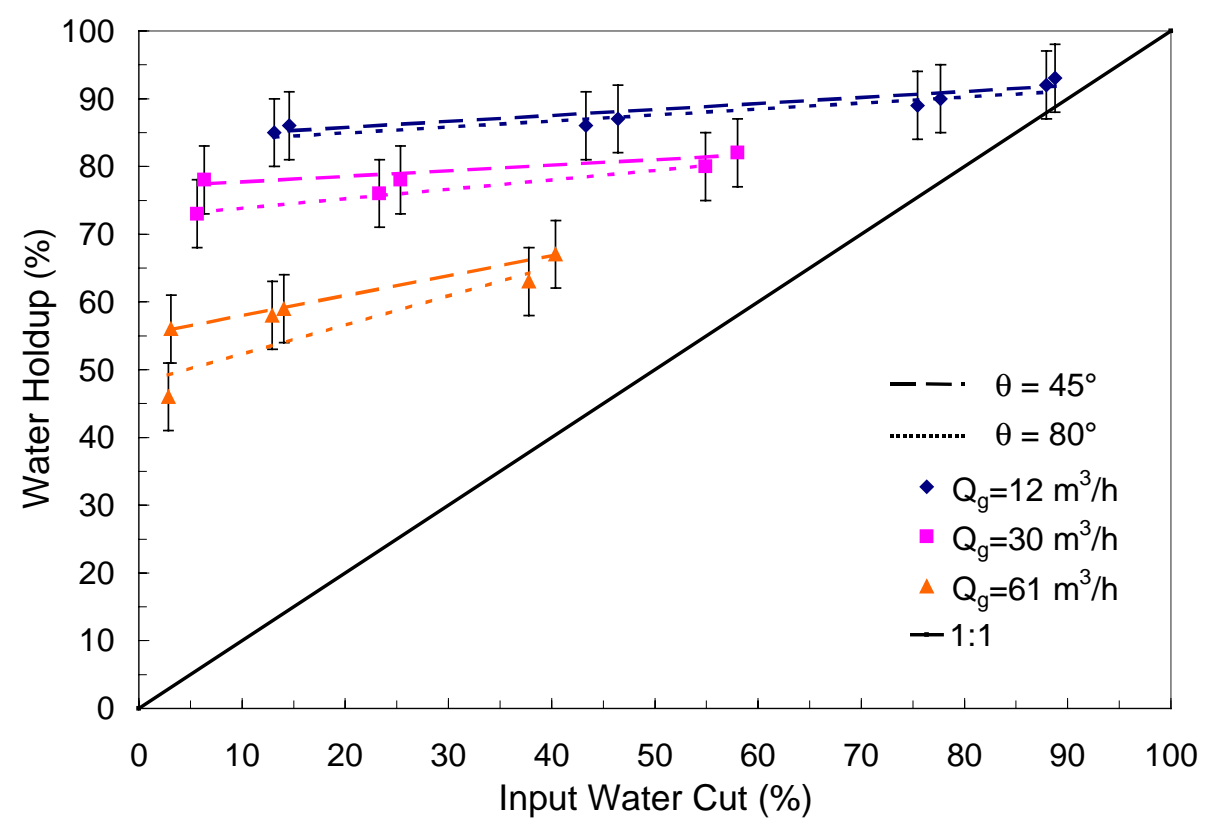

Figure 5-5: Holdup for water-gas system for $\theta=45^{\circ}$ and $\theta=80^{\circ}$ 
Experimental results for oil-water systems, for vertical flow and flow at $70^{\circ}$ deviation, are presented in Fig. 5-6. Qualitatively similar trends can be observed here as in Figs. 5-4 and 5-5, though the magnitude of the effects is quite different. For example, at high flow rates $\left(Q_{o}=40 \mathrm{~m}^{3} / \mathrm{h}\right)$ there is very little slip at either inclination. At intermediate flow rates $\left(Q_{o}=\right.$ $10 \mathrm{~m}^{3} / \mathrm{h}$ ), by contrast, the effect of inclination is very pronounced, with much higher slip observed at $70^{\circ}$. Again, our intent is to capture these complex effects via the optimized driftflux parameters.

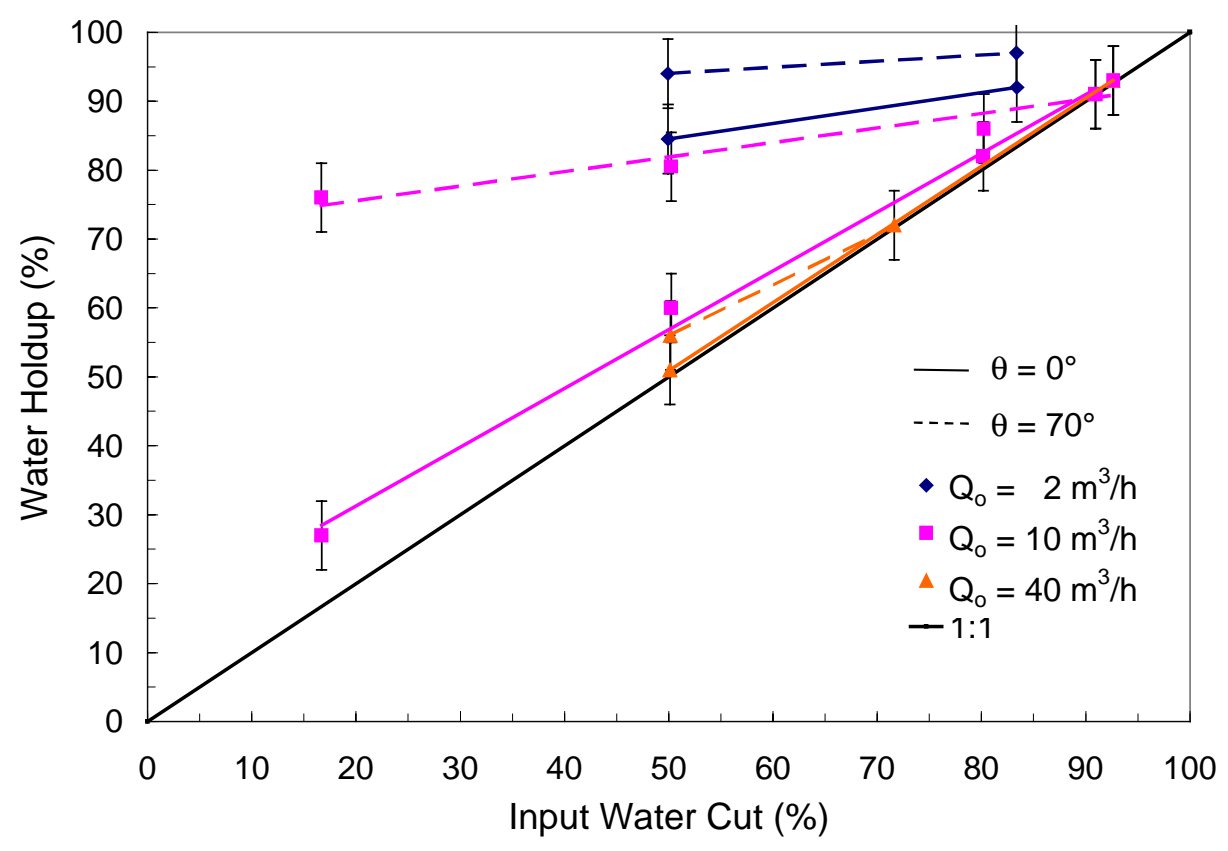

Figure 5-6: Holdup for oil-water system for $\theta=0^{\circ}$ and $\theta=70^{\circ}$

Fig. 5-7 shows a sample of the three-phase experimental data. Here we combine water and oil into a single liquid phase and plot the liquid holdup $\alpha_{l}\left(\alpha_{l}=\alpha_{o}+\alpha_{w}\right)$ versus input liquid volume fraction $\left(C_{l}\right)$ to show the slip between liquid and gas. The slip between oil and water will be considered in detail below. For an oil-water-gas system, $C_{l}=\left(Q_{w}+Q_{o}\right) /\left(Q_{w}+Q_{o}+Q_{g}\right)$. The diagonal line in Fig. 5-7 is the no-slip line, which represents a homogeneous liquid-gas flow; i.e., $\alpha_{l}=C_{l}$. The data are for vertical and $45^{\circ}$ deviation for different gas flow rates. 


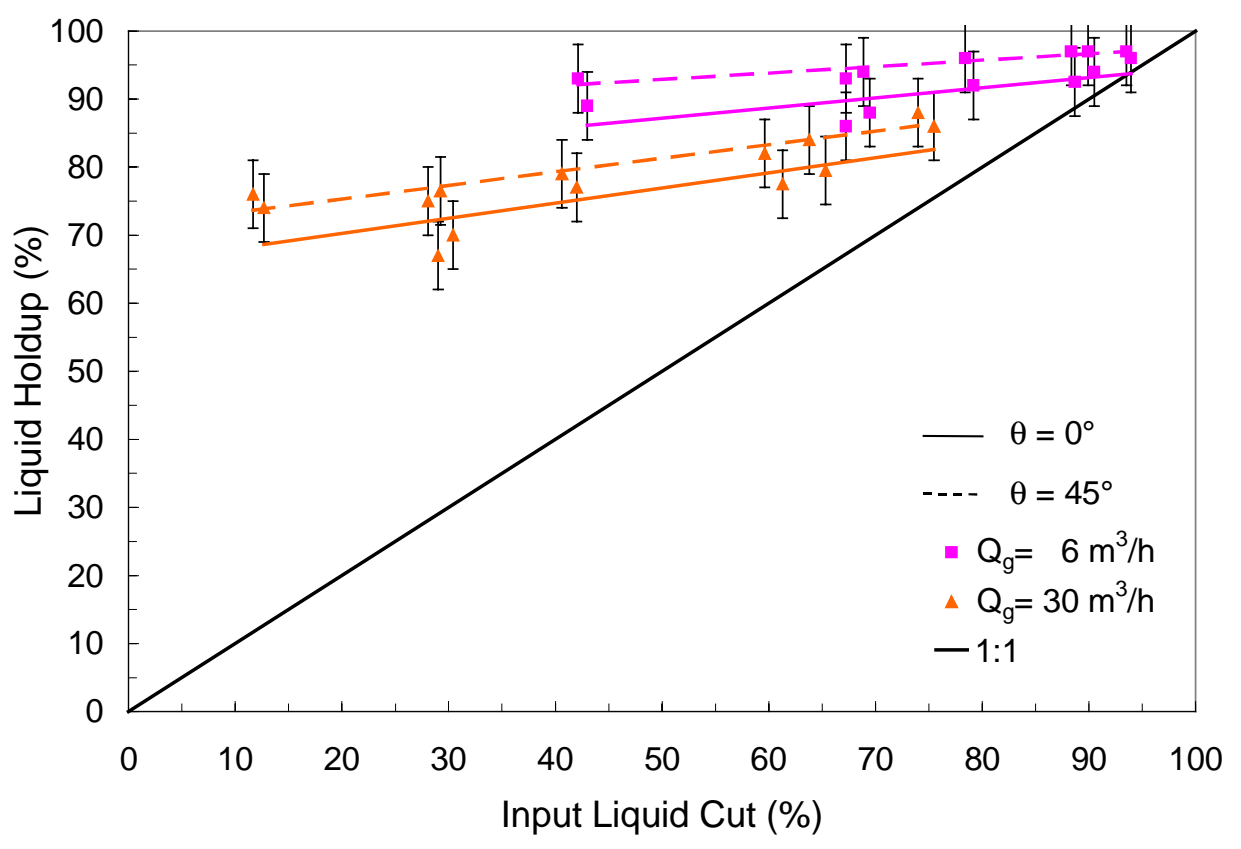

Figure 5-7: Holdup for oil-water-gas system for $\theta=0^{\circ}$ and $\theta=45^{\circ}$.

It is apparent from the figure that the slip between liquid and gas is quite significant. For example, for an input liquid cut of about 10\%, the in situ liquid percentage can be as high as $70 \%$. The effect of deviation, gas flow rate, and $C_{l}$ are clearly displayed in the figure. As the pipe is deviated from vertical to $45^{\circ}$, the slip increases. With increasing gas flow rate, the slip decreases for a constant input fraction. The slip also decreases as $C_{l}$ increases. Comparing the data shown here with data at $70^{\circ}$ deviation (Oddie et al., 2003), we see that there is a similar level of slip at both $45^{\circ}$ and $70^{\circ}$ inclinations. Trends similar to those shown in Fig. 57 are also observed in Figs. 5-4 and 5-5, which suggests that the slip between liquid and gas in three-phase flow may be similar to that in water-gas flow.

\subsection{Parameter Determination}

In this section we describe the optimization procedure used to determine the drift-flux parameters. The parameters are determined such that the difference between the experimental data and model predictions for holdup are minimized in a least-square sense. We first discuss water-gas and oil-water systems and then consider oil-water-gas flows. For all three systems we use experimental data for only the 6 deviations from $0^{\circ}$ to $88^{\circ}$ (inclusive) for the 
parameter determination. This is because the experimental holdup data for $90^{\circ}$ and $92^{\circ}$ display relatively large errors due to the way in which fluid exited the test section (Oddie $e t$ al., 2003).

From our earlier description of the drift-flux model, it is apparent that there are many parameters that could potentially be modified to generate close fits between the experimental and model predictions. However, we wish to avoid overly tuning the model as this may result in some parameters assuming unphysical values or in an unnecessarily complex description. We therefore performed a number of optimizations holding different sets of parameters fixed (and varying others) to determine an appropriate balance between model complexity and closeness of fit.

The parameters we ultimately chose to optimize were selected primarily based on two considerations. We optimized parameters to which the model is most sensitive as well as parameters that are the most uncertain. For example, for the water-gas system, seven parameters were optimized. These include $A$ and $B$, which define the shape of the $C_{o}$ versus $\alpha_{g}$ curve (see Fig. 5-8), as well as $a_{1}$ and $a_{2}$, which define the transitions in the $V_{d}$ versus $\alpha_{g}$ curve (Fig. 5-9). The remaining parameters optimized define the drift velocity multiplier $m$. This multiplier is especially useful in matching data from inclined pipes, where the original relationship is given by Eq. (5-20). Specifically, from Eq. (5-20), the default representation for $V_{\infty \theta}$ can be written as

$$
V_{\infty \theta}=V_{\infty} m(\theta)
$$

where $m(\theta)$ gives the inclination effect (Hasan and Kabir, 1999)

$$
m(\theta)=(\cos \theta)^{0.5}(1+\sin \theta)^{2}
$$

We parameterize $m(\theta)$ as

$$
m(\theta)=m_{0}(\cos \theta)^{n_{1}}(1+\sin \theta)^{n_{2}}
$$

Here $m_{0}$ is used to account for a non-unit multiplier for vertical flow; $m_{0}, n_{1}$ and $n_{2}$ are all adjustable parameters. 


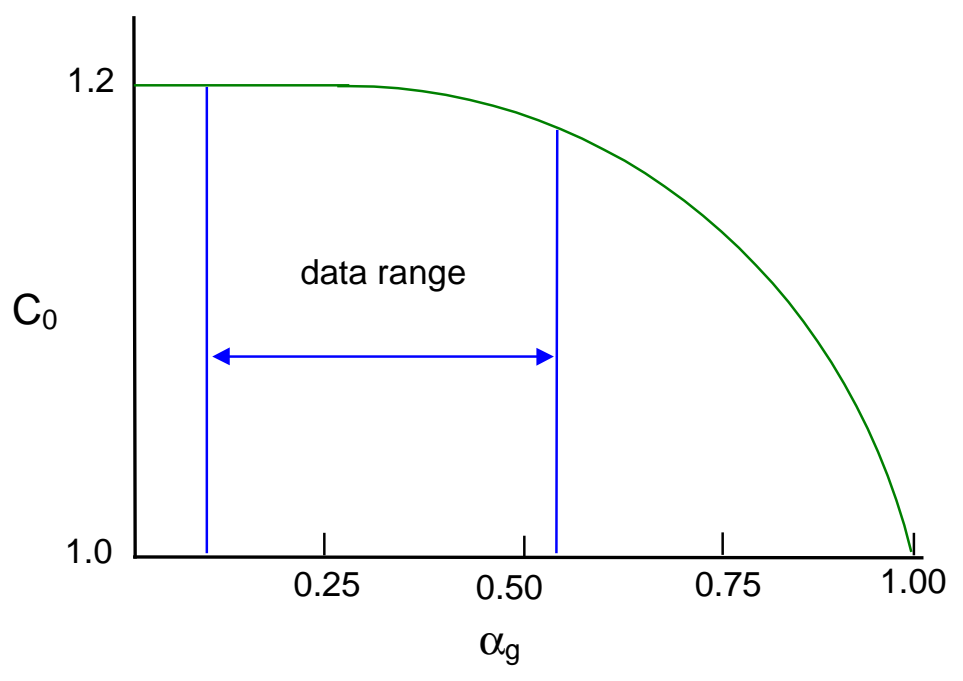

Figure. 5-8: Default profile parameter for water-gas system (Schlumberger GeoQuest, 2001)

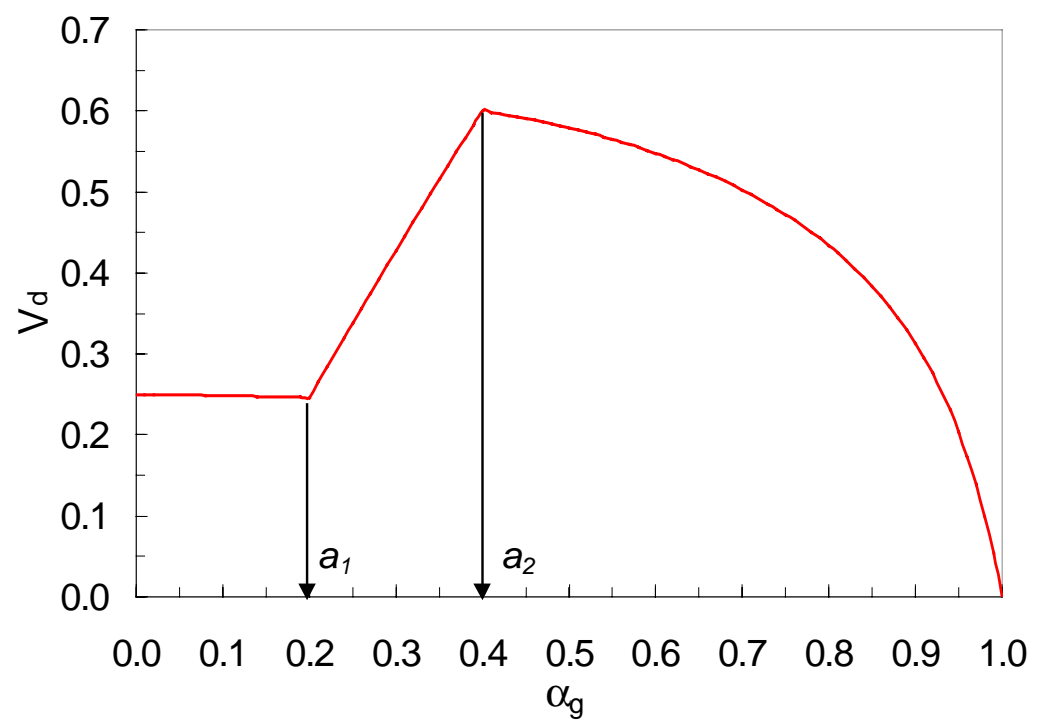

Figure 5-9: Default drift velocity for water-gas system in $15.2 \mathrm{~cm}$ diameter pipe (Schlumberger GeoQuest, 2001)

The model is sensitive to all seven of these parameters. The $a_{1}$ and $a_{2}$ transition parameters are quite uncertain as they were introduced to interpolate between models describing the two limiting cases. 
The vector $X_{P}$ containing the seven parameters to be optimized for the water-gas system is as follows:

$$
X_{P}=\left[\begin{array}{lllllll}
A & B & a_{1} & a_{2} & m_{0} & n_{1} & n_{2}
\end{array}\right]^{T}
$$

The parameters are tuned to minimize the error between the experimental $\alpha_{g}$ and the $\alpha_{g}$ estimated from the drift-flux model (designated $\alpha_{g}^{*}$ ) for water-gas flows. The objective function is

$$
E_{\alpha_{g}}=\sum_{i=1}^{N} w_{i}\left|\alpha_{g_{i}}-\alpha_{g i}^{*}\right|^{2}
$$

where $N$ is the total number of experimental data points and $w_{i}$ is the weight assigned to each point, (i.e., $w_{i}=0.5$ for each of two repeated tests) and the experimental error. For example, experimental $\alpha_{g}$ (or $\alpha_{o}$ ) less than $6 \%$ are assigned a very small value of $w_{i}$ since the exact value could not be measured in this range. The minimization of $E_{\alpha_{g}}$ in Eq. 5-24 is a nonlinear least-square problem. We apply a trust region reflective Newton algorithm in MATLAB (2002) for this minimization.

The calculated gas holdup, computed via

$$
\alpha_{g_{i}}^{*}=\frac{V_{s g_{i}}}{C_{o}\left(\alpha_{g}^{*}, X_{P}\right) V_{m_{i}}+V_{d}\left(\alpha_{g}^{*}, X_{P}\right)}
$$

is obtained from the drift-flux model and the vector of parameters $X_{P}$. Because Eq. 5-25 is implicit in $\alpha_{g_{i}}^{*}$, it must be solved using an iterative procedure. In this work a successive substitution technique is applied. The initial guess for $\alpha_{g_{i}}^{*}$ in Eq. 5-25 is the experimental value $\alpha_{g_{i}}$. The ranges over which the parameters in $X_{P}$ may change during the optimization are specified to limit them to physically allowable values. For example, we require $1.2 \leq A \leq$ 1.5 to maintain consistency with previous findings.

For oil-water systems, we again select seven parameters for optimization. Three of these parameters are analogous to those considered above: $A^{\prime}, B_{1}^{\prime}$ and $B_{2}^{\prime}$ (which define $C_{0}^{\prime}$ ). We 
introduce an additional parameter in the expression for the oil-water drift velocity. This new parameter, designated $n^{\prime}$, replaces the exponent 2 in Eq. 5-18. The drift-flux multiplier $m^{\prime}(\theta)$ is parameterized as

$$
m^{\prime}(\theta)=n_{1}^{\prime} \cos \theta+n_{2}^{\prime} \sin 2 \theta+n_{3}^{\prime} \sin 3 \theta
$$

where $n_{1}^{\prime}, n_{2}^{\prime}$, and $n_{3}^{\prime}$ are adjustable parameters. The vector of parameters to be optimized, $X_{P}^{\prime}$, is:

$$
X_{P}^{\prime}=\left[\begin{array}{lllllll}
A^{\prime} & B_{1}^{\prime} & B_{2}^{\prime} & n^{\prime} & n_{1}^{\prime} & n_{2}^{\prime} & n_{3}^{\prime}
\end{array}\right]^{T}
$$

For both water-gas and oil-water flows, we optimize the parameter values for the combined data from all 6 deviations. The optimization starts with multiple initial guesses (on the order of thousands). Using this unified optimization method with the multiple starting points, we can ensure the best parameter values for the entire data range and parameter space. See Diaz (2004) for further details.

\subsection{Results and Discussion for Two-Phase Flows}

We now present the results for the parameter optimizations for water-gas and oil-water flows.

We present results in terms of calculated $\alpha_{g}$ or $\alpha_{o}$ versus experimental $\alpha_{g}$ of $\alpha_{o}$. Error is quantified as the root mean square average of the absolute error of each point.

\subsubsection{Water-gas Flows}

As discussed in the Introduction, the original parameters currently used in the ECLIPSE simulator were estimated, in many cases, from experiments on small-diameter (e.g., 2 inch) pipes. Thus, we would expect there to be some degree of error when these parameters are applied for large-diameter pipeflow. The predictions for $\alpha_{g}$ for deviations over the range $0 \leq$ $\theta \leq 88^{\circ}$, using these original parameters, are shown in Fig. 5-10. Note that we only include data for $\alpha_{g}>0.06$. Also shown in the figure are dashed lines designating errors of $\pm 10 \%$ and $\pm 20 \%$. There is a clear tendency toward overprediction for $0.1<\alpha_{g}<0.3$. The error is 0.067 .

Using the optimized parameters, we achieve the results shown in Fig. 5-11. The error here is reduced considerably to 0.0222 and all of the data now fall within the $\pm 20 \%$ range. 
This improvement appears to be largely due to the reduction of $a_{1}$ and $a_{2}$ from their default values. It is worth reiterating that only $V_{d}$ depends on the pipe inclination (through $m$ ), and that none of the parameters related to $C_{0}$ varies with $\theta$.

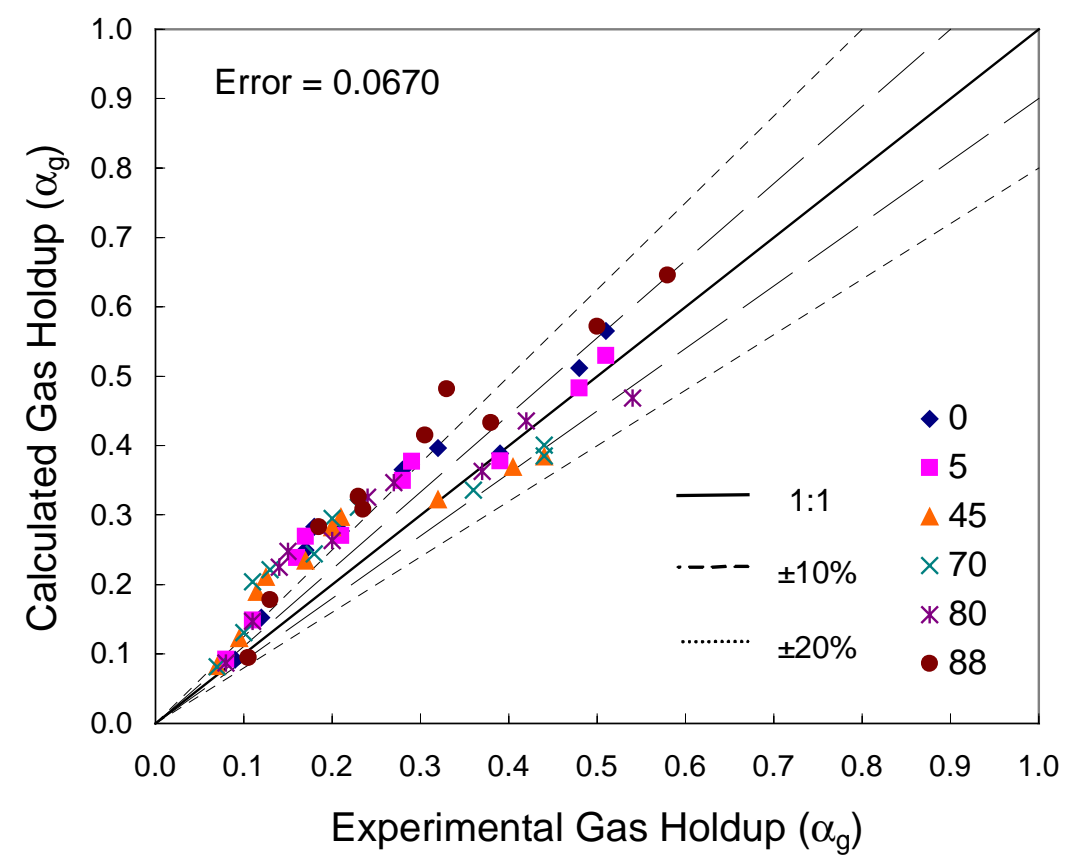

Figure 5-10: Two-phase gas holdup using original ECLIPSE parameters

$\left(A=1.2, B=0.3, a_{1}=0.20, a_{2}=0.40\right.$, Eq. 5-22) 


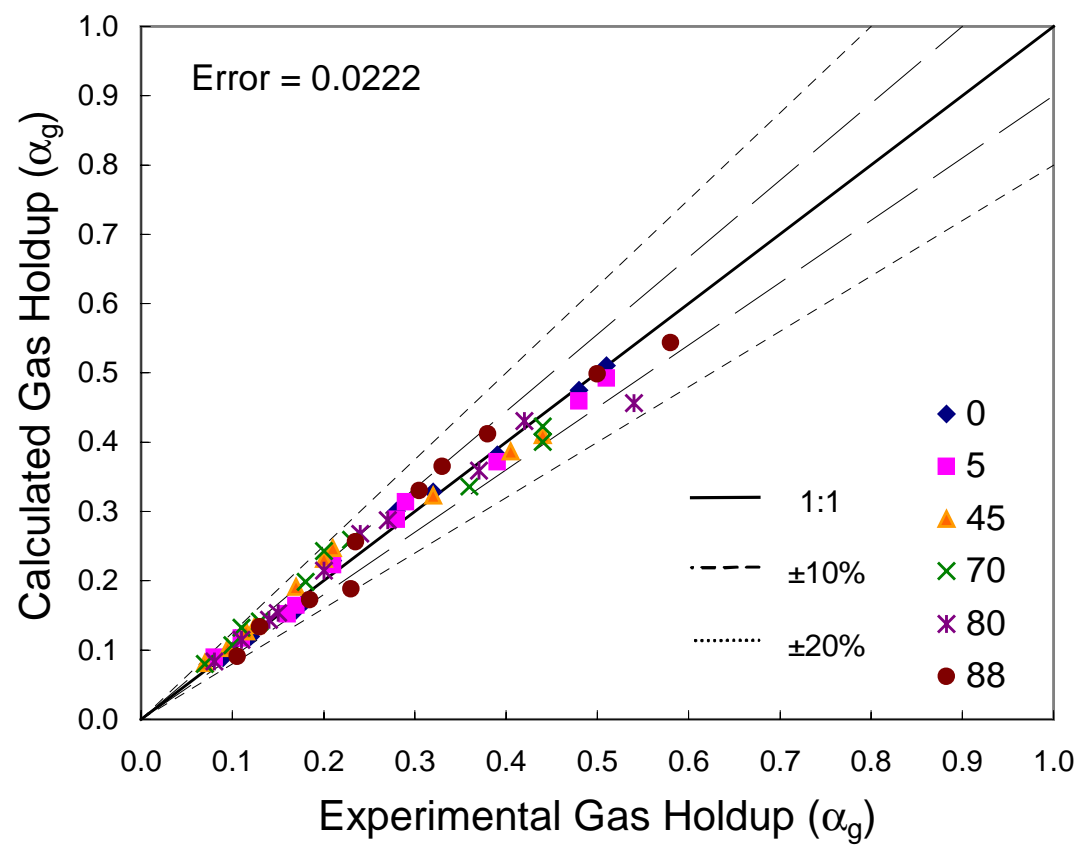

Figure 5-11: Two-phase gas holdup using optimized parameters $\left(A=1.0, a_{1}=0.06, a_{2}=0.21\right.$, Eq. 5-27)

The optimized parameters used for these predictions are:

$$
\begin{aligned}
& A=1.0, a_{1}=0.06, a_{2}=0.21, \text { and } \\
& m(\theta)=1.85(\cos \theta)^{0.21}(1+\sin \theta)^{0.95}
\end{aligned}
$$

Note, in contrast to $A=1.2$, as appropriate for small-diameter systems, the best fit here is $A$ $=1.0$, which means that $C_{0}=1$ for the entire range of $\alpha_{g}$, and the value of $B$ does not affect the model. This indicates that there is no profile effect. However, if we forced $A=1.2$ and $B=0.60$, we obtain

$$
\begin{aligned}
& a_{1}=0.06, a_{2}=0.12, \\
& m(\theta)=1.27(\cos \theta)^{0.24}(1+\sin \theta)^{1.08}
\end{aligned}
$$

The error with these parameters is 0.0264 (compared to 0.0222 in Fig. 5-11). This illustrates that different sets of parameter values can provide fits of similar accuracy. This does not appear to be a major concern, however, as the predicted $\alpha_{g}$ are in close agreement with the experimental data in either case. 
The variation of $m$ with $\theta$ is shown in Fig. 5-12. Eq. 5-27 obtained from the optimization is shown as the solid curve. Note that Eq. 5-27 is valid up to $88^{\circ}$, and does not give $V_{d} \rightarrow 0$ as $\theta \rightarrow 90^{\circ}$, meaning that $V_{d}$ is nonzero for horizontal flow. Similar observations have been made for small-diameter systems (Franca and Lahey, 1992). The $m(\theta)$ suggested by Hasan and Kabir (1999) for oil-water flow (Eq. 5-22) is also shown in the Fig. 5-12 as the dotted curve. As indicated by comparing the two lines, the optimized model and the original model in ECLIPSE, $V_{d}$ is much higher in the optimized model. This is mostly because the value of the corresponding $C_{0}$ is 1.0 for Eq. 5-27. As discussed above, when we force $A=1.2$, the Eq. 5-28 provides a similar fit to Hasan and Kabir (1999) correlation for the $m(\theta)$ shown in Fig. 5-12 as the dashed curve.

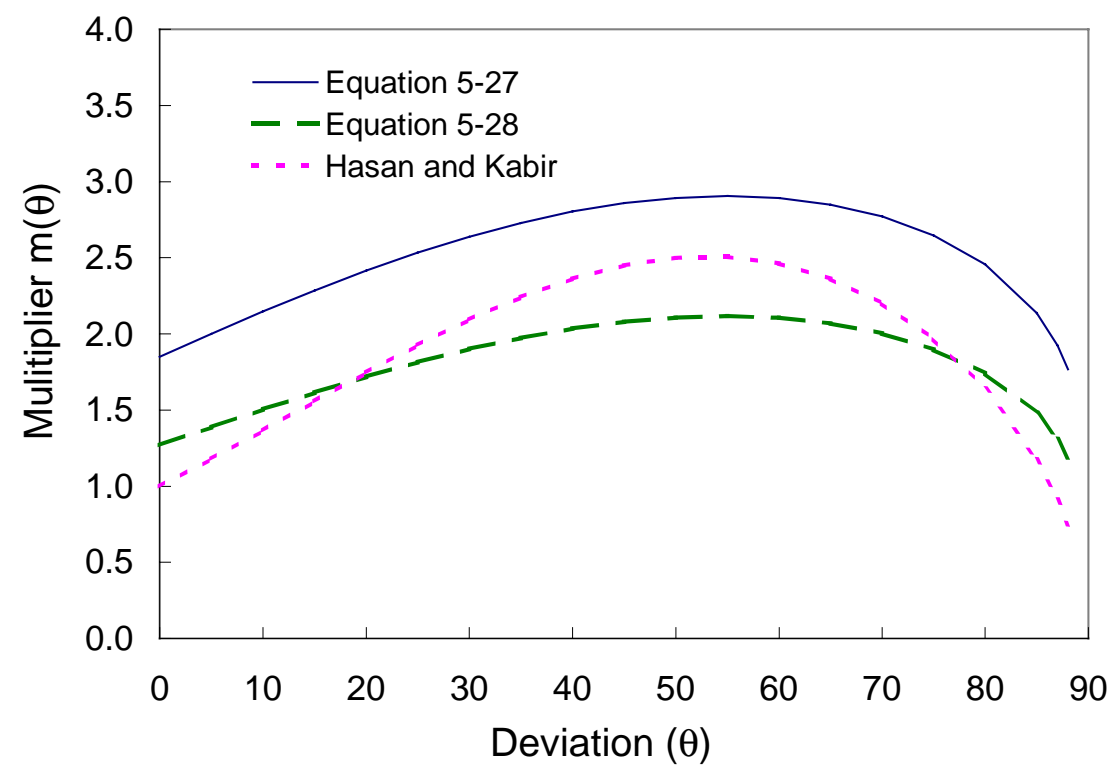

Figure 5-12: Deviation effect for water-gas system

\subsubsection{Oil-Water Flows}

We next consider oil-water systems. The predictions for $\alpha_{o}$ using the original parameter values are shown in Fig. 5-13. The error of 0.135 here is quite significant. A cluster of four points (corresponding to calculated $\alpha_{o}$ of $\sim 0.75$ ) is noticeably outside the $20 \%$ error line. These points all correspond to flows in which $Q_{o}=10 \mathrm{~m}^{3} / \mathrm{h}$ and $Q_{w}=2 \mathrm{~m}^{3} / \mathrm{h}$. These flow rates 
are relatively low, but the input oil cut is the highest investigated. The original parameter values result in significant errors for this combination of flow rates because $V_{d}$ is significantly underestimated, as we will see below.

Predictions using the optimized parameters are shown in Fig. 5-14. Using the optimized set of parameters,

$$
\begin{aligned}
& A^{\prime}=1.0, n^{\prime}=1.0, \text { and } \\
& m^{\prime}(\theta)=1.07 \cos \theta+3.23 \sin 2 \theta-2.32 \sin 3 \theta
\end{aligned}
$$

the average error is reduced to only $23 \%$ of that using the original parameters and the four outliers now cluster around the diagonal. A few points remain outside of the $\pm 20 \%$ range, however. When $A^{\prime}=1.0$, the parameters $B_{1}{ }^{\prime}$ and $B_{2}{ }^{\prime}$ do not enter the model since $C_{0}{ }^{\prime}$ remains constant at 1.0 (see Eq. 5-17). Our finding that $A^{\prime}=1.0$ (which implies that $C_{0}^{\prime}=1.0$ independent of $\left.\alpha_{o}\right)$ is consistent with the earlier result of Hill $(1992,1993)$. He considered oil-water flow in a $16.4 \mathrm{~cm}$ diameter vertical pipe (the oil was kerosene with a density of 780 $\mathrm{kg} / \mathrm{m}^{3}$ and a viscosity of $2 \mathrm{cP}$ ). Using linear regression, Hill found that $C_{0}{ }^{\prime}=1.0$. Our result is in close agreement with this observation.

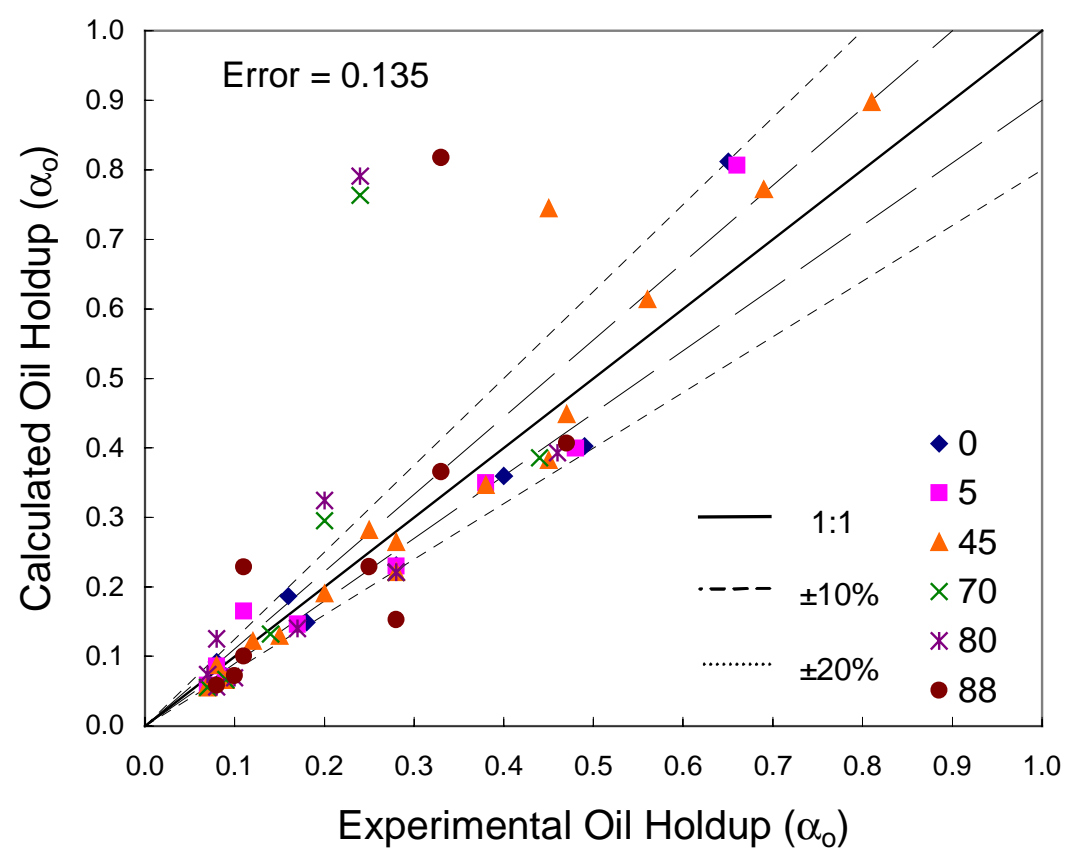

Figure 5-13: Predicted oil holdup using original ECLIPSE parameters $\left(A^{\prime}=1.2, B_{1}=0.4, B_{2}=0.7, n^{\prime}=2.0\right.$, Eq. 5-22) 
The variation of $m^{\prime}$ with $\theta$ (Eq. 5-29) is shown in Fig. 5-15 as the solid curve. The original correlation dotted curve shown in the figure) significantly underestimates $m^{\prime}(\theta)$ (and thus $V_{d}$ ) at $50^{\circ} \leq \theta \leq 88^{\circ}$. This underestimation explains some of the error in Fig. 5-13.

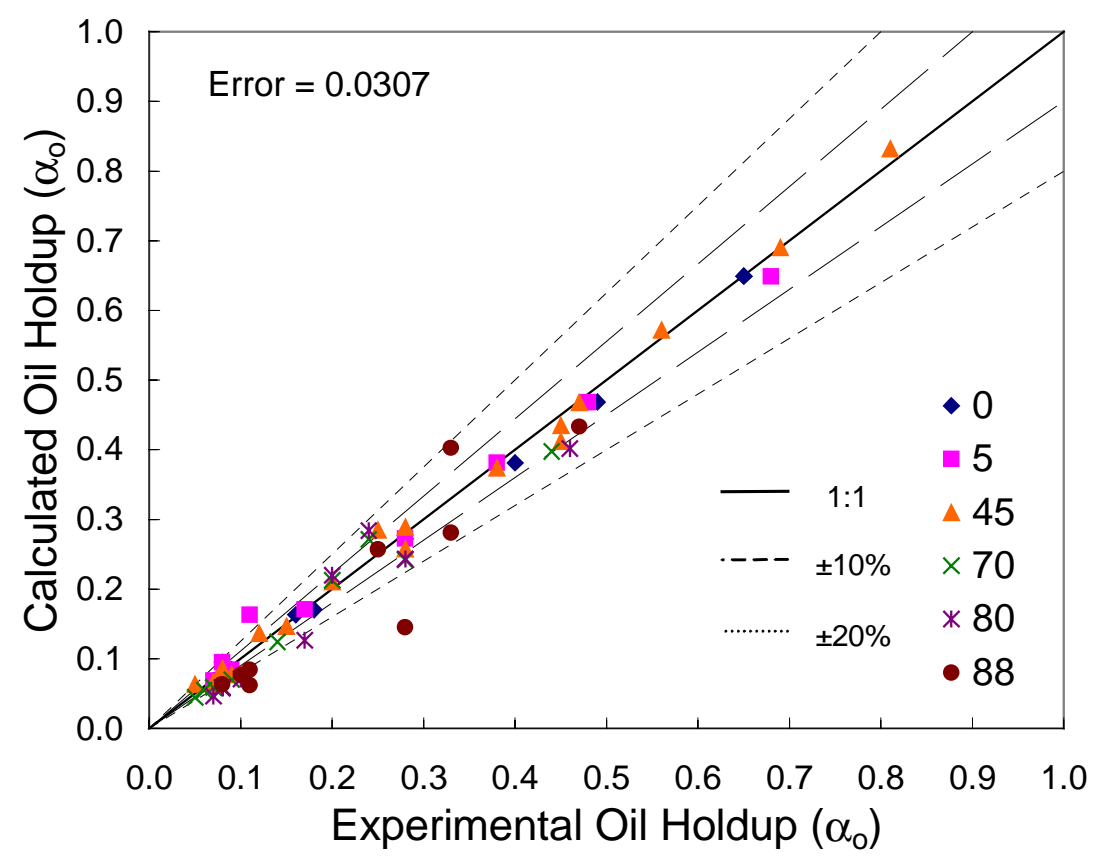

Figure 5-14: Predicted oil holdup using optimized parameters $\left(A^{\prime}=1.0, n^{\prime}=1.0\right.$, Eq. 5-28)

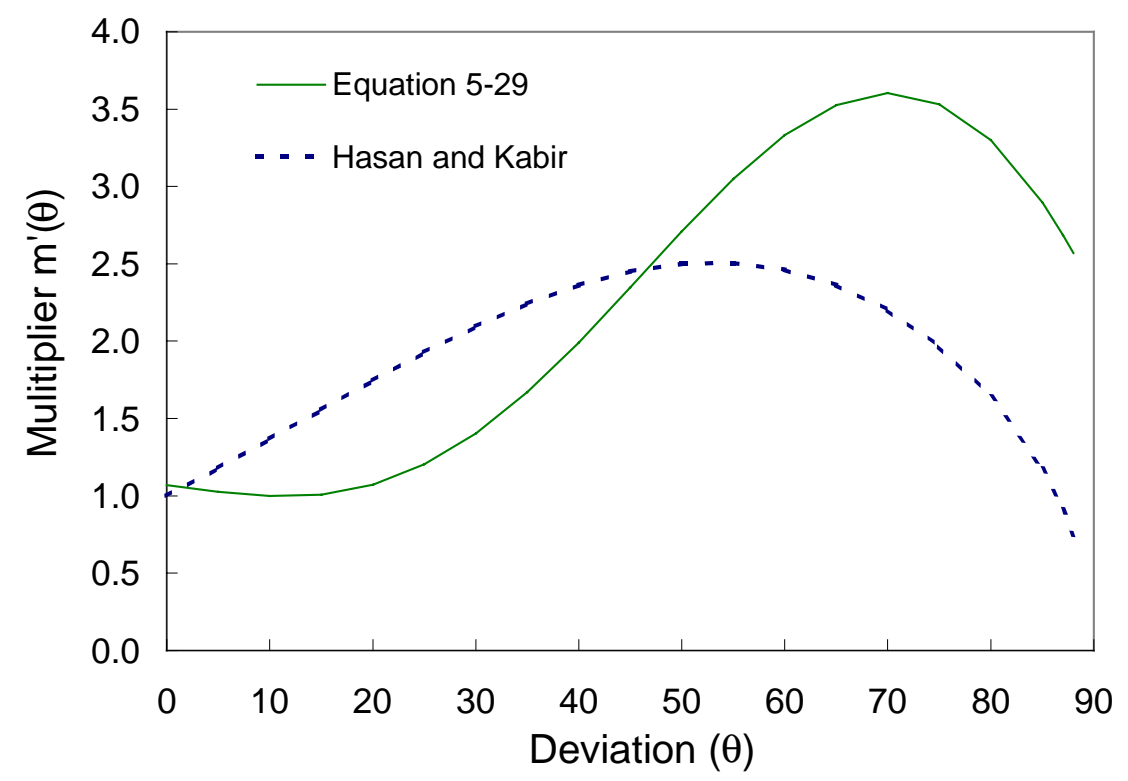

Figure 5-15: Deviation effect for oil-water system 


\subsection{Three-Phase Parameter Determination}

To model three-phase flow, we first apply a two-stage approach based purely on the twophase flow models. As discussed in the Introduction, we treat the system first as a gas-liquid flow to determine $\alpha_{g}$ and then model the liquid as an oil-water system to determine $\alpha_{o}$ and $\alpha_{w}$. We use the optimized parameters presented above for these calculations.

This relatively simple approach is well-suited for situations in which oil, water and gas flow as three-distinct layers; i.e., for stratified flow. It is also capable of modeling the separation of a three-phase mixture into pure phases of gas, oil and water through transient counter-current flow. However, it is known that, in many steady-state flows (Oddie et al. 2003), the effect of the third phase cannot be ignored. We now assess the accuracy of this treatment for our three-phase flow data.

\subsubsection{Application of Water-Gas Results to Three-Phase Flow}

We now consider the determination of $\alpha_{g}$ in three-phase systems. For this calculation, we view the system as a gas-liquid flow and apply our optimized two-phase water-gas data. In these optimizations, we use the experimental values of $\alpha_{o l}$ and $\alpha_{w l}$, where $\alpha_{o l}$ is the in situ volume fraction of oil in the liquid phase; i.e., $\alpha_{o l}=\alpha_{o} /\left(\alpha_{o}+\alpha_{w}\right)$ and similarly for $\alpha_{w l}$ $\left(\alpha_{w l}=1-\alpha_{o l}\right)$. These values are used to compute average liquid phase properties. Following the three-phase optimization results, we will apply the overall procedure using only the phase flow rates $\left(Q_{w}, Q_{o}, Q_{g}\right)$.

The results for $\alpha_{g}$ for inclinations over the range $0 \leq \theta \leq 88^{\circ}$, using the original parameters (Schlumberger GeoQuest, 2001), are shown in Fig. 5-16. We see a clear tendency toward overprediction over the entire range of experimental data $\left(\alpha_{g}<0.35\right)$. Most of the points are outside of the $20 \%$ relative error line.

We next apply our optimized gas-water parameters $\left(A=1.0, a_{1}=0.06, a_{2}=0.21\right.$, Eq. 5-27) to compute $\alpha_{g}$ for three-phase flow. The results, shown in Fig. 5-17, are quite reasonable, with the average error reduced to 0.0361 (from 0.0822 using the original parameters). Several points, however, continue to fall outside of the $\pm 20 \%$ range. 


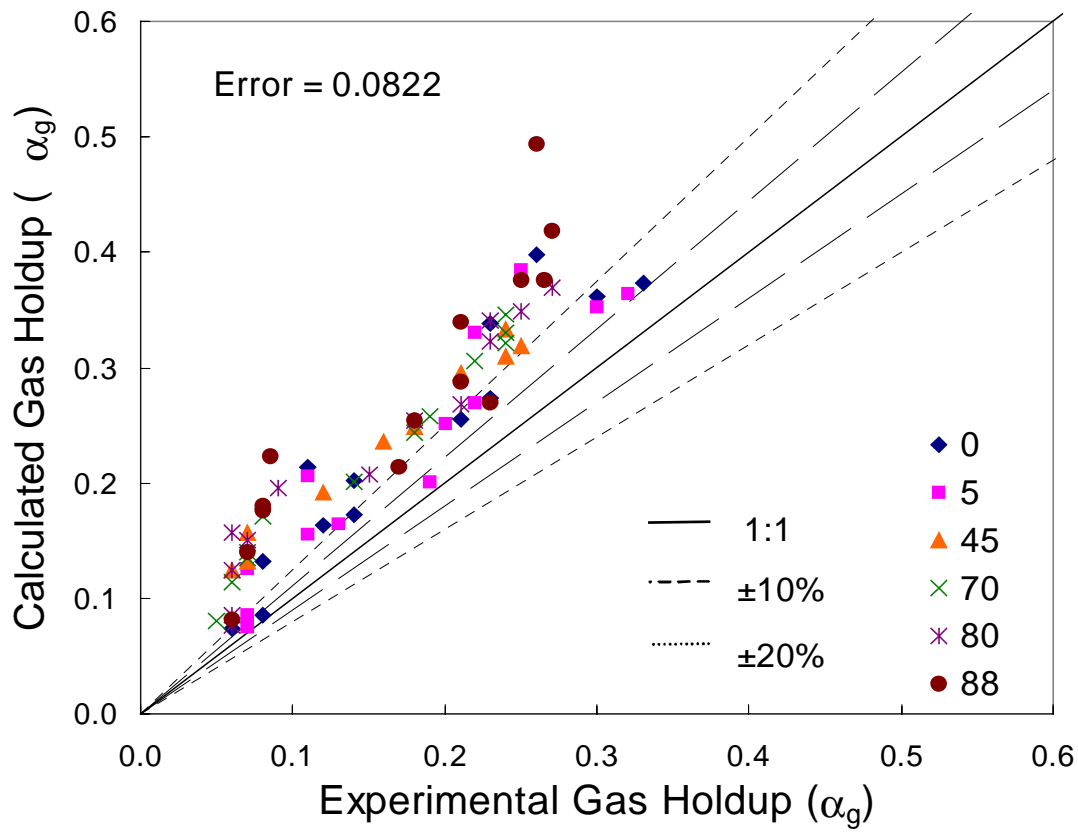

Figure 5-16: Three-phase gas holdup using original ECLIPSE parameters $\left(A=1.2, B=0.30, a_{1}=0.20, a_{2}=0.40\right.$, Eq. 5-22)

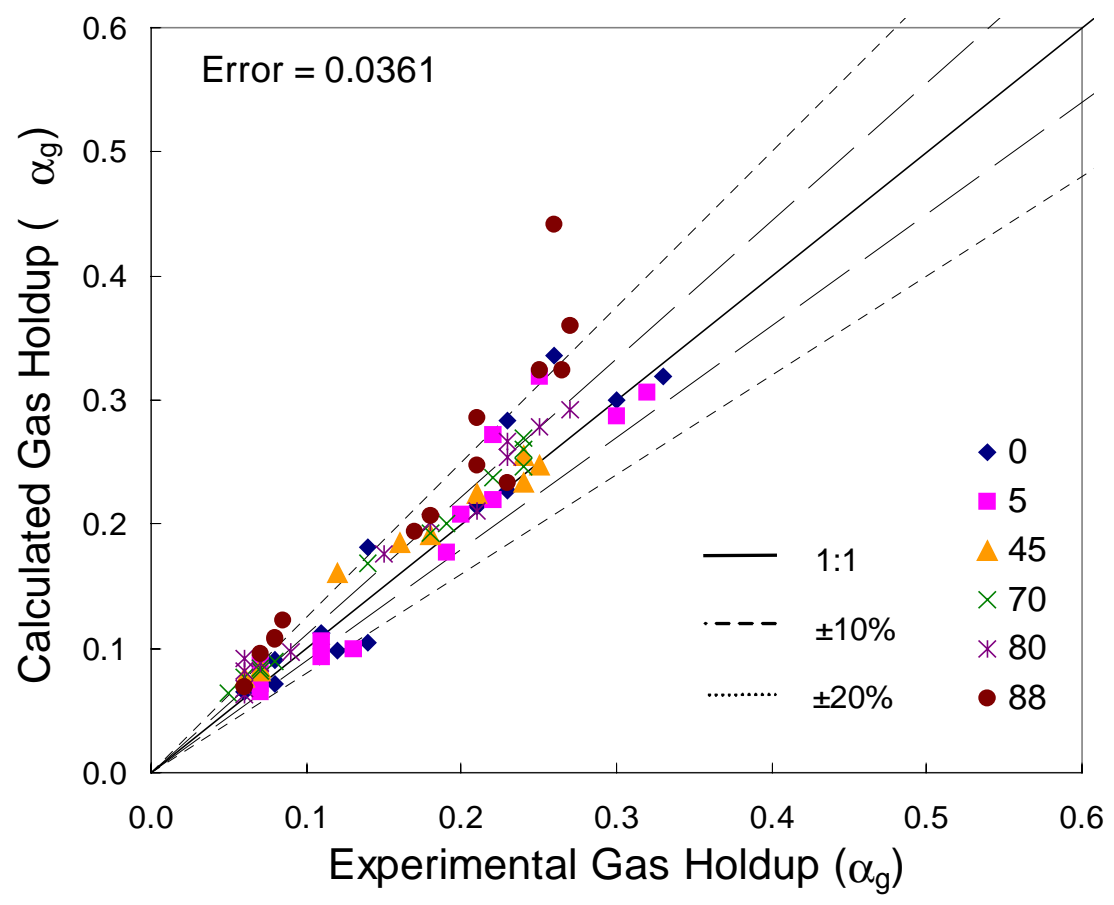

Figure 5-17: Three-phase gas holdup using two-phase optimized parameters $\left(A=1.0, a_{1}=0.06, a_{2}=0.21\right.$, Eq. 5-27) 
We obtained similar results for $\alpha_{g}$, but with slightly higher error (0.0385), using the twophase model with $A=1.2, B=0.6, a_{1}=0.06, a_{2}=0.12$, and $m(\theta)=1.27(\cos \theta)^{0.24}(1+\sin \theta)^{1.08}$. Similar levels of error were achieved with other sets of parameters (i.e., with $A=1.1$ ). This again indicates that our measurements of $\alpha_{g}$ can be matched to a reasonable degree of accuracy both with and without profile slip.

From Fig. 5-17, we conclude that the optimized water-gas flow parameters can be applied to estimate $\alpha_{g}$ in three-phase flow. The prediction from a direct optimization of the experimental $\alpha_{g}$ for the three-phase system would provide improved results, but the results from this simplified procedure are satisfactory. An advantage of this approach is that the three-phase flow model reduces to the two-phase model when one of the liquid phases disappears.

\subsubsection{Application of Oil-Water Results to Three-Phase Flow}

We now use the two-stage procedure to determine the oil and water fractions in the combined 'liquid' phase. Here we use $A^{\prime}=1.0, n^{\prime}=1.0$, and Eq. 5-29 to estimate the oil volume fraction in the liquid phase $\left(\alpha_{o l}\right)$.

The predictions for $\alpha_{o l}$ using the original parameters (Schlumberger GeoQuest, 2001) are shown in Fig. 5-18. Significant scatter is evident (error of 0.149), with some points overpredicted and others underpredicted, for all six deviations. Applying the optimized oilwater parameters to obtain $\alpha_{o l}$ for three-phase flow gives the results shown in Fig. 5-19. The error here is still very large (0.141) and most of the points are underpredicted. From these results we conclude that the optimized two-phase oil-water parameters are not suitable for the prediction of $\alpha_{o l}$.

The underprediction evident in Fig. 5-19 indicates that $V_{d}^{\prime}$ for three-phase flow is much lower than that for two-phase oil-water flow, except when the pipe is near horizontal $\left(88^{\circ}\right)$. It 
is worth reiterating that only $V_{d}^{\prime}$ affects these results, as there is no profile slip between oil and water $\left(C_{0}^{\prime}=1.0\right)$. The results suggest that additional effects arise in three-phase flows that must be specifically modeled.

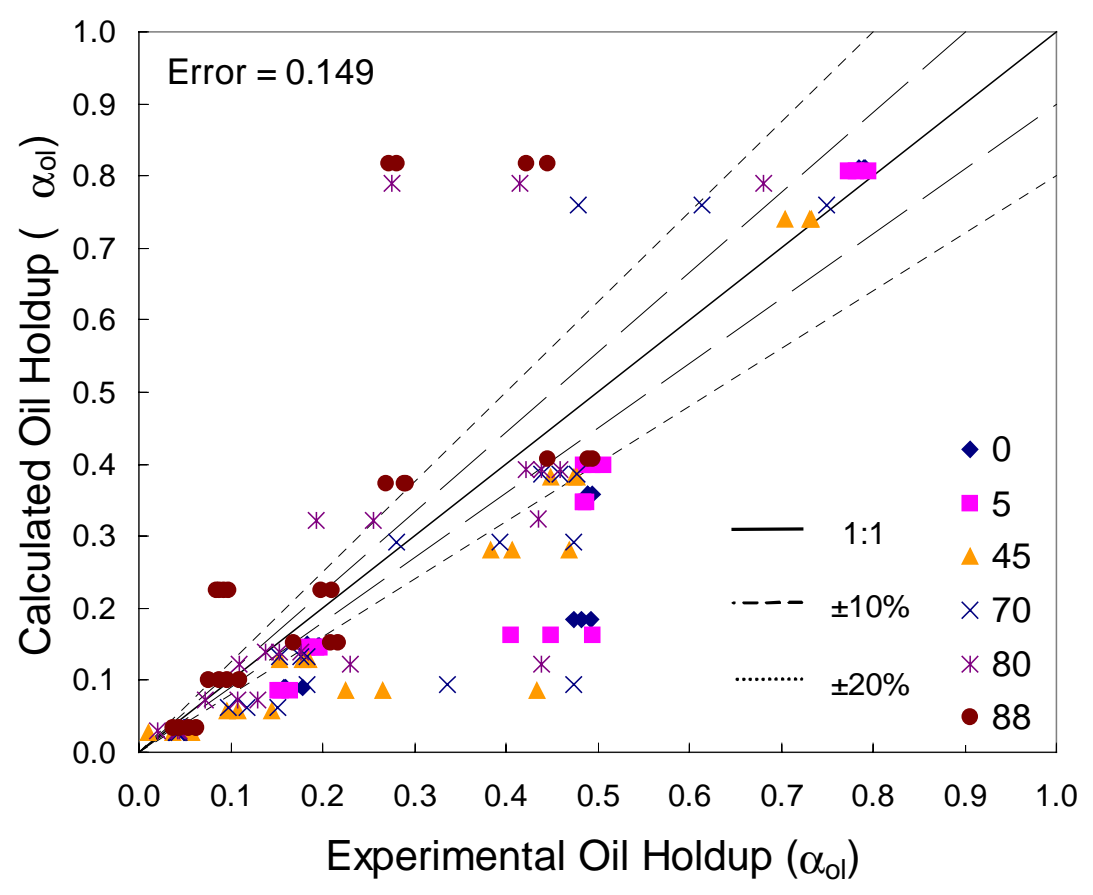

Figure 5-18: Three-phase oil holdup using original ECLIPSE parameters $\left(A^{\prime}=1.2, B_{1}^{\prime}=0.40, B_{2}^{\prime}=0.70, n^{\prime}=2.0\right.$, Eq. 5-22) 


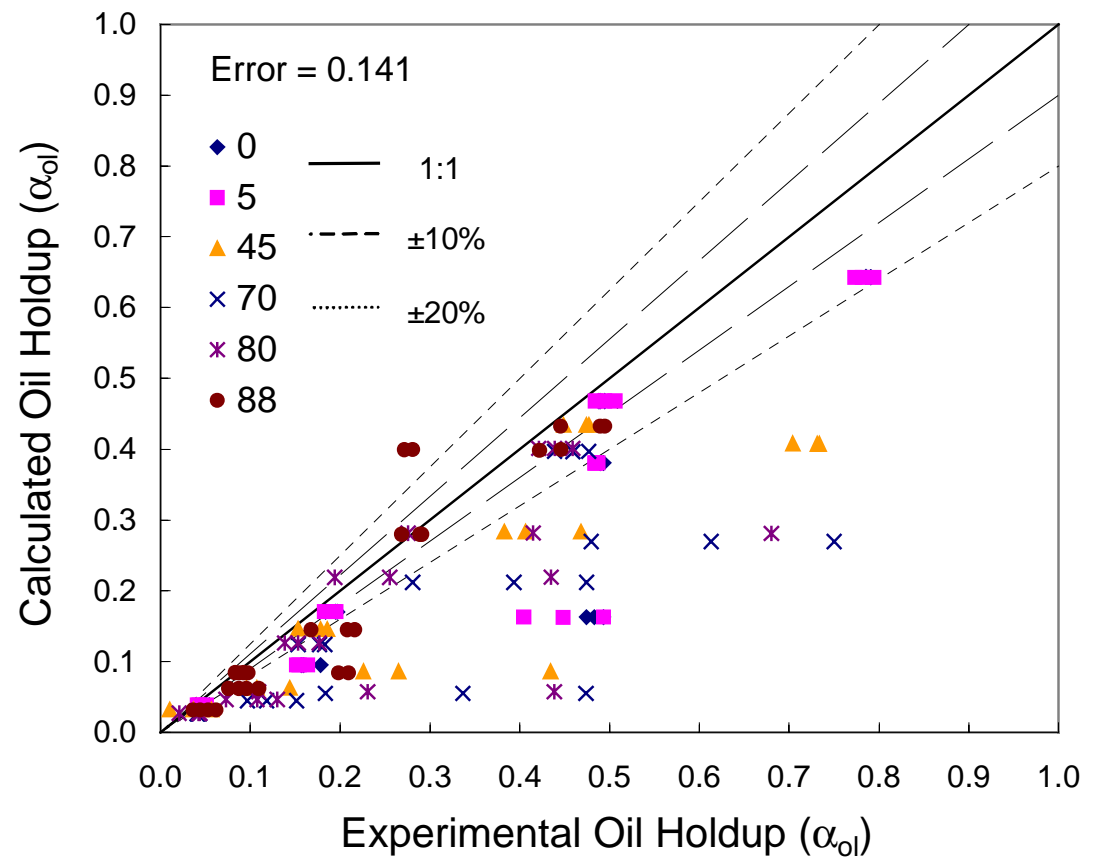

Figure 5-19: Three-phase oil holdup using two-phase optimized parameters $\left(A^{\prime}=1.0, n^{\prime}=1.0\right.$, Eq. 5-29)

The substantial reduction in slip between oil and water in three-phase flow is due to the effect of the gas. To illustrate the effect of gas on oil-water slip in three-phase flows, we now compare the no-slip $\alpha_{o l}$ (i.e., input oil volume fraction in the liquid phase, $C_{o l}$, given by $\left.Q_{o} /\left(Q_{o}+Q_{w}\right)\right)$ with the experimentally determined $\alpha_{o l}$ at different deviations. Fig. 5-20 shows these results for a vertical pipe. Here the experimental $\alpha_{o l}$ are in close agreement with the input volume fraction $C_{o l}$, indicating that there is essentially no slip between oil and water in these experiments. Thus, we can treat this particular three-phase flow system as a two-phase liquid-gas flow with the oil in situ fraction given by $\alpha_{o}=\left(1-\alpha_{g}\right) \frac{Q_{o}}{Q_{o}+Q_{w}}$.

This lack of slip between oil and water in three-phase flow is not, however, universal. To demonstrate this, we consider three-phase flow in a nearly horizontal pipe $\left(\theta=80^{\circ}\right)$. In the results for this case, shown in Fig. 5-21, we see significant slip between oil and water for most of the points, indicating there is much less gas effect than was evident in Fig. 5-20. It is also interesting to see that the points align in five horizontal groups. Each group corresponds 
to a set of experimental tests with particular oil and water flow rates (i.e., the same input fractions). As gas flow rate increases, the experimental $\alpha_{o l}$ approach $C_{o l}$. This indicates that gas disrupts the slip between oil and water when there is sufficient gas in the system.

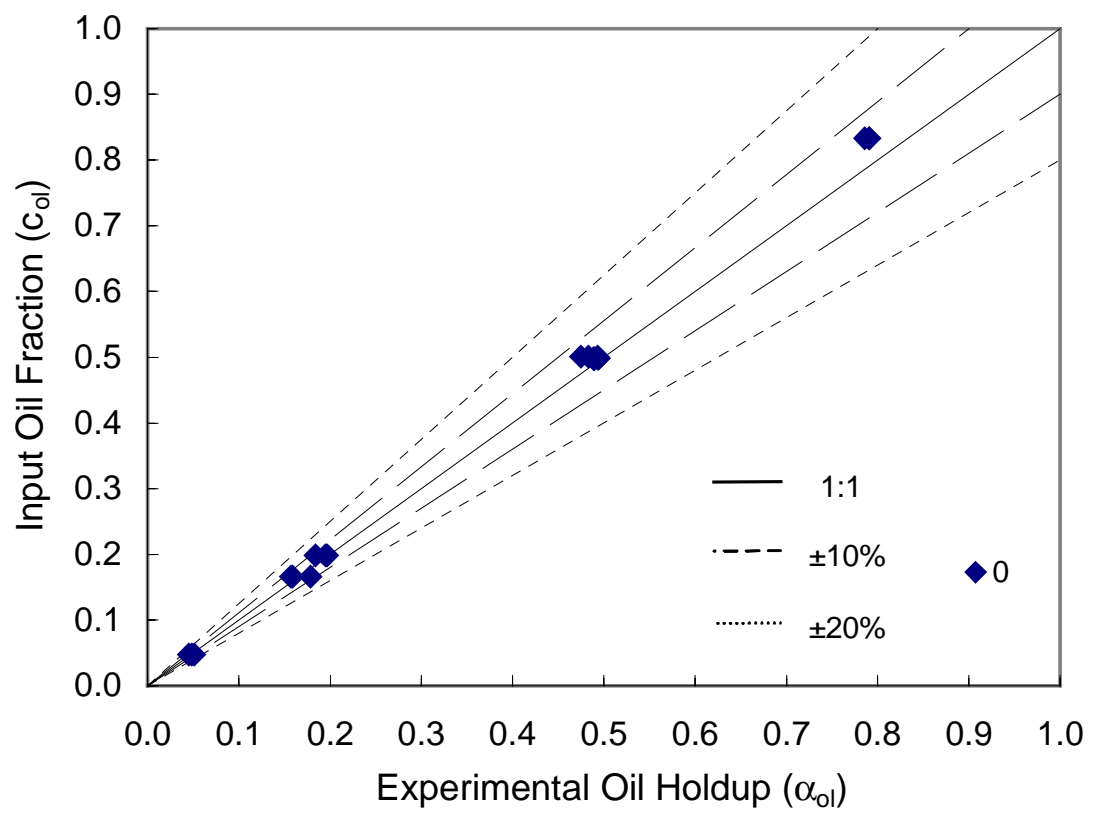

Figure 5-20: Oil holdup in liquid for oil-water-gas system for $\theta=0^{\circ}$

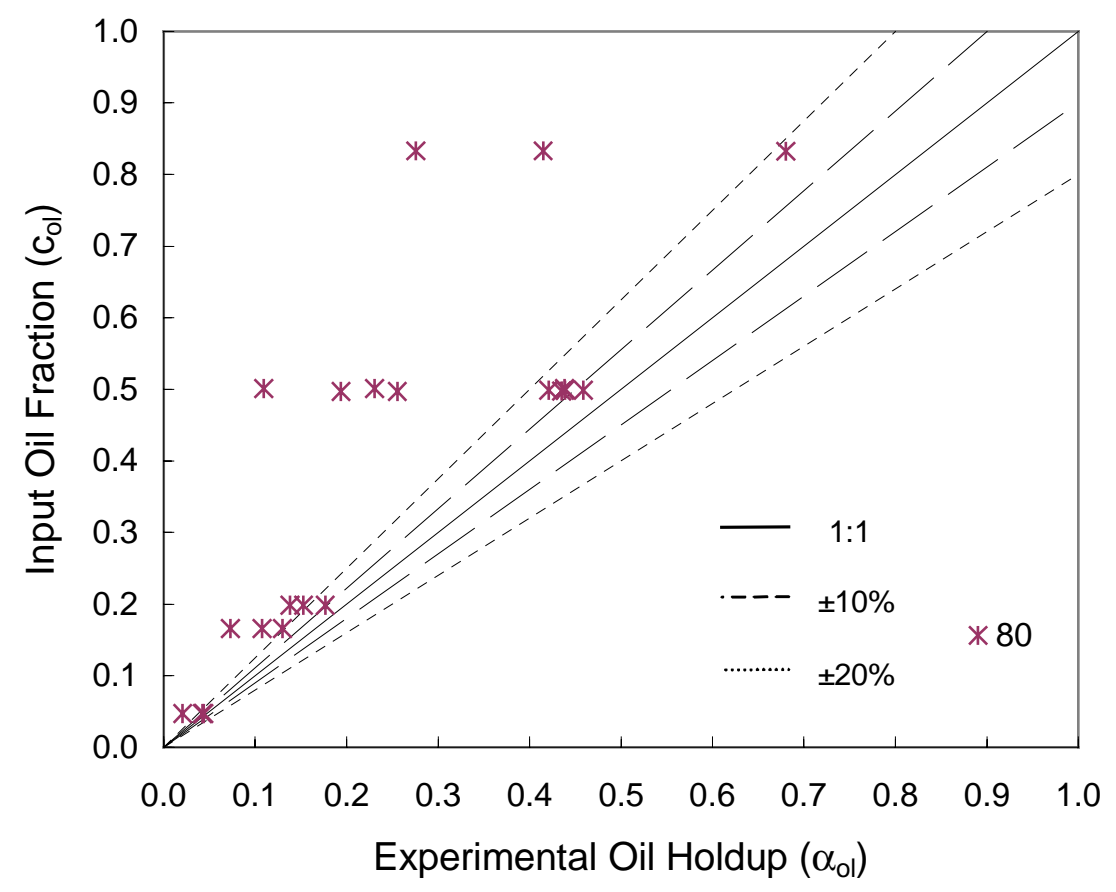

Figure 5-21: Oil holdup in liquid for oil-water-gas system for $\theta=80^{\circ}$ 
It is apparent from Figs. 5-20 and 5-21 that, in some three-phase systems, there is no slip between oil and water, though in other cases there is slip between oil and water. The effect of gas on oil-water flow is complicated, though it largely depends on the pipe deviation. We will now extend our drift-flux model to capture this important effect.

\subsubsection{Oil-Water Model in Three-Phase Flow}

Our goal here is to develop a unified model to predict oil and water holdup in three-phase flow systems. By unified model, we mean one that reduces to the two-phase oil-water model when there is no gas present in the system.

In order to develop this unified model, we first directly determine optimized parameters for oil and water within three-phase flow. In this optimization, we fix $A^{\prime}$ and $n^{\prime}$ to their

optimum values for two-phase oil-water flow and optimize only the $V_{d}^{\prime}$ multiplier $m^{\prime}(\theta)$. The resulting three-phase $m^{\prime}(\theta)$ is shown in Fig. 5-22 (square points). These results deviate considerably from the two-phase $m^{\prime}(\theta)$ curve (Eq. 5-29), shown in the figure as the solid curve. It is apparent that $m^{\prime}(\theta)$ for three-phase flow is close to zero for vertical flow and is very small for $\theta=5^{\circ}$, indicating there is no slip between oil and water at these two deviations. We observe that $m^{\prime}(\theta)$ then increases with $\theta$, with a sharper increase for $\theta>70^{\circ}$. The relatively high $m^{\prime}(\theta)$ values for highly deviated pipes are due to gas segregation. In fact, most of the flow patterns observed for deviations of $80^{\circ} \leq \theta<90^{\circ}$ were elongated bubble flows with gas segregated from the liquid (see Oddie et al., 2003). 


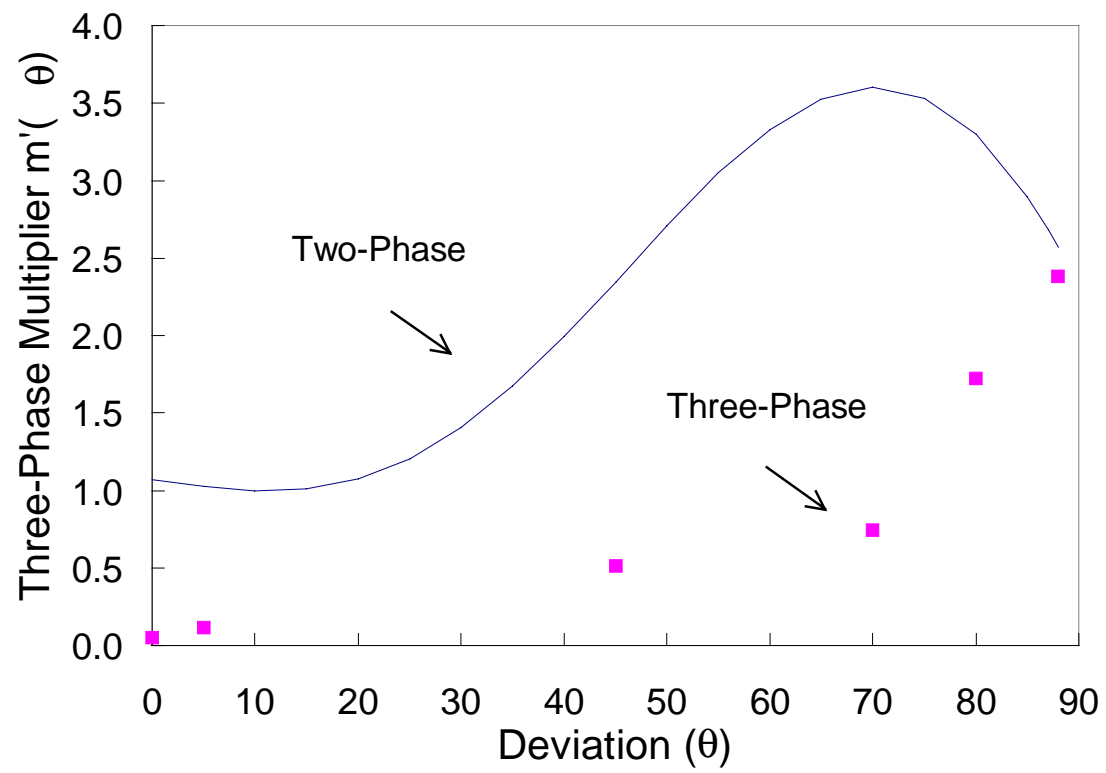

Figure 5-22: Deviation effect for oil-water in three-phase system

Based on the results in Fig. 5-22, we propose the following unified model:

$$
m_{3-\text { phase }}^{o w}=m_{2-\text { phase }}^{\text {ow }} m_{g}
$$

where $m_{g}$ captures the gas effect. Since the two-phase function $m_{2-\text { phase }}^{o w}$ depends only on $\theta$, $m_{g}$ must depend on both $\theta$ and $\alpha_{g}$ to provide the required functionality in $m_{3-p h a s e}^{o w}$. We prescribe the following simple functional form for $m_{g}$ :

$$
\begin{array}{rlrlrl}
m_{g}\left(\theta, \alpha_{g}\right) & =1-\frac{\alpha_{g}}{a_{3}(\theta)} & \text { when } & \alpha_{g}<a_{3} \\
& =0 & & \text { when } & \alpha_{g} \geq a_{3}
\end{array}
$$

Note that we have introduced a new parameter, $a_{3}(\theta)$. When the gas holdup decreases to zero, the model reverts smoothly to the two-phase oil-water model. However, when the gas holdup exceeds $a_{3}$, the oil-water slip vanishes. The model behaves linearly between these two limits. We note that $a_{3}$ should technically also be a function of velocity, as the results in Fig. 5-21 suggest. However, we do not have sufficient data at high $\alpha_{g}$ to model this effect. Were we to include the velocity effect, $a_{3}$ would be smaller for near horizontal flows when gas is 
entrained in liquid. This would, however, introduce only a small correction since $V_{d}^{\prime}$ is less important at higher flow velocities.

The equation for $V_{d}^{\prime}$ for oil and water in a three-phase system is now given by:

$$
V_{d}^{\prime}=1.53 V_{c}^{\prime}\left(1-\alpha_{o l}\right) m_{2-p h a s e}^{o w}(\theta) m_{g}\left(\theta, \alpha_{g}\right)
$$

This equation reduces to the result for two-phase oil-water flow when $\alpha_{g} \rightarrow 0$, indicating that the two and three-phase flow models are unified.

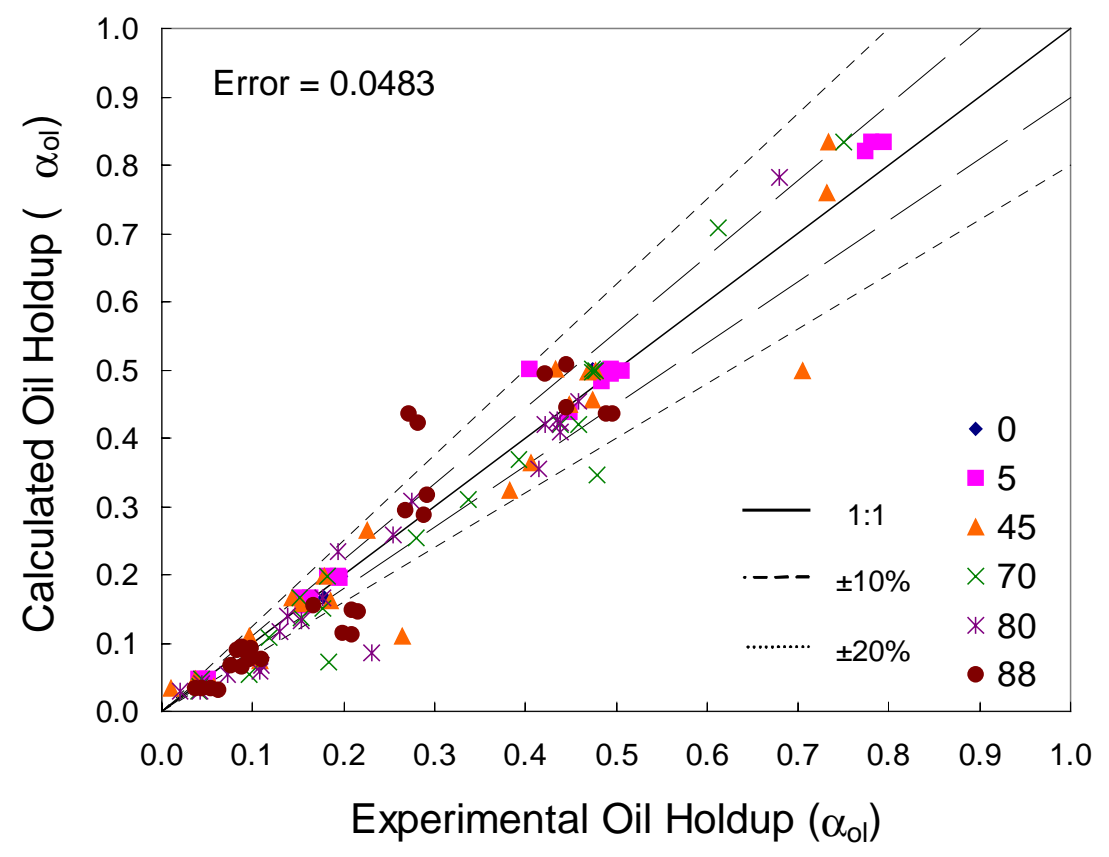

Figure 5-23: Predicted oil holdup using the new three-phase model

Fig. 5-23 shows the results for $\alpha_{o l}$ obtained using this new model. The error here is about one third of that obtained with the original parameters or with the optimized two-phase parameters. Fig. 5-24 shows the gas effect in terms of the variation of $a_{3}$ with deviation. We see that, from vertical up to $70^{\circ}$ deviation, only a small amount of gas $\left(\alpha_{g} \approx 0.1\right)$ is required to eliminate the slip between oil and water. But, for near horizontal flow, gas has a much smaller effect on the oil-water slip. We interpret this as being due to the gas being segregated 
from the liquid in our experiments at near horizontal deviations. The points in Fig. 5-24 can be approximated by the following equation (this is the curve shown in the figure):

$$
a_{3}(\theta)=0.017 \exp \left(\theta^{3.28}\right)
$$

where $\theta$ is in radians. Note that this equation is valid from vertical up to $88^{\circ}$ deviation, where $a_{3}(\theta) \leq 1$.

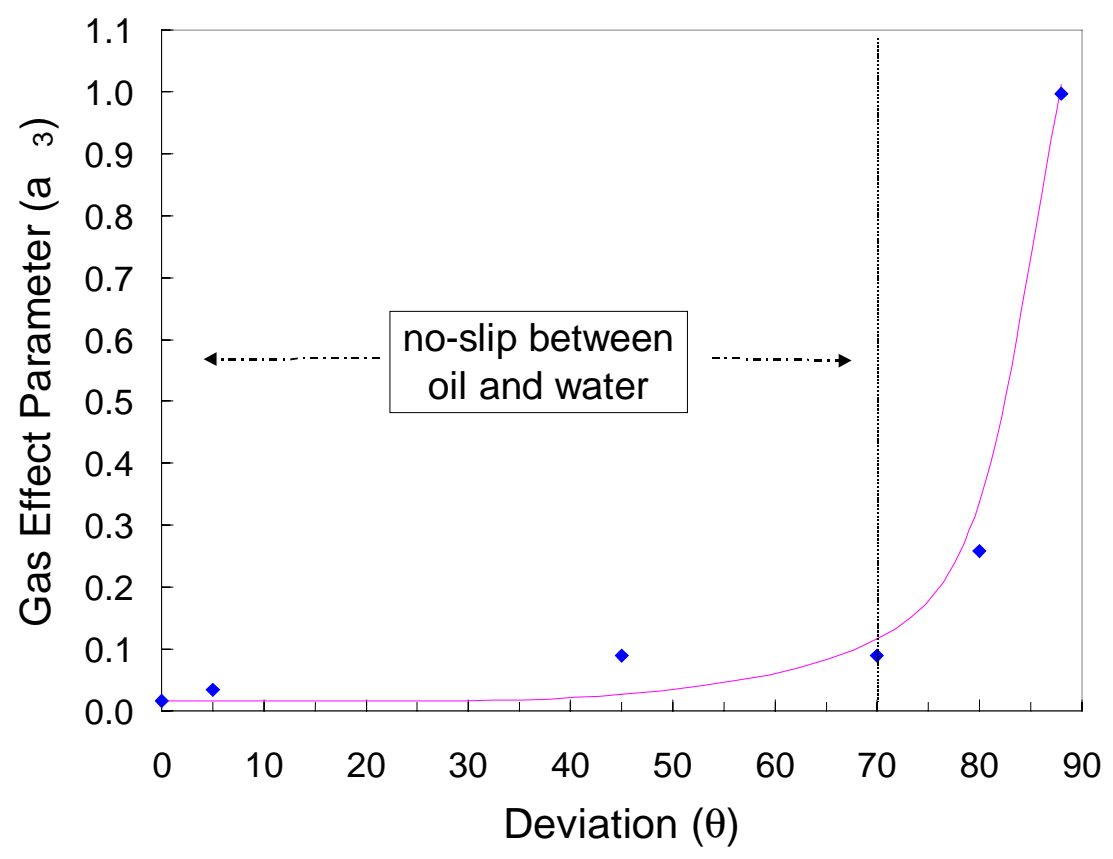

Figure 5-24: Gas effect on oil-water in three-phase system

\subsubsection{Holdup Prediction from Volumetric Flow Rates}

In the determination of $\alpha_{g}$ in the optimized model developed above, we require knowledge of $\alpha_{o l}$ and $\alpha_{w l}$ (in order to compute average liquid phase properties). However, the determination of $\alpha_{o l}$ and $\alpha_{w l}$ requires us to know $\alpha_{g}$ (to compute the effect of gas on oilwater slip). Thus, given only the phase flow rates $\left(Q_{g}, Q_{o}, Q_{w}\right)$, and not $\alpha_{o l}$ and $\alpha_{w l}$, the calculation of holdups must be accomplished iteratively. Our procedure is as follows. We first compute $\alpha_{g}$ using liquid phase properties approximated under the assumption of no slip between the oil and water phases (i.e., $\left.\alpha_{o l}=C_{o l}=Q_{o} /\left(Q_{o}+Q_{w}\right)\right)$. Then, using this $\alpha_{g}$, we 
compute $\alpha_{o l}$ and $\alpha_{w l}$ using our new three-phase model. We then recompute $\alpha_{g}$ using the updated values for $\alpha_{o l}$ and $\alpha_{w l}$. This procedure is continued until the holdups no longer change. In cases with no slip between oil and water, only one iteration is required; in cases with oil-water slip, two or three iterations are typically required.

The accuracy of the predicted holdups is fairly close to that achieved in the optimized results shown in Figs. 5-17 and 5-23. Specifically, the average error in $\alpha_{g}$ is 0.0379 (compared to 0.0361 in Fig. 5-17) and the average error in $\alpha_{o l}$ is 0.0643 (compared to 0.0483 in Fig. 5-23). The error in $\alpha_{o l}$ increases somewhat because of the overprediction of $\alpha_{g}$ for near-horizontal flows (evident in Fig. 5-17). This in turn causes some of the oil-water flows to be treated with no slip when there is in fact some slip between the oil and water. The accuracy of the predicted $\alpha_{o l}$ could be improved if we enhanced the model for $\alpha_{g}$ directly for three-phase systems. This does not appear to be necessary, however, as the level of accuracy of the predicted holdups is quite acceptable.

\subsection{Development of a Multi-Segment Well Simulator - MSWell}

\subsubsection{Introduction}

The modeling of advanced (multilateral, horizontal, smart) wells requires more sophisticated well models than are required for conventional wells. Models for advanced wells must allow pressure, flow rate and fluid compositions to vary with position in the wellbore, and enable different fluid streams to co-mingle at branch junctions (Holmes, 2003). This can be accomplished by discretizing the well into segments and solving the mass balance equations and pressure drop equation for each segment.

A one dimensional, three-phase, compressible, fully implicit well simulator (MSWel1) was developed. The fully implicit solution procedure is necessary to maintain stability for coupling with the reservoir simulator. MSWell computes the holdups and velocities of the phases in each segment, as well as the pressure profile along the wellbore, under both steady state and transient conditions. One of the major purposes for developing such a simulator is for implementation into GPRS for modeling advanced wells. The MSWell model can also be 
used for optimizing the parameters of the drift-flux model to obtain better agreement with the steady state and transient wellbore flow data. The initial implementation of MSWell is very similar to the multiphase flow model in ECLIPSE (Schlumberger GeoQuest, 2001).

\subsubsection{Model Formulation}

The wellbore is divided into a one-dimensional multi-segment system. The system contains four primary variables in each segment at each time step. The variables are gas holdup, $\alpha_{g}$, oil holdup, $\alpha_{o}$, mixture velocity, $V_{m}$ and pressure, $P$. The water holdup, $\alpha_{w}$, is also an unknown, but it can be obtained from the constraint $\alpha_{g}+\alpha_{o}+\alpha_{w}=1$. For a compressible system, the unknowns will change from segment to segment even for steady state due to pressure variations.

The governing equations for the system are the mass balance equation for each phase and a pressure equation. These four equations are solved to determine the four primary unknowns. The general mass balance for phase $p$ (assuming that gas does not dissolve in the liquid phases) is as follows:

$$
\frac{\partial}{\partial t}\left(\rho_{p} \alpha_{p}\right)-\frac{\partial}{\partial x}\left(\rho_{p} V_{s p}\right)=\tilde{m}_{p}
$$

where $t$ is time, $\rho_{p}$ is the phase density, $x$ is position along the wellbore, $V_{s p}$ is the superficial velocity of phase $p$, and $\tilde{m}_{p}$ is the inflow/outflow mass flow rate per unit volume of the segment due to exchange with the reservoir.

The density can be expressed as

$$
\rho_{p}=b_{p} \rho_{p}{ }^{S T C}
$$

where $b_{p}=1 / B_{p}$ and $B_{p}$ is the formation volume factor, and $\rho_{p}{ }^{S T C}$ is the phase density under standard conditions. The phase superficial velocity is defined as

$$
V_{s p}=\frac{Q_{p}}{A_{w}}
$$

where $A_{w}$ is the cross-sectional area of the well and $Q_{p}$ is the phase volumetric flow rate. 
Dividing Eq. 5-34 by $A_{w}$ and $\rho^{S T C}$ and introducing a finite difference discretization, the general mass balance equation is

$$
\frac{\Delta x}{\Delta t}\left[\left(b_{p} \alpha_{p}\right)^{n+1}-\left(b_{p} \alpha_{p}\right)^{n}\right]-\left[\left(b_{p} V_{s p}\right)_{i n}^{n+1}-\left(b_{p} V_{s p}\right)_{o u t}^{n+1}\right]=\left(\frac{\tilde{m}_{p}}{A \rho_{p}^{S T C}}\right)
$$

where in and out are the boundaries of the segment and $n$ is the time step. Eq. 5-37 is written for $p=g, o$, and $w$ (gas, oil and water). In the gas balance, $b_{g}$ is obtained using the real gas law

$$
b_{g}=\frac{M}{c_{g} R T} \frac{1}{\rho^{S T C}} P
$$

where $M$ is the molecular weight, $c_{g}$ is the gas compressibility factor, $R$ is the gas constant, and $T$ is the temperature. For the liquid phases, the following expression is used:

$$
b_{l}=1+c_{l}\left(P-P^{S T C}\right)
$$

where $l$ stands for liquid (oil or water), $c$ is the compressibility factor and $P^{S T C}$ is the pressure at standard conditions.

For the pressure loss equation, we have two options. The first option is to apply the pressure equation used in ECLIPSE (Schlumberger GeoQuest, 2001). This pressure equation has three components, the hydrostatic pressure, the frictional pressure, and the acceleration component:

$$
\Delta P=\Delta P_{h}+\Delta P_{f}+\Delta P_{a}
$$

The hydrostatic pressure difference between two segments is given by

$$
\Delta P_{h}=\frac{\left(\rho_{m}\right)_{i}+\left(\rho_{m}\right)_{i+1}}{2} g \Delta x \cos \theta
$$

where $i$ is the segment number, $g$ is the gravitational acceleration, $\Delta x \cos \theta$ is the height difference between the segments, $\theta$ is the inclination of the well from vertical and $\rho_{m}$ is the mixture density defined as

$$
\rho_{m}=\alpha_{g} \rho_{g}+\alpha_{o} \rho_{o}+\alpha_{w} \rho_{w}
$$

The frictional pressure component between two segments is: 


$$
\Delta P_{f}=\frac{2 f_{t p} \rho_{m} V_{m}^{2}}{D} \Delta x
$$

where $f_{t p}$ is the friction factor and the Haaland (1981) correlation for single phase flow is used to obtain this parameter:

$$
f_{t p}^{-0.5}=-3.6 \log \left[\frac{6.9}{\operatorname{Re}}+\left(\frac{\varepsilon}{3.7 D}\right)^{1.11}\right]
$$

where $\varepsilon$ is the pipe roughness and $R e$ is the Reynolds number defined as

$$
R e=\frac{\rho_{m} V_{m} D}{\mu_{m}}
$$

with $\mu_{m}=\alpha_{g} \mu_{g}+\alpha_{o} \mu_{o}+\alpha_{w} \mu_{w}$. The pressure component due to acceleration is given by:

$$
\Delta P_{a}=\frac{2 q_{i m} \rho_{m} V_{m}}{A_{w}} \Delta x
$$

where $q_{i m}$ is the volumetric flow rate of the mixture entering the segment.

The other option for the pressure equation is to use Ouyang's (1998) model. In this model, the total pressure gradient is decomposed into four parts:

$$
\Delta P=\Delta P_{h}+\Delta P_{f}^{\prime}+\Delta P_{a}^{\prime}+\Delta P_{e}
$$

where $\Delta P_{e}$ is the accelerational pressure gradient caused by fluid expansion. Among the other three components, only the hydrostatic pressure drop is calculated exactly the same as in the ECLIPSE pressure model (Eq. 5-41). For a complete description of this model, see Ouyang (1998).

The nonlinear system of equations is solved using Newton's method. Pressure can be fixed at any point. This pressure value is honored by iterating on the inlet pressure until the specified value is satisfied.

\subsubsection{Validation of MSWel1}

To validate the MSWell model, we compared the results from MSWell and ECLIPSE for gas-water, oil-water, and oil-water-gas systems. The comparisons were performed for both 
steady-state and transient flow (after shut-in) using a 50-segment wellbore of total length 11 $\mathrm{m}$. We expect close agreement since the formulations are very similar.

For a steady-state comparison, we use an example involving three-phase oil-water-gas flow. For this case, $Q_{o}=10.0 \mathrm{~m}^{3} / \mathrm{h}, Q_{w}=10.1 \mathrm{~m}^{3} / \mathrm{h}, Q_{g}=6.2 \mathrm{~m}^{3} / \mathrm{h}$, and $\theta=5^{\circ}$. The comparison for pressure prediction is shown in Fig. 5-25. The pressure along the wellbore decreases from about 2.4 bar to 1.5 bar almost linearly. This is because the hydrostatic pressure gradient dominates the total $\Delta P$ for $5^{\circ}$ deviation from vertical. It is seen that the prediction from MSWell matches the ECLIPSE result very closely. Fig. 5-26 shows the comparison for gas holdup. We see that gas expands along the wellbore as a result of pressure decreasing from the bottom to the top as shown in Fig. 5-25. Again, the gas holdup prediction by MSWell is very close to the ECLIPSE results.

For transient flow, we consider an oil-water flow. In this case, we have the same input of oil and water $\left(Q_{o}=10.0 \mathrm{~m}^{3} / \mathrm{h}, Q_{w}=10.1 \mathrm{~m}^{3} / \mathrm{h}\right)$ and $\theta=45^{\circ}$. Fig. 5-27 displays how the oil holdup of each segment evolves with time after shut-in. The process ends when oil and water are fully separated. In Fig. 5-27, each curve represents the $\alpha_{o}$ of each segment. All of the curves start at the same point $\left(\alpha_{o}=0.28\right)$, which corresponds to the steady-state $\alpha_{o}$. From Fig. 5-27, we see that the top segments gradually fill with oil while the bottom segments gradually fill with water as time proceeds. The segment that takes the longest time to settle is the segment containing the interface between oil and water. Comparing MSWell results (a) and ECLIPSE results (b), the match is seen to be very good. These results demonstrate that the MSWell implementation is performing as expected. In future work, this model will be implemented into GPRS. 


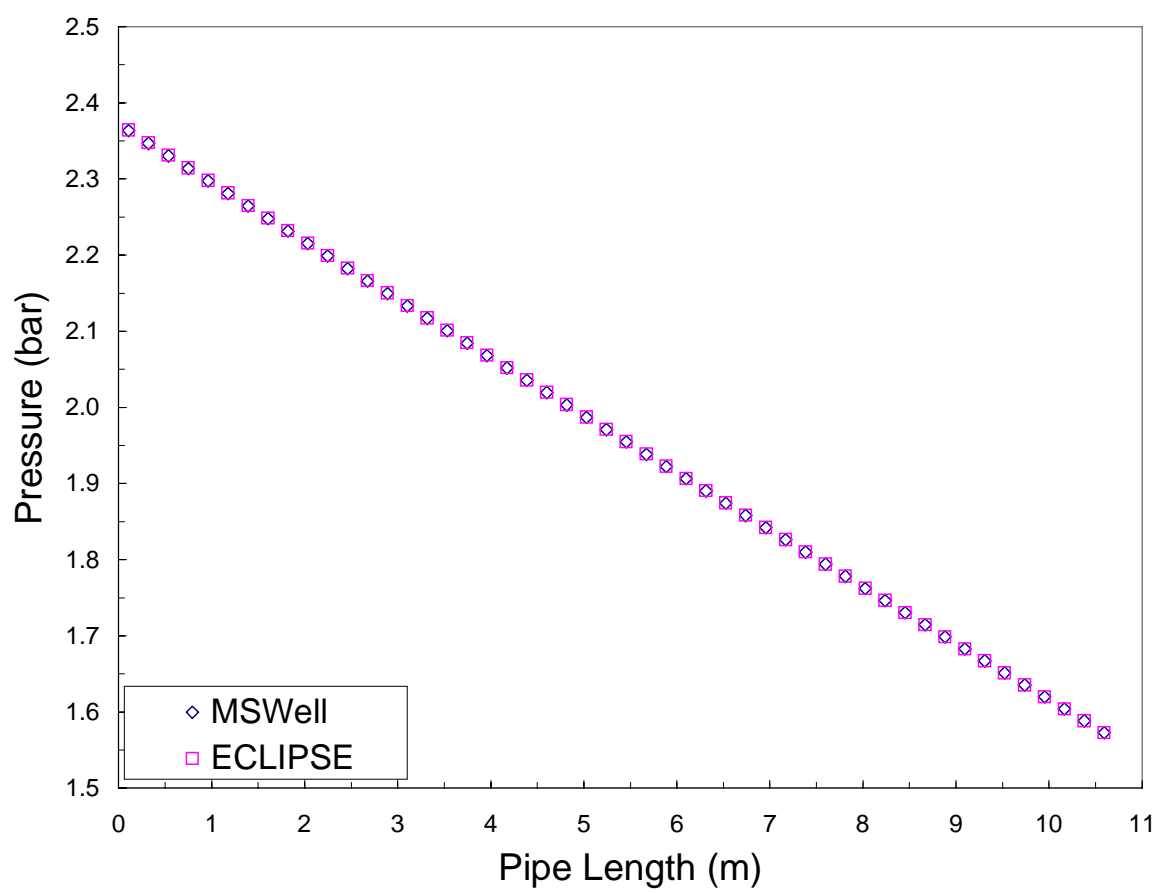

Figure 5-25: Pressure variation for an oil-water-gas system

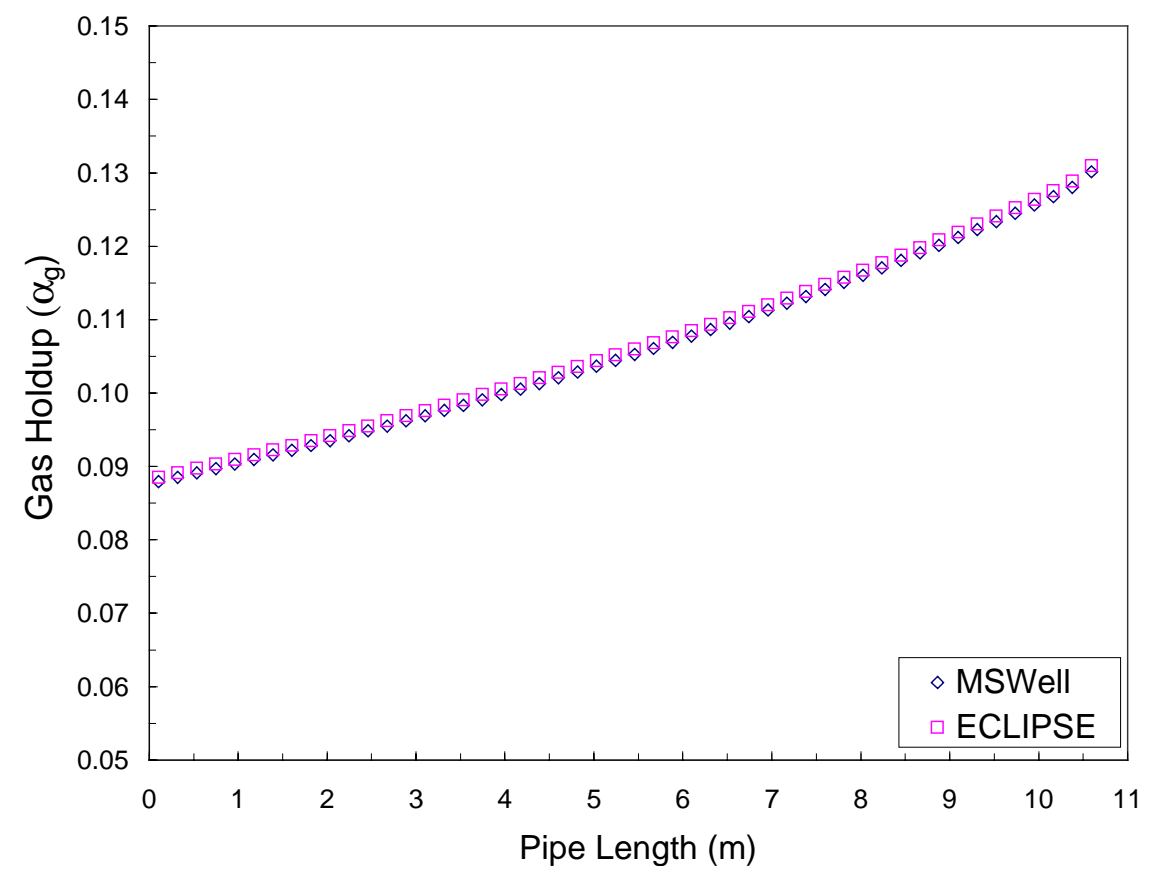

Figure 5-26 Gas holdup for an oil-water-gas system $\left(\theta=5^{\circ}, Q_{0}=10.0 \mathrm{~m}^{3} / \mathrm{h}, \mathrm{Q}_{\mathrm{w}}=10.1 \mathrm{~m}^{3} / \mathrm{h}, \mathrm{Q}_{\mathrm{g}}=6.2 \mathrm{~m}^{3} / \mathrm{h}\right)$ 
(a) MSWell Results

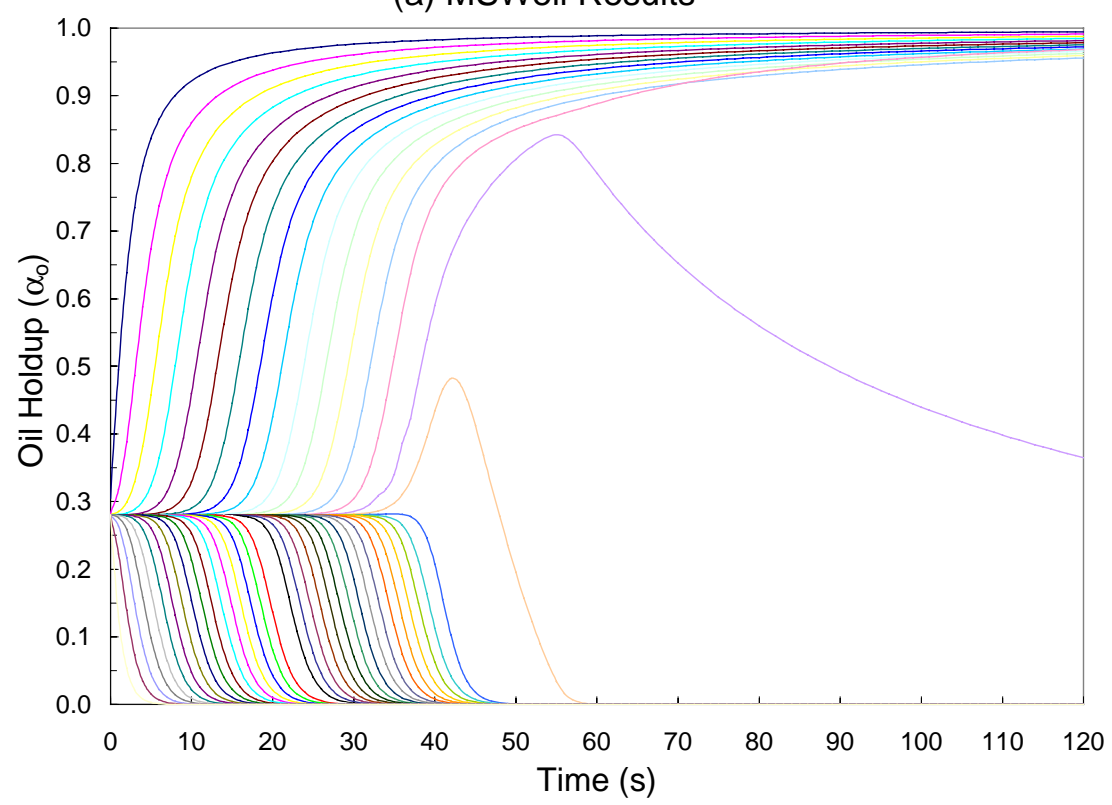

(b) ECLIPSE Results

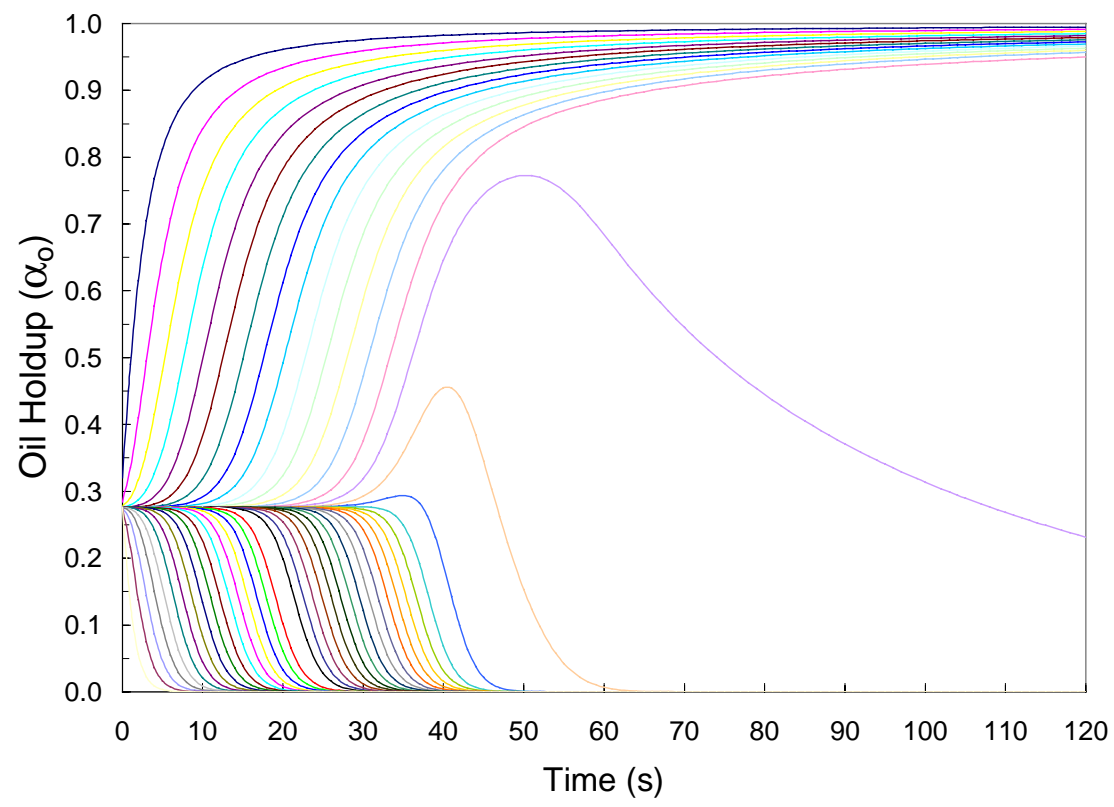

Figure 5-27: Transient oil holdup profiles for an oil-water system $\left(\theta=45^{\circ}, Q_{o}=10.0 \mathrm{~m}^{3} / \mathrm{h}, \mathrm{Q}_{\mathrm{w}}=10.1 \mathrm{~m}^{3} / \mathrm{h}\right)$ 


\subsection{Conclusions}

The drift-flux model is well suited for use in reservoir simulators because it is relatively simple and because it provides a continuous and differentiable representation of multiphase flow in pipes and wells. In this work, we used recently collected experimental data for largediameter pipes to determine drift-flux parameters for wellbores and pipes. A multiphase wellbore flow model, MSWel1, was also developed. The following conclusions can be drawn from this study:

- Drift-flux parameters determined from experiments using small-diameter pipes (2 inch or less) may not be appropriate for use in models of flow in large-diameter wellbores.

- Drift-flux descriptions with optimized parameters can accurately describe flow in largediameter pipes for water-gas, oil-water and oil-water-gas systems. Optimized parameters can be obtained using a unified optimization approach, which yields parameters applicable for the entire data range including all 6 deviations.

- The optimized parameters from two-phase water-gas systems can be applied to estimate gas holdup in three-phase flow.

- A small amount of gas $\left(\alpha_{g} \approx 0.1\right)$ in three-phase flow acts to eliminate the slip between oil and water for deviation up to $70^{\circ}$ from vertical. For near horizontal flows, gas $\left(\alpha_{g} \leq 0.35\right)$ affects the oil-water slip much less because the gas is separated from the oil-water mixture. A new gas effect parameter $\left(a_{3}\right)$ was introduced to capture the reduction of oil-water slip as a function of the gas holdup and the deviation.

- MSWe11, a multi-segment wellbore flow model, was developed to describe the local flowing conditions in advanced wells. The model will be implemented into GPRS to model multiphase flow in wellbores and surface facilities.

\subsection{Nomenclature}

$a_{1} \quad$ drift velocity ramping parameter

$a_{2} \quad$ drift velocity ramping parameter

$a_{3} \quad$ gas effect parameter 
A profile parameter term, value in bubble/slug regimes for liquid-gas flows

$A^{\prime} \quad$ profile parameter term for oil-water flows

$A_{w} \quad$ cross-sectional area of the well, $\mathrm{ft}^{2}$

$b \quad$ inverse formation factor $(b=1 / B)$

$B$ profile parameter term, gas volume fraction at which $C_{0}$ begins to reduce, formation factor

$B_{1} \quad$ profile parameter term, oil volume fraction at which $C_{0}^{\prime}$ begins to reduce

$B_{2} \quad$ profile parameter term, oil volume fraction at which $C_{0}^{\prime}$ falls to 1.0

c compressibility factor, $1 / \mathrm{psi}$

C input volume fraction

$C_{o} \quad$ profile parameter

$D \quad$ pipe/well internal diameter, $\mathrm{ft}$

$f_{t p} \quad$ fractional factor

$F_{v} \quad$ velocity sensitivity parameter for liquid-gas flows

$g$ gravitational acceleration, $\mathrm{ft} / \mathrm{sec}^{2}$

$K_{u} \quad$ Kutateladze number

$L \quad$ test section length, $\mathrm{ft}$

$m \quad$ drift velocity multiplier for water-gas flows

$m^{\prime} \quad$ drift velocity multiplier for oil-water flows

$m_{0} \quad$ drift velocity multiplier for vertical water-gas flows

M molecular weight, $\mathrm{lb} / \mathrm{mol}$

$n_{1} \quad$ deviation effect parameter for water-gas flows

$n_{2}$ deviation effect parameter for water-gas flows

$n^{\prime} \quad$ drift velocity exponent for oil-water flows

$n_{1}^{\prime} \quad$ deviation effect parameter for oil-water flows

$n_{2}^{\prime} \quad$ deviation effect parameter for oil-water flows

$n_{3}^{\prime} \quad$ deviation effect parameter for oil-water flows

$N \quad$ number of experimental points

$P \quad$ pressure, $\mathrm{psi}$

$q \quad$ volume flow rate of inflow or outflow, $\mathrm{ft}^{3} / \mathrm{sec}$

$Q \quad$ volumetric flow rate, $\mathrm{ft}^{3} / \mathrm{sec}$

$R \quad$ gas constant 


$\begin{array}{ll}R e & \begin{array}{l}\text { Reynolds number } \\ t\end{array} \\ T & \text { time, } \mathrm{sec} \\ V & \text { temperature, }{ }^{\circ} \mathrm{F} \\ V_{c} & \text { velocity, } \mathrm{ft} / \mathrm{s} \\ V_{d} & \text { characteristic velocity, } \mathrm{ft} / \mathrm{sec} \\ V_{d}^{\prime} & \text { oil-water drift velocity, } \mathrm{ft} / \mathrm{sec} \\ V_{s} & \text { superficial gas velocity, } \mathrm{ft} / \mathrm{sec} \\ x & \text { position along the wellbore, } \mathrm{ft} \\ X_{P} & \text { vector of parameters optimized }\end{array}$

\section{Greek}

$\alpha \quad$ in situ volume fraction or holdup

$\varepsilon \quad$ pipe roughness

$\mu \quad$ viscosity, $\mathrm{cp}$

$\theta \quad$ well deviation from vertical, deg

$\rho \quad$ density, $\mathrm{lb}_{\mathrm{m}} / \mathrm{ft}^{3}$

$\sigma \quad$ interfacial tension/surface tension, $\mathrm{lb} / \mathrm{ft}$

\section{Subscripts}

$\begin{array}{ll}a & \text { accelerational } \\ f & \text { fractional } \\ g & \text { gas } \\ g l & \text { gas - liquid } \\ g o & \text { gas - oil } \\ g w & \text { gas - water } \\ h & \text { hydrostatic } \\ i & \text { segment number } \\ i m & \text { inflow mixture } \\ m & \text { mixture } \\ o & \text { oil }\end{array}$




$\begin{array}{ll}\text { ow } & \text { oil - water } \\ p & \text { phase } \\ w & \text { water }\end{array}$

\subsection{References}

Abduvayt, P., Manabe, R. and Arihara, N.: "Effects of Pressure and Pipe Diameter on GasLiquid Two-Phase Flow Behavoir in Pipelines", SPE paper 84229 presented at the 2003 SPE Annual Technical Conference and Exhibition, Denver, CO, 5-8 October.

Ansari, A.M., Sylvester, N.D., Sarica, C., Shoham, O. and Brill, J.P.: "A Comprehensive Mechanistic Model for Upward Two-Phase Flow in Wellbores", SPE Prod. \& Fac. (May 1994) 143-151.

Aziz, K., Govier, G.W. and Fogarasi, M.: "Pressure Drop in Wells Producing Oil and Gas", J. Cdn. Pet. Tech. (July-Sep. 1972) 38-48.

Danielson, T.J., Brown, L.D. and Bansal, K.M.: "Flow Management: Steady-State and Transient Multiphase Pipeline Simulation", OTC paper 11965 presented at the 2000 Offshore Technology Conference, Houston, TX, 1-4 May.

Díaz, R.L.: "Parameter Determination for Simplified Models of Two and Three-Phase Flow in Wells", MS Thesis, Stanford University, 2004.

Fairhurst, C.P. and Rarrett, N.: "Oil/Water/Gas Transport in Undulating Pipelines - Field Observations, Experimental Data, and Hydraulic Model Comparisons”, SPE paper 38811 presented at the 1997 SPE ATCE, San Antonio, TX, 5-8 October.

Flores, J.G., Sarica, C., Chen, T.X. and Brill, J.P.: "Investigation of Holdup and Pressure Drop Behavior for Oil-Water Flow in Vertical and Deviated Wells", JERT, Trans. ASME (1998) 120, 8-14.

Franca, F. and Lahey Jr, R.T.: "The Use of Drift-Flux Techniques for the Analysis of Horizontal Two-Phase Flows", Int. J. Multiphase Flow (1992) 18, 887-901.

Haaland, S.E.: "Simple and Explicit Formula for the Friction Factor in Turbulent Pipe Flow Including Natural Gas Pipelines," IFAG B-131, Technical Report, Division of Aero- and Gas Dynamics, The Norwegian Institute of Technology, Norway, 1981.

Harmathy, T.Z.: "Velocity of Large Drops and Bubbles in Media of Restricted Extent", AIChEJ (1960) 6, 281-290.

Hasan, A.R. and Kabir, C.S.: "A Study of Multiphase Flow Behavior in Vertical Wells", SPE Prod. Eng. (May 1988a), 263-272.

Hasan A.R. and Kabir C.S.: "Predicting Multiphase Flow Behavior in a Deviated Well," SPE Prod. Eng. (Nov. 1988b) 474-482. 
Hasan, A.R., Kabir, C.S. and Srinivasan, S.: "Void Fraction Estimation in Countercurrent Vertical Two-phase Flow", Chem. Eng. Sci. (1994) 49, 2567-2574.

Hasan, A.R. and Kabir, C.S.: "A Simplified Model for Oil/Water Flow in Vertical and Deviated Wellbores”, SPE Prod. \& Fac. (February 1999) 56-62.

Hill, A.D.: “A Comparison of Oil-Water Slip Velocity Models Used for Production Log Interpretation", J. Pet. Sci.\& Eng. (1992) 8, 181-189.

Hill, A.D.: "Reply", J. Pet. Sci.\& Eng. (1993) 10, 61-65.

Holmes, J.A.: "Description of the Drift Flux Model in the LOCA Code RELAP-UK", I. Mech. E. paper 206/77, Proceedings of the Conference on Heat and Fluid Flow in Water Reactor Safety, Manchester, UK, September 1977.

Holmes, J.A., Barkve, T. and Lund, O.: "Application of a Multisegment Well Model to Simulate Flow in Advanced Wells", SPE paper 50646 presented at the 1998 European Petroleum Conference, The Hague, October 20-22.

Holmes, J.A.: "Well Modeling and Optimization", Proceedings of the $7^{\text {th }}$ International Forum on Reservoir Simulation, Bühl/Baden-Baden, Germany, June 23-27, 2003.

Ishii M.: "One-Dimensional Drift-Flux Model and Constitutive Equations for Relative Motion Between Phases in Various Two-Phase Flow Regimes", Argonne National Lab Report, (October 1977) ANL 77-47.

Jepson, W.P. and Taylor, R.E.: "Slug Flow and Its Transitions in Large-Diameter Horizontal Pipes", Int. J. Multiphase Flow (1993) 19, 411-420.

Nassos G. P. and Bankoff S. G.: "Slip Velocity Ratios in Air-Water System Under Steady State and Transient Conditions", Chem. Eng. Sci. (1967) 22, 661-668.

Oddie, G., Shi, H., Durlofsky, L.J., Aziz, K., Pfeffer, B. and Holmes, J.A.: "Experimental Study of Two and Three Phase Flows in Large Diameter Inclined Pipes", Int. J. Multiphase Flow (2003) 29, 527-558.

Ouyang, L-B.: "Single Phase and Multiphase Fluid Flow in Horizontal Wells", PhD Dissertation, Stanford University, 1998.

Ozon, P.M., Ferschneider, G., and Chwetzoff, A.: "A New Multiphase Flow Model Predicts Pressure and Temperature Profiles in Wells", SPE paper 16535 presented at the 1987 Offshore Europe Conference, Aberdeen, UK, 8-11 September.

Petalas, N. and Aziz, K.: "A Mechanistic Model for Multiphase Flow in Pipes", J. Can. Pet. Tech. (2000) 39, 43-55.

Schlumberger GeoQuest, ECLIPSE Technical Description Manual, 2001.

Shi, H., Holmes, J.A., Durlofsky, L.J., Aziz, K., Diaz, L.R., Alkaya, B. and Oddie, G.: "Drift-Flux Modeling of Multiphase Flow in Wellbores", SPE paper 84228 presented at the 2003 SPE Annual Technical Conference and Exhibition, Denver, CO, 5-8 October. 
Shi, H., Holmes, J.A., Durlofsky, L.J., Aziz, K., Diaz, L.R., Alkaya, B. and Oddie, G.: "Drift-Flux Modeling of Multiphase Flow in Wellbores", SPE paper 89836, to be presented at the 2004 SPE Annual Technical Conference and Exhibition, Houston, TX, 26-29 September.

Taitel, Y., Barnea, D. and Brill, J. P.: "Stratified Three Phase Flow in Pipes", Int. J. Multiphase Flow (1995) 21, 53-60.

The MathWorks Inc., MATLAB, 2002.

Wallis, G.B. and Makkenchery, S.: "The Hanging Film Phenomenon in Vertical Annular Two-Phase Flow", Trans. ASME, Series I (1974) 96, 297.

Wallis, G.B.: One-Dimensional Two-Phase Flow, McGraw-Hill, 1969.

Zuber, N. and Findlay, J.A.: "Average Volumetric Concentration in Two-Phase Flow Systems”, J. Heat Transfer, Trans. ASME (1965) 87, 453-468. 


\section{Part III. Novel Approaches to Account for Heterogeneities and the Optimal Deployment of Nonconventional Wells}

Reservoir heterogeneity can significantly affect the performance of nonconventional wells. This was demonstrated in many of the examples in Chapter 4 within the context of semianalytical modeling. In Chapter 6 we develop procedures for accounting for subgrid heterogeneity within a finite difference context. This includes the upscaling of well index and near-well transmissibilities (i.e., the upscaling of single-phase flow parameters) and relative permeability upscaling. We also present an approach for near-well (single-phase) upscaling applicable for radial (or nearly radial) grids, as might be used in the vicinity of the well in multiblock models. This work includes contributions from several MS and PhD students (Oscar Mascarenhas, Christian Wolfsteiner, Mun Hong Hui). Much of the material presented in Chapter 6 was presented in earlier Annual Reports to DOE (in Years 1 and 3) and also in papers (e.g., Mascarenhas and Durlofsky, 2000; Wolfsteiner and Durlofsky, 2002). The radial upscaling and multiblock simulation results reported here were presented earlier in Lee et al. (2003). The two-phase near-well upscaling has not been presented previously (this work is part of Mun Hong Hui's ongoing PhD research).

Chapter 7 addresses the optimum deployment of nonconventional wells. This includes a genetic algorithm for the optimization of well type and location and a conjugate gradient based procedure for optimizing the operation of smart wells. Some of this work was described in earlier Annual Reports to DOE (in Years 3 and 4) and also in papers (e.g., Yeten et al., 2003, 2004) as well as in Burak Yeten's PhD thesis (2003) and Inegbenose Aitokhuehi's MS thesis (2004). A post-doctoral researcher, Vincent Artus, is currently involved in this work.

\section{Accurate Coarse Scale Simulation of Nonconventional Wells in Heterogeneous Reservoirs}

The accurate finite difference modeling of flow in horizontal or other nonconventional wells poses several challenges. One important aspect of this problem is the accurate representation of fine scale reservoir heterogeneity in the near-well region in flow models. This issue arises because the underlying geocellular model typically contains far more detail than can be accommodated in conventional reservoir simulation models. The disparity between the geological model 
(which may include $O\left(10^{7}-10^{8}\right)$ cells) and the simulation model (which is typically $O\left(10^{5}-10^{6}\right.$ ) cells) requires the use of coarsening (or upscaling) techniques.

The objective of this work is to develop and apply upscaling techniques appropriate for the generation of coarse scale simulation models of nonconventional wells. This will allow for the accurate coarse scale simulation of well performance in heterogeneous reservoirs. To accomplish this upscaling, an appropriate local (i.e., limited domain) flow problem and boundary conditions are clearly defined. The solution of this local fine grid problem, coupled with an appropriate post-processing (averaging) of the fine grid results, provides coarse scale equivalent grid block parameters for use in the global, coarse scale simulation model. The accuracy of the coarse scale description can be further improved by 'optimizing' the coarse grid parameters. This is accomplished by applying an optimization procedure to minimize the difference between the local fine scale solution and the solution of the analogous coarse grid problem.

The well index and transmissibility upscaling presented here complements earlier studies directed toward the calculation of equivalent grid block permeability (or upscaled transmissibility) for heterogeneous systems in regions away from flowing wells (see e.g., Durlofsky, 1991; Pickup et al., 1994 and reviews by Wen and Gomez-Hernandez, 1996; Renard and de Marsily, 1997; Durlofsky, 2003). The two-phase upscaling procedure generalizes existing pseudo-relative permeability approaches (see, e.g., Barker and Dupouy, 1999; Darman et al., 2002) for application in the near-well region. Aziz et al. (1999) demonstrated the inaccuracies that can be observed when standard single-phase coarsening techniques (i.e., no specialized upscaling in the near-well region) are applied to horizontal well problems. Specifically, they observed significant differences in primary production results between fine and coarsened simulation models of three-phase flow in complex, heterogeneous reservoirs. Their work demonstrates that the use of standard upscaling procedures can be inappropriate for problems involving horizontal wells.

Previous papers addressing single-phase upscaling in the region near vertical wells (Ding, 1995; Durlofsky et al., 2000) and recent work by Muggeridge et al. (2002) on upscaling with horizontal or deviated wells, are also relevant to this study. In all of these papers, coarse grid flow models in the near-well region were developed through solution of well-driven flow problems on the fine scale and the subsequent calculation of well indexes and modified well-block 
transmissibilities. The details of the methods differ, though they all display significant improvement over standard techniques.

The well index and transmissibility upscaling presented here differs somewhat from the approaches of Ding (1995) and Ding and Urgelli (1997). Here, specific local flow problems involving a single well are defined and solved to determine the coarse grid near-well parameters. In Ding's work, by contrast, the specific local problem (or set of such problems) to be solved is not explicitly specified. In practice, the local problem would likely include the well in question and some number of neighboring wells (Lemouzy, 1997). In this case, the method presented here specifies a smaller local problem in general, which renders the method more efficient computationally.

The local problems solved here, and the subsequent optimization, differ from the approach taken by Durlofsky et al. (2000), who considered only vertical wells and could therefore solve more limited local problems without parameter optimization. In fact, none of the earlier approaches force the detailed agreement between the local fine and coarse grid solutions as is done in the current method. Muggeridge et al. (2002) consider the solution of global fine grid problems as well as the solution of local flow problems for the calculation of the near-well parameters. Their local solution approach shares some similarities with the methodology presented here. Near-well upscaling on radial (or near-radial) grids within the context of multiblock simulation does not appear to have been considered previously. Our procedure is shown to provide an accurate representation of subgrid heterogeneity for this problem.

This chapter proceeds as follows. In section 6.1, well index and transmissibility upscaling in the vicinity of nonconventional wells is presented. The solution of the local fine grid problem and the post-processing and optimization to obtain coarse scale parameters are described. In section 6.2, results from coarse scale simulations of horizontal well performance, for both single and three-phase flow problems, are compared to the fine scale flow results. Next, in section 6.3, we present the two-phase upscaling procedure. Results with this approach appear in section 6.4. Upscaling to radial grids is addressed in section 6.5. The near-well upscaling procedures are shown to improve standard coarse scale models in all cases considered. In some cases, this improvement is quite substantial. 


\subsection{Well Index and Near-Well Transmissibility Upscaling}

The approach for upscaling single-phase near-well parameters is presented in this section. The starting point is a finely gridded, highly detailed geological model. The intent is to generate a coarsened model suitable for flow simulation that maintains the geologic realism of the fine grid model to the extent possible. The approach presented here is also applicable to general nonconventional (e.g., multilateral) wells assuming a fine scale well index is provided. This well index should account for the well geometry and the local reservoir heterogeneity. The procedure for the calculation of such a $W I^{*}$ presented in Chapter 4 could be modified for this purpose. Here we restrict ourselves to horizontal wells that are aligned with the grid. This allows us to view the fine scale finite difference simulations as reference results.

\subsubsection{Local Fine Grid Flow Problem}

The method is developed through a consideration of single-phase incompressible flow, although the approach will be applicable to more general multiphase problems. The governing fine scale equation is the single phase pressure equation formed by combining Darcy's law with conservation of mass (with all quantities dimensionless):

$$
\nabla \cdot(\mathbf{k} \cdot \nabla p)=-q
$$

Here, $p$ is pressure, $\mathbf{k}$ is the permeability tensor which is typically highly variable in space, and $q$ is the source term. Eq. 6-1 can be solved for pressure once boundary conditions and the source term are specified. From this solution the Darcy velocity $(\mathbf{u})$ is determined:

$$
\mathbf{u}=-\mathbf{k} \cdot \nabla p
$$

Standard upscaling procedures (Durlofsky, 1991; Pickup et al., 1994; Wen and GomezHernandez, 1996; Renard and de Marsily, 1997) retain the form of Eqs. 6-1 and 6-2 on the coarse grid but introduce coarse scale equivalent grid block permeability tensors $\left(\mathbf{k}^{*}\right)$ in place of $\mathbf{k}$. Analogously, coarse scale transmissibility $T^{*}$ can be computed directly (see Durlofsky, 2003 and

Chen et al., 2003 for a detailed discussion of $\mathbf{k}^{*}$ versus $T^{*}$ upscaling). The $\mathbf{k}^{*}$ or $T^{*}$ for each coarse 
block is computed through the solution of a local flow problem over the fine scale region corresponding to the coarse grid block. This problem is usually solved subject to boundary conditions that are essentially linear in pressure (e.g., periodic or constant pressure - no flux boundary conditions). This type of approach is often adequate in regions away from the well, though in some cases global information must be introduced into the upscaling (Chen et al., 2003).

Near flowing wells, particularly when the permeability field is highly variable over small distances, the local pressure field will not be linear. Therefore, the imposition of a linear pressure field could introduce significant inaccuracy in the coarse scale description. In the near-well region, the coarse scale parameters are, therefore, more accurately determined by the solution of a local fine scale problem with flow driven by the actual well. This is the type of fine grid problem that will be solved to generate the coarse scale model in the near-well region.

There is another distinction between the present method and standard approaches for upscaling in regions away from wells. In many upscaling methods, the local problem solved for the calculation of $\mathbf{k}^{*}$ or $T^{*}$ contains only the fine grid blocks corresponding to the target coarse scale region. However, in the near-well upscaling approach presented here (and in other methods developed for this problem), the flow domain is expanded to include neighboring regions around the well. This introduces some additional computation, though the overall upscaling method still represents a very small overhead relative to the ultimate multiphase, time-dependent, global flow simulation. The flow domain used for this solution is referred to as an "extended local" region or as a "local well model."

A cross section (perpendicular to the axis of the horizontal well) of the flow domain for the near-well solution is shown schematically in Fig. 6-1. Finer grid lines correspond to the fine grid and heavier lines to the coarse grid. The domain actually used is fully three dimensional and contains the entire well in addition to surrounding regions. In the figure, the well is completed in the central grid block as shown. The domain included in the local fine grid solution includes the regions corresponding to the coarse grid well block and some number of surrounding coarse grid blocks (in $x, y$ and $z$ ). The fine grid region is referred to as near-well[1], if one 'ring' of coarse grid cells around the well block is included (the shaded region in the figure corresponds to nearwell[1]). When two rings of cells around the well block are included the fine grid region is re- 
ferred to as near-well[2] (the full domain in Fig. 6-1). For a horizontal well completed in five coarse blocks $(5 \times 1 \times 1)$, each of which is comprised of $3 \times 3 \times 3=27$ fine blocks, the local fine grid problem to be solved will contain $7 \times 3 \times 3 \times 27=1701$ cells for near-well[1] and $9 \times 5 \times 5 \times 27=6075$ cells for near-well[2]. Note that, for wells completed in many coarse scale blocks, enhanced efficiency could be obtained by subdividing the local problem into two or more sub-problems. This will result in a reduction in computation but could lead to some degradation in accuracy.

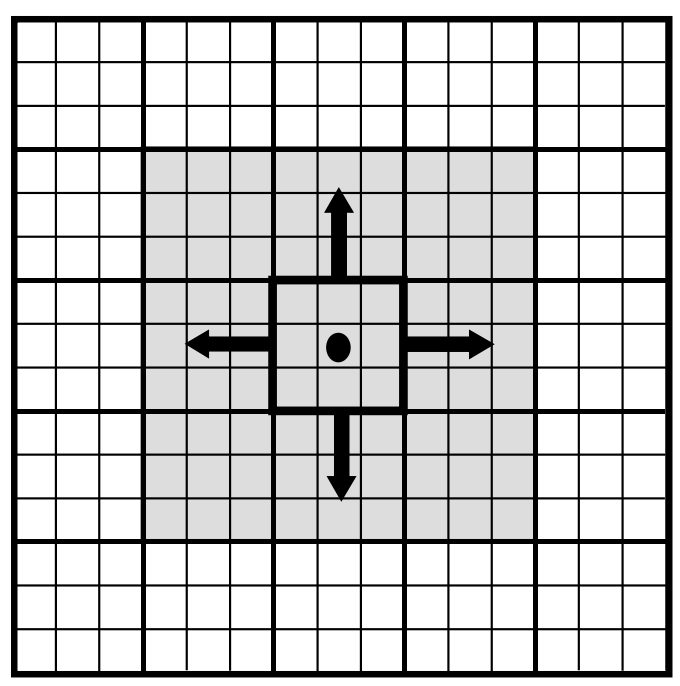

Figure 6-1: Schematic showing cross section of the near-well[1] (shaded) and near-well[2] fine and coarse grid regions (local well model)

Eq. 6-1 is solved over the fine scale region defined above. The wellbore pressure $\left(p_{w b}\right)$ is set to 1 and the pressure on the domain boundaries to zero. This is the source and boundary specification used previously for the vertical well case by Durlofsky et al. (2000). Variation of these boundary conditions was found to have only a small effect on the coarse scale parameters computed from this solution, particularly for near-well[2], in most cases. In some cases, however, the results will be sensitive to these boundary conditions (in which case global flow information can be introduced into the upscaling). The fine grid solution is accomplished using a standard finite difference procedure, with transmissibilities computed using harmonic averaging of the appropriate component of permeability; e.g., for the transmissibility (in the $x$-direction) between cells $i, j, k$ and $(i+1, j, k)$ : 


$$
T_{i+1 / 2, j, k}=\frac{2 k_{i, j, k} k_{i+1, j, k} \Delta y \Delta z}{\left(k_{i, j, k}+k_{i+1, j, k}\right) \Delta x},
$$

where $k$ here refers to the $x$-component of permeability and $\Delta x, \Delta y, \Delta z$ are the grid block sizes in the $x, y$, and $z$-directions. Note that transmissibility relates the flow rate, from block $i, j, k$ to block $i+1, j, k$, to the discrete pressure difference between the cells; i.e.,

$$
q_{i+1 / 2, j, k}=T_{i+1 / 2, j, k}\left(p_{i, j, k}-p_{i+1, j, k}\right),
$$

where $q_{i+1 / 2, j, k}$ is the flow rate between blocks $i, j, k$ and $i+1, j, k$ and $p_{i, j, k}$ and $p_{i+1, j, k}$ are the grid block pressures.

Analogous expressions define $T_{i, j+1 / 2, k}$ and $T_{i, j, k+1 / 2}$. The resulting linear system can be written as $\mathbf{A p}=\mathbf{b}$, where $\mathbf{A}$ is a banded matrix (seven diagonals for the three dimensional problem), $\mathbf{p}$ represents the unknown pressures and $\mathbf{b}$ is the right hand side vector.

The well flow rate, well block pressure and the wellbore pressure for the local fine grid problem are related through the well index WI (as discussed in Chapter 4); i.e.,

$$
q=W I\left(p_{w b}-p_{i, j, k}\right),
$$

where $p_{i, j, k}$ is the well block pressure. In the case of a well flowing at fixed pressure, inserting this representation for $q$ in Eq. 6-1 yields a contribution to $\mathbf{b}$ and a contribution on the main diagonal of $\mathbf{A}$. The well index for the horizontal well (on the fine grid) is computed using a standard procedure (Peaceman, 1983). Following the solution of the local fine grid problem, the flow rates through each of the grid block faces are computed by Eq. 6-4.

\subsubsection{Local Coarse Grid Flow Problem.}

The fine grid solution provides pressures at the grid block centers and the flow rates through each of the well block faces. The grid block pressures are then averaged from this solution over regions corresponding to coarse grid blocks. A bulk volume weighting is used in this averaging process. In addition, the flow rates are summed from the fine grid solution over regions corresponding to the faces of the coarse blocks. The average of the fine grid pressures over a coarse

scale well block is designated $p_{c}$; the average of the fine grid pressures over blocks adjacent to the coarse scale well block is designated as $p_{c, n}$. The quantity $q_{f}$ represents the total flow rate 
through the fine grid region corresponding to the face between coarse blocks $c$ and $c, n$ and $q_{0}$ represents the total flow rate from the well into the coarse scale well block.

The coarse scale quantities to be computed are the well index $\left(W I^{*}\right)$ and the transmissibilities between the well blocks and adjacent coarse grid blocks (referred to as $T_{w}^{*}$ ). From the averaged variables defined above, these quantities can be readily calculated through equations of the form of Eqs. 6-4 and 6-5:

$$
\begin{aligned}
& T_{w}^{*}=q_{f} /\left(p_{c}-p_{c, n}\right), \\
& W I^{*}=\frac{q_{0}}{\left(p_{w b}-p_{c}\right)} .
\end{aligned}
$$

More details on the calculation of $W I^{*}$ and $T_{w}^{*}$, for the case of vertical wells, are provided by Durlofsky et al. (2000).

For the case of a horizontal well aligned along a coordinate direction, the following coarse grid quantities are calculated. For each coarse block in which the well is completed, a $W I^{*}$ (linking the wellbore to the well block) and four or five $T_{w}^{*}$ (linking the well block to all adjacent blocks not containing the well) are computed. For the other connections (which link the well block to adjacent well blocks), the appropriate component of $\mathbf{k}^{*}$ or $T^{*}$ (computed from the local linear flow solution) is used; that is, this transmissibility is not computed from the solution of the well-driven flow problem. In limited tests, the overall coarse scale flow results did not appear to be sensitive to the specific treatment of the well block to well block transmissibility. Note that, in all cases (e.g., even when the local fine grid solution is over the near-well[2] region), only the transmissibilities linking well blocks to adjacent blocks are modified. All other transmissibilities in the coarse model are computed using the $\mathbf{k}^{*}$ or $T^{*}$ computed from the solution of linear flow problems.

The averaging procedure described above provides accurate coarse scale simulation results in many cases. In some cases, however, an improvement in the accuracy of the coarse scale model can be obtained through use of an optimization procedure. The degradation in accuracy observed for the averaging procedure may be due to the high aspect ratios $(\Delta x / \Delta z$ or $\Delta y / \Delta z)$ of the cell faces intersected by the horizontal well, coupled with the high levels of heterogeneity present (high 
variation over small distances in the $z$-direction). This can act to reduce the accuracy of the procedure used to estimate coarse grid pressures from averages of fine grid pressures. Specifically, the $p_{c}$ and $p_{c n}$ in Eqs. 6-6 and 6-7, computed from the averaging procedure, may not always agree closely with the pressures computed via solution of the corresponding coarse grid problem. This in turn can lead to some inaccuracy in the coarse grid quantities of interest, $W I^{*}$ and $T_{w}^{*}$.

To improve the accuracy of the coarse grid model, an optimization procedure was implemented to minimize the difference between the local fine and coarse grid solutions. This is accomplished by optimizing the $W I^{*}$ and $T_{w}^{*}$ such that the differences between the coarse grid flow rates (through each well block face) and the corresponding integrated fine grid flow rates are minimized. These flow rates are indicated by the arrows pointing out of the well block in Fig. 61. The objective function $E$ is defined as:

$$
E=\left(\sum^{n w b s} \sum^{n f a c e s}\left(q_{c}-q_{f}\right)^{2}\right)
$$

where $q_{c}$ designates the coarse grid flow rates, $q_{f}$ the fine grid flow rates (summed over the appropriate region), $n w b s$ designates the number of coarse grid blocks in which the well is completed and nfaces the number of grid block faces in each block through which flow rate is computed (nfaces $=4$ or 5 ). The objective function $E$ is minimized over $\alpha$, where $\alpha$ designates the $W I^{*}$ and $T_{w}^{*}$ in all coarse scale blocks containing the well and $\alpha^{*}$ represents the set of parameters that minimizes Eq. 6-8. The minimum in $E$ will occur when $\nabla E$ (gradient with respect to $\alpha$ ) is driven to zero.

This fully coupled problem is solved by iterating on $\alpha$ using a Gauss Newton optimization algorithm. The Gauss Newton method is a gradient based procedure and derives from a Taylor series expansion of the objective function $E$ about the previous estimate for $\alpha$ (designated $\alpha^{k}$ ). At each iteration, an equation of the form

$$
\mathbf{H}_{G N} \Delta \alpha=-\nabla E
$$

is solved, where $\mathbf{H}_{G N}$ is the Gauss Newton Hessian matrix; i.e., the Hessian matrix modified to guarantee a direction of descent and eliminate the need for calculating second derivatives, and 
$\Delta \alpha=\alpha^{k+1}-\alpha^{k}$. The elements of $\nabla E$ and $\mathbf{H}_{G N}$ are computed numerically using a forward difference approximation. Each row of $\mathbf{H}_{G N}$, and each element of $\nabla E$, requires the solution of the coarse grid near-well problem with one of the $\left(W I^{*}, T_{w}^{*}\right)$ parameters perturbed (for a total of about $5 \times n w b s$ coarse grid solutions at each iteration). In order to minimize the number of iterations, some additional features are introduced into the optimization algorithm (see Mascarenhas, 1999; Mascarenhas and Durlofsky, 2000 for details). The optimum is usually achieved in about 4-6 iterations of the coupled system. This is not overly time consuming because these iterations involve only coarse grid solutions.

\subsection{Numerical Results for Flow Driven by Horizontal Wells}

Detailed flow results are now presented for flow driven by horizontal wells in heterogeneous systems. Single and three phase flow scenarios are considered. In all cases, the fine grid model is a geostatistical realization of prescribed statistics. The geostatistical realizations were generated using GSLIB (Geostatistical Software Library) (Deutsch and Journel, 1998). The flow simulations were performed using the ECLIPSE commercial simulator.

Coarse grid models are generated by uniformly coarsening the fine grid permeability field (i.e., coarsening by a fixed factor in each coordinate direction) and computing equivalent grid block permeabilities $\mathbf{k}^{*}$ for each coarse grid block using standard pressure - no flow boundary conditions. Uniform coarsening is known to be sub-optimal; e.g., Durlofsky et al. (1997) demonstrated significant improvement in coarse scale displacement results when the fine grid is nonuniformly coarsened. This nonuniform coarsening is of particular importance when the flow is strongly impacted by connected regions of high permeability (e.g., layering), as is often the case for displacement processes involving vertical wells in layered systems. For the primary production problems considered below, with flow driven by horizontal wells, reservoir performance may be less dominated by connected regions of high permeability than in the vertical well case. Therefore, nonuniform coarsening is probably less essential and a uniformly coarsened grid may prove adequate. Nonetheless, nonuniform coarsening would still be expected to provide improved accuracy in displacement calculations. 
For the single phase flow cases discussed below, results are presented in terms of well productivity $(q / \Delta p)$ at steady state and flow profiles along the horizontal well (i.e., inflow versus normalized position along the well). In all cases, the fine grid results are compared to the coarse grid results with no near-well upscaling (the standard approach) and to coarse grid results with the near-well upscaling procedure described above. In computing results using the standard approach, $\mathbf{k}^{*}$ is calculated for each grid block (including well blocks) and then the coarse scale well index is computed using the Peaceman (1983) method as applied to the grid block $\mathbf{k}^{*}$. All transmissibilities are computed directly from the $\mathbf{k}^{*}$. In some of the cases discussed below, results are presented using both near-well[1] and near-well[2] upscaling. Unless otherwise stated, the coarse scale models are generated using the optimization procedure described above. For clearer comparisons between fine and coarse grid results, the fine grid inflow profiles are summed over regions corresponding to the coarse grid blocks.

It is important to note that, in this work, the near-well upscaling method is assessed by comparing coarse scale results to the reference fine grid results. For present purposes, therefore, the fine grid results are considered to be "correct." However, the fine grid model itself may not always provide numerical results that are converged (or nearly so). Thus, even if the coarsened model matches the fine grid result exactly, it may still reflect the error inherent in the fine grid solution. This potential source of error can be minimized through the use of higher levels of resolution in the fine grid model.

\subsubsection{Single Phase Flow Results}

In the first example, the permeability field is log-normally distributed, with the logarithm of permeability specified to be of a prescribed variance $\left(\sigma^{2}\right)$ and correlation structure. The correlation structure is specified in terms of the dimensionless correlation lengths $l_{x}, l_{y}$ and $l_{z}$, where each correlation length is nondimensionalized by the system length in the corresponding direction. A single horizontal well of length $2100 \mathrm{ft}$ centered in a formation of physical dimensions $5100 \mathrm{ft} \times$ $5100 \mathrm{ft} \times 225 \mathrm{ft}$ is considered. The total production rate is set to $50,000 \mathrm{bbl} / \mathrm{d}$. The top and bottom of the system are maintained at a fixed pressure (the average reservoir pressure is about 3000 psi) and there is no flow through the sides. These boundary conditions are meant to qualitatively represent a reservoir underlain by a large aquifer (and overlain by a large gas cap) that act to ap- 
proximately maintain pressure above and below the oil region. After a brief transient period, the system achieves a steady state and the wellbore pressure reaches a constant value.

The fine scale model is of dimension $(\mathrm{NX} \times \mathrm{NY} \times \mathrm{NZ}$ ) $51 \times 51 \times 45$ (a total of 117,045 cells) and the coarse grid (coarsened by a factor of 3 in each direction) is of dimension $17 \times 17 \times 15$ (4335 cells). We set $l_{x}=l_{y}=0.5, l_{z}=0.05$ and $\sigma=2$. This type of permeability field corresponds to an incompletely layered system. The inflow profiles for this case are shown in Fig. 6-2. Results using standard coarsening for this more highly heterogeneous case are in considerable error relative to the fine grid results, while those using the near-well upscaling are in very close agreement. The productivity results (Fig. 6-3) show that standard coarsening gives an error of about 32\%; near-well[2] upscaling reduces this error to about 3\%. This example illustrates that, in some cases, near-well upscaling is necessary both to capture the correct inflow profile and to provide the correct wellbore pressure for a prescribed flow rate. The near-well upscaling results with averaging (rather than optimization) are very close (within a few percent) to the results presented here, so the optimization procedure is not essential for these examples.

The results in Figs. 6-2 and 6-3 are typical of many of the other results obtained for single horizontal wells in log-normal permeability fields (see Mascarenhas and Durlofsky, 2000, for another example). Specifically, the standard coarse grid results show considerable errors in the inflow profile, while the results using near-well upscaling show close agreement between the fine and coarse models. Results for well productivity using near-well[1] upscaling show improvement over the coarse grid results; near-well[2] upscaling results in turn display improvement over the near-well[1] results.

The next example is more demanding and involves a single horizontal well in a sand-shale permeability fields. The reservoir is now of dimensions $10,000 \mathrm{ft} \times 5000 \mathrm{ft} \times 100 \mathrm{ft}$ and the well (centered in the reservoir) is of length $2000 \mathrm{ft}$. The permeability descriptions used here are realizations previously generated by Aziz et al. (1999). This field, described in detail by Aziz et al. (1999), represents a fluvial sandstone reservoir with a net to gross ratio of $70 \%$. Sequential indicator simulation was applied to generate the lithofacies model. Shales typically extend over a range of about $100 \mathrm{ft} \times 100 \mathrm{ft} \times 10 \mathrm{ft}$ and are of permeability $1 \mathrm{mD}$. In the sandstone, porosity and permeability are correlated over $O(2000 \mathrm{ft})$ areally and $O(1-10 \mathrm{ft})$ vertically. Sandstone per- 
meability is centered at about $500 \mathrm{mD}$. As discussed above, Aziz et al. (1999) observed significant inaccuracy in coarse scale models generated from these permeability fields.

The fine scale model is of dimension $100 \times 50 \times 32(160,000$ cells $)$ and the coarse grid is of dimension $20 \times 10 \times 12(2400$ cells $)$. The coarse grid is generated from the fine grid via a uniform coarsening (by a factor of five in $x$, five in $y$ and three in $z$, with additional layers at the top and bottom of the model to provide pressure support). As in the previous examples, well flow rates are specified and the simulations are run until steady state is achieved. In this case, we compare the optimization procedure with the simpler averaging approach. In both cases, the near-well[2] region is used in the upscaling procedure.

Production profiles for the four cases are shown in Fig. 6-4. The standard coarse grid results are in considerable error relative to the fine grid results. The near-well[2] scale up results, using either averaging or optimization, are in relatively good agreement with the fine grid results, though the agreement here is not of the level achieved in the log-normal permeability cases. Interestingly, the production peak is captured slightly more accurately using averaging than it is using optimization. The normalized well productivity results are shown in Fig. 6-5. The standard coarse grid model significantly overpredicts the well productivity; the error in $(q / \Delta p)_{\mathrm{n}}$ is about $85 \%$. Results using either of the near-well upscaling methods are in closer agreement with the fine grid model. The near-well[2] with optimization results (NW(o) in the figure) are, in this case, more accurate than the near-well[2] with averaging results (NW(a) in the figure). Specifically, the error in $(q / \Delta p)_{\mathrm{n}}$ is reduced from $32 \%$ (with averaging) to $18 \%$ (with optimization). Qualitatively similar results for another sand/shale realization of the same statistics are shown in Mascarenhas and Durlofsky (2000). 


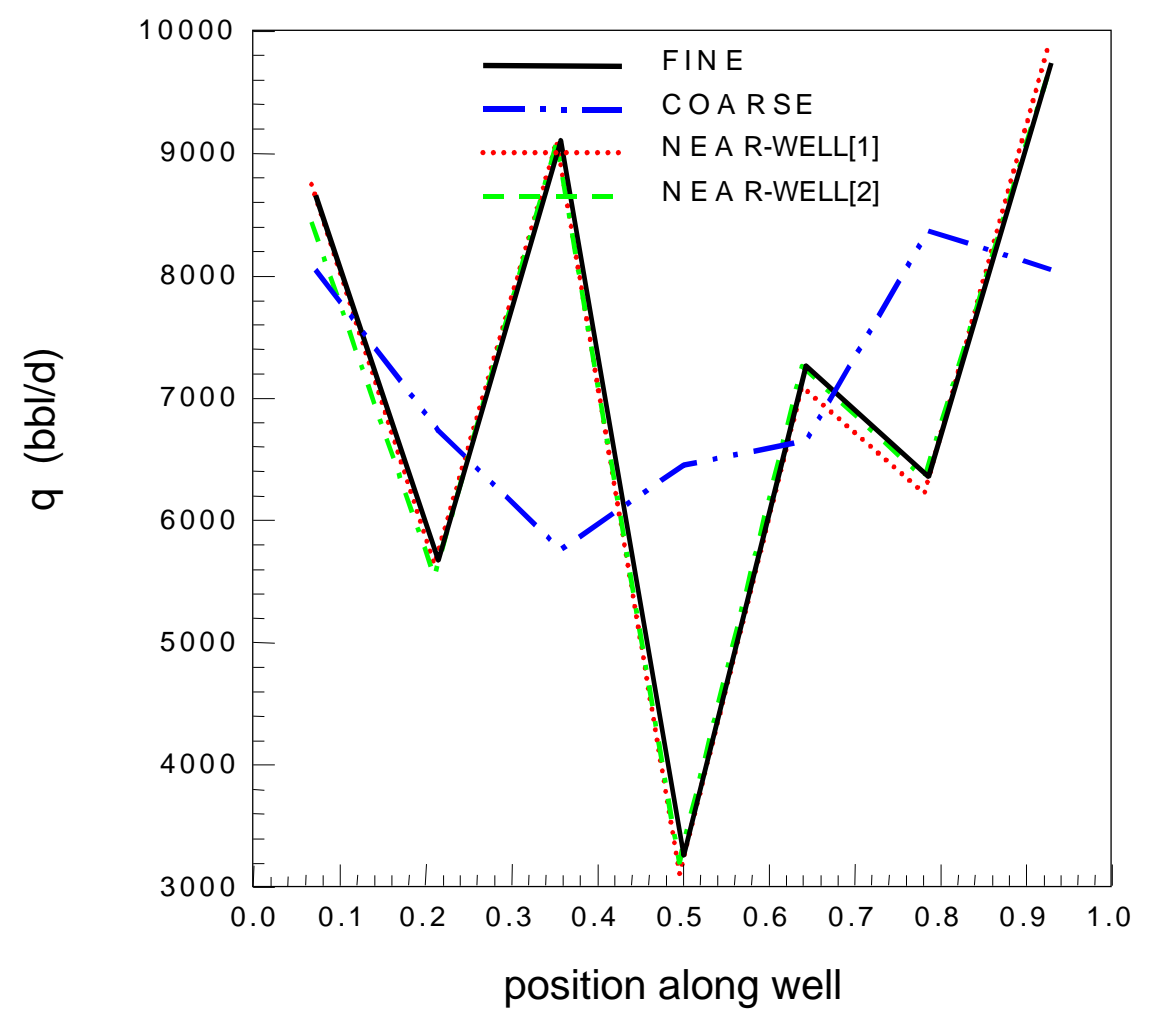

Figure 6.2: Inflow profiles along the well for $l_{x}=l_{y}=0.5, l_{z}=0.05, \sigma=2$

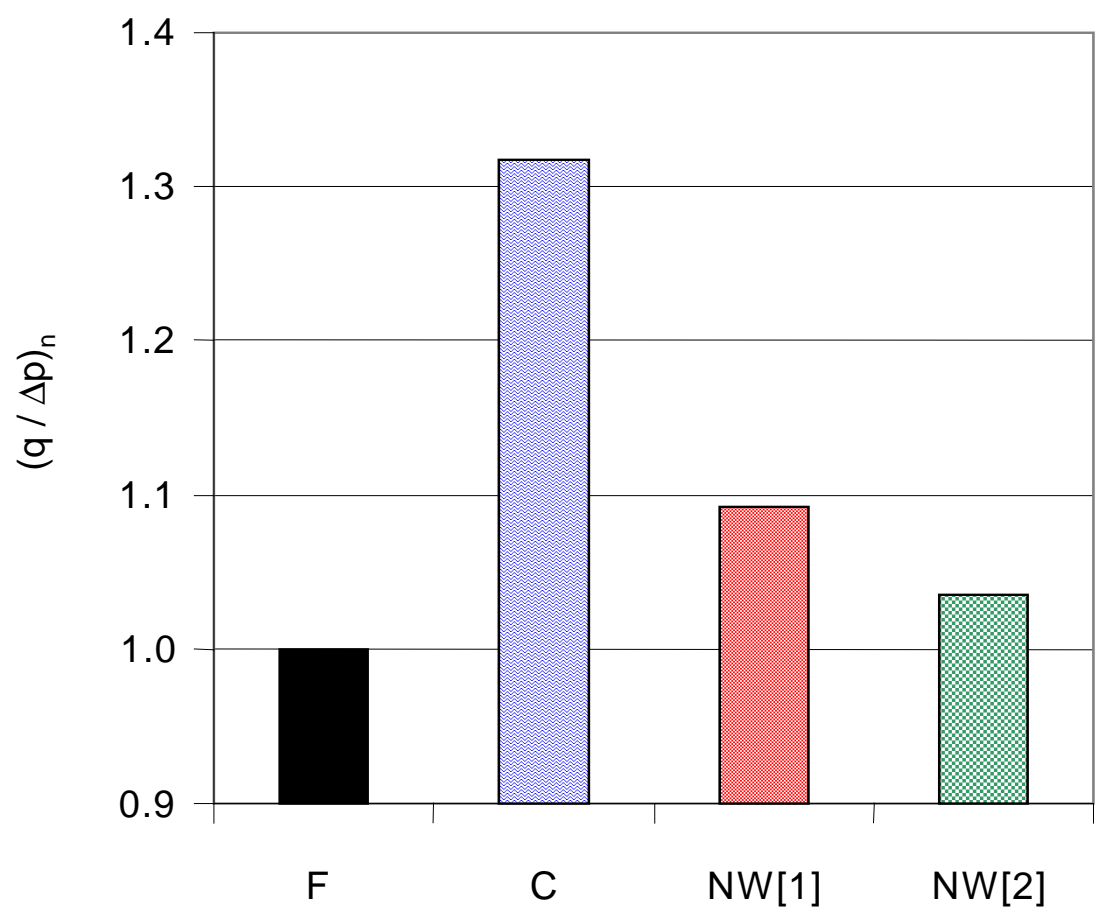

Figure 6.3: Normalized well productivity for $l_{x}=l_{y}=0.5, l_{z}=0.05, \sigma=2$ 


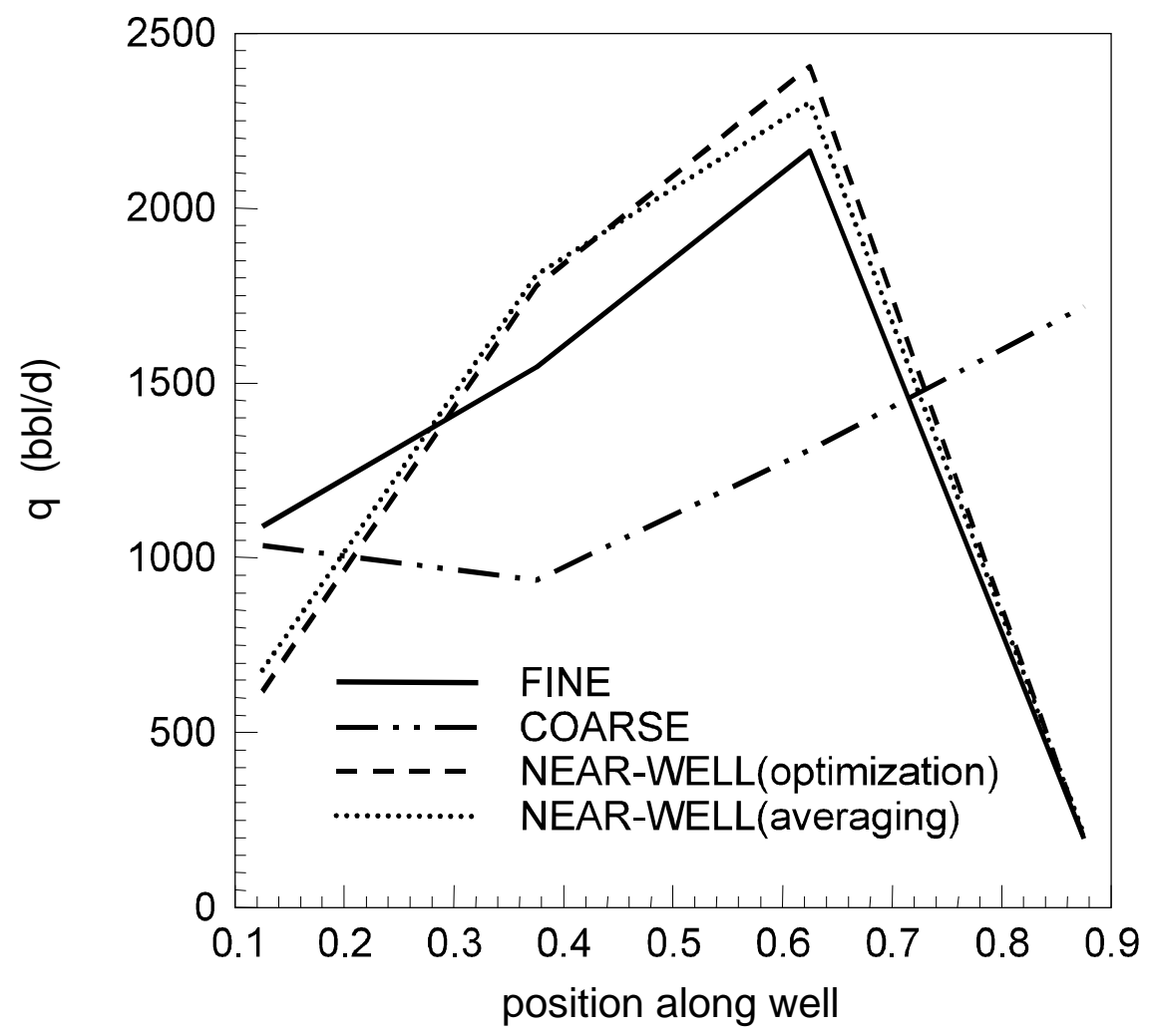

Figure 6-4: Inflow profiles along the well for sand/shale system

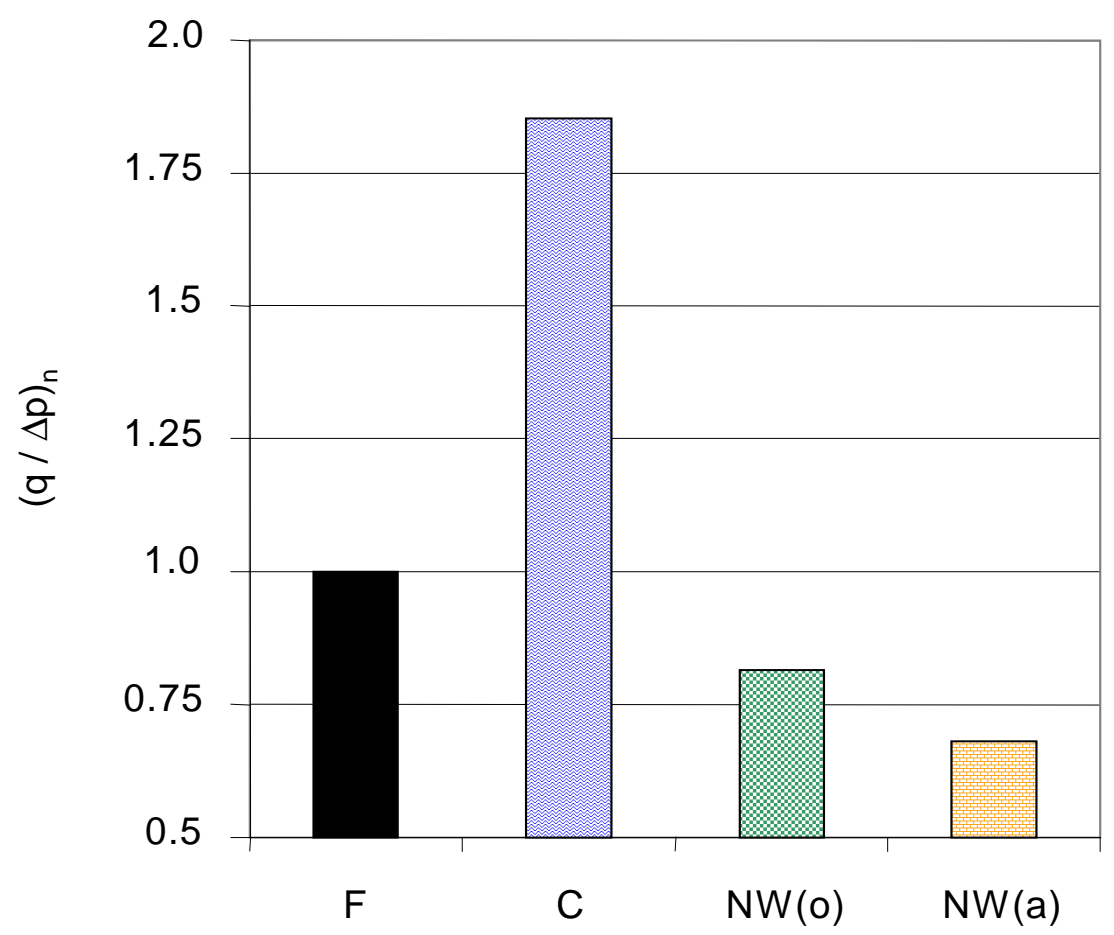

Figure 6-5: Normalized well productivity for sand/shale system 


\subsubsection{Three-Phase Flow Results}

The next case involves a single horizontal well in the oil zone of a sand/shale reservoir containing a large aquifer and a large gas cap. This case was previously considered by Aziz et al. (1999), who showed that standard upscaling techniques could lead to significant error in simulation predictions. The reservoir description here is the same as that in the sand/shale case considered above (Figs. 6-4 and 6-5).

The well is prescribed to produce at $5000 \mathrm{bbl} / \mathrm{d}$, with a minimum bottom hole pressure constraint of 1500 psi. The reservoir boundaries are all specified to be no-flow (refer to Aziz et al., 1999, for a more detailed description of the reservoir and fluid properties). The fine scale model is of dimension $100 \times 50 \times 32(160,000$ cells $)$ and the coarse grid is of dimension $25 \times 25 \times 12$ (7500 cells). The coarse grid is generated from the fine grid via a uniform coarsening (by a factor of four in $x$, two in $y$ and three in $z$, with an additional layer in both the aquifer and the gas cap).

Simulation results for oil production rate and wellbore pressure, for the fine model, standard coarse model, and coarse model with near-well[2] upscaling (with optimization), are shown in Figs. 6-6 and 6-7. The well in the fine grid simulation model reaches the minimum bottom hole pressure constraint relatively early in the run (after about one year) and the well produces at this pressure for the remainder of the simulation. Production clearly declines once the well reaches the minimum bottom hole pressure. The standard coarse model is in considerable error, continuing to produce at the initial rate for about 3000 days (a factor of nearly ten too long). The coarse model with near-well upscaling, by contrast, is in reasonably close agreement with the fine scale result, though the differences are clearly greater than in the single phase flow examples presented above. Nonetheless, the coarse model with near-well upscaling provides results that are substantially better than those obtained using the standard coarse grid model. Results for water cut and GOR are shown in Mascarenhas and Durlofsky (2000). Both results are significantly improved using near-well upscaling (relative to the standard approach), though there is still some error in GOR. It is possible that the error in GOR in the coarse model with near-well upscaling is due to other aspects of the upscaled model, not related to the near-well treatment. 


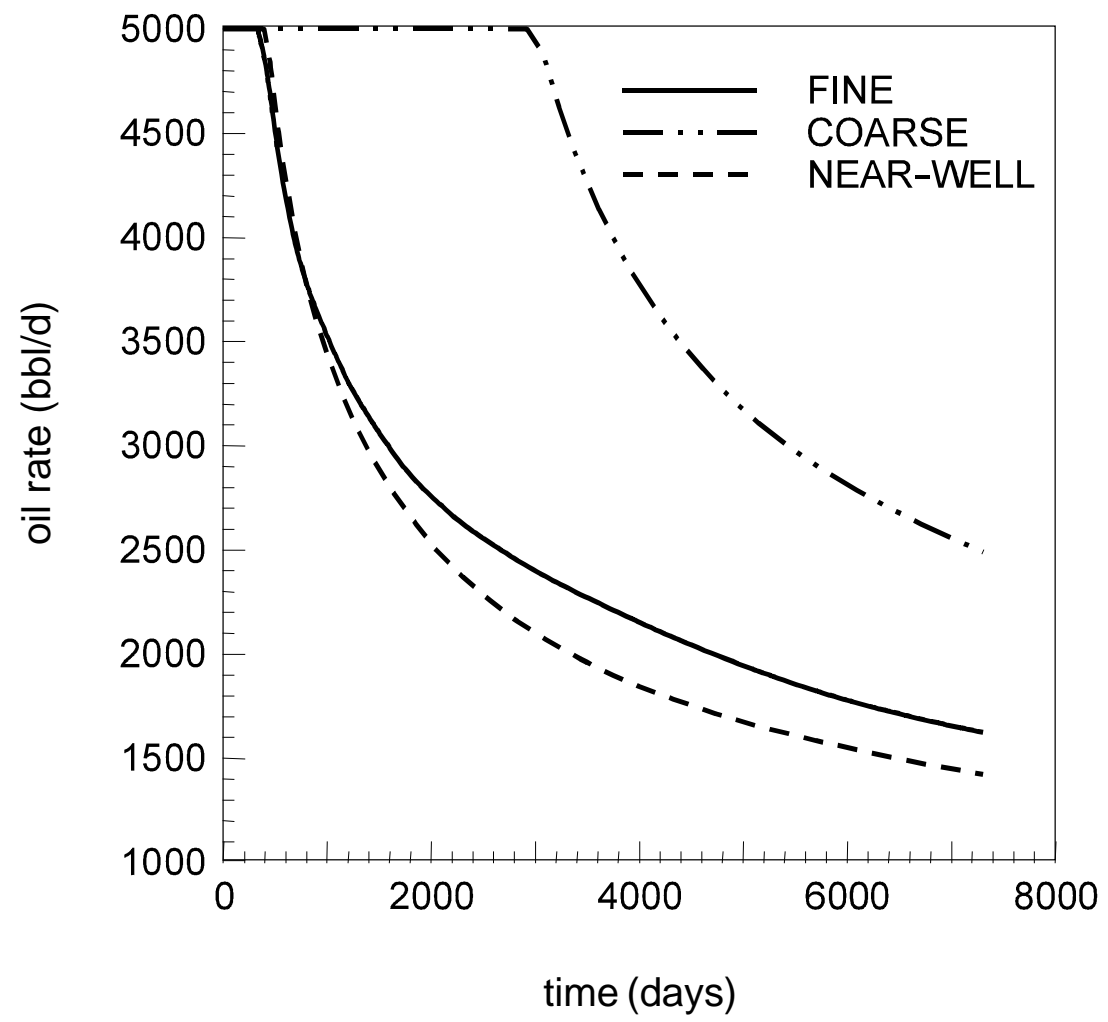

Figure 6-6: Oil production rate (three phase flow) for sand/shale system

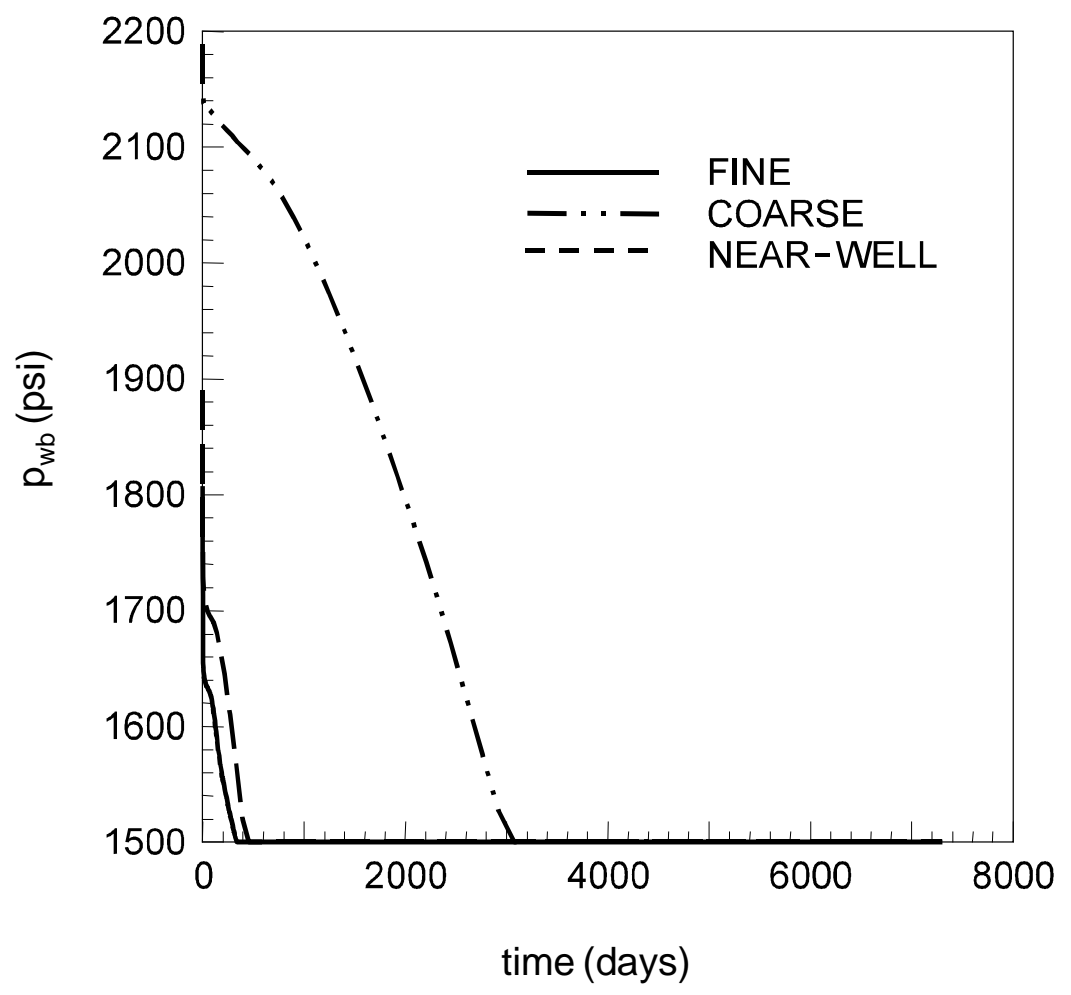

Figure 6-7: Wellbore pressure for sand/shale system 


\subsection{Upscaling of Two-Phase Flow Parameters}

Our two-phase upscaling procedure represents an extension of the near-well, single-phase upscaling method described above. We thus perform our upscaling in the context of the local well model; i.e., the near-well region in which the flow is dominated by the well (Fig. 6-1). Although the near-well, single-phase upscaling technique has been demonstrated to work well in many scenarios, it is less effective in some cases. If multiphase effects are dominant, for instance in an unfavorable mobility ratio oil-water displacement, then the fractional flow behavior of the coarse model may be very different from that of the fine scale model. Two-phase upscaling may be necessary in this situation.

A great number of two-phase upscaling approaches have been proposed to date (see, e.g., Barker and Dupouy, 1999; Darman et al., 2002 for reviews) but very few researchers have considered two-phase upscaling in the context of a near-well problem in recent years. Emanuel and Cook (1974) addressed this problem earlier, but this work was performed at a time when singlephase upscaling techniques were much less developed. The linear flow assumption inherent in most two-phase upscaling approaches is likely to be inappropriate near the well, where the flow is radial. We have modified a popular dynamic pseudo relative permeability upscaling technique (Stone, 1991) to account for the radial flow pattern near the well. We achieve this by imposing a well-driven problem to compute the pseudo (upscaled) relative permeabilities $\left(k_{r j}^{*}\right)$. Nevertheless, as we will see below, this modified method still does not produce entirely satisfactory results in the near-well, two-phase problems we have studied. Instead, we propose using an optimization procedure (along the lines used for the single-phase near-well problem) to obtain the optimized $k_{r j}^{*}$ that will reproduce the two-phase flow behavior of the fine scale local well model.

We first define the local well model for each well to be upscaled (as illustrated in Fig. 6-1). We will describe the method as applied to an injector but the treatment for a producer is analogous. The near-well, single-phase upscaling procedure described above is applied first. This pro-

vides the optimized well index $\left(W I^{*}\right)$ for the well and transmissibilities $\left(T_{w i}{ }^{*}\right)$ for each face of the coarse well block. Away from the coarse well block, we obtain the interblock transmissibilities either via a harmonic averaging of upscaled absolute permeabilities $\left(\mathbf{k}^{*}\right)$ or the using a more sophisticated local-global upscaling procedure (Chen et al., 2003). 
In our near-well, two-phase upscaling technique, we also impose a well-driven problem on the fine scale local well model except the displacement is two-phase (oil-water) in nature. The injected water will displace oil toward the boundaries at a constant pressure drop. The fine grid total oil and water flow rates traversing the area that corresponds to each face of the coarse well block are stored as a function of the average water saturation within that block. The equivalent flow problem is next imposed on the coarse scale local well model and the well two-phase relative permeabilities $\left(k_{r j}^{*}\right)$ are optimized so that the oil and water flow rates of the coarse model match the reference values at the corresponding average saturation.

The objective function $E$ in the two-phase optimization is defined as the following sum of squared residuals:

$$
E(\boldsymbol{\theta})=1 / 2 \sum_{i=1}^{n_{\text {cwb }}}\left[\alpha_{T}\left(\sum_{f=1}^{n_{j}} q_{T, i f}^{\text {coarse }}-\sum_{i=1}^{n_{j}} q_{T, i f}^{\text {fine }}\right)^{2}+\alpha_{w}\left(\sum_{i=1}^{n_{j}} q_{w, i f}^{\text {coarse }}-\sum_{i=1}^{n_{j}} q_{w, i f}^{\text {fine }}\right)^{2}\right]
$$

where $n_{c w b}$ is the number of the coarse grid blocks in which the well is completed, $n_{j}$ is the number of faces of the $j^{\text {th }}$ coarse grid well block $q_{T, i f}^{\text {coarse }}, q_{T, i f}^{\text {fine }}, q_{w, i f}^{\text {coarse }}$ and $q_{w, i f}^{\text {fine }}$ are the coarse total, fine total, coarse water and fine water flow rates at the $f^{\text {th }}$ face of the $i^{\text {th }}$ coarse well block while $\alpha_{T}$ and $\alpha_{w}$ are the optimization weights for the total and water flow rate residuals. The parameter space $\boldsymbol{\theta}$ (of size $n_{c w b} \times 2$ ) is given by:

$$
\boldsymbol{\theta}=\left[\begin{array}{llllll}
k_{r o, 1}^{*} & \ldots & k_{r o, n_{c w b}}^{*} & k_{r w, 1}^{*} & \ldots & k_{r w, n_{c w b}}^{*}
\end{array}\right]^{T}
$$

We employ the Levenberg-Marquardt algorithm (Bishop, 1995), to find the set of parameters $\boldsymbol{\theta}^{\text {opt }}$ that minimizes $E$. Our procedure is applicable for any objective function as long as the problem is not overdetermined; i.e., the number of parameters should be at least equal to the number of variables we wish to match. In our case, there are $\left(n_{c w b} \times 2\right)$ flow rates to match and $\left(n_{c w b} \times 2\right)$ optimization parameters. Unlike the single-phase procedure, for which there is a single set of optimized parameters, here we will determine the optimized $k_{r j}^{*}$ as a function of the coarse well block water saturation, i.e., $k_{r o}^{*}\left(\hat{S}_{w}\right)$ and $k_{r w}^{*}\left(\hat{S}_{w}\right)$. As mentioned earlier, the fine scale simulation, involving a water injector in the local well model, will provide the reference flow rates $\left(q_{T, f}^{f i n e}\right.$ and $\left.q_{w, f}^{\text {fine }}\right)$ as a function of the pore-volume weighted well block water saturation, defined 
as:

$$
\hat{S}_{w}=\frac{\sum_{k=1}^{n_{z, c}} \sum_{j=1}^{n_{y, c}} \sum_{i=1}^{n_{x, c}} \phi_{i j k} V_{i j k} S_{w, i j k}}{\sum_{k=1}^{n_{z, c}} \sum_{j=1}^{n_{y, c}} \sum_{i=1}^{n_{x, c}} \phi_{i j k} V_{i j k}}
$$

where $n_{x, c}, n_{y, c}$ and $n_{z, c}$ are the number of fine grid blocks comprising the coarse grid well block in the $x, y$ and $z$ direction respectively while $S_{w, i j k}, V_{i j k}$ and $\phi_{i, j, k}$ are the water saturation, volume and porosity of fine block $(i, j, k)$ respectively. The simulation needs to be performed out to a relatively mature stage of flooding in order to obtain as wide a range of $\hat{S}_{w}$ as possible. During the simulation of the coarse scale local well model, the optimization algorithm is applied at each time step, producing the set of optimized $k_{r j}^{*}$ that will minimize the mismatch between the coarse

and fine grid oil and water flow rates for the entire range of $\hat{S}_{w}$. Although a considerable number of coarse scale simulations are performed during the optimization, this is not demanding computationally because the coarse local well model typically contains relatively few grid blocks and only a few iterations are required at each time step.

\subsection{Results for Two-Phase Upscaling}

We consider two example cases to demonstrate the performance of our near-well, two-phase upscaling method. The first case involves a vertical water injector that is completed at the center of a $27 \times 27 \times 1$ local well model extracted from a highly channelized reservoir (Fig. 6-8). The displacement occurs under an unfavorable ratio oil/water viscosity of 17; hence water will have a strong preference to flow along high permeability streaks. The fine model is upscaled by a factor of 81 , resulting in a coarse local well model of $3 \times 3$.

The second case entails a horizontal water injector completed in a $15 \times 15 \times 15$ synthetic model (Fig. 6-9) with a log mean of 3 and a log variance of 5 and horizontal and vertical dimensionless correlation lengths of 0.5 and 0.1 respectively. The oil/water viscosity ratio is 10 . The fine model is upscaled by a factor of 125 , resulting in a coarse model of $3 \times 3 \times 3$. 

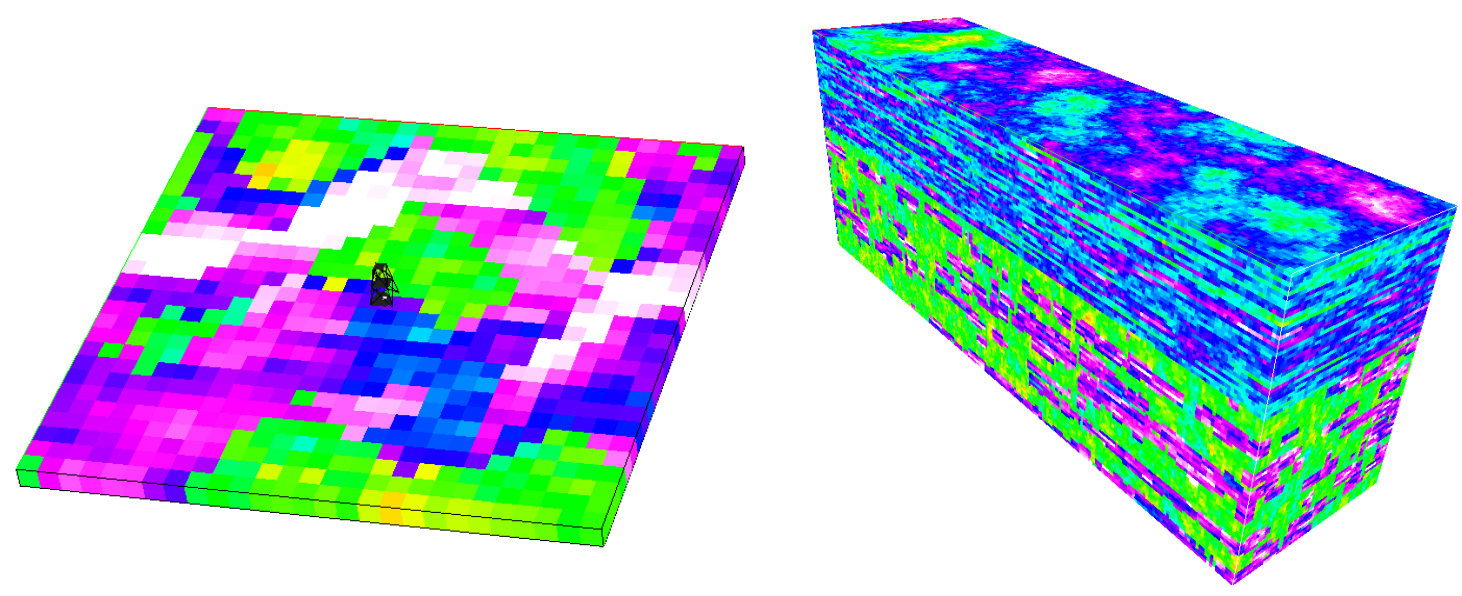

0.0007

$4024.55 \mathrm{mD}$

Figure 6-8: Permeability field of a $27 \times 27 \times 1$ local well model (left) with a vertical water injector; extracted from a channelized reservoir (right)

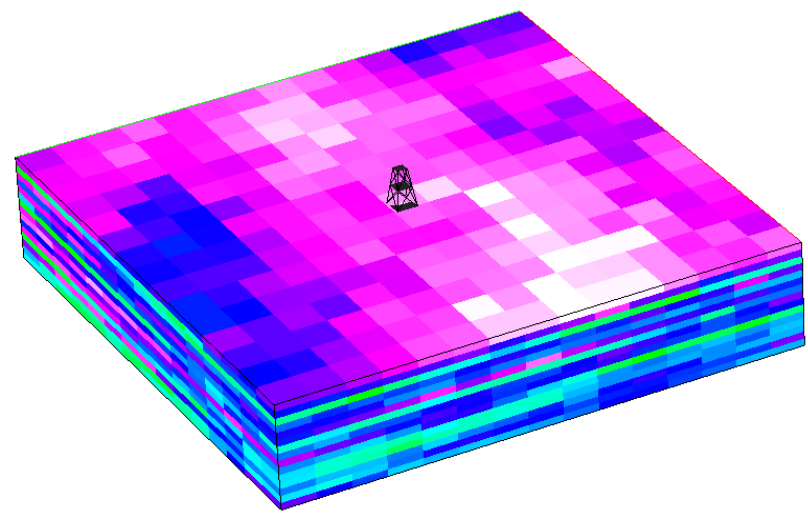

$0.0129 \quad 4130.34 \mathrm{mD}$

Figure 6-9: Permeability field of a $15 \times 15 \times 15$ local well model with a horizontal water injector (well trajectory not visible)

The boundaries of both local well models are set at a constant pressure of 2000 psi while the well bottom hole pressure is specified to be 6000 psi. We consider the oil and water that flow out of the local well model boundaries as the "produced" liquids and plot the oil cut and total flow rate curves as a function of time. We investigate and compare the following upscaling methods: 
1. Our proposed two-phase, near-well upscaling technique

2. Use of standard upscaled absolute permeabilities ( $\mathbf{k}^{*}$ only)

3. Near-well, single-phase upscaling technique

4. Stone's pseudo relative permeabilities applied to the well block (Stone's well $k_{r j}^{*}$ )

We note that the two-phase, near-well upscaling and Stone's well $k_{r j}^{*}$ techniques also include a near-well, single-phase upscaling component; i.e., the optimized $W I^{*}$ and $T_{w i}{ }^{*}$ are employed in conjunction with the $k_{r j}^{*}$.

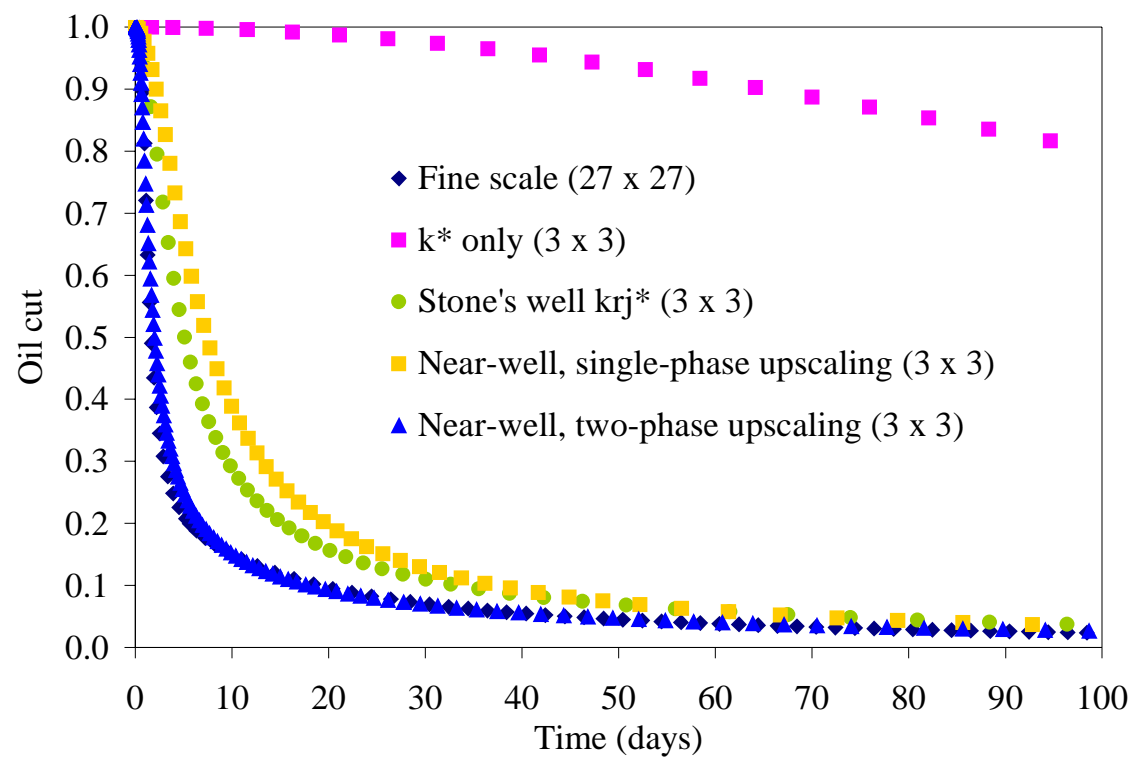

Figure 6-10: Local well model fractional flow curves for a 2D case, comparing the match to the fine scale solution given by different coarse models 


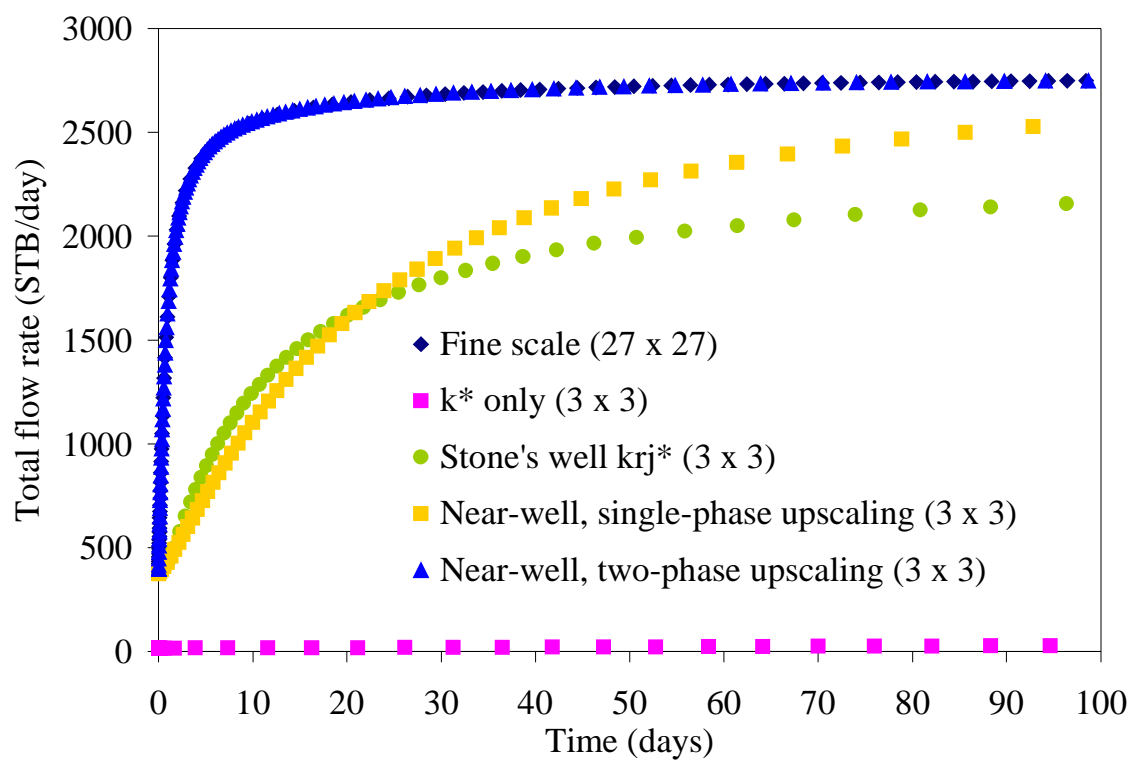

Figure 6-11: Local well model total flow rate curves for a 2D case, comparing the match to the fine scale solution given by different coarse models

From Figs. 6-10 and 6-11, we see that the use of $\mathbf{k}^{*}$ only leads to a gross mismatch of both the oil cut and total flow rate curves compared to the fine scale solutions. In this highly heterogeneous field, near-well effects are very important. In particular, the use of $\mathbf{k}^{*}$ to compute the well index (Peaceman, 1983) will bring about a large inaccuracy in well injectivity. This problem is certainly mitigated by the near-well, single-phase upscaling procedure, which results in a significantly improved match of both curves and yields a match of the fine model steady state flow rate as expected. The use of Stone's well $k_{r j}^{*}$ leads to an oil cut curve that is closer to the fine scale solution compared to that from near-well, single-phase upscaling, although the match of the total flow rate history is worse. Finally, our near-well, two-phase upscaling technique produces an exact match of the fine scale solutions for both curves, indicating that the upscaled local well model is essentially an exact reproduction of the fine scale local well model in terms of fractional flow and total mobility behaviors.

For the 3D case (Figs. 6-12 and 6-13), the trends are similar to those described above for the 2D case. The use of $\mathbf{k}^{*}$ only still leads to the poorest match of both curves compared to the fine scale solutions although the mismatch is somewhat less than that observed in the 2D case, likely because the degree of heterogeneity in this $3 \mathrm{D}$ model is not as extreme. Once again, the near- 
well, single-phase upscaling method brings about a significant improvement of the match of both curves, especially the steady state flow rate. However, in this case, the use of Stone's well $k_{r j}^{*}$ has neither produced any improvement in the match of the oil curve nor any degradation of the match in the total flow rate curve compared to near-well, single-phase upscaling. The errors with singlephase upscaling (either without two-phase upscaling or using Stone's well $k_{r j}^{*}$ ) are small in this case. Even so, our near-well, two-phase upscaling technique provides improvement and reproduces the fine scale solutions very closely.

We conclude that our proposed method is effective in capturing the two-phase flow behavior of the fine scale local well model. The upscaled (pseudo) relative permeabilities determined for the local well model can then be used in the global coarse scale simulation model. In cases for which fine scale near-well effects are important, the use of these near-well pseudo relative permeabilities can be expected to lead to more accurate coarse scale predictions.

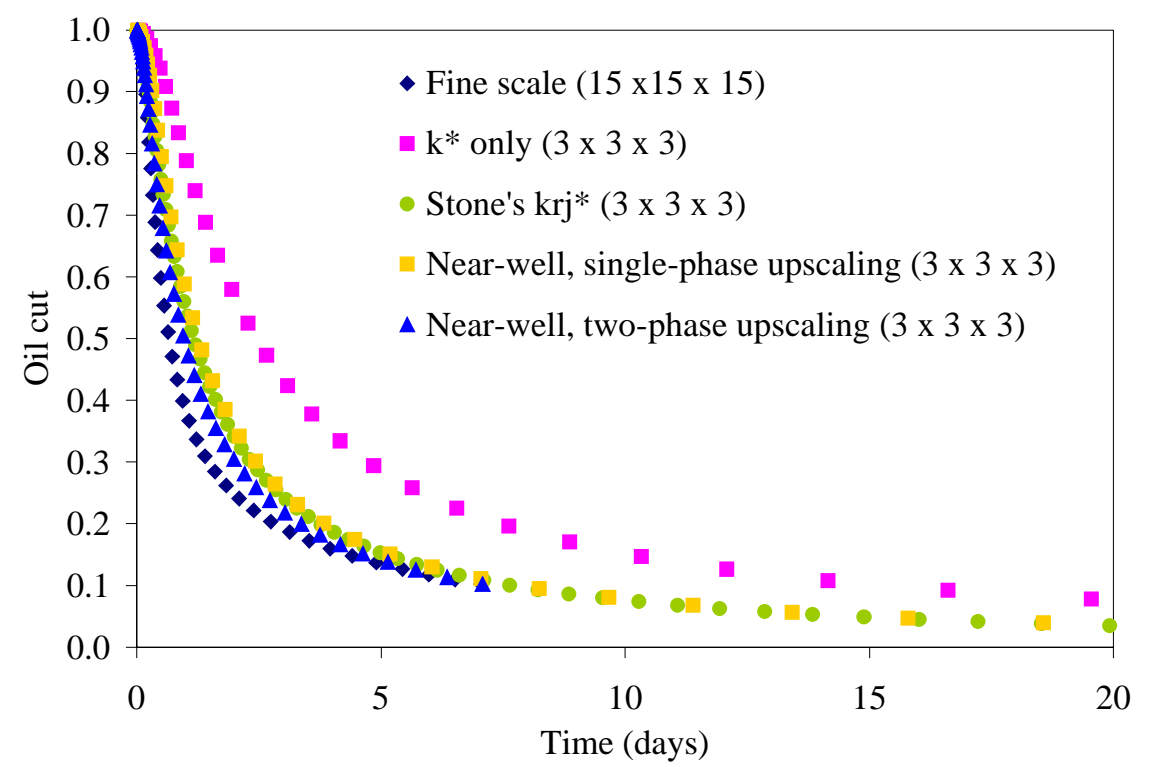

Figure 6-12: Local well model fractional flow curves for a 3D case, comparing the match to the fine scale solution given by different coarse models 


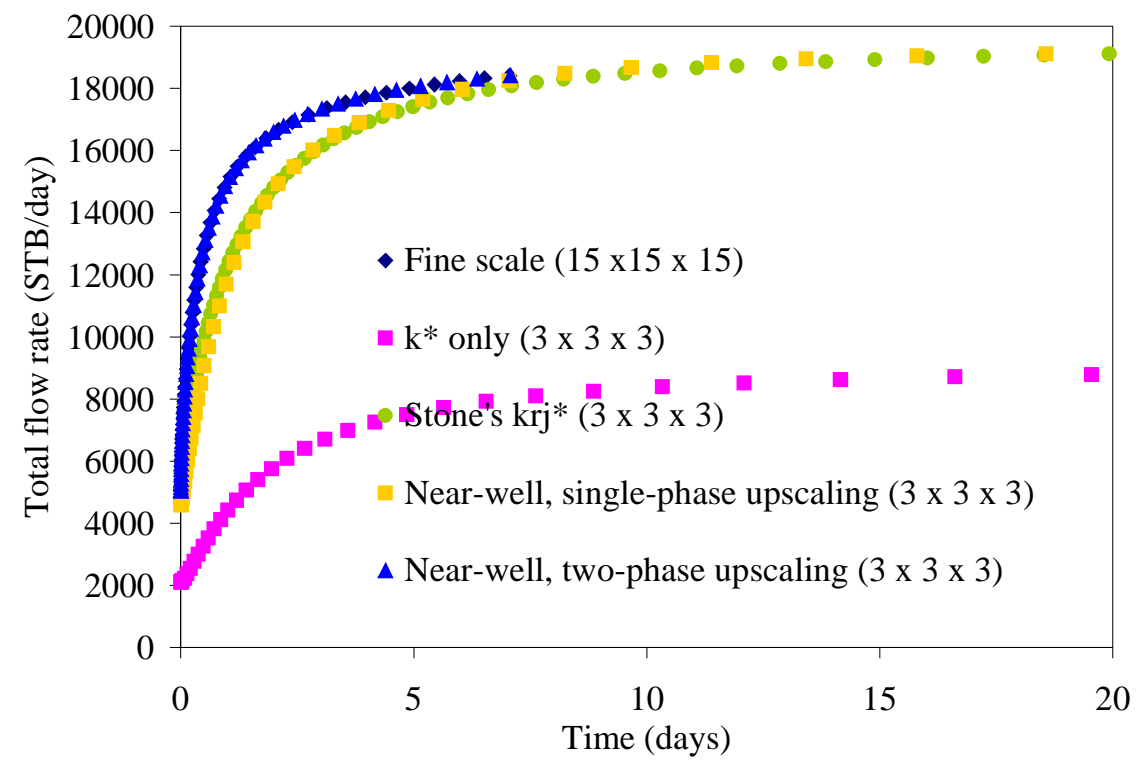

Figure 6-13: Local well model total flow rate curves for a 3D case, comparing the match to the fine scale solution given by different coarse models

\subsection{Upscaling to Radial Grids}

In previous work (Jenny et al., 2002), we applied multiblock grids (MBGs) to model nonconventional wells. Multiblock grids are well suited for nonconventional well modeling because they are globally unstructured and thus able to resolve relatively complex geometries. However, by maintaining local structure, these models can be handled more efficiently than fully unstructured models. In our approach, a MBG is conformed to a three dimensional "hole" through the grid of radius $r_{h}=a r_{w}$ (where $r_{w}$ is the wellbore radius and $a$ is a constant) that is centered around the well trajectory. By taking $a>>1$, the grid does not need to resolve down to the scale of the wellbore, resulting in computational savings. As an example, the MBG for a deviated well is illustrated in Fig. 6-14 (another example of a MBG was shown earlier in Fig. 4-10). The "hole well model" relates the pressure at the hole boundary $p_{h}$ (at radial location $r_{h}$ ) to the well pressure $p_{w}$ and to the cell centered pressure $p_{b}$ in the first ring of blocks (at radial location $r_{b}$ ):

$$
p_{h}=p_{w}+\left(p_{b}-p_{w}\right) \frac{\ln r_{h}-\ln r_{w}}{\ln r_{b}-\ln r_{w}}
$$

This model can be expressed in an equivalent form in terms of well transmissibilities $T^{w}$ : 


$$
q=T^{w}\left(p_{w}-p_{b}\right)=T_{h}^{w}\left(p_{w}-p_{h}\right)
$$

where:

$$
T^{w}=\frac{\theta k H}{\ln r_{b}-\ln r_{w}} \text { and } T_{h}^{w}=\frac{\theta k H}{\ln r_{h}-\ln r_{w}}
$$

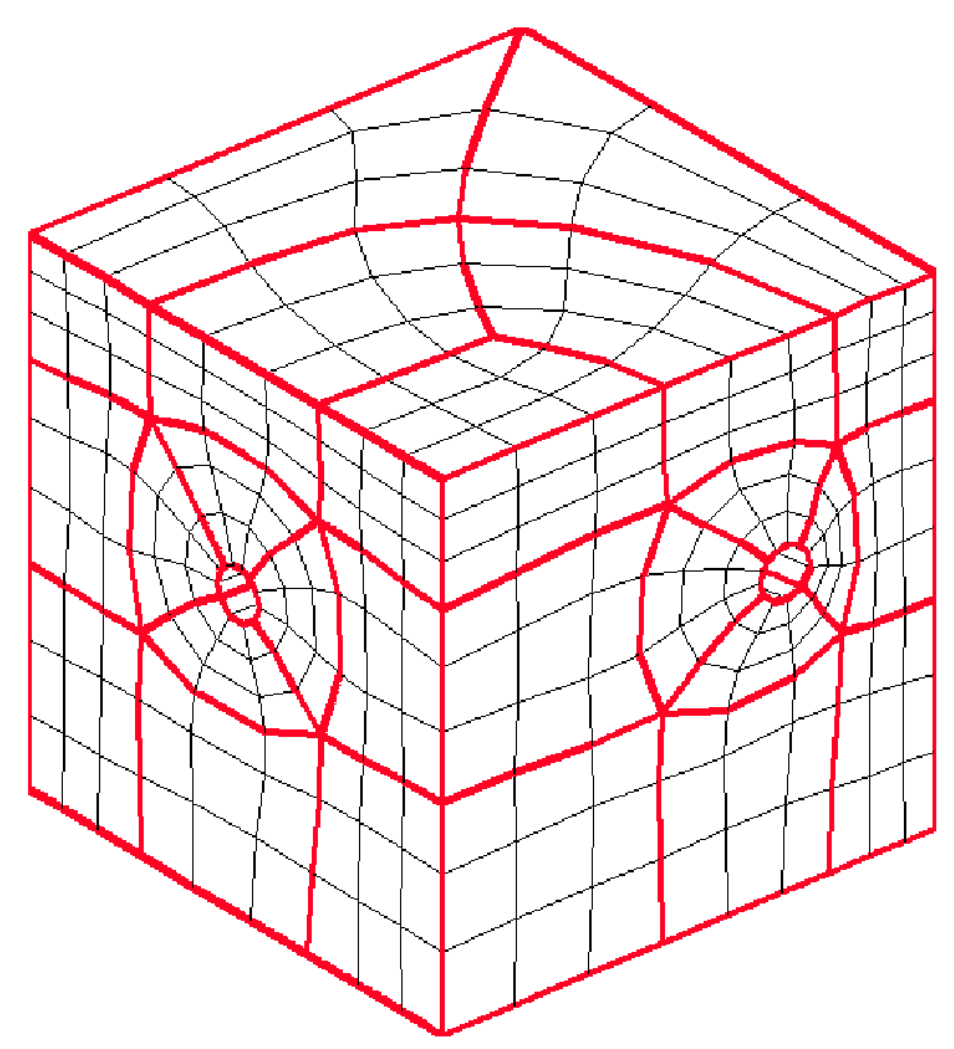

Figure 6-14: Multiblock grid for a deviated well

This model is valid for isotropic and relatively homogeneous systems. When the near-well permeability field displays significant heterogeneity or anisotropy, a model that accounts for these effects is required. Because we do not wish to grid down to the scale of the wellbore, these effects must be captured through an appropriate effectivization. This is accomplished using a near-well upscaling procedure, in which we solve a local problem in order to determine coarse scale parameters, as we now describe. 
The details of the near-well upscaling method are presented in Wolfsteiner and Durlofsky (2002). In contrast to the hole well model for homogeneous systems, where well transmissibilities were derived from the analytical single-phase pressure solution for radial flow, coarse scale well transmissibilities are now computed using an optimization procedure that forces the local coarse grid model to replicate the integrated response of the local fine scale solution. This is analogous to the approach applied above for Cartesian grids.

Fig. 6-15 shows the local coarse grid on the left (with the hole designated by the heavy dashed line) and the corresponding fine grid on the right, which is over the same local region but contains refinement down to the wellbore. Note that the coarse grid is constructed by removing coordinate lines from the fine grid. Properties on the coarse and fine grids are denoted by the superscripts $c$ and $f$ respectively.
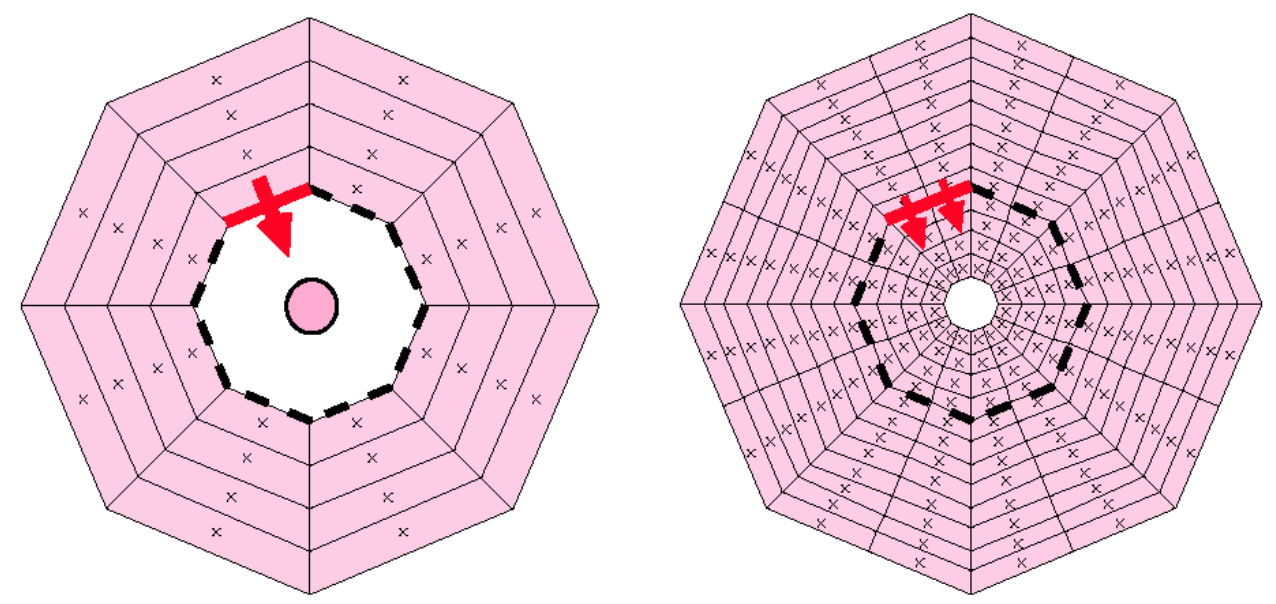

Figure 6-15: Schematic illustrating radial upscaling (hole and flux locations indicated): (a) $4 \times 8$ coarse grid with hole and (b) $12 \times 16$ fine grid resolved down to wellbore

For each coarse sector $j$, our intent is to compute the coarse scale well transmissibility $T_{h}^{w, c}$ such that flow from the well into the first ring of grid cells can be represented via:

$$
q^{c}=T_{h}^{w, c}\left(p_{w}-p_{h}^{c}\right)
$$

We compute the $T_{h}^{w, c}$ such that the flux into each of the coarse cells in the innermost ring is equal to the sum of corresponding fine fluxes computed from the detailed reference grid. Corresponding fluxes for one segment are indicated via arrows in Fig. 6-15. Since the $q^{c}$ for all $N_{j}$ sectors are 
coupled, we must minimize the objective function $E$, given by

$$
E=\sum_{j=1}^{N_{j}}\left|q^{c}-\sum q^{f}\right|_{j, r=r_{h}}
$$

to determine the $T_{h}^{w, c}$.

As demonstrated by Wolfsteiner and Durlofsky (2002), a successive substitution scheme can be used to determine the $T_{h}^{w, c}$. This usually requires relatively few iterations, with each iteration involving only coarse grid solutions. The method has so far been implemented for two dimensional systems, though we expect that the algorithm can be extended to the three dimensional case in a straightforward manner. As an alternative, using the existing implementation the three dimensional case can be treated as a series of two dimensional slices along the well trajectory.

\subsubsection{Multiblock Cross Section Example}

In order to assess the applicability of the near-well upscaling procedure to MBGs, we consider a MBG cross section extracted from Fig. 6-14. The coarse model in this case is two dimensional and has 12 blocks (Fig. 6-16a). The local fine model used to compute the coarse model parameters consists of the four blocks adjacent to the hole (each cell refined by $3 \times 3$ ) and four new blocks that are inserted to span the hole down to the well radius as shown in Fig. 6-16b.

For this example we perform simulations on four different grids, each of which is assigned permeability values from the same geostatistical fine grid. Following the procedure outlined above, we first simulate a single phase, steady-state problem on the fine near-well domain (Fig. 6-16b) to obtain the reference flux distribution at the (coarse) hole boundary. Several iterations on a coarse version of Fig. 6-16b (with the innermost four blocks removed) are then performed to obtain the upscaled near-well parameters.

The global simulation problem in this case is a two-phase flow with constant total mobility. The system is initially saturated with oil, but water flows in from the boundaries. The total drainage area is $328 \mathrm{ft} \times 328 \mathrm{ft}$. Detailed simulation properties are given in Lee et al. (2003). We simulate two coarse grid models - one with the standard hole well model and one with the upscaled well model. A final simulation run, which would be absent in an actual application but serves as a global reference for the upscaling procedure, is performed on a fully refined (fine 
grid) version of Fig. 6-16a (including additional hole blocks) of dimensions $16 \times(9 \times 9)$; i.e., 1296 cells in total.

In Fig. 6-17, the two coarse simulations and the global fine grid result are compared in terms of water cut in the production stream. Water encroaching from the right boundary breaks through after about 38 days for the fine grid result. The coarse simulation with a total of 108 cells using the standard well model exhibits a trend that is different from the fine grid. The coarse model with the upscaled near-well parameters reproduces the water cut behavior more accurately as compared to the standard coarse model. The water cuts after 100 days are $0.41,0.25$ and 0.38 for the fine reference, coarse standard and coarse upscaled models respectively. In terms of total liquid production (Fig. 6-18), both coarse grid profiles exhibit a deviation from the fine grid solution at very early time. However, the coarse grid model with upscaled near-well parameters is in much closer agreement with the fine solution after the transient period.

This example demonstrates the applicability of the near-well upscaling to the MBG context. Using this approach, we are able to include effects down to the scale of the wellbore in large scale flow simulations via appropriately computed coarse scale parameters. Due to the flexibility of the multiblock approach, the model can retain a near radial grid structure along the nonconventional well trajectory.
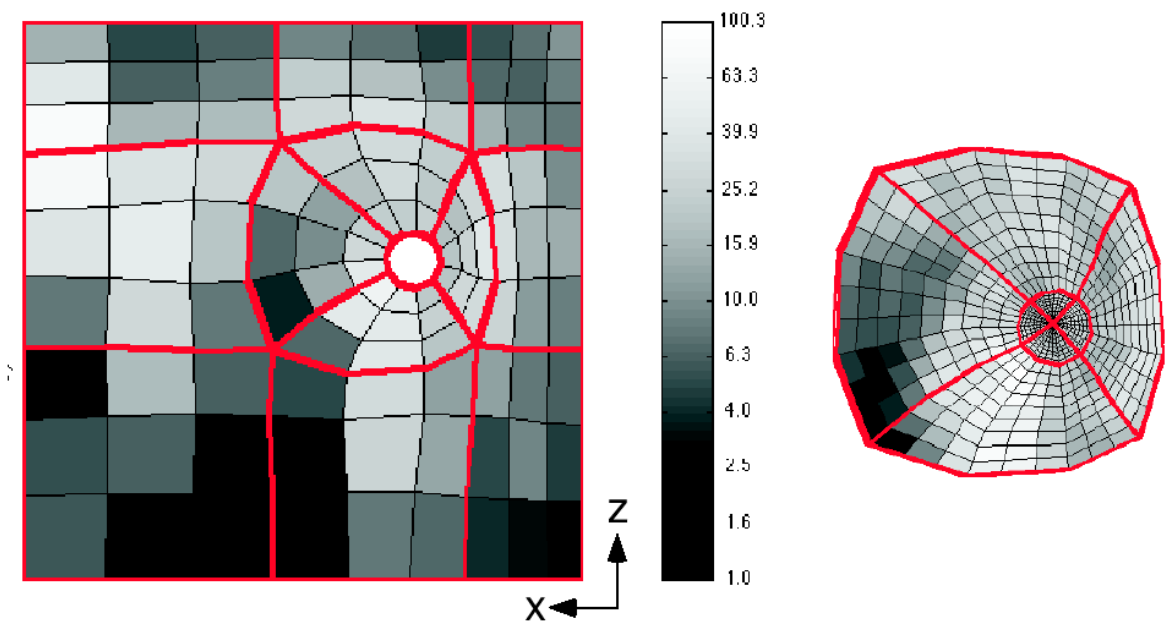

Figure 6-16: Multiblock grid and permeability field: (a) coarse grid with 12 blocks (each $3 \times$ $3)$ and (b) near-well fine grid with 8 blocks $($ each $9 \times 9)$ resolved down to the wellbore 


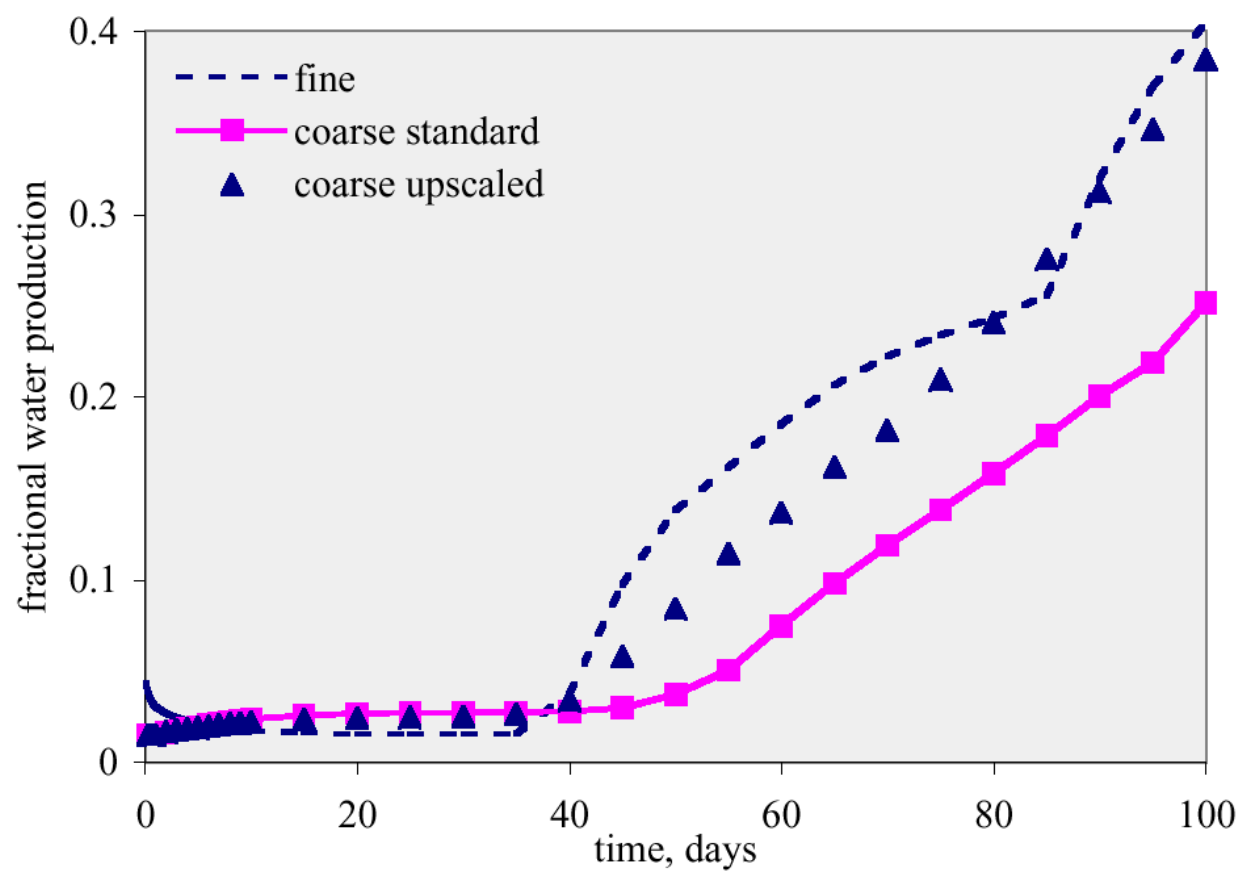

Figure 6-17: Comparison of producing water cut

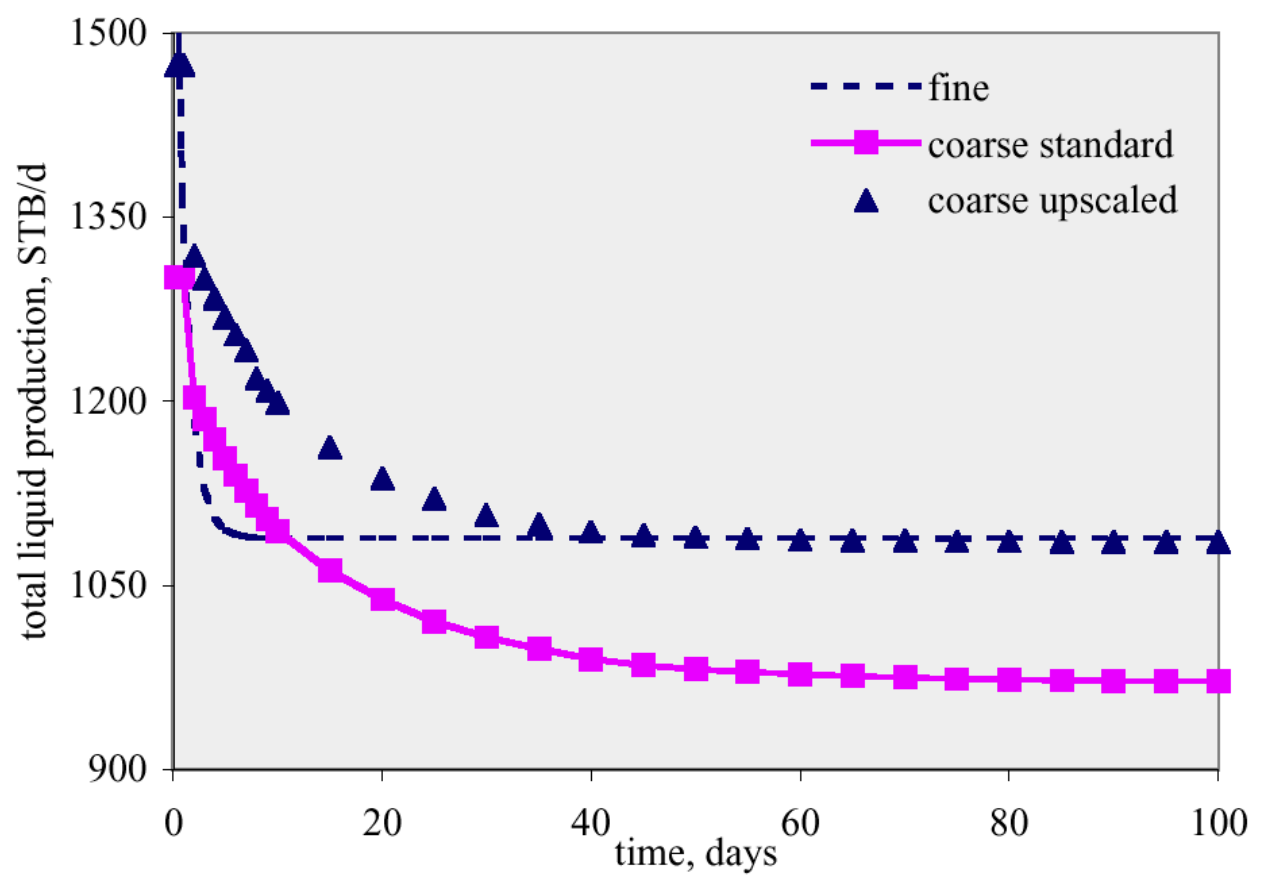

Figure 6-18: Comparison of total liquid production 


\subsection{Summary}

In this chapter we presented single-phase and two-phase near-well upscaling procedures for structured simulation models and a single-phase upscaling technique applicable for the near-well region within a multiblock (globally unstructured) simulation context. This work can be summarized as follows:

- Standard upscaling procedures (based on the calculation of $\mathbf{k}^{*}$ from a linear flow problem) may not be appropriate for use in the near-well region. An upscaling procedure that is based on a radial flow problem can provide better accuracy in coarse scale models.

- The near-well upscaling procedures use a local well model extracted from the global simulation model. For single-phase parameter upscaling, an effective well index and well block transmissibilities are computed. For two-phase parameter upscaling, which is required when near-well multiphase effects are important, upscaled well block (pseudo) relative permeabilities are additionally determined.

- Both the single-phase and two-phase parameters are most accurately computed by optimizing over the coarse local well model such that the coarse model response matches the averaged response of the fine model.

- A single-phase parameter upscaling procedure was defined for nearly radial grids, as might be used within a multiblock simulation context. Near-well upscaling in this case enables the use of grids that do not need to resolve down to the scale of the wellbore.

- All of the methods described here were applied to example cases. Improvement over standard procedures was demonstrated in essentially all cases. 


\subsection{References}

Aziz, K., Arbabi, S. and Deutsch, C.V.: "Why is it so Difficult to Predict the Performance of Horizontal Wells?” JCPT, 37-45, Oct. 1999.

Barker, J.W. and Dupouy, P.: "An Analysis of Dynamic Pseudo-Relative Permeability Methods for Oil-Water Flows,” Petroleum Geoscience, 5, 385-394, 1999.

Bishop, C.M.: Neural Networks for Pattern Recognition, Oxford University Press, New York, 1995.

Chen, Y., Durlofsky, L.J., Gerritsen, M. and Wen, X.H.: "A Coupled Local-Global Upscaling Approach for Simulating Flow in Highly Heterogeneous Formations," Adv. Water Resour., 26, 1041-1060, 2003.

Darman, N.H., Pickup, G.E. and Sorbie K.S.: “A Comparison of Two-Phase Dynamic Upscaling Methods Based on Fluid Potentials," Comput. Geosciences, 6, 5-27, 2002.

Deutsch, C.V. and Journel, A.G.: GSLIB: Geostatistical Software Library and User's Guide, 2nd edition, Oxford University Press, 368 p, 1998.

Ding, Y.: "Scaling-up in the Vicinity of Wells in Heterogeneous Field," paper SPE 29137 presented at the SPE Symposium on Reservoir Simulation, San Antonio, Feb. 12-15, 1995.

Ding, Y. and Urgelli, D.: "Upscaling of Transmissibility for Field Scale Flow Simulation in Heterogeneous Media," paper SPE 38016 presented at the SPE Symposium on Reservoir Simulation, Dallas, June 8-11, 1997.

Durlofsky, L.J.: "Numerical Calculation of Equivalent Grid Block Permeability Tensors for Heterogeneous Porous Media," Water Resour. Res., 27, 699-708, 1991.

Durlofsky, L.J.: "Upscaling of Geocellular Models for Reservoir Flow Simulation: A Review of Recent Progress," Proceedings of the $7^{\text {th }}$ International Forum on Reservoir Simulation, Bühl/Baden-Baden, Germany, June 23-27, 2003.

Durlofsky, L.J., Jones, R.C. and Milliken, W.J.: "A Nonuniform Coarsening Approach for the Scale Up of Displacement Processes in Heterogeneous Porous Media," Adv. Water Resour., 20, 335-347, 1997.

Durlofsky, L.J., Milliken, W.J. and Bernath, A.: "Scaleup in the Near-Well Region,” SPEJ, 110117, March 2000.

Emanuel, A.S. and Cook, G.W.: "Pseudo Relative Permeability for Well Modeling," SPEJ, 14, 7-9, 1974.

Jenny, P., Wolfsteiner, C., Lee, S.H. and Durlofsky, L.J.: "Modeling Flow in Geometrically Complex Reservoirs Using Hexahedral Multiblock Grids,” SPEJ, 149-157, June 2002.

Lee, S.H., Wolfsteiner, C., Durlofsky, L.J., Jenny, P. and Tchelepi, H.A.: "New Developments in Multiblock Reservoir Simulation: Black Oil Modeling, Nonmatching Subdomains and Near- 
Well Upscaling," paper SPE 79682 presented at the SPE Reservoir Simulation Symposium, Houston, Feb. 3-5, 2003.

Lemouzy, P.: "Quick Evaluation of Multiple Geostatistical Models Using Upscaling with Coarse Grids: A Practical Study," presented at the Fourth International Reservoir Characterization Technical Conference, Houston, March 2-4, 1997.

Mascarenhas, O., 1999. Accurate Coarse Scale Simulation of Horizontal Wells. Master's report, Stanford University.

Mascarenhas, O. and Durlofsky, L.J.: "Coarse Scale Simulation of Horizontal Wells in Heterogeneous Reservoirs,” J. Pet. Sci. and Eng., 25, 135-147, 2000.

Muggeridge, A.H., Cuypers, M., Bacquet, C. and Barker, J.W.: "Scale-up of Well Performance for Reservoir Flow Simulation,” Petroleum Geoscience, 8, 133-139, 2002.

Peaceman, D.W.: "Interpretation of Well-Block Pressures in Numerical Reservoir Simulation with Nonsquare Grid Blocks and Anisotropic Permeability," SPEJ, 531-543, June 1983.

Pickup, G.E., Ringrose, P.S., Jensen, J.L. and Sorbie, K.S.: "Permeability Tensors for Sedimentary Structures," Math. Geol., 26, 227-250, 1994.

Renard, Ph. and de Marsily, G.: "Calculating Equivalent Permeability: A Review," Adv. Water Resour., 20, 253-278, 1997.

Stone, H.L.: "Rigorous Black Oil Pseudo Functions," paper SPE 21207 presented at the SPE Symposium on Reservoir Simulation, Anaheim, Feb. 17 - 20, 1991.

Wen, X.-H. and Gomez-Hernandez, J.J.: "Upscaling Hydraulic Conductivities in Heterogeneous Media: An Overview," J. Hydrol., 183, ix-xxxii, 1996.

Wolfsteiner, C. and Durlofsky, L.J.: "Near-Well Radial Upscaling for the Accurate Modeling of Nonconventional Wells," paper SPE 76779 presented at the SPE Western Regional Meeting, Anchorage, May 20-22, 2002. 


\section{Optimization of Nonconventional Well Type and Operation}

Advanced or nonconventional wells include multilateral and "smart" wells; i.e., wells equipped with downhole sensors and valves. Advanced wells are used in a variety of field settings, though the selection of the optimum type of well and the determination of the optimal placement and operation of these wells remain significant challenges. In this chapter, we first discuss and illustrate our recent work on the optimization of nonconventional well type and placement. We then describe procedures for the optimization of smart well operation, particularly the linkage of the optimization with history matching.

The optimization of nonconventional well type and placement is very complicated due to the vast number of possible well configurations (i.e., many possible values for the location, orientation and length of the mainbore as well as the number, location, length and orientation of laterals). The problem is further complicated because of uncertainty in the reservoir geology. We address this problem using a genetic algorithm, which is a stochastic optimization procedure that qualitatively mimics Darwinian natural selection. Our implementation, described in detail by Yeten (2003) and Yeten et al. (2003), entails a particular coding of the unknowns on the "chromosome" that allows the type of well (e.g., monobore, bilateral, trilateral) to evolve during the course of the simulation.

Optimization of well placement has been studied previously by a number of researchers. Seifert et al. (1996) developed a methodology for locating horizontal and highly deviated wells. Their approach attempts to find the well trajectory that penetrates the most productive geological units. Bittencourt and Horne (1997) optimized the placement of multiple vertical and horizontal wells using a hybrid optimization algorithm that consisted of GA, polytope, and Tabu search methods in conjunction with a numerical simulator. Santellani et al. (1998) presented an automatic well location estimation algorithm using GAs. Centilmen et al. (1999) developed a neuro-simulation technique that forms a bridge between a reservoir simulator and a predictive artificial neural network. They selected several key well scenarios, either randomly or by intuition, for network training. Following the training step, numerous well scenarios could be evaluated efficiently. Güyagüler et al. (2002) applied a hybrid optimization algorithm that utilized a GA, a polytope method, kriging and an artificial neural network (used as proxies for 
the function evaluations), along with a reservoir simulator. Güyagüler et al. optimized up to four vertical water injection well locations for a real field waterflood project. In related work, Güyagüler and Horne (2001) introduced a utility function approach to account for the reservoir uncertainty. Montes et al. (2001) also used a GA to optimize the locations of vertical production and injection wells.

Our work differs from previous developments in that we implement a methodology to optimize the type (e.g., number of laterals), location, and trajectory of general nonconventional (rather than monobore) wells. Our approach can also account for uncertainty in the reservoir description, which was not considered in most previous studies (Güyagüler and Horne, 2001, is an exception). We additionally introduce several specific helper algorithms to accelerate the search.

We next consider the optimization of smart well control. Our basic optimization procedure, described in detail in Yeten et al. (2004), entails the application of a conjugate gradient algorithm that uses a reservoir simulator as a function evaluator. The method provides valve settings that optimize the specified objective function (typically cumulative oil) for a particular reservoir geology. The approach in Yeten et al. (2004) is more appropriate for use in screening (i.e., for decisions regarding smart well deployment) rather than as a reservoir management tool as it does not utilize the real-time data supplied by the downhole sensors. In an actual application, this sensor data can be employed to continuously update the geological model and this updated model can then be used for the valve optimization.

We have also applied decision analysis techniques to decide whether or not to deploy a smart well (Yeten et al., 2004). This approach accounted for geological uncertainty as well as hardware uncertainty; i.e., the possibility of valve failure at different times in the life of the project. Hardware uncertainty was modeled using a Weibull failure model, which allows the failure likelihood to vary in time. Various valve failure modes were also considered. This study demonstrated that downhole control can compensate to some extent for geological uncertainty, even when the possibility of equipment failure is included. This demonstrates the insurance value of smart completions.

In the work presented here, we integrate valve optimization and history matching algorithms to accomplish a prototype real-time strategy. In our example cases, downhole sensor data are 
assumed to provide inflow rates for each fluid phase in each instrumented branch of a multilateral well. These data are generated by simulating production from one particular geostatistical realization. We apply the overall history matching - well control optimization algorithm to cases involving a quadlateral well producing in a channelized reservoir with a gas cap and aquifer. It is shown that the combined procedure is capable of increasing cumulative production significantly, to levels very near those obtained when the geology is assumed to be known. Because history-matched models are nonunique, there is potential benefit in optimizing valve settings over multiple history-matched models. We investigate this issue and demonstrate that the use of multiple history-matched models does provide significant improvement over optimization using just a single history-matched model in some cases.

There are a number of other research groups addressing the optimization of smart well performance. Yeten and Jalali (2001) and Sinha et al. (2001), for example, demonstrated the applicability of smart wells in practical settings. Existing smart well control procedures can be classified as "reactive" control (in which valves are used to react to problems such as early water breakthrough after they occur) and "defensive" or "proactive" control (in which optimization procedures are used to anticipate and thereby mitigate problems). This latter type of control is preferable, as it is forward looking. Several defensive control procedures based on optimal control theory have been presented (e.g., Sudaryanto and Yortsos, 2000; Brouwer and Jansen, 2002). Our approach can be classified as a defensive control procedure, but it differs from these other techniques as we use numerical gradients (rather than those computed from an adjoint procedure) in our optimization. This is less efficient computationally but allows us to use an existing simulator with a sophisticated multisegment well model (Holmes et al., 1998). The linkage of history matching and smart well optimization procedures for realistic (channelized) reservoirs does not appear to have been accomplished previously.

In this chapter, we first describe and apply genetic algorithms for the optimization of nonconventional well type, location and trajectory. Geological uncertainty is included in one of the example cases. We then present our combined smart well optimization and history matching procedure. Results are presented for two different channelized reservoirs. 


\subsection{Genetic Algorithm for Optimization of Well Type and Trajectory}

The genetic algorithm (GA) applied for the optimization of well type and trajectory was described previously by Yeten et al. (2003). The basic approach entails the encoding of well type and trajectory information on binary strings or "chromosomes." A "population" of "individuals" (each individual is encoded on a chromosome and represents a particular well or well configuration) evolves from one "generation" (iteration) to the next. The "fitness" of each individual $i$ is simply the value of the objective function $f_{i}$ (e.g., net present value or cumulative oil) that it provides. Selection and crossover operations enable the best individuals (up to that point) to be combined (with some degree of randomness), the idea being that improved solutions have some probability of appearing. Mutation operations are also applied; these introduce a further component of randomness into the procedure. The encoding of information on the chromosome is done in a way that allows the type of well (e.g., monobore, multilateral) to evolve over the course of the GA optimization.

The GA optimization is very intensive computationally, so a number of procedures to accelerate the calculations are introduced. These include the use of a near-well upscaling procedure in which an effective skin accounting for subgrid heterogeneity is computed (along the lines described in Chapter 4) for use with an upscaled simulation model. Proxies such as artificial neural-networks are also introduced to reduce the amount of computation required. Another of the helper tools applied here is an evaluation-only search method, referred to as a hill climber, which is a heuristic adaptation of the Hooke-Jeeves pattern search algorithm (Reed and Marks, 1999). This procedure is applied only in the immediate vicinity of the solution.

The number of parameters to be optimized increases as the complexity of the well (i.e., number of junctions and laterals) increases. In our procedure, the main bore is not considered to be perforated if one or more laterals emanate from it. If there are no laterals, the main bore is taken to be fully perforated. The main bore diameter, designated $d_{\text {well }}$, and the target production rate or target bottomhole pressure, designated $q$, can also be optimized along with the type, location, and trajectory of the well. If the diameter of the main bore is to be optimized, then the lateral diameter is selected based on the main bore diameter.

The vector of parameters to be optimized, designated $\mathbf{p}$, is given by: 


$$
\mathbf{p}=\left(\left[\begin{array}{c}
h_{x} \\
h_{y} \\
h_{z} \\
l_{x y} \\
\theta \\
t_{z}
\end{array}\right]\left[\begin{array}{c}
J^{1} \\
l_{x y}^{1} \\
\theta^{1} \\
t_{z}^{1}
\end{array}\right] \ldots\left[\begin{array}{c}
J^{k} \\
l_{x y}^{k} \\
\theta^{k} \\
t_{z}^{k}
\end{array}\right] \quad q \quad d_{\text {well }}\right)^{T}
$$

The first column of $\mathbf{p}$ represents the main bore; subsequent columns correspond to the $k$ laterals, well settings, and hole diameter. Here $h_{x}, h_{y}$ and $h_{z}$ designate the location of the heel of the main bore, $l_{x y}$ is the length of trajectory projected onto the $x-y$ plane, $\theta$ is the orientation of the well in the $x-y$ plane and $t_{z}$ is the depth to the trajectory endpoint $\left(l_{x y}, \theta\right.$ and $t_{z}$ together define the toe of the main bore). Laterals are represented in terms of their junction point $(J)$ on the main bore, quantified via the fractional length along the mainbore from where the lateral emanates, and $l_{x y}$, $\theta$, and $t_{z}$ for the lateral. When a lateral shares a junction with another lateral, $J$ for the subsequent lateral(s) is dropped from $\mathbf{p}$. See Yeten et al. (2003) for further details.

The optimization problem can now be represented by

$$
\operatorname{maximize} \underset{\text { constraints }}{\{f(\mathbf{p})\}}
$$

where $f$ is the objective function. Although our approach allows for both the minimization and maximization of $f$, we will concentrate on maximization problems. The objective function is either the cumulative oil production of the field or the net present value (NPV) of the project. In the latter case, $f$ is defined as follows:

$$
f=\sum_{n=1}^{Y}\left(\frac{1}{(1+i)^{n}}\left[\begin{array}{c}
Q_{o} \\
Q_{g} \\
Q_{w}
\end{array}\right]_{n}^{T} \cdot\left[\begin{array}{c}
C_{o} \\
C_{g} \\
C_{w}
\end{array}\right]_{n}\right)-C_{\text {well }}
$$

where $Q_{p}$ is the production of phase $p$ during year $n, C_{p}$ is the profit or loss associated with this production, and subscripts $o, g$, and $w$ designate oil, gas, and water. The production is obtained from the reservoir simulator. The quantity $i$ represents the annual interest rate (APR), $Y$ is the total number of years and $C_{\text {well }}$ is the cost of drilling and completing the well. This cost can vary significantly depending on the field location and conditions. 
For purposes of this study, we represent $C_{\text {well }}$ as follows:

$$
C_{\text {well }}=\sum_{k=0}^{N_{\text {lat }}}\left[A \cdot d_{\text {well }} \cdot \ln \left(l_{\text {well }}\right) \cdot l_{\text {well }} \cdot(2-\alpha)\right]_{k}+\sum_{k=1}^{N_{\text {jum }}} C_{\text {jun }}
$$

This is an approximate formula, though it is based on cost figures obtained for a real onshore field. In Eq. 7-4, $k=0$ represents the main bore, $k>0$ represents the laterals, $d_{\text {well }}$ is the diameter of the main bore (in $\mathrm{ft}$ ), and $C_{\mathrm{jun}}$ is the cost of milling the junction. The parameter $A$ is a constant that contains conversion factors and represents the specific costs related to the field location and conditions. In our examples we set $A=12$. The parameter $\alpha$ accounts for the inclination of the well and is given by $\alpha=\left(h_{z}-t_{z}\right) / l_{\text {well }}$. The contrast between the cost of drilling a vertical well and a horizontal well is captured by the term $(2-\alpha)$ in Eq. 7-4. For vertical wells, $\alpha=1$, while for horizontal wells $\alpha=0$, which means that a horizontal well will be twice as expensive as a vertical well on a per length basis.

A schematic of the overall optimization procedure is shown in Fig. 7-1. The relationship between the GA and the helper algorithms, and the basic way in which the optimization proceeds, is depicted in this figure. The fitness of a particular well is simply the value of the resulting objective function determined from Eqs. 7-3 and 7-4.

In practice, the reservoir geology is known in only a probabilistic sense, so the optimization should account for this uncertainty. This can be accomplished within the GA framework by optimizing over multiple realizations using a prescribed risk attitude (Güyagüler and Horne, 2001; Yeten et al., 2003). For example, for an ensemble of $M$ realizations, we can define an objective function $F_{i}$ for an individual (well) $i$ over the ensemble as follows:

$$
F_{i}=\langle f\rangle_{i}+r \sigma_{i}
$$

where $r$ is the risk attitude and $\sigma_{i}$ is the standard deviation. Here $\sigma_{i}$ is computed as:

$$
\sigma_{i}=\left(\frac{1}{M} \sum_{j=1}^{M}\left[f_{i j}-\langle f\rangle_{i}\right]^{2}\right)^{1 / 2}
$$

where $j$ designates a particular realization and $f_{i j}$ is the value of the objective function for well $i$ in realization $j$. A value of $r=0$ indicates a risk neutral attitude, while $r<0$ indicates a risk 
averse attitude and $r>0$ a risk seeking attitude. Using this formulation we can find the well that is optimal (in the sense of Eq. 7-5) for an ensemble of geological realizations.

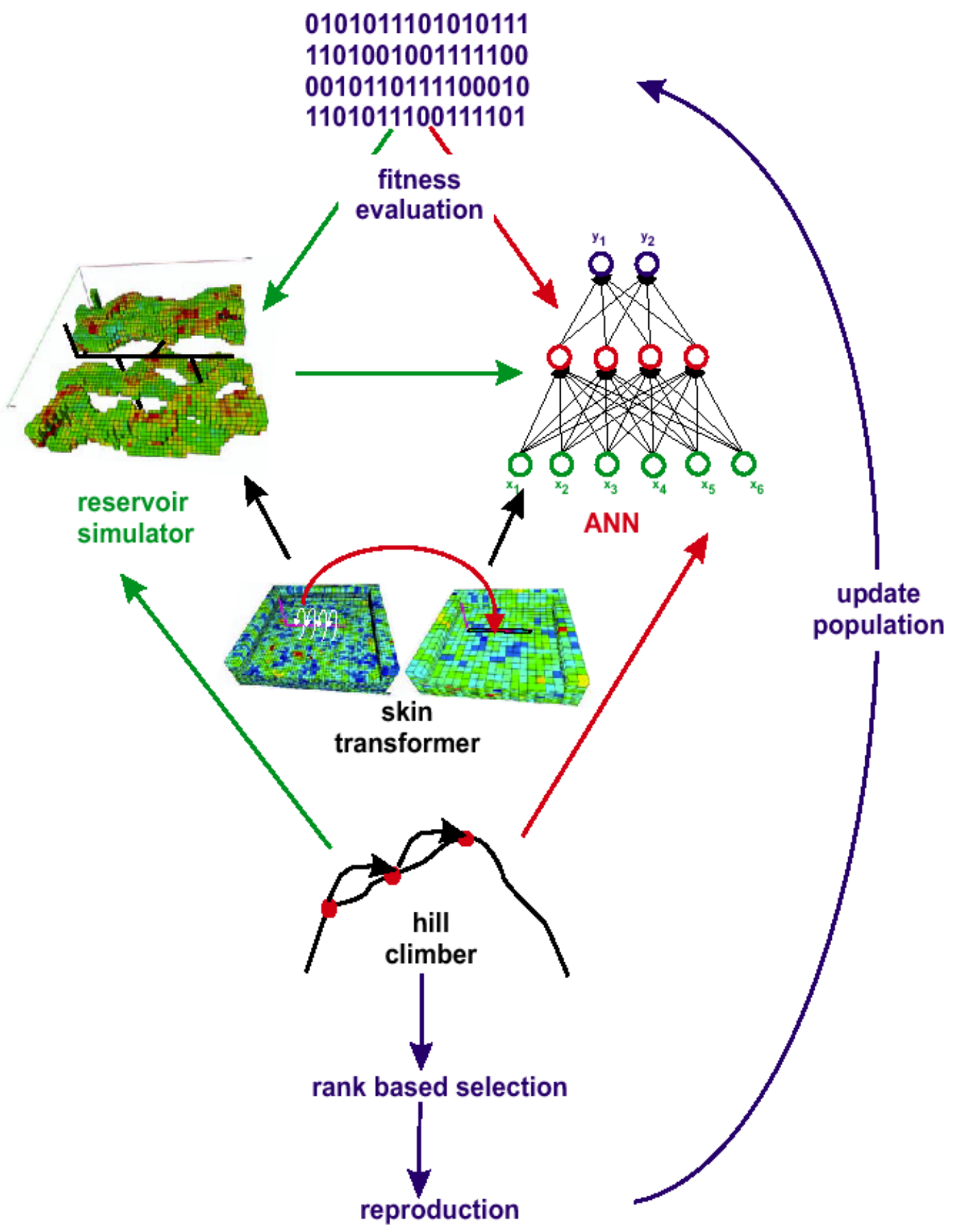

Figure 7-1: Schematic of overall optimization procedure 


\subsection{Optimum Well Determination - Examples}

\subsubsection{Deterministic Geological Model}

We first demonstrate the performance of the GA algorithm for a particular (deterministic) geological model. This case involves a dual-drive reservoir. We introduce a gas cap of large pore volume at the top of the reservoir and an aquifer at the bottom. The bubblepoint pressure of the system, which corresponds to the pressure at the bottom of the gas cap $(5,000 \mathrm{ft})$, is 4,000 psi. The permeability field was generated using an unconditioned sequential Gaussian simulation (Deutsch and Journel, 1998) on a $50 \times 50 \times 21$ simulation grid. Dimensionless correlation lengths of $0.5,0.5$, and 0.05 were used in the $x, y$, and $z$ directions, respectively (correlation length was nondimensionalized by the system length in the corresponding direction). The ratio of vertical to horizontal permeability for each gridblock was set to 0.1. More details on the reservoir and fluid properties, as well as economic parameters, can be found in Yeten et al. (2003).

We upscaled this reservoir model to a $20 \times 20 \times 11$ grid using the near-well (effective skin) upscaling procedure described above. Although this upscaling ratio is relatively small, the coarsened model ran almost 100 times faster than the fine model, because of some convergence problems encountered in simulations of the fine model. Two main economic constraints were used for this optimization. Specifically, the well was shut in if the water cut exceeded $95 \%$ and the production rate of the well was cut back by $10 \%$ whenever the production gas/oil ratio (GOR) exceeded $10 \mathrm{MSCF} / \mathrm{STB}$.

We allow the optimization procedure to consider multilateral wells with up to four laterals, as well as monobore wells, within the same population. The objective in this case is to maximize NPV. The progress of the optimization is shown in Fig. 7-2. A target liquid rate of 10 MSTB/d was found to be the optimum. The NPV of the best well improves by about $34 \%$ from the first to the last generation, representing an increase of about $\$ 48$ million. The optimum well in this case is a quadlateral, as shown in Fig. 7-3. The well contacts a large reservoir area while avoiding proximity to the gas cap and aquifer. The evolution of the well types is presented in Fig. 7-4, where we show the number of each type of well (i.e., monobore, monolateral, bilateral, trilateral and quadlateral) in each generation. We start with equal numbers of each well type. Toward the end of the optimization, the quadlateral wells, which are optimal for this case, dominate the population. 
The effect of rejuvenation at every tenth generation is evident in Fig. 7-4. For example, at the tenth generation, rejuvenated wells are mostly trilaterals, because this is the optimal well type at this stage of the optimization. By the twentieth generation, however, the quadlaterals are predominant, because they performed the best between the tenth and twentieth generations. Because of our specialized representation of the different well types on the chromosomes, trilaterals can evolve into quadlaterals during the reproduction operations.

The "invalid" well type shown in the figure indicates wells that did not honor the constraints. In later generations, the number of invalid wells is quite high (almost half of the population) because of the fact that complex well trajectories (trilaterals and quadlaterals) are more likely to violate the constraints. This is largely because it is more difficult to fit these complex wells into the simulation grid. In addition, the probability of laterals intersecting each other increases with the number of laterals. Invalid wells are identified efficiently by the algorithm and do not cause a degradation in the performance of the optimization.

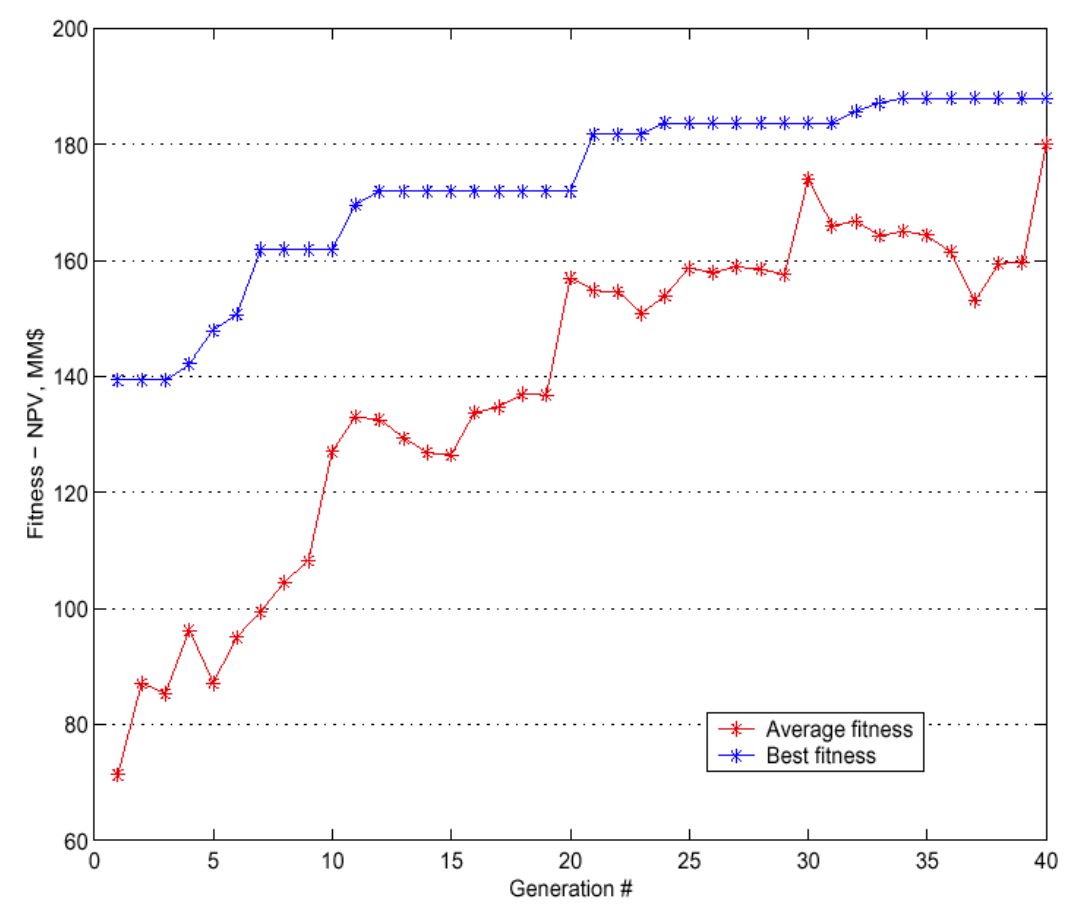

Figure 7-2: Progress of the optimization procedure 


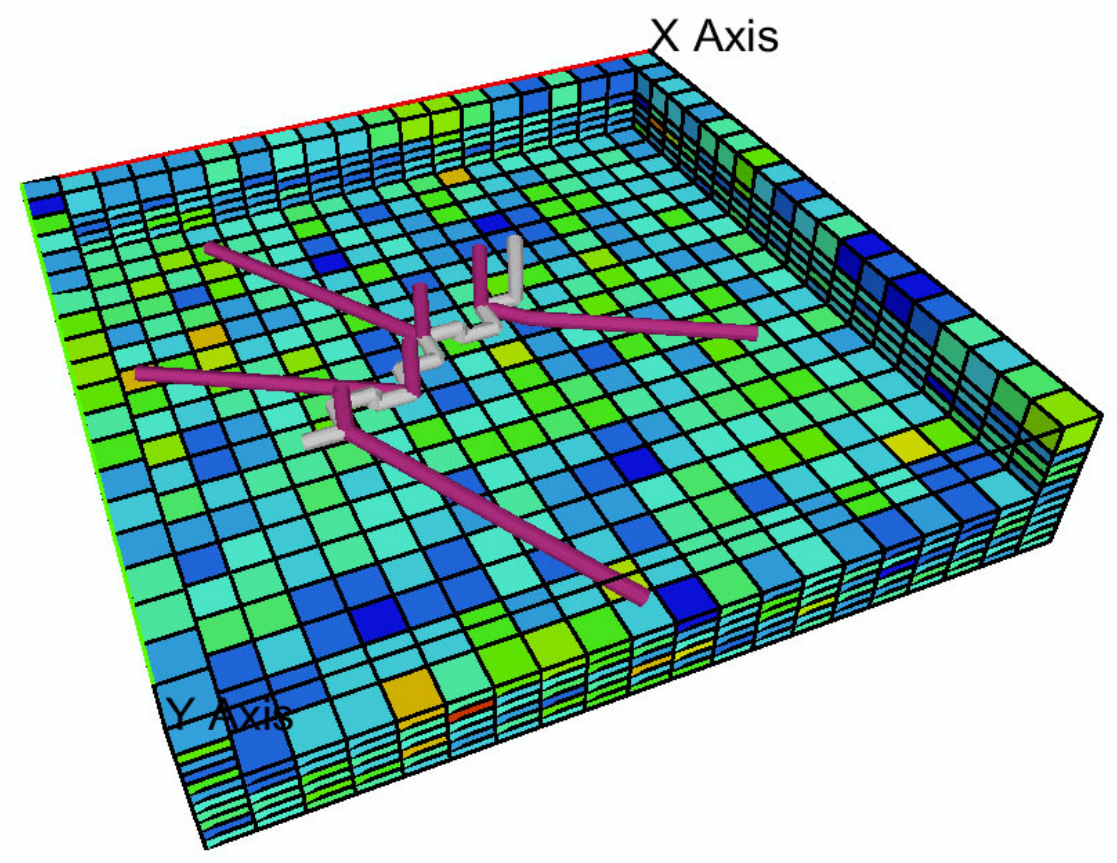

Figure 7-3: Optimum well (quadlateral) after 40 generations

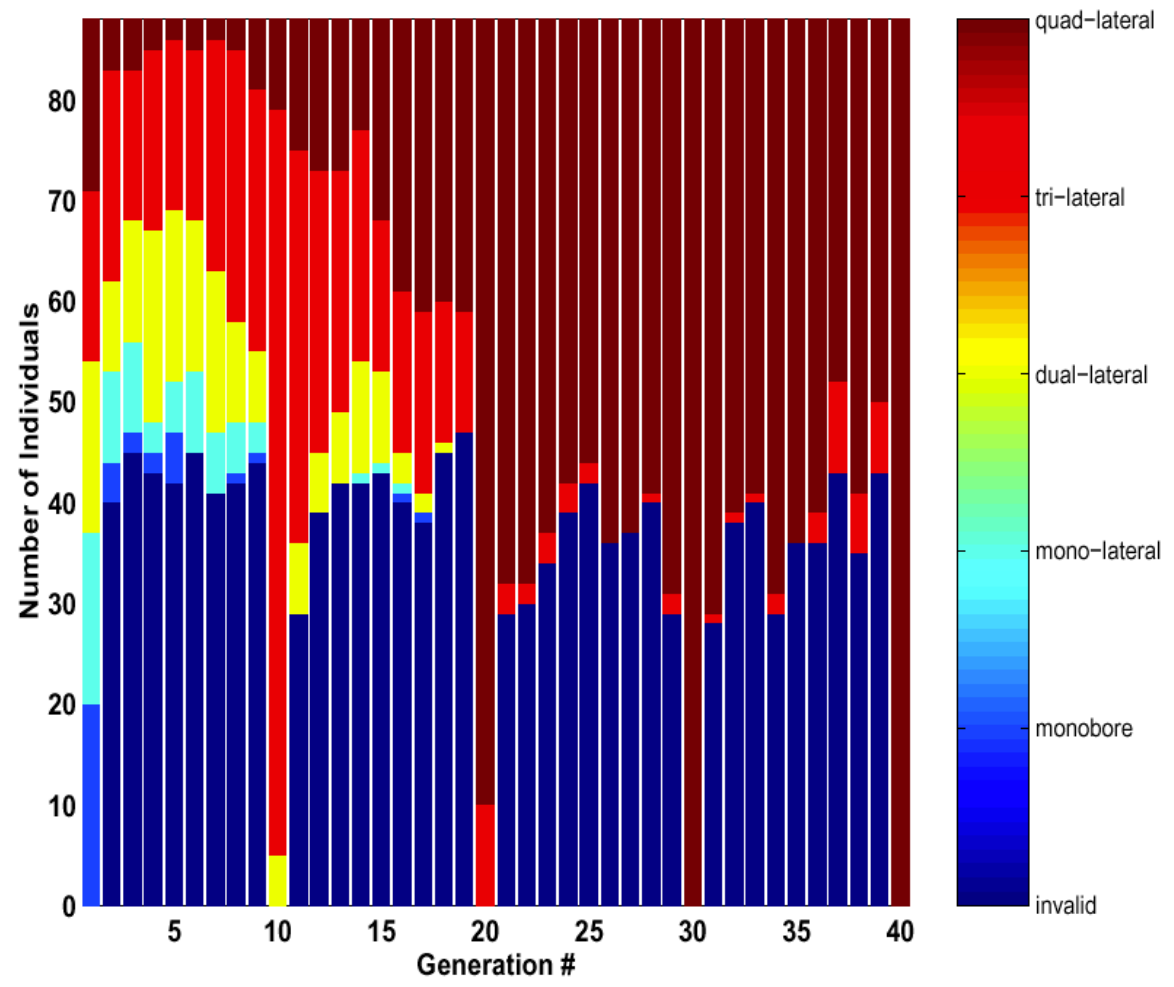

Figure 7-4: Variation of well types with generation (rejuvenation every ten generations) 


\subsubsection{Uncertain Geological Model}

We now illustrate the performance of the GA procedure with geological uncertainty. We consider five realizations of a channelized reservoir. Each realization was conditioned to data from three vertical observation wells. The channel sand was of average horizontal permeability 2900 md while the mudstone was of average horizontal permeability 1.2 md (vertical permeabilities were a factor of 10 less for both facies). Porosity was constant and equal to 0.2. Oil compressibility was set to $3 \times 10^{-5} \mathrm{psi}^{-1}$ and the formation volume factor was 1.3 . The physical size of the system was $4500 \mathrm{ft} \times 4500 \mathrm{ft} \times 100 \mathrm{ft}$ and the simulation models were of dimension $30 \times 30 \times 5$. Only primary production was considered and the time frame for the simulation was limited to one year. Risk attitudes of both $r=0$ and $r=-0.5$ were used.

The GA simulations included 60 individuals and converged after 32 generations $(r=0)$ and 20 generations $(r=-0.5)$. Monobore wells and multilaterals with up to four branches were considered. No proxies were applied. We used net present value (NPV) as the objective function, with reasonable costs assigned for the main bore, junctions and laterals (see Yeten et al., 2003). Fig. 7-5 shows the permeability field for one of the realizations (realization \#5) and the optimal well for (a) $r=0$ and (b) $r=-0.5$. In both cases the optimal well is a bilateral, though it is evident from the figure that the optimal well differs for the two cases. The well location in Fig. 7-5a does not appear to be ideal for this realization, though inspection of the other realizations indicates that the well intersects more channels in those systems.

Results for net present value for each realization are shown in Fig. 7-6. For $r=0$, the expected NPV is $\$ 3,510,000$ and the standard deviation is $\$ 936,000$, while for $r=-0.5$, the expected NPV is $\$ 3,400,000$ and the standard deviation is $\$ 405,000$. In the first generation, the best well (in either case) had an expected NPV of less than $\$ 2,900,000$. As would be expected, both the expected NPV and the standard deviation are lower in the risk averse case. This example illustrates the ability of the GA procedure to identify optimal nonconventional wells under geological uncertainty. 


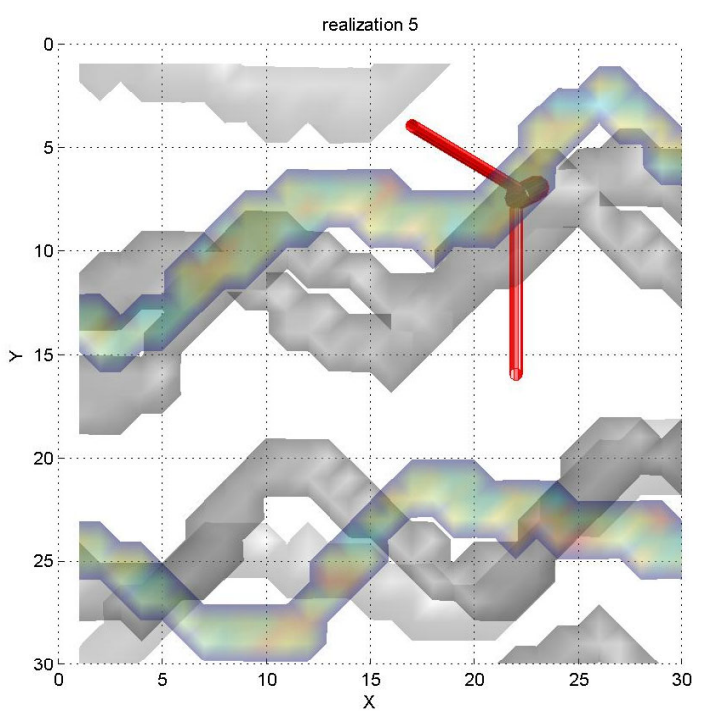

(a)

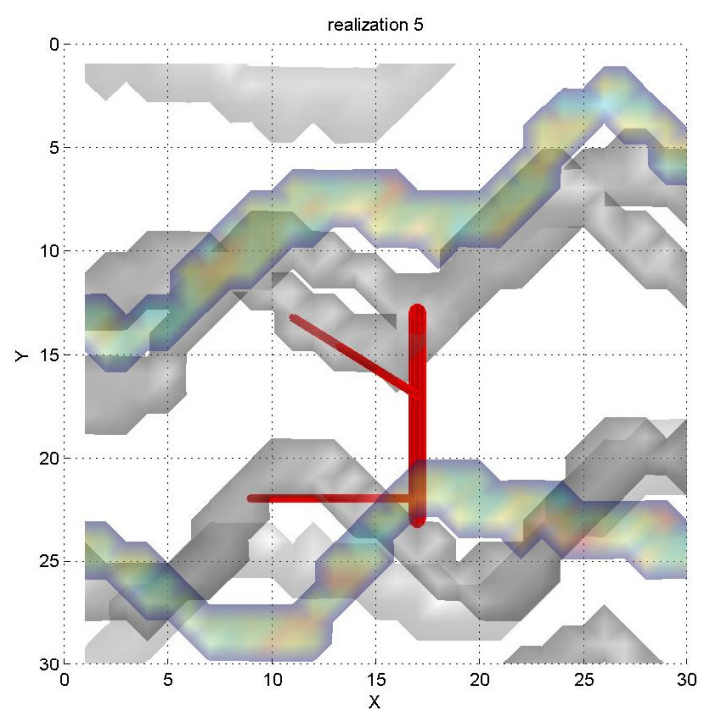

(b)

Figure 7-5: Permeability realization and optimal wells for (a) risk neutral and (b) risk averse cases

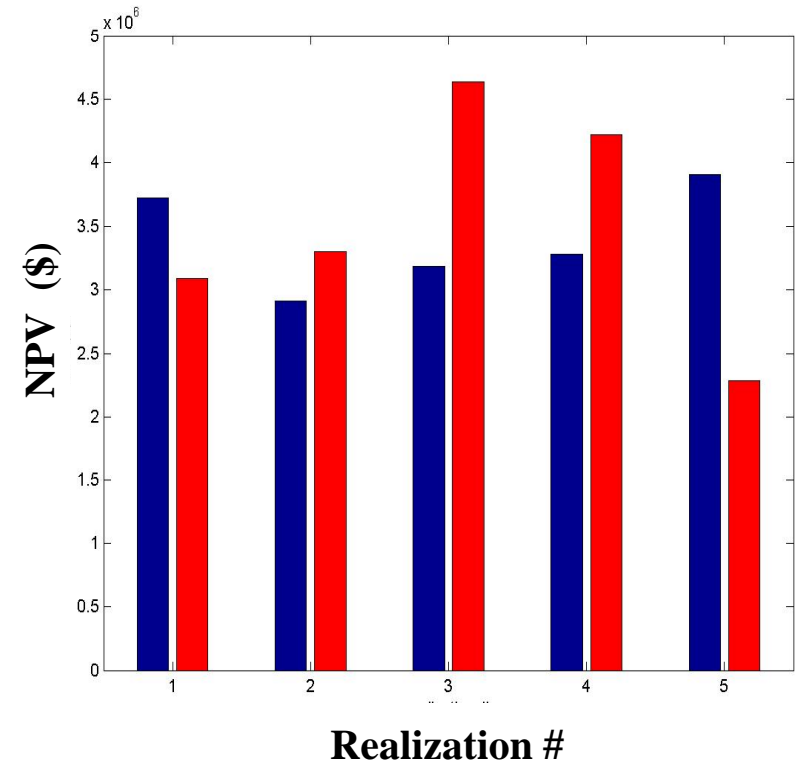

Figure 7-6: NPV with optimal well for each realization for risk neutral (red) and risk averse (blue) cases 


\subsection{Linkage of Smart Well Control and History Matching}

\subsubsection{Optimization Procedure}

The smart well optimization applied here utilizes a conjugate gradient procedure as described earlier in Yeten et al. (2004) and Aitokhuehi (2004). The optimization routine exists outside of the reservoir simulator and uses the simulator for function evaluations. The advantage of this approach is that a commercial simulator (Schlumberger, 2001) with a sophisticated well model (Holmes et al., 1998) can be applied. This procedure is, however, considerably more time consuming than adjoint solution techniques (such as that of Brouwer and Jansen, 2002), which require many fewer simulation runs. Adjoint techniques, by contrast, require a close link (essentially at the level of source code) with the simulator, which our numerical gradient approach avoids. For optimizations involving relatively few downhole valves and infrequent updating of their settings, the numerical gradient approach is viable. For more general situations, adjoint techniques are likely to be the preferable option.

The valve settings are updated at specified times during the course of the simulation. We investigated various approaches for performing this optimization, including optimizing over periods beyond the time of the next valve update. The idea behind this approach is to avoid valve settings that are "optimal" over a particular time period but lead to detrimental effects at later times. We did not, however, observe much sensitivity to the optimization time period in a number of example cases (see Aitokhuehi, 2004). In the simulations below, we nonetheless apply the approach of Yeten et al. (2004), in which the valve settings at each optimization step are determined by optimizing over the entire (remaining) simulation time. In some cases we determine valve settings that are optimal (in an average sense) over multiple realizations, using an approach along the lines of that used in the GA calculations (i.e., Eqs. 7-5 and 7-6).

\subsubsection{History-Matching Procedure}

The technique used here is the probability perturbation approach developed by Caers (2003). This method is based on multiple point (mp) geostatistics in which the geological model is characterized via a "training image." Multiple point geostatistics include two point geostatistical (variogram-based) models as special cases. The history matching procedure seeks to minimize the sum of the squares of the differences between the observed and predicted production data. The algorithm entails the gradual modification of the initial $\mathrm{mp}$ permeability field until the 
production data are honored. This is accomplished through the use of a non-stationary Markov chain procedure that is parameterized via a single transition variable. This variable is determined at each iteration of the history matching procedure through a one-dimensional optimization.

For purposes of the history match, we assume that each of the downhole sensors provides individual phase flow rates. For a multilateral well in which each branch is instrumented, this means that the pressure and phase flow rates in each lateral are assumed to be known. In current applications, downhole sensors do not yet provide this information directly (though flow rates can be estimated from temperature and pressure measurements), but it is reasonable to assume that future sensors will provide such data more directly and with greater degrees of accuracy.

\subsubsection{Overall Optimization and History Matching Procedure}

We identify one particular realization as the reference model. This model is simulated in order to generate the "production data" used for the history matching. This simulation uses the historical valve settings for previous time periods and the settings as determined from the most recent history-matched model to generate new production data. The history-matched model used for the determination of the optimal valve settings uses only the production data generated from the reference model. The history-matched models are, however, generated from the same training image as the reference model, so there is a general geological correspondence between them. Because the history-matched model is updated in time using production data, as time proceeds this model can be expected to more closely resemble the actual (reference) model and the optimized valve settings should provide improved performance. For full details on the combined procedure, see Aitokhuehi (2004).

\subsection{Results Using Optimization and History Matching}

We now present results for two different channelized systems, referred to as fluva and fluvb. The two systems represent unconditional realizations generated from different training images using the snesim software (Strebelle, 2000). Independent and unconditional population of the permeability and porosity within each facies was performed with sgsim (Deutsch and Journel, 1998). The channel sand was of average permeability 436 md (average porosity 0.24) while the mudstone was of average permeability $10 \mathrm{md}$ (average porosity 0.07 ). The physical size of the 
system was $4000 \mathrm{ft} \times 4000 \mathrm{ft} \times 100 \mathrm{ft}$ (the upper $50 \mathrm{ft}$ represent a gas cap; an analytical aquifer acts at the bottom edge of the model) and the simulation models were of dimension $20 \times 20 \times 6$. The mobility ratio was slightly less than one.

A quadlateral well located $20 \mathrm{ft}$ above the oil-water contact was introduced into the model. The mainbore was of a total length of $3200 \mathrm{ft}$ and each of the laterals was approximately $2200 \mathrm{ft}$. Only the laterals (not the mainbore) were opened to production. The well and permeability field are shown in Fig. 7-7. Constant total fluid rate control was specified for the well, subject to a minimum bottomhole pressure of 1,500 psi. Initial production was specified at a total liquid rate of $10 \mathrm{MSTB} / \mathrm{d}$. The simulation proceeded for 800 days and the valve settings were updated every 200 days (i.e., the simulation period was divided into four optimization and history matching steps). The entire well was shut-in if the producing water cut exceeded $80 \%$ or if the oil rate fell below the economic limit of $200 \mathrm{STB} / \mathrm{d}$.

We assess the performance of the overall method relative to two reference cases. These are (1) simulation using the actual (known) geology but with no valves (uninstrumented base case) and (2) simulation using the actual geology with optimized valves. We expect that the results from our method (optimized valves but unknown geology) will fall between these two results.

Simulation results for the fluva model are shown in Fig. 7-8. Plotted here are (a) the cumulative oil production and (b) water cut for the uninstrumented base case (blue curve), for the case of known geology and optimized valves (green curve), and for the case using our overall procedure (optimized valves and history matching, indicated by the red curve). We first consider the improvement attained using optimized valves for the case of known geology (blue and green curves). It is apparent that the valves lead to significantly improved performance - specifically, an increase in cumulative oil of about $40 \%$ over the uninstrumented base case. This improvement is directly related to a reduction in water cut, as the model with no valves reaches the water cut limit at about 420 days, while the optimized valve case does not reach this limit over the time period considered. This is evident from the water cut behavior (Fig. 7-8b). 


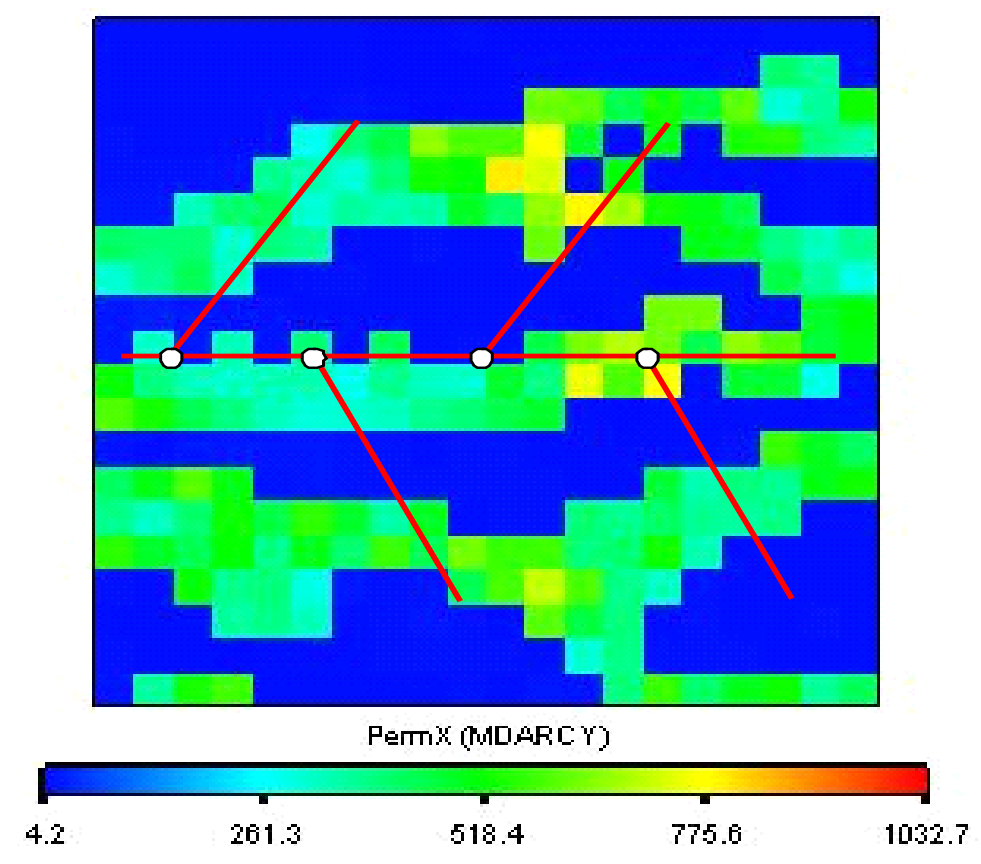

Figure 7-7: Permeability map of fluva channel reservoir with quadlateral well
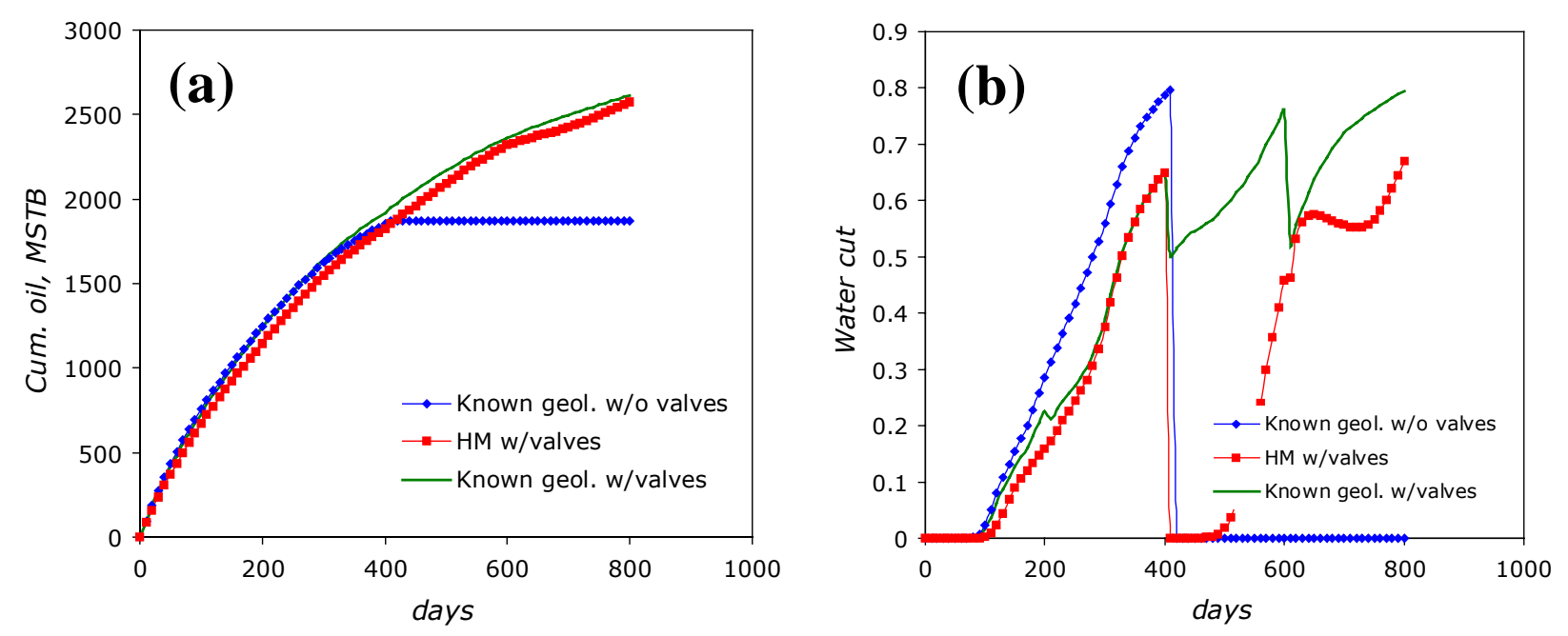

Figure 7-8: (a) cumulative oil and (b) water cut for the overall procedure and known geology cases 
We next consider the results using the valve optimization plus history matching (HM) procedure. As is apparent in Fig. 7-8a, the procedure leads to results very close to those obtained when the geology is known. Specifically, the technique provides an increase in cumulative oil of about $38 \%$ over the uninstrumented base case, which is nearly as much as the $40 \%$ improvement achieved for the case of known geology. This demonstrates the potential gains that can be achieved using this methodology. It is interesting to note, however, that the water cut from the optimized case with unknown geology is quite different than that for the case of known geology, indicating that the optimizations were achieved via different paths.

The second case simulated is referred to as fluvb. For this case, the channel sand was of higher average permeability than for fluva $(1660 \mathrm{md})$, though the model and well were otherwise the same as in the previous example. Following the same procedure as above, we observed an increase of $28 \%$ in cumulative oil production (relative to the uninstrumented base case) when optimized valves were used with known geology. However, when we applied our overall history matching plus optimization procedure to this case, the results were disappointing; i.e., we did not achieve results very close to the optimized case with known geology, as we did previously. We then tried several different initial geological models and applied the procedure using these models one at a time. We observed on average about a $12 \%$ improvement in cumulative oil recovery relative to the uninstrumented base case, much less than the $28 \%$ improvement observed when the geology was known. This $12 \%$ improvement is about the same as was achieved using optimization without history matching; i.e., by simply optimizing on the initial realizations.

The problem here appears to derive from the inherent nonuniqueness (and thus uncertainty) in the history-matched model. The inaccuracy in any of the individual history-matched models renders them incapable of providing optimization results comparable to those achieved for known geology. We address this issue by optimizing the valve settings over multiple historymatched models. This is accomplished by determining settings that are optimal, in an average sense, over sets of three or five models. In order to present the results using this procedure more concisely, we introduce a parameter $(\Delta N)$ that quantifies the improvement in cumulative oil recovery $\left(N_{p}\right)$ relative to that attained for the case of known geology: 


$$
\Delta N=\frac{N_{p_{\text {target model, w/valve }}}-N_{p_{\text {known geology, no valve }}}}{N_{p_{\text {known geology, w/valve }}}-N_{p_{\text {known geology, no valve }}}}
$$

If $\Delta N=0$, the cumulative oil recovered from the target model is the same as that of the uninstrumented base case with known geology. On the other hand, if $\Delta N=1$ (presumably the maximum attainable), the cumulative oil recovered from the target model is the same as that of the optimized case with known geology. Higher values of $\Delta N$ are of course desirable; for reservoir fluva considered above, we obtained $\Delta N \approx 0.94$.

The results achieved by optimizing over multiple realizations are displayed in Table 7-1. From the table, we see that $\Delta N \approx 0.4$ if we optimize over one history-matched (HM) model or if we optimize over multiple models that have not been history-matched. Optimizing over three or five history-matched models, however, leads to significant improvement in recovery; i.e., $\Delta N \approx$ 0.85 . This clearly demonstrates the benefit of minimizing the error inherent in any single historymatched model by considering multiple such models.

Also reported in the table are the standard deviations in $\Delta N$. These values were determined by simulating multiple (five or more) groups of one, three, and five models (both with and without history matching). Note that we do not report standard deviations for the case of five history-matched models because only two such groups were considered, due to the computational costs of these runs. The standard deviations in $\Delta N$ with history-matching demonstrate that the use of multiple history-matched models also leads to lower variations; i.e., less sensitivity to the particular set of initial realizations used for the history matching.

We also considered optimizing over multiple history-matched models for the fluva case considered above. Here, using individual history-matched models, $\Delta N=0.90 \pm 0.18$. Using three models, this improved to $\Delta N=0.94 \pm 0.04$; with five models $\Delta N=0.93$ (again, we did not perform enough runs for this case to report the standard deviation). These results indicate that the use of more than one model acts to improve $\Delta N$ and decrease the standard deviation. However, in some cases, such as this one, a single history-matched model may suffice for purposes of the optimization. 
Table 7-1: Impact of optimizing over multiple history-matched models (fluvb)

\begin{tabular}{cll}
\hline Number of & & \\
HM models & & \\
& & \\
\hline 1 & 0.393 without $\mathrm{HM}$ & $\mathrm{HM}$ \\
3 & $0.417 \pm 0.372$ & $0.438 \pm 0.273$ \\
5 & $0.358 \pm 0.410$ & $0.852 \pm 0.165$ \\
\hline
\end{tabular}

Finally, we applied the overall valve optimization - history matching procedure to cases in which the permeability field was conditioned to well data. We assumed that the facies type was known along the mainbore and along all of the laterals (as could be achieved using LWD). Results for $\Delta N$ (using a single model) are shown in Table 7-2. These results represent averages over many (eight or more) models used one at a time. With conditioning and history matching, the use of a single realization provides improved results relative to the case with no history matching or conditioning for the fluvb model ( $\Delta N=0.645$ compared to 0.393$)$. Multiple historymatched models were also considered for fluvb. In this case, using three history-matched models ( six such sets were considered), $\Delta N=0.83 \pm 0.10$, which is quite similar to what we obtained using history matching without conditioning (Table 7-1). It is possible that conditioning does not have more of an impact on $\Delta N$ in some cases because there is a degree of redundancy in the facies and production data (so the benefit of conditioning is not that great). This issue requires further investigation.

Table 7-2: Impact of conditioning on optimization results

\begin{tabular}{cccc}
\hline Model & $\begin{array}{r}\Delta N \text { w/o HM, } \\
\text { w/o cond }\end{array}$ & $\begin{array}{c}\Delta N \text { w/o HM, } \\
\text { w/ cond }\end{array}$ & $\begin{array}{c}\Delta N \text { w/HM, } \\
\text { w/cond }\end{array}$ \\
\hline fluva & $0.519 \pm 0.255$ & $0.581 \pm 0.174$ & $0.881 \pm 0.062$ \\
fluvb & $0.393 \pm 0.508$ & $0.543 \pm 0.270$ & $0.645 \pm 0.173$ \\
\hline
\end{tabular}


It should be noted that the method presented in this paper is very computationally intensive. For the cases considered here (four valve updates and four history matches), the valve optimizations required a total of about 100 simulations for the gradient calculations. The history matching algorithm required many more simulations, as many as 200 runs each time the model was updated. These computational requirements are even greater when multiple realizations are considered. It should be noted, however, that we could have used many fewer history-matching runs (40-50) if we used a less stringent convergence tolerance. It is likely that this would have a relatively minor impact on the results, particularly in the case of optimizing over multiple history-matched models. Nonetheless, it would be desirable to accelerate the overall procedure to enable frequent model updates and valve optimizations in practice.

\subsection{Summary}

This work can be summarized as follows:

- A genetic algorithm for determining the optimal nonconventional well type and location was developed. The technique allows the well type (e.g., mono bore, trilateral) to evolve over the optimization.

- The genetic algorithm was applied to cases involving both deterministic and uncertain geological descriptions. Significant improvement in the objective function was observed over the course of the optimization.

- A combined history matching - smart well optimization procedure was implemented. The method uses numerical gradients in conjunction with a commercial simulator for the optimization and a probability perturbation approach based on multiple point geostatistics for history matching.

- Using the combined technique, the resulting production was nearly as much as that achieved using optimized valves with known geology, indicating the potential benefits of the overall approach.

- The computational requirements of the techniques described here are very substantial. In the future, we plan to develop significantly more efficient methods that are better suited for practical use. 


\section{Acknowledgments}

We thank Prof. J. Caers for providing us with history matching algorithms and for his assistance in their use. V. Artus thanks Institut Français du Pétrole for partial funding.

\subsection{References}

Aitokhuehi, I.: "Real-time Optimization of Smart Wells," MS thesis, Stanford University (2004).

Bittencourt, A.C., Horne, R.N.: "Reservoir Development and Design Optimization," paper SPE 38895 presented at the SPE Annual Technical Conference and Exhibition, San Antonio, Oct. 58, 1997.

Brouwer, D.R., Jansen, J.D.: "Dynamic Optimization of Water Flooding with Smart Wells Using Optimal Control Theory," paper SPE 78278 presented at the SPE European Petroleum Conference, Aberdeen, UK, Oct. 29-31, 2002.

Caers, J.: "History Matching Under Training-Image-Based Geological Model Constraints," SPEJ, 8, 218-226 (Sept. 2003).

Centilmen, A., Ertekin, T., Grader, A.S.: “Applications of Neural Networks in Multiwell Field Development," paper SPE 56433 presented at the SPE Annual Technical Conference and Exhibition, Houston, Oct. 3-6, 1999.

Deutsch, C.V., Journel, A.G.: GSLIB User's Manual, $2^{\text {nd }}$ edition, Oxford University Press, New York (1998).

Güyagüler, B., Horne, R.N.: "Uncertainty Assessment of Well Placement Optimization," paper SPE 71625 presented at the SPE Annual Technical Conference and Exhibition, New Orleans, Sept. 30 - Oct. 3, 2001.

Güyagüler, B., Horne, R.N., Rogers, L., Rosenzweig, J.J.: "Optimization of Well Placement in a Gulf of Mexico Waterflooding Project,” SPEREE, 229 (June 2002).

Holmes, J.A., Barkve, T., Lund, O.: "Application of a Multisegment Well Model to Simulate Flow in Advanced Wells," paper SPE 50646 presented at the SPE European Petroleum Conference, The Hague, Netherlands, Oct. 20-22, 1998.

Montes, G., Bartolome, P., Udias, A.L.: "The Use of Genetic Algorithms in Well Placement Optimization," paper SPE 69439 presented at the SPE Latin American and Caribbean Petroleum Engineering Conference, Buenos Aires, March 25-28, 2001.

Reed, R.R., Marks II, R.J.: Neural Smithing, Supervised Learning in Feedforward Artificial Neural Networks, MIT Press, Cambridge, MA (1999). 
Santellani, G., Hansen, B., Herring, T.: "'Survival of the Fittest': An Optimized Well Location Algorithm for Reservoir Simulation," paper SPE 39754 presented at the SPE Asia Pacific Conference on Integrated Modelling for Asset Management, Kuala Lumpur, March 23-24, 1998.

Schlumberger GeoQuest: ECLIPSE Technical Description Manual, 2001.

Seifert, D., Lewis, J.J.M., Hern, C.Y., Steel, N.C.T.: "Well Placement Optimisation and Risking Using 3D Stochastic Reservoir Modelling Techniques," paper SPE 35520 presented at the SPE European 3D Reservoir Modeling Conference, Stavanger, Norway, April 16-17, 1996.

Sinha, S., Vega, L., Kumar, R., Jalali, Y.: "Flow Equilibration Toward Horizontal Wells Using Downhole Valves - A Single Well Study on Dual Drive," paper SPE 68635 presented at the Asia Pacific Oil and Gas Conference and Exhibition, Jakarta, Indonesia, April 17-19, 2001.

Strebelle, S.: "Sequential Simulation Drawing Structure from Training Images," PhD thesis, Stanford University (2000).

Sudaryanto, B., Yortsos, Y.C.: “Optimization of Fluid Front Dynamics in Porous Media Using Rate Control. I. Equal Mobility Fluids,” Physics of Fluids, 12, 1656-1670 (2000).

Yeten, B.: "Optimum Deployment of Nonconventional Wells," PhD thesis, Stanford University (2003).

Yeten, B., Brouwer, D.R., Durlofsky, L.J., Aziz, K.: "Decision Analysis under Uncertainty for Smart Well Deployment," to appear in J. Pet. Sci. \& Eng. (2004).

Yeten, B., Durlofsky, L.J., Aziz, K.: "Optimization of Nonconventional Well Type, Location, and Trajectory," SPEJ, 8, 200-210 (Sept. 2003).

Yeten, B., Jalali, Y.: "Effectiveness of Intelligent Completions in a Multi-well Development Context," paper SPE 68077 presented at the SPE Middle East Oil Show, Bahrain, March 17-20, 2001. 